\title{
Quantity and Quality of Stormwater Runoff from Western Daytona Beach, Florida, and Adjacent Areas
}

By G.F. Taylor

U.S. GEOLOGICAL SURVEY

Water-Resources Investigations Report 90-4002

Prepared in cooperation with the CITY OF DAYTONA BEACH

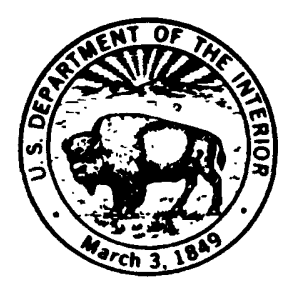




\section{U.S. DEPARTMENT OF THE INTERIOR MANUEL LUJAN, JR., Secretary \\ U.S. GEOLOGICAL SURVEY \\ Dallas L. Peck, Director}

For additional information write to:

District Chief U.S. Geological Survey Suite 3015 227 North Bronough Street Tallahassee, Florida 32301
Copies of this report can be purchased from:

U.S. Geological Survey Books and Open-File Reports Federal Center, Building 810 Box 25425

Denver, Colorado 80225 


\title{
CONTENTS
}

\author{
Abstract 1 \\ Introduction 1 \\ Purpose and scope 1 \\ Description of the study area 3 \\ Tomoka River basin 3 \\ B-19 Canal basin 3 \\ Previous investigations 3 \\ Methods of data collection 3 \\ Rainfall 6 \\ Stage and discharge 6 \\ Water-quality 6 \\ Quantity of stormwater runoff 6 \\ Quality of stormwater runoff 26 \\ Loads for selected constituents 28 \\ Summary 29 \\ Selected references $\mathbf{3 0}$ \\ Appendix I-Daily rainfall 33 \\ Appendix II-Daily stage 47 \\ Appendix III-Daily discharge 51 \\ Appendix IV-Quality of water analyses 67 \\ Appendix V-Daily water temperature 73 \\ Appendix VI-Daily specific conductance 79 \\ Appendix VII-Daily dissolved oxygen 85
}

\section{FIGURES}

1-2. Maps showing:

1. Study area 2

2. Location of data-collection sites and surface-water station drainage-basin boundaries 4

3-11. Hydrographs showing daily discharges from October 1982 through September 1985 for:

3. Site 1, Tomoka River near Holly Hill 7

4. Site 2, Eleventh Street Canal near Holly Hill 7

5. Site 3, Williamson Boulevard Ditch at Daytona Beach 8

6. Site 4, Wally Hoffmeyer Canal at Daytona Beach 8

7. Site 5, Thayer Canal near Daytona Beach 9

8. Site 6, Bayless Boulevard Canal at Daytona Beach 9

9. Site 7, Bellevue Canal at Daytona Beach 10

10. Site 11, B-19 Canal at Willow Run Boulevard at Port Orange 10

11. Site 12, B-19 Canal at Dunlawton Avenue at Port Orange 11

12-20. Hydrographs for selected storms at:

12. Site 1, Tomoka River near Holly Hill $\mathbf{1 2}$

13. Site 2, Eleventh Street Canal near Holly Hill 13

14. Site 3, Williamson Boulevard Ditch at Daytona Beach 14

15. Site 4, Wally Hoffmeyer Canal at Daytona Beach 15

16. Site 5, Thayer Canal near Daytona Beach 16

17. Site 6, Bayless Boulevard Canal at Daytona Beach 17

18. Site 7, Bellevue Canal at Daytona Beach 18 
19. Site 11, B-19 Canal at Willow Run Boulevard at Port Orange 19

20. Site 12, B-19 Canal at Dunlawton Avenue at Port Orange 20

21-29. Graphs showing rainfall-runoff plots for:

21. Site 1, Tomoka River near Holly Hill 22

22. Site 2, Eleventh Street Canal near Holly Hill 22

23. Site 3, Williamson Boulevard Ditch at Daytona Beach 23

24. Site 4, Wally Hoffmeyer Canal at Daytona Beach 23

25. Site 5, Thayer Canal near Daytona Beach 24

26. Site 6, Bayless Boulevard Canal at Daytona Beach 24

27. Site 7, Bellevue Canal at Daytona Beach 25

28. Site 11, B-19 Canal at Willow Run Boulevard at Port Orange 25

29. Site 12, B-19 Canal at Dunlawton Avenue at Port Orange 26

\section{TABLES}

1. Hydrologic data-collection sites 5

2. Summary of Thiessen-method rainfall and resulting runoff for selected storms 21

3. Summary of selected constituent limits and data from samples collected at surface-water sites 27

4. Phosphorus, nitrogen, and dissolved-solids loads for selected storms in the Tomoka River and B-19 Canal basins 29 


\section{CONVERSION FACTORS, ABBREVIATIONS, AND DEFINITIONS}

For use of those readers who may prefer to use metric (International System) units, conversion factors for the terms used in this report are listed below:

\begin{tabular}{|c|c|c|}
\hline Multiply inch-pound unit & By & To obtain metric unit \\
\hline \multicolumn{3}{|l|}{ Length } \\
\hline inch (in.) & 2.54 & centimeter $(\mathrm{cm})$ \\
\hline foot (ft) & 0.3048 & meter (m) \\
\hline mile (mi) & 1.609 & kilometer (km) \\
\hline \multicolumn{3}{|l|}{ Area } \\
\hline square mile $\left(\mathrm{mi}^{2}\right)$ & 2.590 & square kilometer $\left(\mathrm{km}^{2}\right)$ \\
\hline \multicolumn{3}{|l|}{ Weight } \\
\hline ton (ton) & 908 & kilogram (kg) \\
\hline pound (lb) & 0.454 & kilogram (kg) \\
\hline pound (lb) & 454,000 & milligram (mg) \\
\hline pound per square mile $\left(\mathrm{lb} / \mathrm{mi}^{2}\right)$ & 0.454 & kilogram per square mile $\left(\mathrm{kg} / \mathrm{mi}^{2}\right)$ \\
\hline \multicolumn{3}{|l|}{ Volume } \\
\hline cubic foot $\left(\mathrm{ft}^{3}\right)$ & 0.02832 & cubic meter $\left(\mathrm{m}^{3}\right)$ \\
\hline cubic foot $\left(\mathrm{ft}^{3}\right)$ & 28.32 & liter (L) \\
\hline cubic foot $\left(\mathrm{ft}^{3}\right)$ & 28,320 & milliliter (mL) \\
\hline \multicolumn{3}{|l|}{ Fow } \\
\hline pound per day (lb/d) & 0.454 & kilogram per day $(\mathrm{kg} / \mathrm{d})$ \\
\hline cubic foot per second $\left(\mathrm{ft}^{3} / \mathrm{s}\right)$ & 0.02832 & cubic meter per second $\left(\mathrm{m}^{3} / \mathrm{s}\right)$ \\
\hline
\end{tabular}

\section{Additional abbreviations}

$$
\begin{aligned}
\mu \mathrm{g} / \mathrm{kg} & =\text { microgram per kilogram } \\
\mathrm{mg} / \mathrm{L} & =\text { milligram per liter } \\
\mu \mathrm{g} / \mathrm{L} & =\text { mictogram per liter }
\end{aligned}
$$

$\mu \mathrm{S} / \mathrm{cm}$ at $25^{\circ} \mathrm{C}=$ microsiemen per centimeter at 25 degrees Celsius

Temperature in degrees Fahrenheit $\left({ }^{\circ} \mathrm{F}\right)$ can be converted to degrees Celsius $\left({ }^{\circ} \mathrm{C}\right)$ as follows:

$$
{ }^{\circ} \mathrm{C}=5 / 9\left({ }^{\circ} \mathrm{F}-32\right)
$$

Sea level: In this report "sea level" refers to the National Geodetic Vertical Datum of 1929 (NGVD of 1929) - a geodetic datum derived from a general adjustment of the first-order level nets of both the United States and Canada; formerly called "Sea Level Datum of 1929." Altitude, as used in this report, refers to distance above or below sea level. 


\section{Contents}




\section{Quantity and Quality of Stormwater Runoff from Western Daytona Beach, Florida, and Adjacent Areas}

\author{
By G.F. Taylor
}

\section{ABSTRACT}

Runoff from western Daytona Beach and adjacent areas was monitored from November 1982 through September 1985. Quantity and quality of stormwaterrunoff data were collected at 12 surface-water sites and 8 rainfall sites, and then used in the hydrologic analysis of the upper Tomoka River basin and upper B-19 Canal basin.

Runoff, as a percentage of rainfall, for selected storm periods ranged from 1 to 77 percent for the Tomoka River basin, and 6 to 17 percent for the B-19 Canal basin. Few patterns were discernible from the rainfall-runoff plots.

Major nutrients were sampled at all sites; total phosphorus ranged from 0.01 to 0.52 milligram per liter, and total nitrogen concentrations ranged from 0.09 to 2.4 milligrams per liter. Dissolved-solids concentrations ranged from 46 to 458 milligrams per liter, and specificconductance values ranged from 49 to 865 microsiemens per centimeter. The conservative constituent chloride ranged from 3.4 to 57 milligrams per liter.

Eighty-seven percent of all samples analyzed for color exceeded the $\mathbf{4 5}$ platinum-cobalt units criterion suggested by the U.S. Environmental Protection Agency. Except for low $\mathrm{pH}$ values in two tributaries draining swampy land in the Tomoka River basin, $\mathrm{pH}$ values for both basins were within the recommended limits established by the Florida Department of Environmental Regulation. Dissolved-oxygen concentrations in the 4- to 6milligrams-per-liter range were common. Three insecticides (diazinon, heptachlor, and DDD) and one herbicide $(2,4-D)$ were detected in water or bottom sediments. Mercury concentrations in a total of five samples from both basins exceeded the 0.2 -microgramper-liter criterion for potable water supplies. Zinc concentrations did not exceed the recommended limits in any samples from the B-19 Canal basin but exceeded the recommended limits in 25 percent of the samples from the Tomoka River basin.

\section{INTRODUCTION}

Stormwater runoff is rainwater that leaves an area by means of surface drainage. The quantity and quality of stormwater runoff are influenced by rainfall and drainage-basin characteristics. Rainfall characteristics such as intensity, duration, and areal coverage influence mainly the quantity of stormwater runoff. Basin characteristics such as topography, geology, land use, vegetation, and soils influence both the quantity and quality of stormwater runoff.

Hydraulic and chemical properties of stormwater runoff are of primary concern in urban planning. The hydraulics of runoff control erosion, overbank flooding, and deposition of solids. In addition, the chemical quality of stormwater runoff affects the usefulness of the runoff water and the receiving waters.

Increased development within the western Daytona Beach area is anticipated in the near future. This development could possibly increase runoff volumes and constituent loads discharging to the Tomoka River and B-19 Canal. Information on the quantity and quality of stormwater runoff in developed and undeveloped areas in these basins is needed so that planners and managers can anticipate and minimize adverse effects of stormwater runoff on the receiving waters in the area. To provide the type of information needed by planners and managers, the U.S. Geological Survey in cooperation with the City of Daytona Beach, Fla., conducted a 3-year data collection and evaluation program for western Daytona Beach from 1982 to 1985.

The study area (fig. 1) is the upper parts of both the Tomoka River basin and the B-19 Canal basin, a tributary to Spruce Creek. This area includes western Daytona Beach and unincorporated adjacent areas west and south of the city, totaling about $71 \mathrm{mi}^{2}$ (square miles). Although most of the study area is undeveloped, the part within northwestern Daytona Beach north of U.S. Highway 92 is partly developed industrial-commercial. The study area south of State Road 400 within southwestern Daytona Beach is partly developed residential.

\section{Purpose and Scope}

This report presents results of the study to evaluate the quantity and quality of stormwater runoff from western Daytona Beach and adjacent areas, and summarizes the stormwater data collected. Rainfall, surface-water discharge, and water-quality data were collected from 


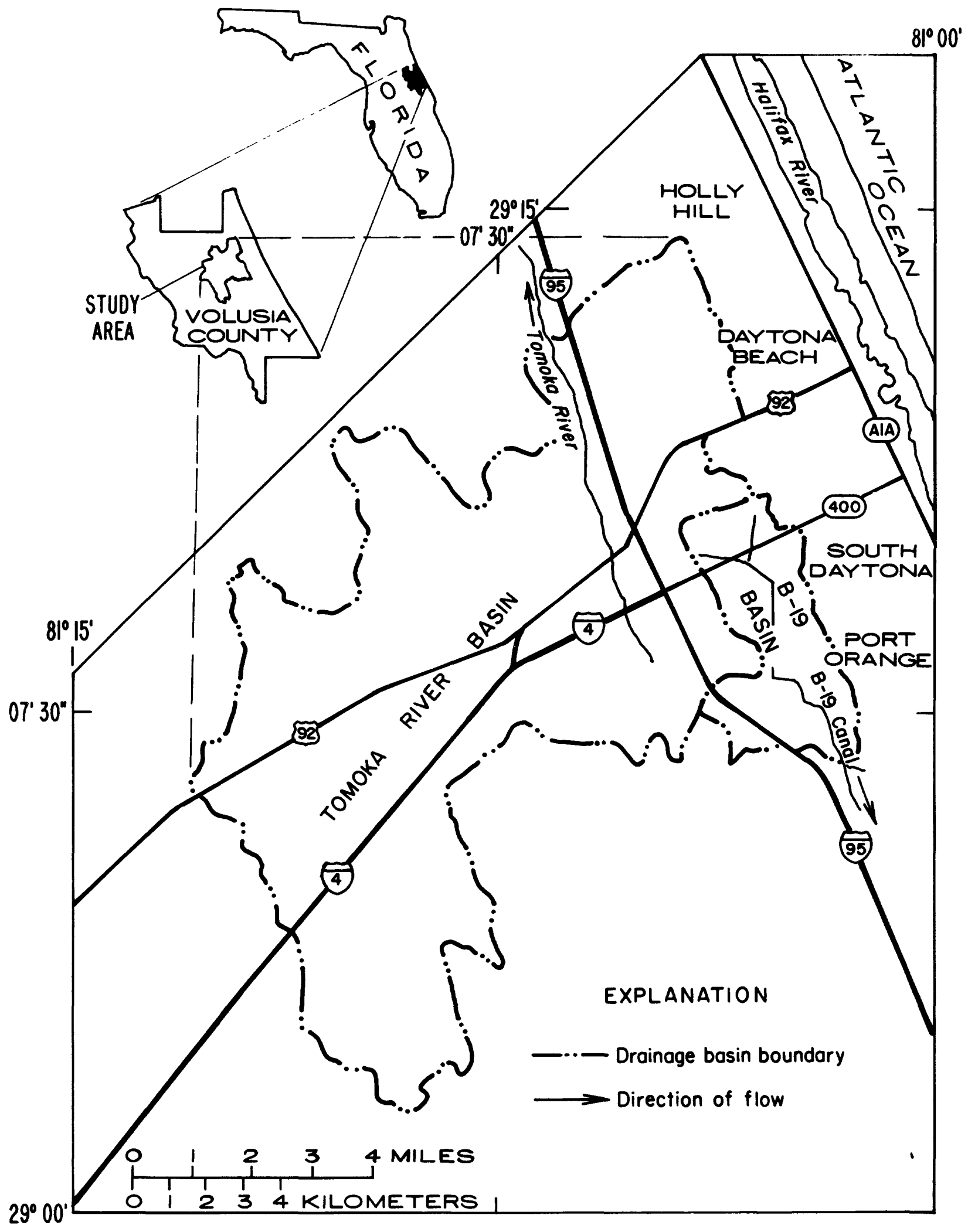

Figure 1. Study area. 
November 1982 through September 1985. The data are summarized in tables, hydrographs, and plots. The evaluation of stormwater runoff quality is based on a comparison of the results of chemical analyses of samples collected during this study with results from previous studies, and on criteria published by the Florida Department of Environmental Regulation (FDER) and the U.S. Environmental Protection Agency (USEPA).

\section{Description of the Study Area}

\section{Tomoka River Basin}

The upper Tomoka River basin, that part upstream from Eleventh Street, constitutes an area of about $63 \mathrm{mi}^{2}$. Approximately $9.5 \mathrm{mi}^{2}$ of this area is within the corporate limits of Daytona Beach. Most of the Upper Tomoka River basin is fairly flat and covered with pines, palmettos, dense underbrush, and marshes. The part of the basin within northwestern Daytona Beach is composed primarily of woods and partly developed industrial parks.

Because the water table throughout the basin is at or near land surface most of the time and the potentiometric surface is about 5 to 10 feet below the land surface (Rutledge, 1985), recharge is poor and large marshy areas exist. Most of the Tomoka River basin drains slowly because of the large storage capacity within the marshy areas and canals that have little bed slope.

Many ditches and canals cross subbasin boundaries and intersect other ditches and canals. This allows flow to move in either direction in the canals, depending upon hydraulic gradient. Intersecting channels having little bed slope are prevalent in the urban areas of northwest Daytona Beach and the Tiger Bay area along U.S. Highway 92 (figs. 1 and 2).

\section{B-19 Canal Basin}

The B-19 Canal basin, a tributary to Spruce Creek, has topography and vegetative cover similar to that in the Tomoka River basin. The part of the B-19 Canal basin within the study area constitutes an area of about $7.6 \mathrm{mi}^{2}$ above Dunlawton Avenue and south of the Daytona Beach Regional Airport (fig. 2). This upper part of the basin generally is low and flat with dense underbrush. The water table in this part of the basin is at or near land surface.

At site 11, the channel gradient changes dramatically with the downstream slope being about four times the upstream slope. This steep gradient contribules to greater stormwater velocities and deeper channels with greater flow capacities, thereby allowing stormwater to leave the area more quickly. In the upper reaches, constrictions at culvert entrances retard flow and cause water to go into temporary storage to such an extent that reversed flow was observed at site 10 on a number of occasions.

In the uppermost part of the basin, north of State Road 400, the basin is undeveloped, whereas south of State Road 400 , the basin is partly developed residential. The area along the east bank of B-19 Canal is most heavily developed. The west bank downstream from site 11 is mosily undeveloped.

\section{Previous Investigations}

A report by the Volusia County Planning Board (1978), entitled "Tomoka River Watershed Water Quality Study," summarizes the water quality in the Tomoka River watershed at that time. According to that report, the quality of the Tomoka River water was typical of a basin with large swampy areas and high in organic material. Generally, the water in the river was characterized as having moderate-to-low biodegradable organic content, high color, low dissolved-oxygen (DO) concentrations, high coliform-bacterial levels, and moderate-to-high nutrient concentrations. The high bacterial levels and nutrient concentrations could not be attributed to a single point source of contamination.

A series of statewide map reports by the U.S. Geological Survey (Kaufman, 1975a, b, c, and d) cover various aspects of water quality and hydrogeology for the study area. The information is general and accuracy is limited by the scale of the map used to show the information. A 1971 report by the U.S. Geological Survey gives some more specific information about water quality of the Tomoka River (Knochenmus and Beard, 1971).

\section{METHODS OF DATA COLLECTION}

Rainfall, stage and discharge of streams and canals, and water-quality data were collected from November 1982 through September 1985. Data-collection sites (fig. 2) include 12 surface-water sites, 7 on canals contributing to the Tomoka River, 2 on the Tomoka River, and 3 on the B-19 Canal. Rainfall-collection sites include six in the Tomoka River basin and two in the B-19 Canal basin. Table 1 lists the surface water and rainfall data-collection sites shown in fig. 2. Other information concerning these sites, such as type of data collected, equipment type, number of samples taken, contributing surface area, and geographical location are presented in table 1.

Equipment used to collect data included digital stage recorders, tipping-bucket rain gages, volumetric rainfall collectors, water-quality field monitors, automatic water samplers, and standard stream-gaging equipment. 


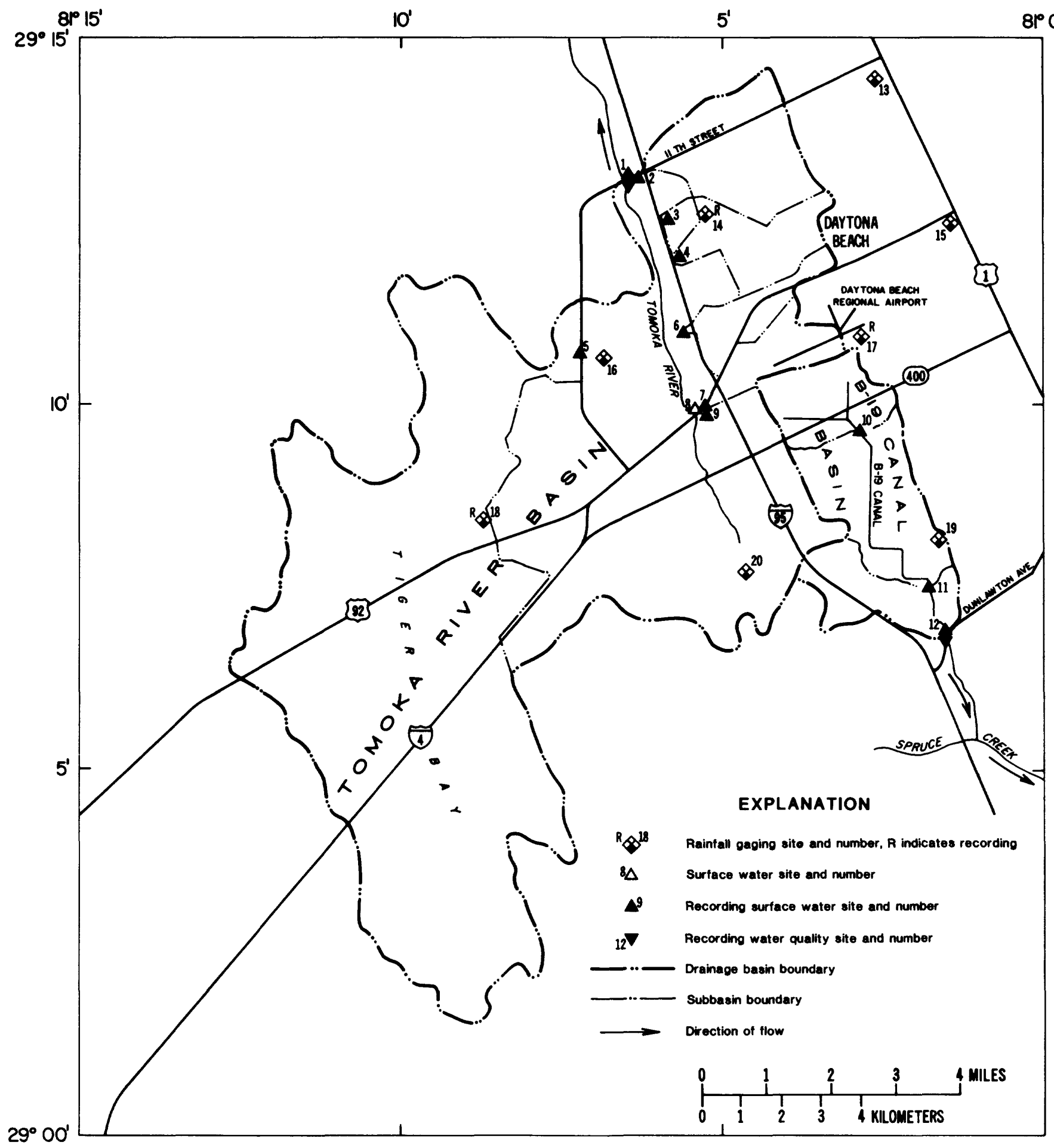

Figure 2. Location of data-collection sites and surface-water station drainage-basin boundaries. 
Table 1. Hydrologic data-collection sites

[Type of data: $S$, stage; $D$, discharge; $Q$, water quality; $P$, precipitation. Equipment type: $R$, recording; $N$, nonrecording.

Sampling period: $A$, aperiodic; $C$, continuous. Abbreviation: $\mathrm{mi}^{2}$, square miles; - indicates no data]

\begin{tabular}{|c|c|c|c|c|c|c|c|c|c|c|}
\hline $\begin{array}{l}\text { Site } \\
\text { No. }\end{array}$ & Location name & $\begin{array}{l}\text { Type } \\
\text { of } \\
\text { data }\end{array}$ & $\begin{array}{c}\text { Data } \\
\text { interval } \\
\text { (minutes) }\end{array}$ & $\begin{array}{l}\text { Equip- } \\
\text { ment } \\
\text { type }\end{array}$ & $\begin{array}{l}\text { Sam- } \\
\text { pling } \\
\text { period }\end{array}$ & $\begin{array}{c}\text { Number } \\
\text { of } \\
\text { samples }\end{array}$ & Latitude & Longitude & $\begin{array}{c}\text { Drainage } \\
\text { area } \\
\left(\mathrm{mi}^{2}\right)\end{array}$ & Station No. \\
\hline
\end{tabular}

Tomoka River basin

1 Tomoka River near Holly Hill

2 Eleventh Street Canal near Holly Hill

3 Williamson Boulevard Ditch at Daytona Beach

4 Wally Hoffmeyer Canal at Daytona Beach

5 Thayer Canal near Daytona Beach

6 Bayless Boulevard Canal at Daytona Beach

7 Bellevue Canal at Daytona Beach

8 U.S. Highway 92 Canal near Daytona Beach

9 Tomoka River near Daytona Beach

Surface-water sites

\section{B-19 Canal basin}

10 B-19 Canal near Daytona Beach

11 B-19 Canal at Willow Run Boulevard at Port Orange

12 B-19 Canal at Dunlawton Avenue at Port Orange

\section{Tomoka River basin}

13 Holly Hill

14 Florida Power and Light Substation

15 Daytona Marion Street Water Plant

16 Daytona Brennan Water Plant

17 NOAA Weather Station

18 Civil Defense Center

\section{B-19 Canal basin}

19 Port Orange

Water Plant

20 Volusia County Landfill

$\begin{array}{ccccc}\text { SQ } & 15 & \text { R } & \text { A } & 3 \\ \text { SDQ } & 15 & \text { R } & \text { A } & 5 \\ \text { SDQ } & 15 & \text { R } & \text { AC } & 5 \\ & & & & \\ & & & \end{array}$

290945

290730

290654

P $\quad \mathbf{1 , 4 4 0}$

P 60

P $\quad 1,440$

P $\quad 1,440$

$\begin{array}{ll}P & 60 \\ P & 60\end{array}$

$\mathbf{N}$
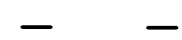

291433

0810247

-

291433081024700

R $\quad-\quad$ - 291237

0810514

0810123

0810657

0810253

0810808

290825

P $\quad 1,440$

P 1,440

290843

0810141

$-$

290843081014100

290744

0810428

290744081042800 
Water-quality data were collected at all surface-water sites. Some samples were collected by automatic sampling devices and some were collected manually.

\section{Rainfall}

Rainfall data were collected at eight sites, three recording and five nonrecording (fig. 2). One recordingrainfall site operated by the National Weather Service (site 17), and two operated by the U.S. Geological Survey (sites 14 and 18) collected rainfall data hourly. Daily totals from nonrecording sites (sites 13,15,16,19, and 20) were furnished by the City of Daytona Beach and other governmental agencies that collect rainfall data for their own use. Daily rainfall totals are listed in appendix I.

\section{Stage and Discharge}

Stage and discharge data were collected at sites 1 through 12 . Site 1 , Tomoka River near Holly Hill, which provided data at the downstream study boundary on the Tomoka River, has been in operation since October 1964. Additional surface-water stations were established at 11 sites; 8 in the Tomoka River basin and 3 in the B-19 Canal basin. Stage data were collected at 15-minute intervals to allow adequate observation of hydrograph response, except for site 8 where stage was measured aperiodically. Discharge was measured at various stages, and rating curves relating discharge to stage were established for 8 of the 11 new sites. Three of the new sites (sites 8,9 , and $10)$ were judged to have hydraulic conditions making them too difficult to rate. Daily mean stage data for sites 9 and 10 are given in appendix II. Daily mean discharge data are given in appendix III for all sites rated (sites 1-7, 11, and 12).

The delineated drainage areas for the surface-water sites are shown in figure 2. Field reconnaissance and the best available topographic maps were used to define drainage areas. However, drainage divides for sites 8 and 9 were indeterminate due to large marshy areas, flat slopes, and manmade interconnecting canals.

\section{Water Quality}

Water samples were collected at surface-water sites (sites 1 through 12) in order to describe the water quality within the two basins. Water-quality data were collected using manual sampling, automatic samplers, and continuousrecording water-quality monitors.
Samples were collected manually during storm and nonstorm conditions at all sites. These samples were analyzed for major ions, nutrients, and metals. Near the most downstream site in each basin (sites 2 and 12), one bottomsediment sample was collected for insecticide and herbicide analyses.

Some samples were collected automatically by sampling devices set to sample at a predetermined time interval after a selected stage was attained. These samplers were capable of collecting up to 28 discrete samples during a storm runoff event. The collected samples were then composited into one sample by discharge-weighting and analyzed for the same constituents and characteristics as individual samples. The results of the analyses for all samples are presented in appendix IV.

Water-quality monitors were used to collect long-term continuous temperature, conductance, and dissolved-oxygen data at sites 1 and 12, and short-term continuous temperature and conductance data at sites $2,3,4$, and 6 . These data are presented in appendixes V, VI, and VII.

\section{QUANTITY OF STORMWATER RUNOFF}

Discharge hydrographs for the 3-year study period were plotted for the nine sites having stage-discharge ratings to show the general long-term discharge patterns (figs. 3-11). Hydrographs for selected storms were also plotted to illustrate the runoff response more closely (figs. 12-20). As seen in these figures, hydrographs for the same site differ from storm to storm and response to the same storm differs from site to site. The hydrographs are affected by rainfall and basin characteristics. Rainfall characteristics include intensity, duration, and areal coverage of the rainfall. The basin characteristics include bed slope, channel geometry, vegetative cover, soil types, antecedent soil moisture, drainage area, and topographic relief.

In general, high-intensity, short-duration rainstorms produce quicker, higher peaks and have hydrographs that return to prestorm conditions faster. When one or more basin characteristic, such as dry soils, large contributing area, or vast swampy areas exist, the discharge hydrograph may show a reduced peak, a slower response, or longer-duration runoff. The effects of basin size can be seen when hydrographs for sites 3 and 2 are compared for the same storm. For example, the storm of September 1, 1985, (fig. 14), produced a peak at site 3 (Williamson Boulevard Ditch at Daytona Beach, drainage area $=0.3 \mathrm{mi}^{2}$ ) in 3 hours, with a return to base-flow condition in about 21 hours. 


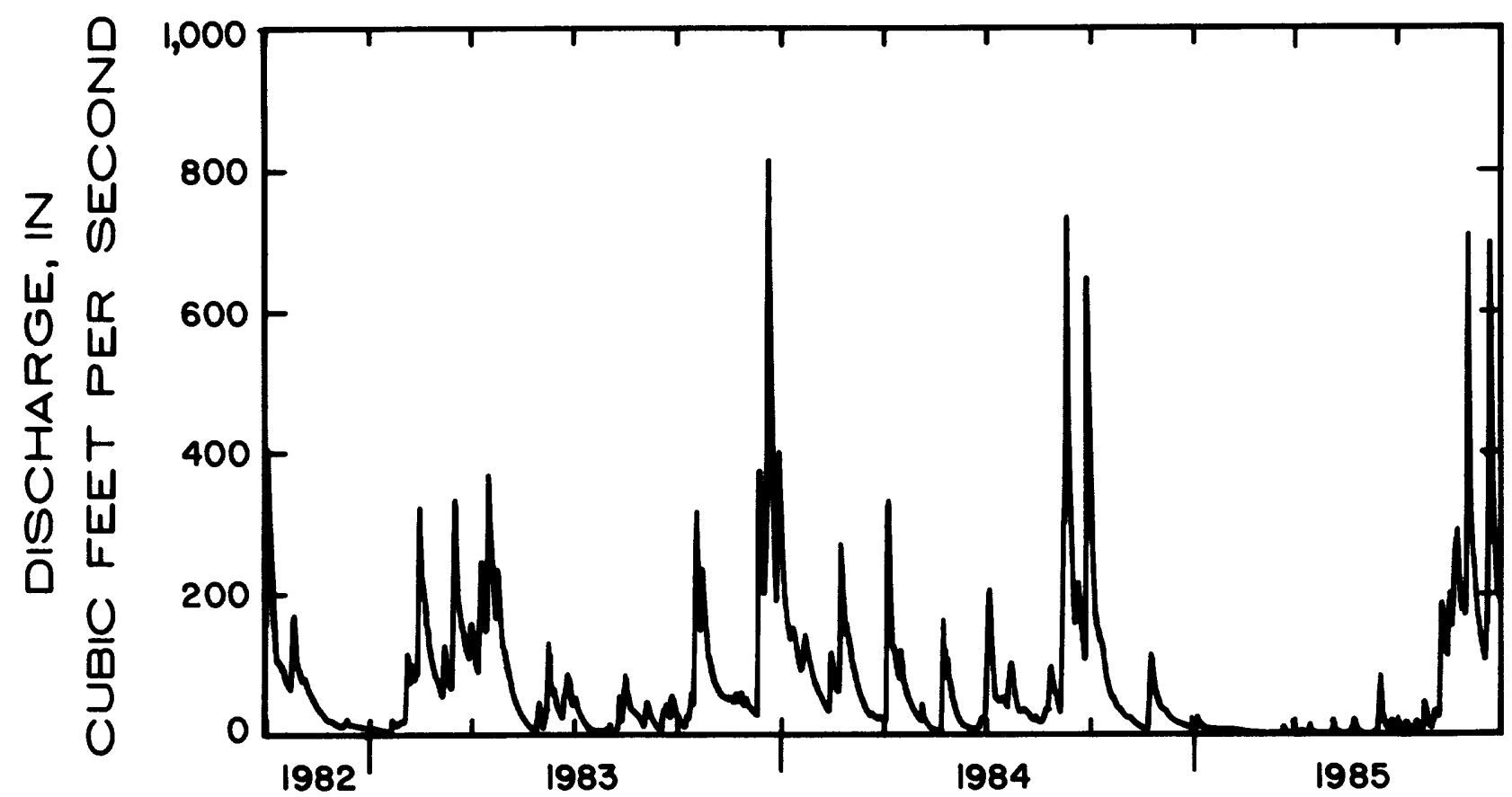

Figure 3. Daily discharges from October 1982 through September 1985 for site 1, Tomoka River near Holly Hill.

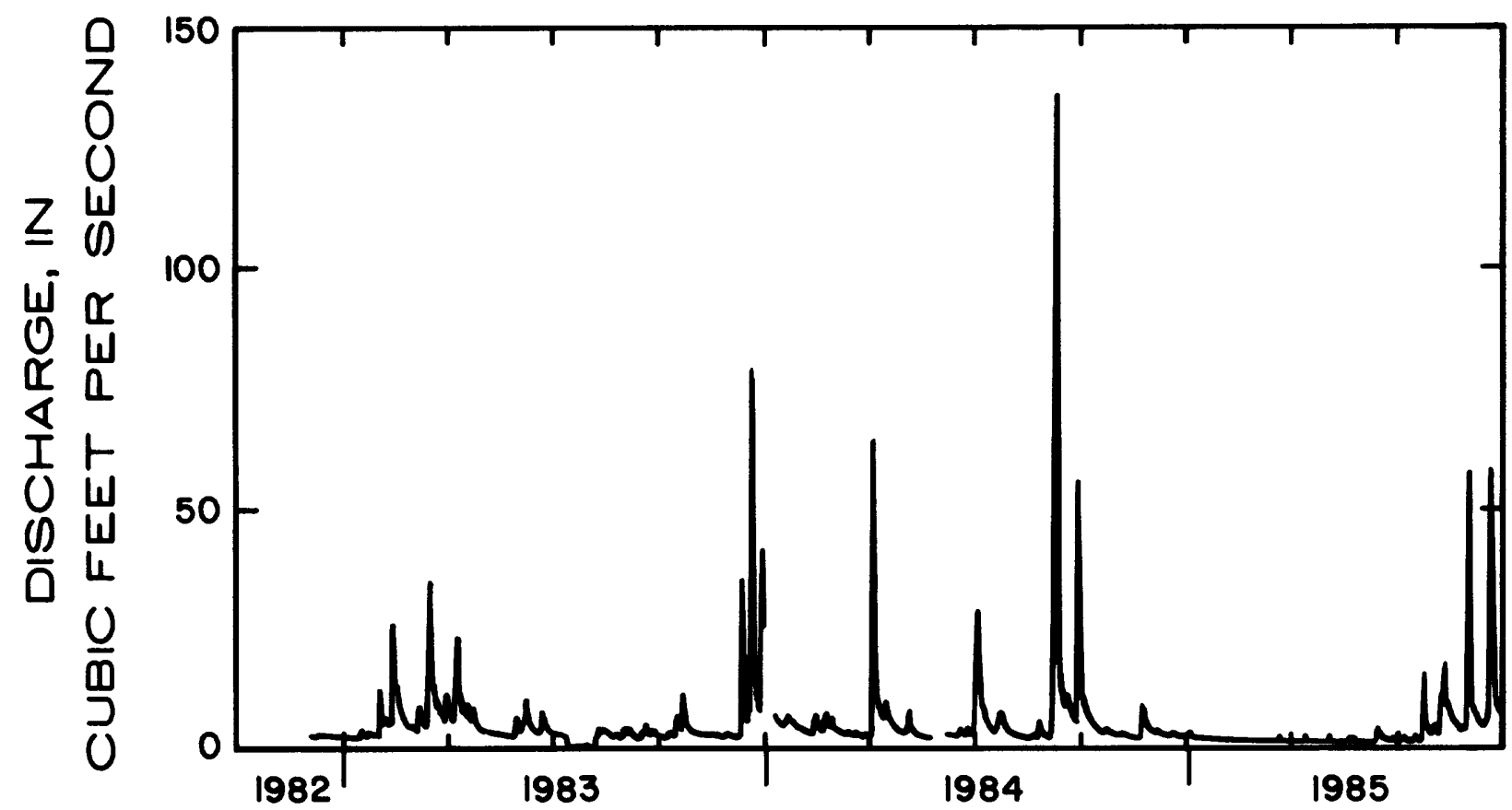

Figure 4. Daily discharges from October 1982 through September 1985 for site 2, Eleventh Street Canal near Holly Hill. 


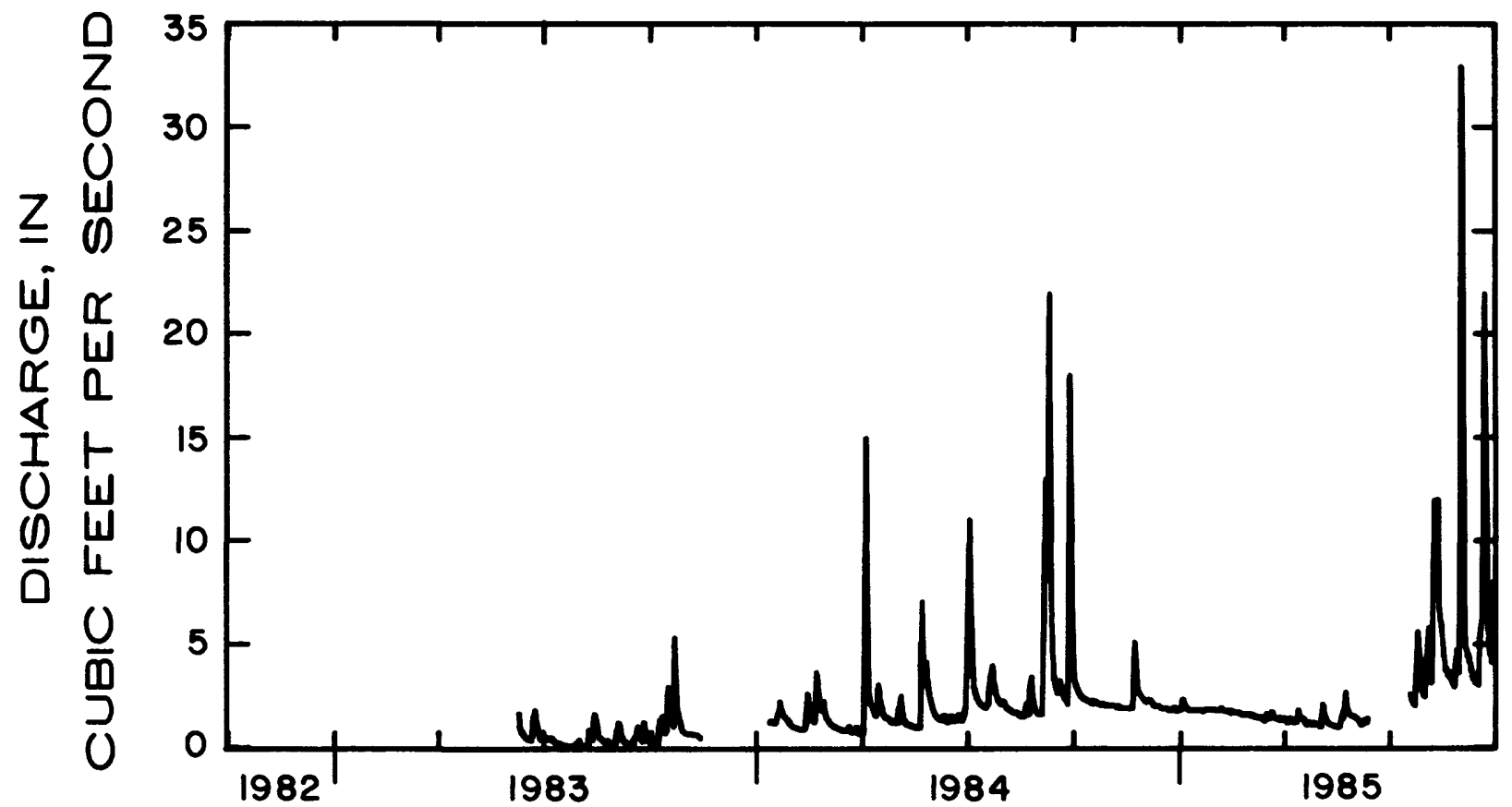

Figure 5. Daily discharges from October 1982 through September 1985 for site 3, Williamson Boulevard Ditch at Daytona Beach.

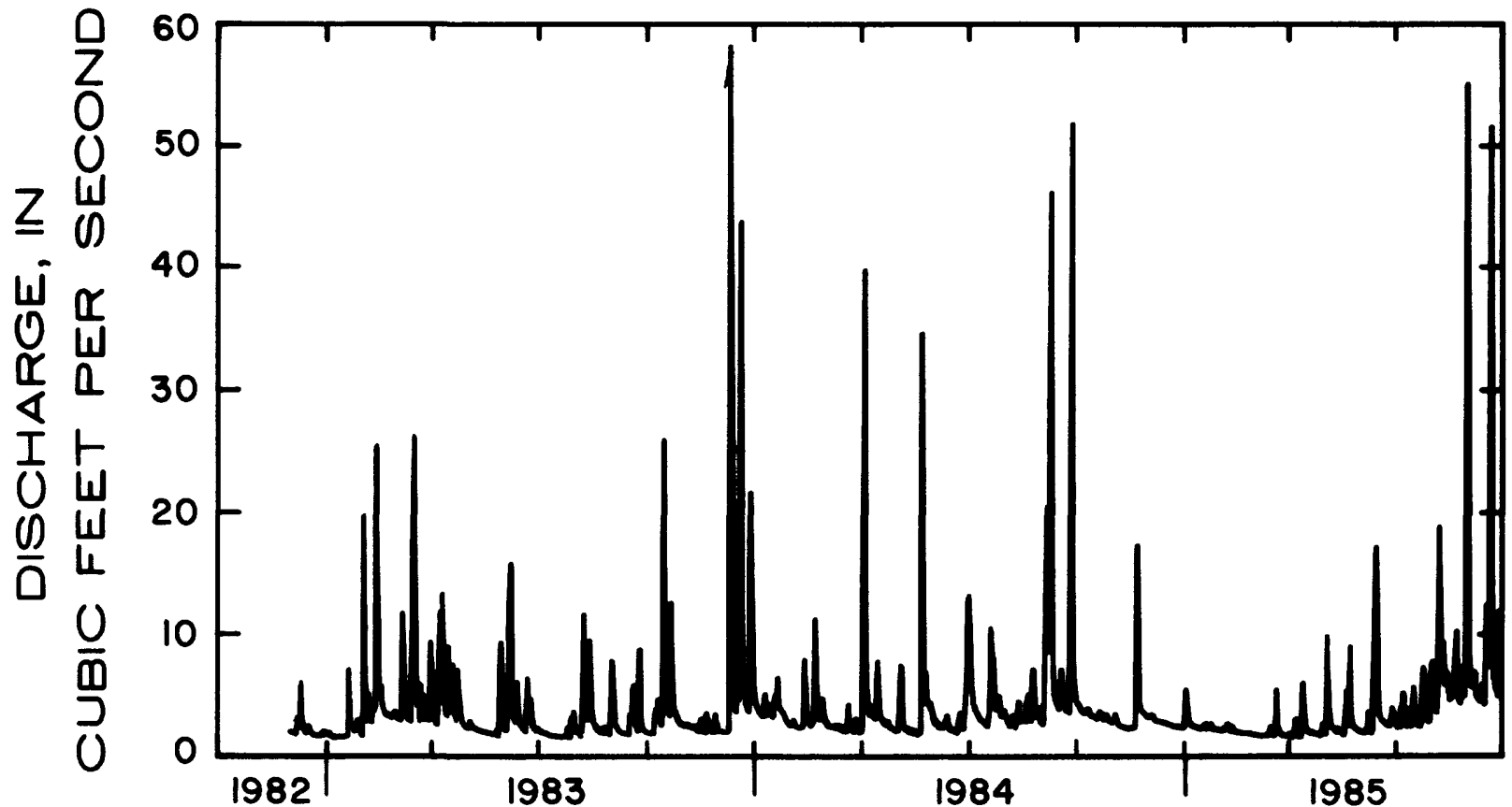

Figure 6. Daily discharges from October 1982 through September 1985 for site 4, Wally Hoffmeyer Canal at Daytona Beach. 


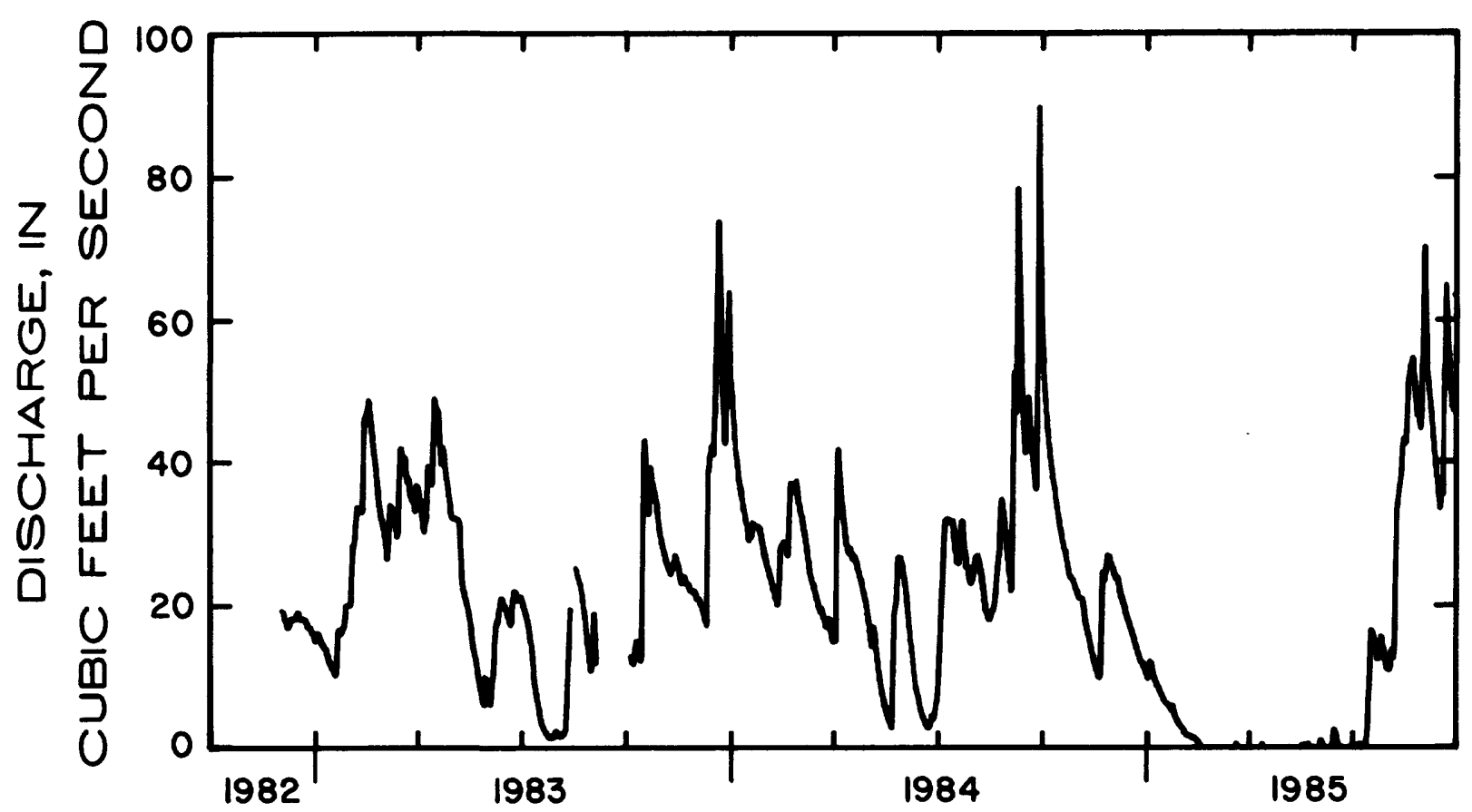

Figure 7. Daily discharges from October 1982 through September 1985 for site 5, Thayer Canal near Daytona Beach.

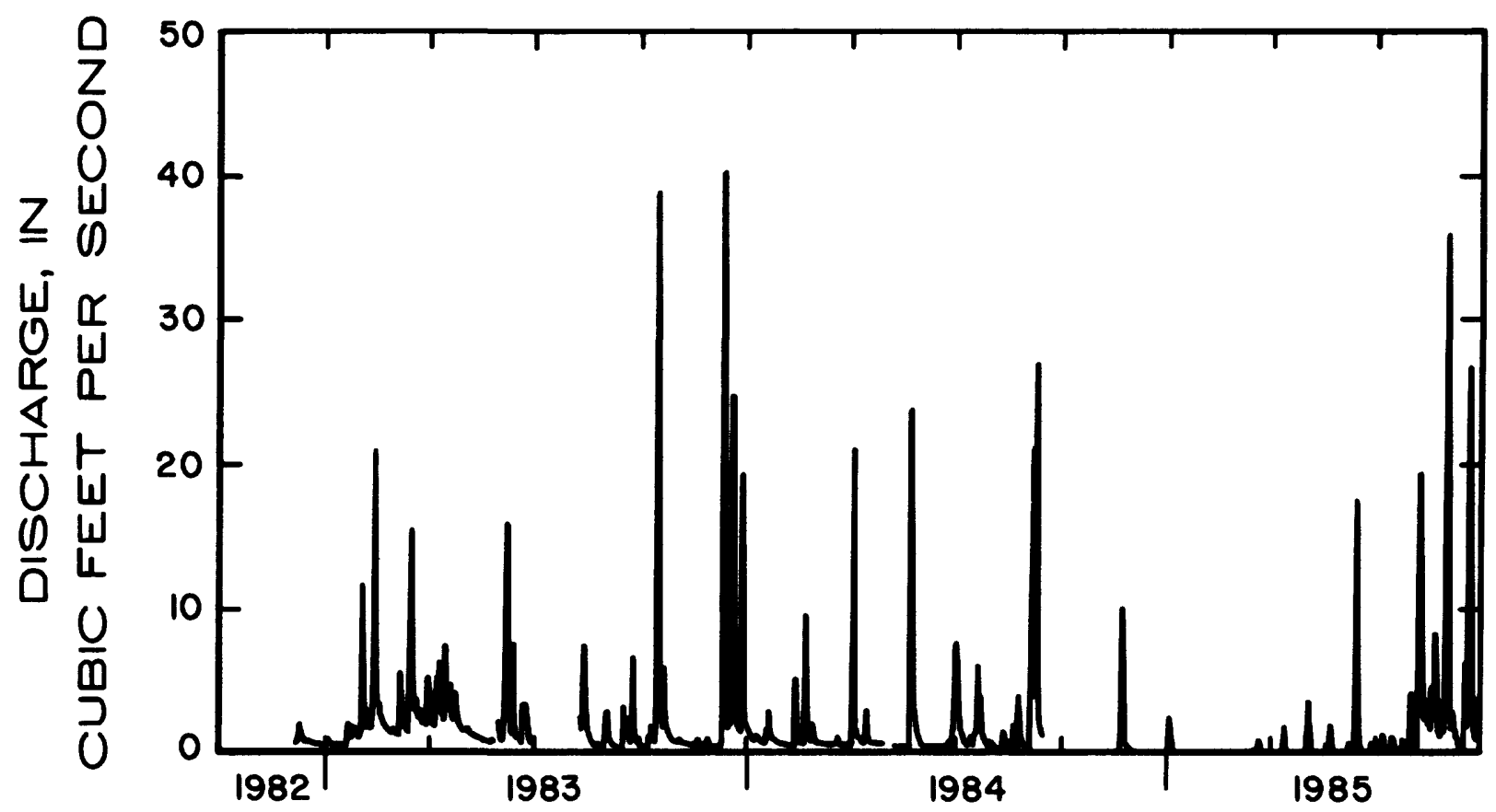

Figure 8. Daily discharges from October 1982 through September 1985 for site 6, Bayless Boulevard Canal at Daytona Beach. 


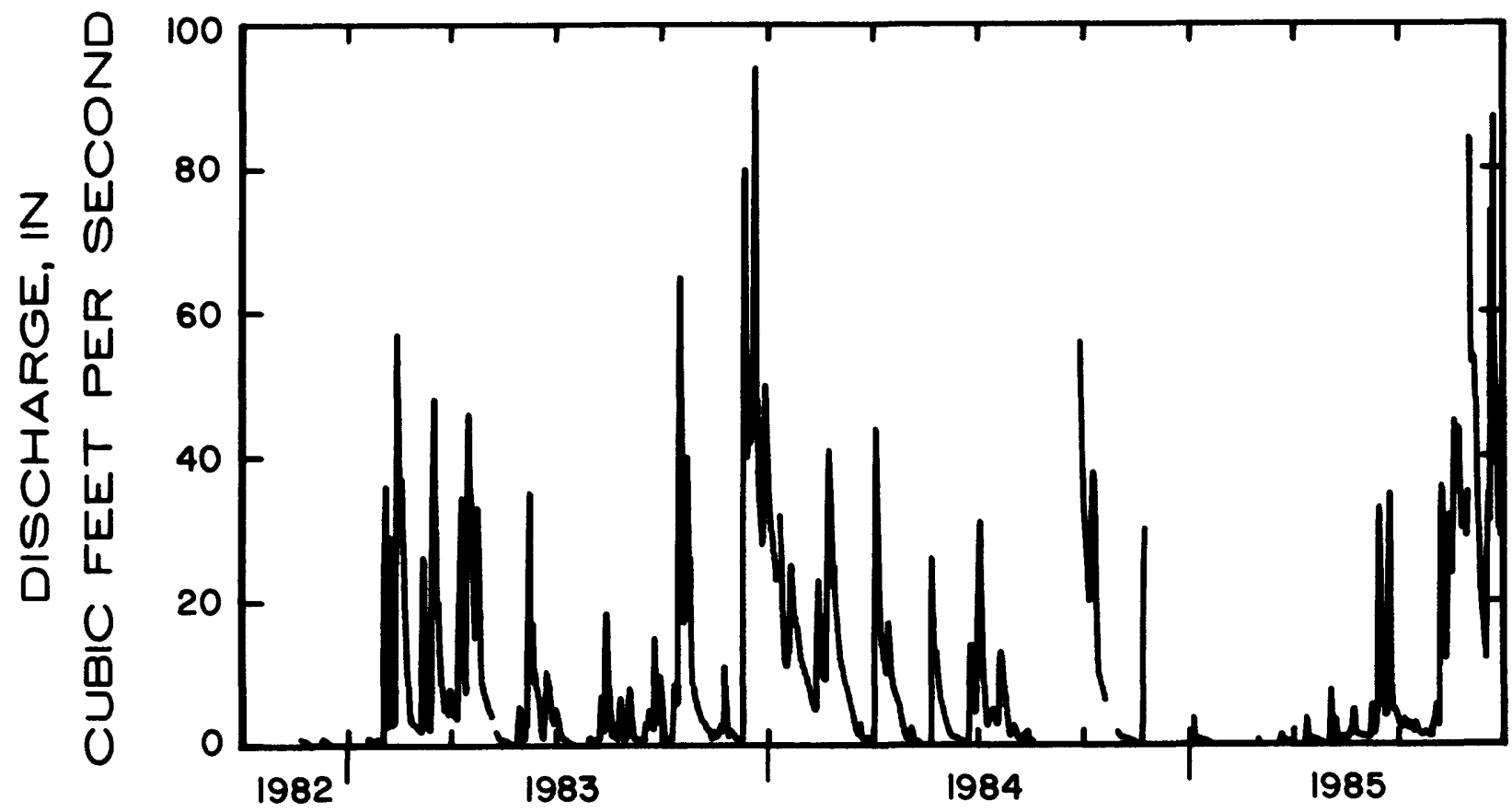

Figure 9. Daily discharges from October 1982 through September 1985 for site 7, Bellevue Canal at Daytona Beach.

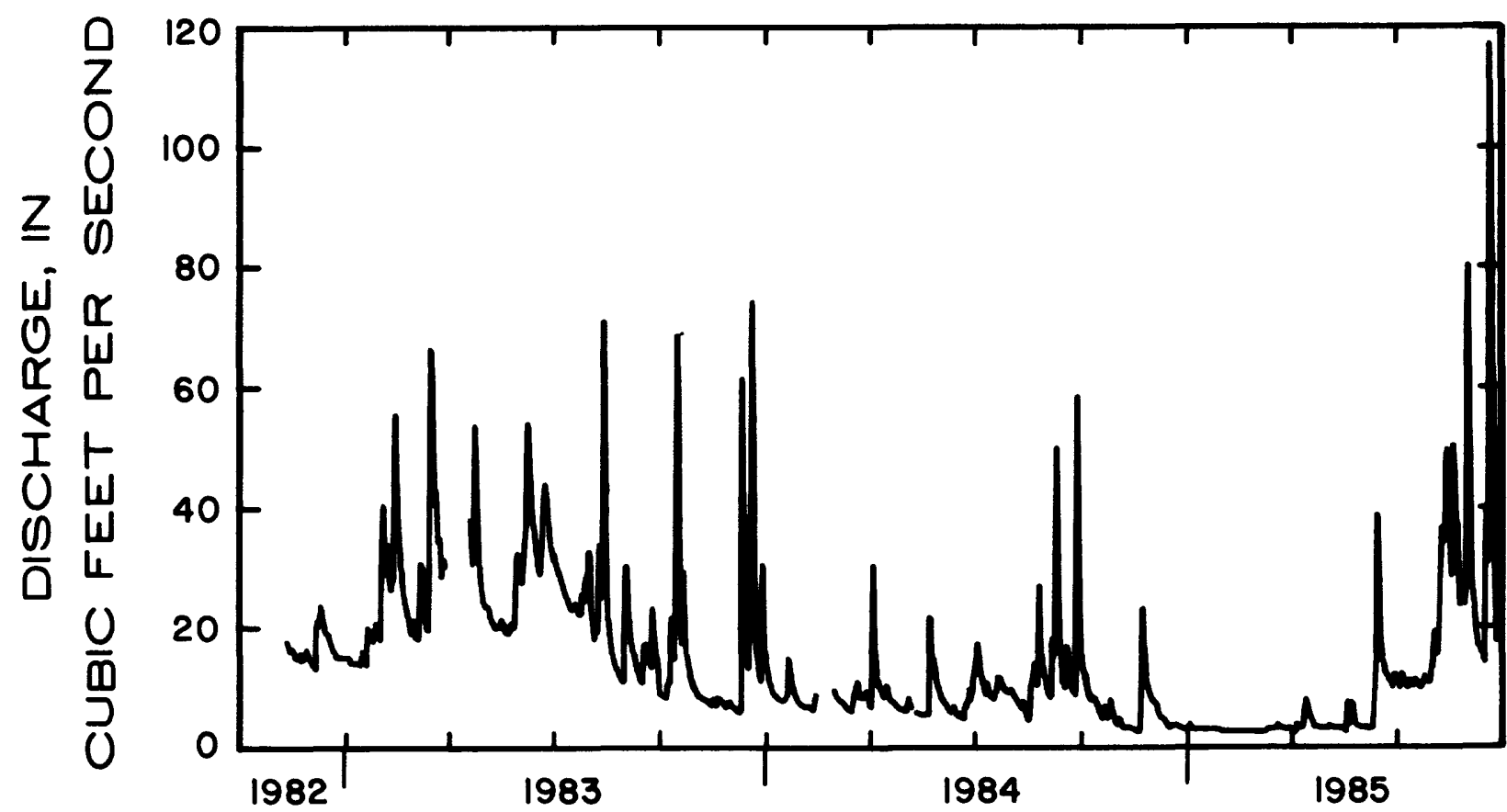

Figure 10. Daily discharges from October 1982 through September 1985 for site 11, B-19 Canal at Willow Run Boulevard at Port Orange. 


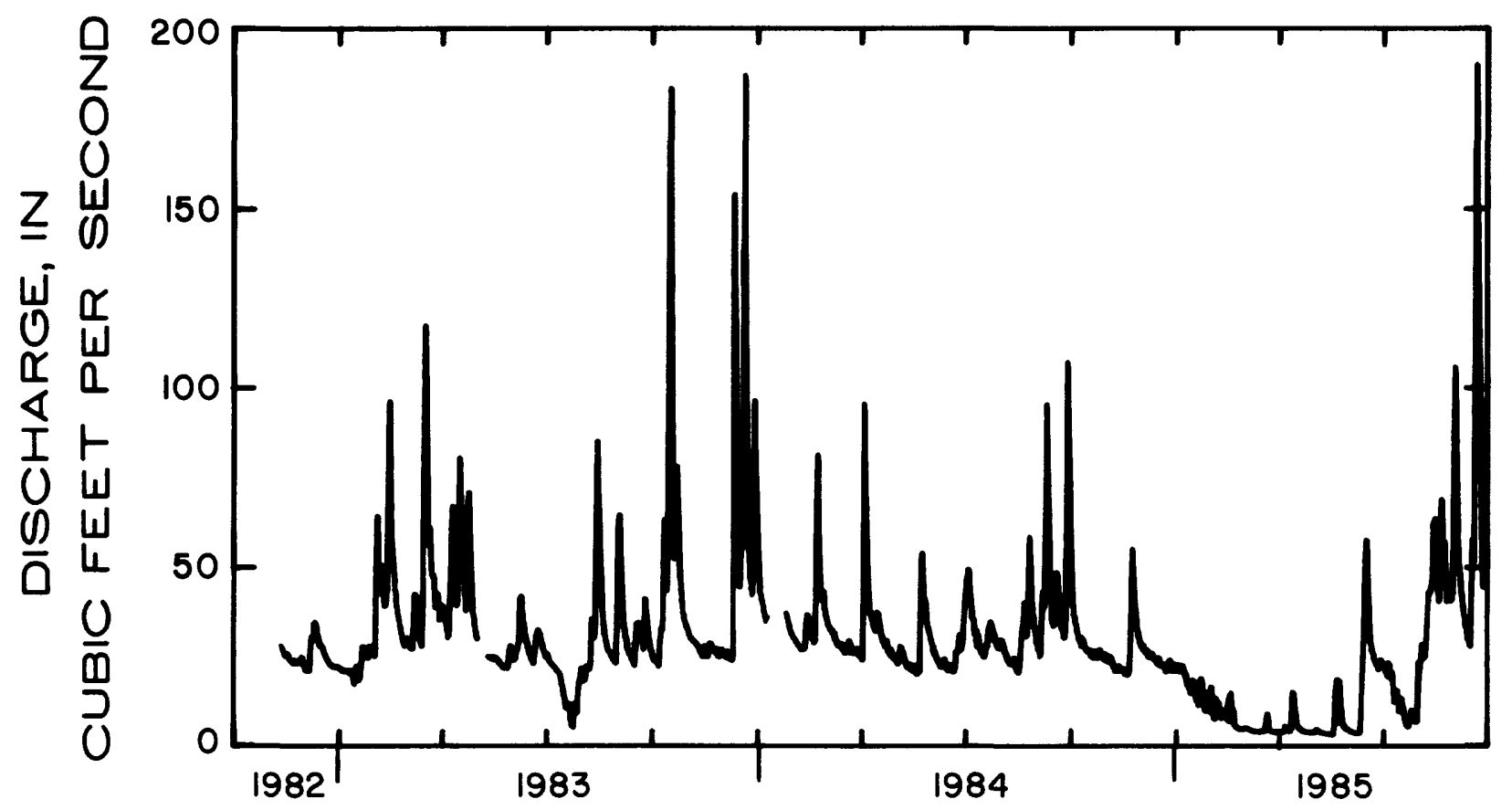

Figure 11. Daily discharges from October 1982 through September 1985 for site 12, B-19 Canal at Dunlawton Avenue at Port Orange.

At site 2 (Eleventh Street Canal near Holly Hill, drainage area $=3.5 \mathrm{mi}^{2}$ ), the same storm produced a peak in 9 hours (fig. 13), and it took about 68 hours for the canal to return to base-flow condition.

Comparing two different storms of about the same size for the same site shows the effect of antecedent basin conditions on the discharge hydrograph. For example, the storms of September 27, 1984, and September 1, 1985, (fig. 14) at site 3 (Williamson Boulevard Ditch at Daytona Beach), both had between 4 and 5 inches of rainfall. When the basin soils are dry at the start of a storm, as on September 27, 1984, the hydrographic response is slower and the peak discharge is lower. When the basin soils are wet at the start of a storm, as on September 1,1985 , the response of the hydrograph is much faster and the peak discharge is higher.

Rainfall and runoff data for selected storms with a magnitude of 2 inches or greater, including those storm data shown in figures $12-20$, are summarized in table 2 . This table includes data for rainfall as depth in inches, storm runoff as volume in cubic feet and depth in inches uniformly distributed over the basin, and runoff as a percentage of rainfall. Total equivalent rainfall was determined from nearby rainfall sites using the Thiessen polygon method and is expressed as equivalent depth in inches over the entire basin (Gilman, 1964).
Storm runoff was computed from storm hydrographs by subtracting base flow from total discharge. Base flow was determined graphically by extending a line from prestorm base flow to an inflection point of the recession curve (see fig. 12). Plots of the rainfall-runoff data appear in figures 21-29.

In the Tomoka River basin, runoff generally was less than 2 inches and the ratio of runoff to rainfall varied widely, ranging from 1 to 77 percent for the storms documented in table 2. Site 2 (Eleventh Street Canal near Holly Hill) seems to have the most consistent ratios. In the B-19 Canal basin, runoff generally was less than 1 inch and varied between 6 and 17 percent of the total rainfall and showed little consistency. In general, ratios were widely varied and the rainfall-runoff plots appear to have few discernible patterns.

Variations in runoff values for the B-19 Canal are believed to be due, in part, to reversed flow and storage from backwater conditions caused by changes in channel geometry, such as culvert constrictions. Reverse flow was observed a number of times at site 10 (B-19 Canal near Daytona Beach). Variations in runoff values for the Tomoka River basin are believed partly due to interconnected channels that allow flow in either direction depending on the hydraulic gradient. Large marshy areas in this basin tend to allow storage and evaporation, thereby retarding or reducing runoff. Some runoff leaves the basin as an increase in base flow due to this retarding effect. 

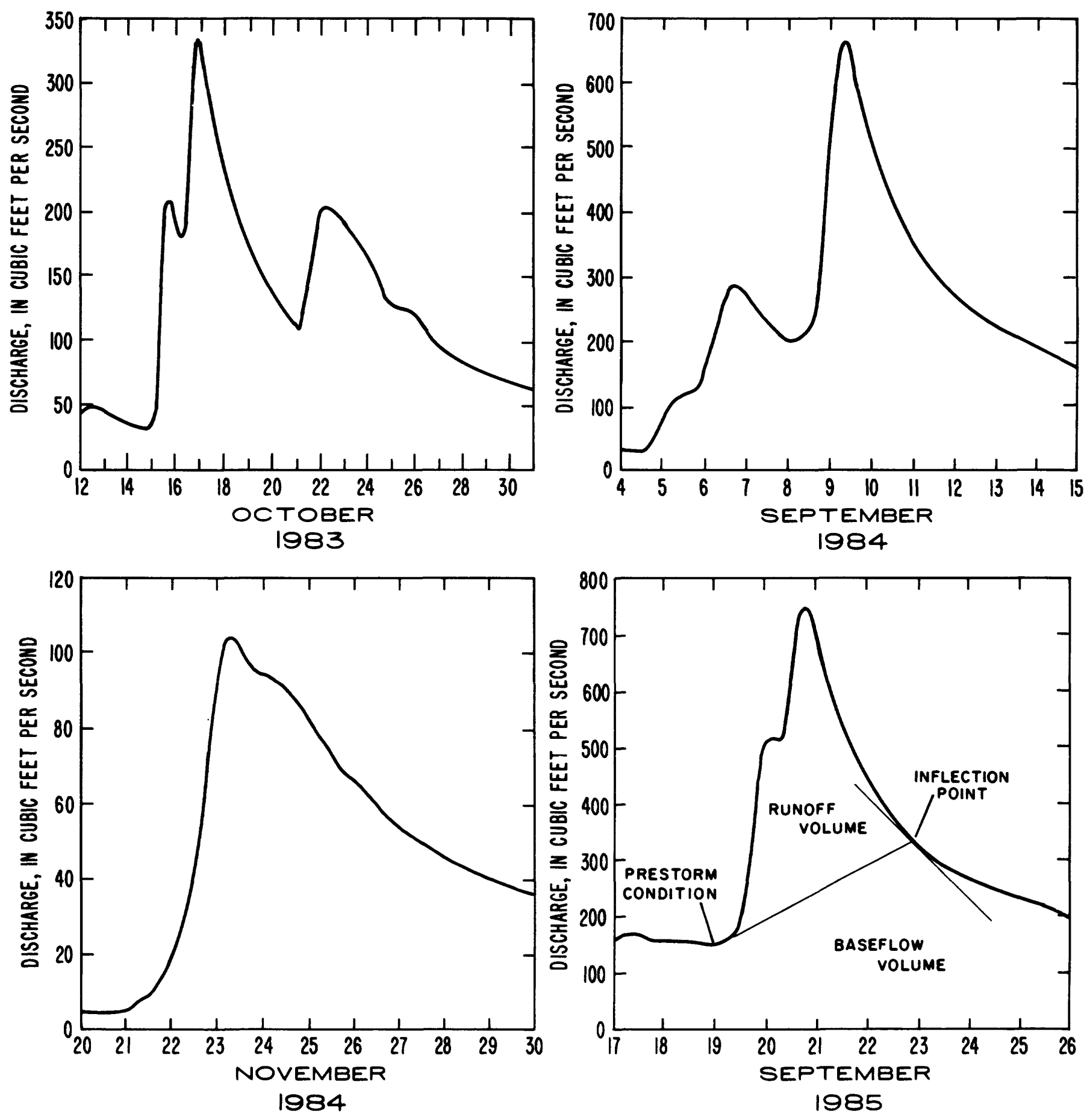

Figure 12. Selected storms at site 1, Tomoka River near Holly Hill. 

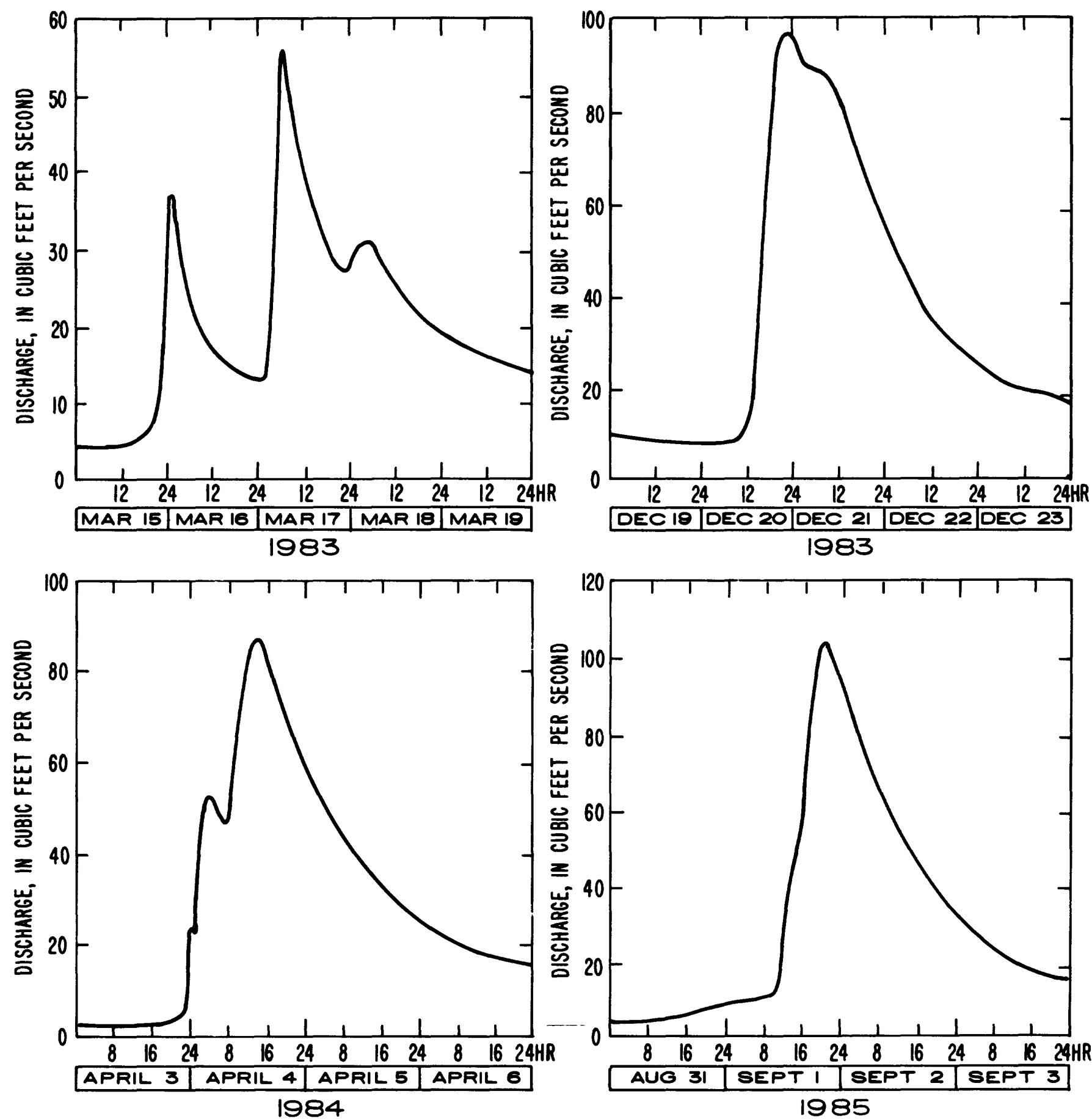

Figure 13. Selected storms at site 2, Eleventh Street Canal near Holly Hill. 

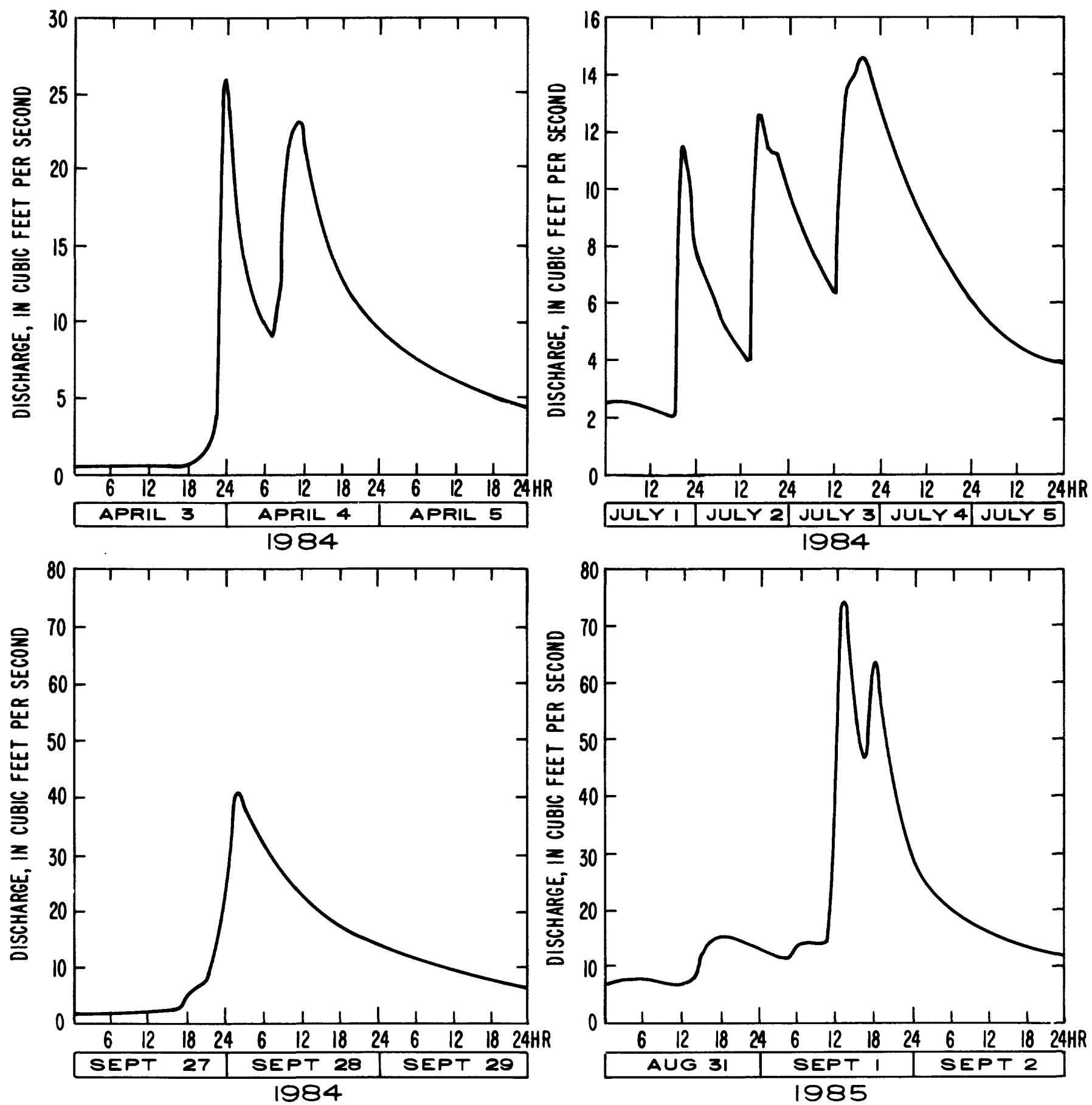

Figure 14. Selected storms at site 3, Williamson Boulevard Ditch at Daytona Beach. 

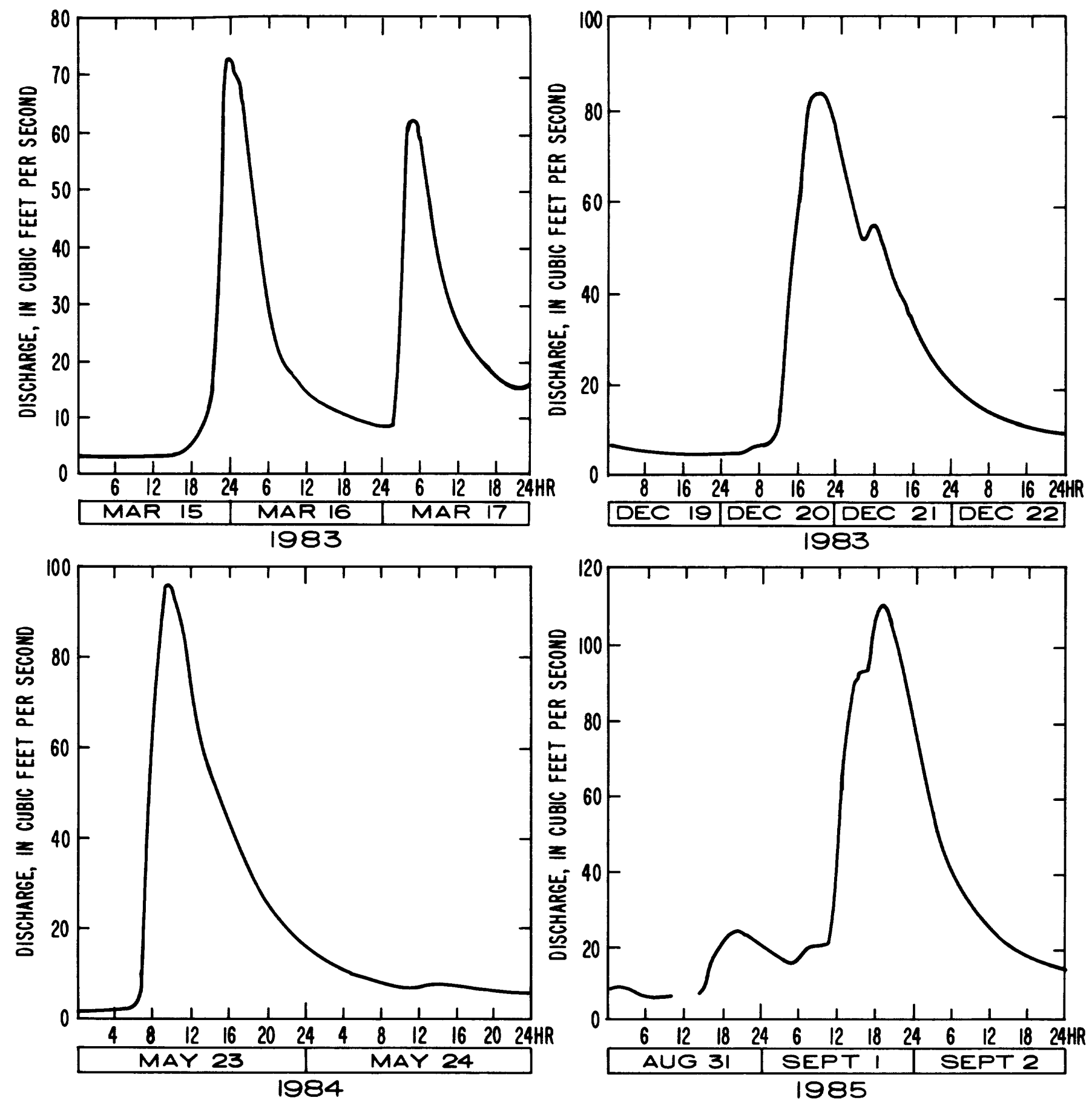

Figure 15. Selected storms at site 4, Wally Hoffmeyer Canal at Daytona Beach. 

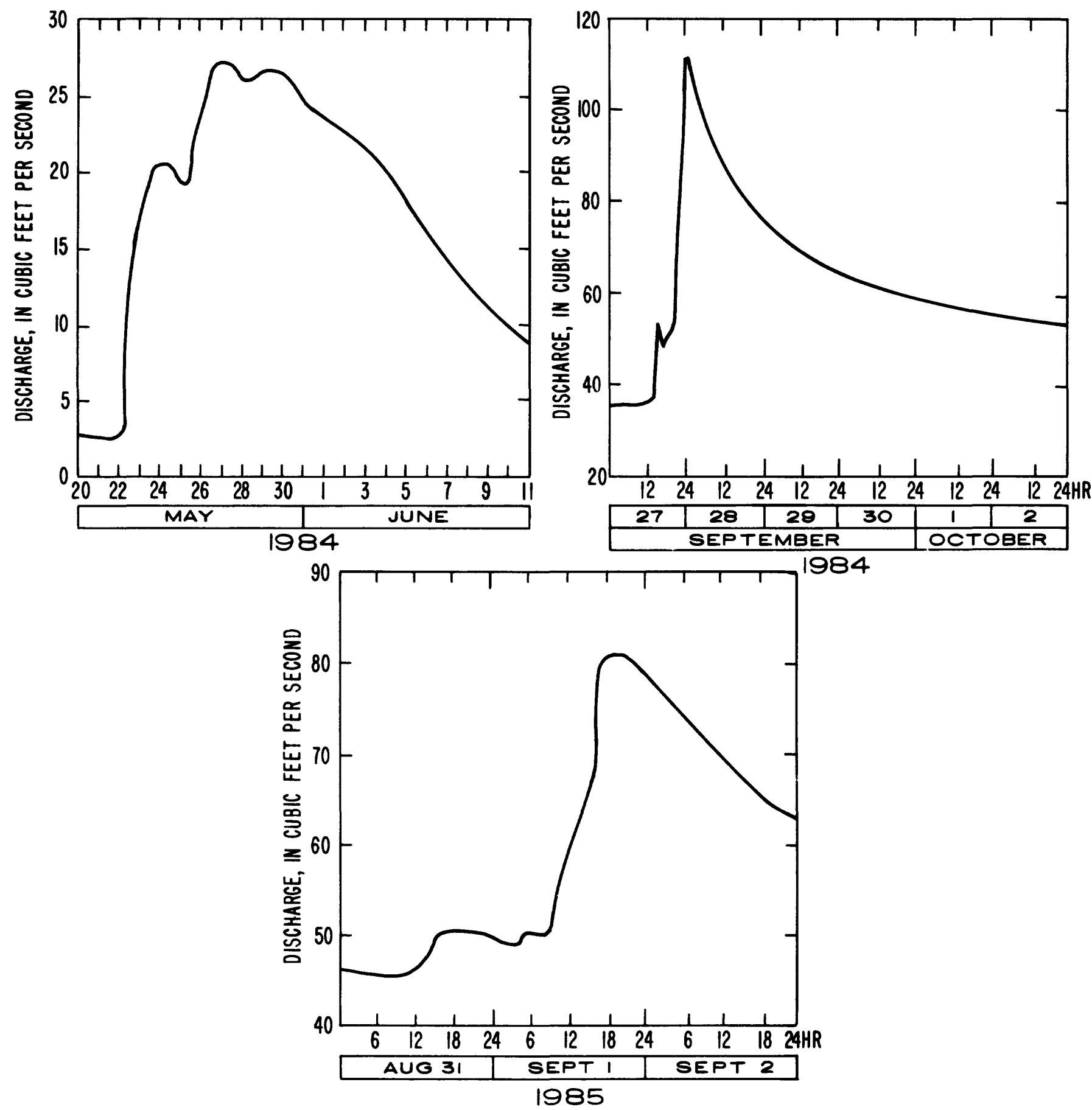

Figure 16. Selected storms at site 5, Thayer Canal near Daytona Beach. 

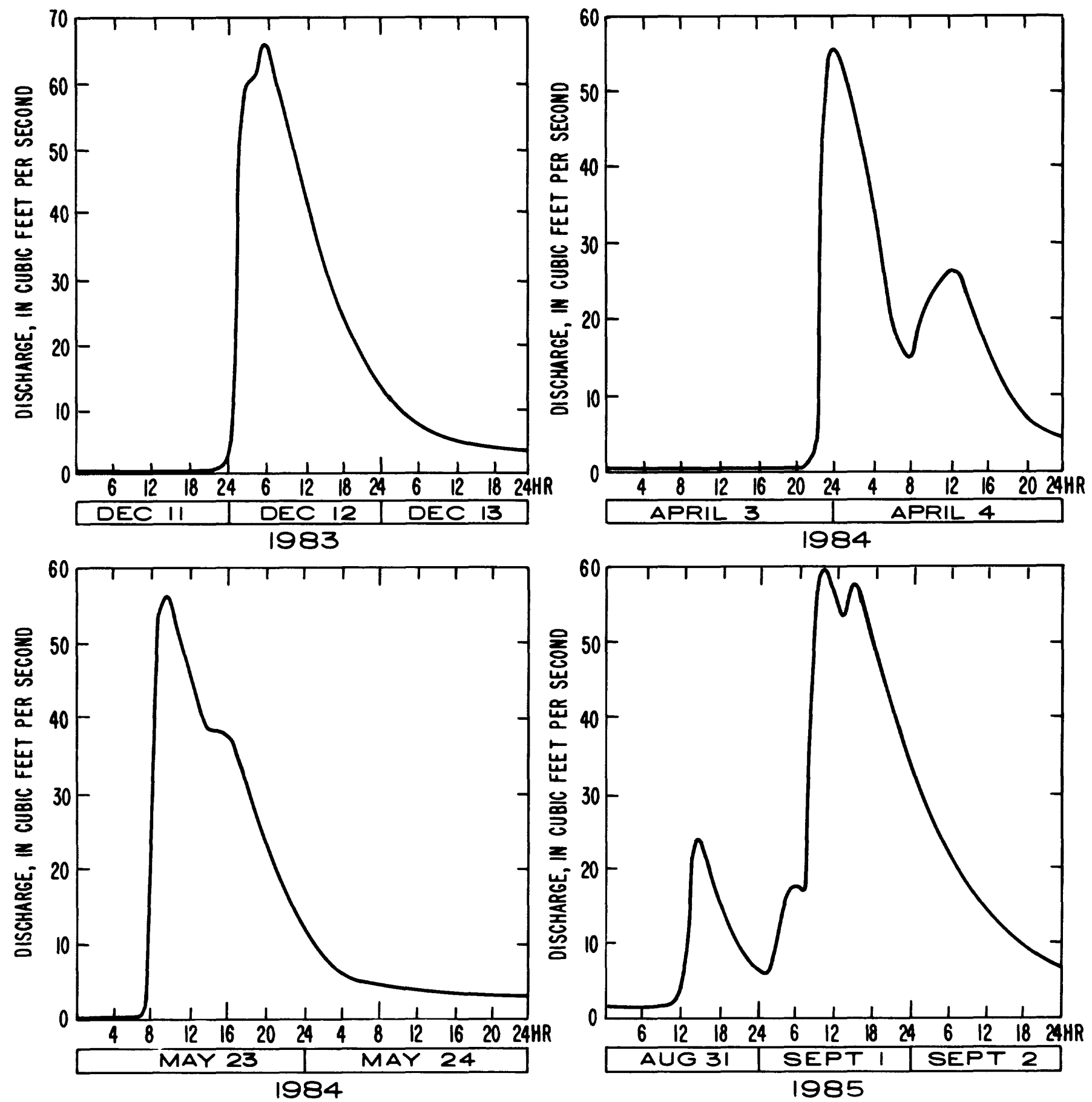

Figure 17. Selected storms at site 6, Bayless Boulevard Canal at Daytona Beach. 

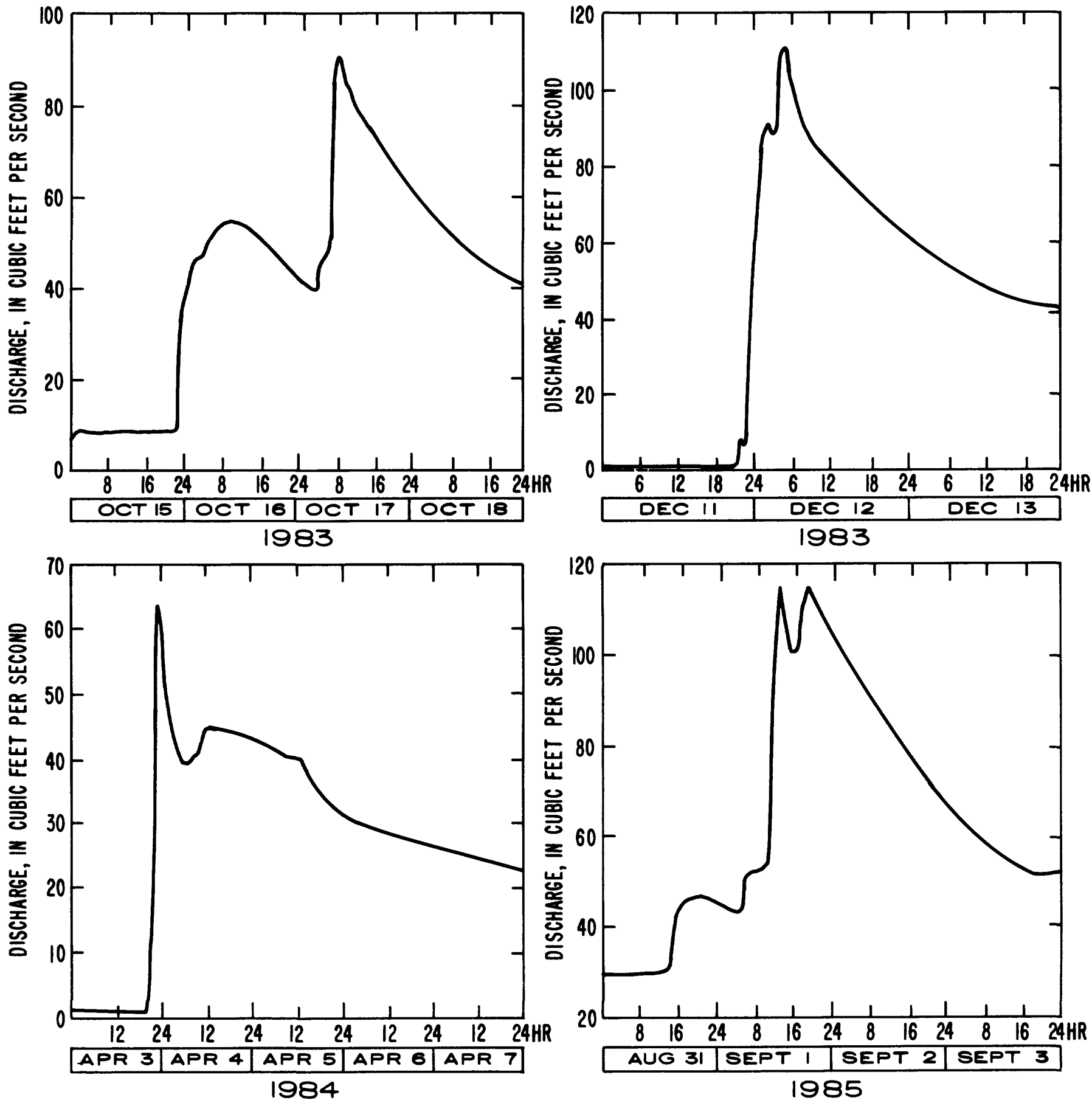

Figure 18. Selected storms at site 7 , Bellevue Canal at Daytona Beach. 

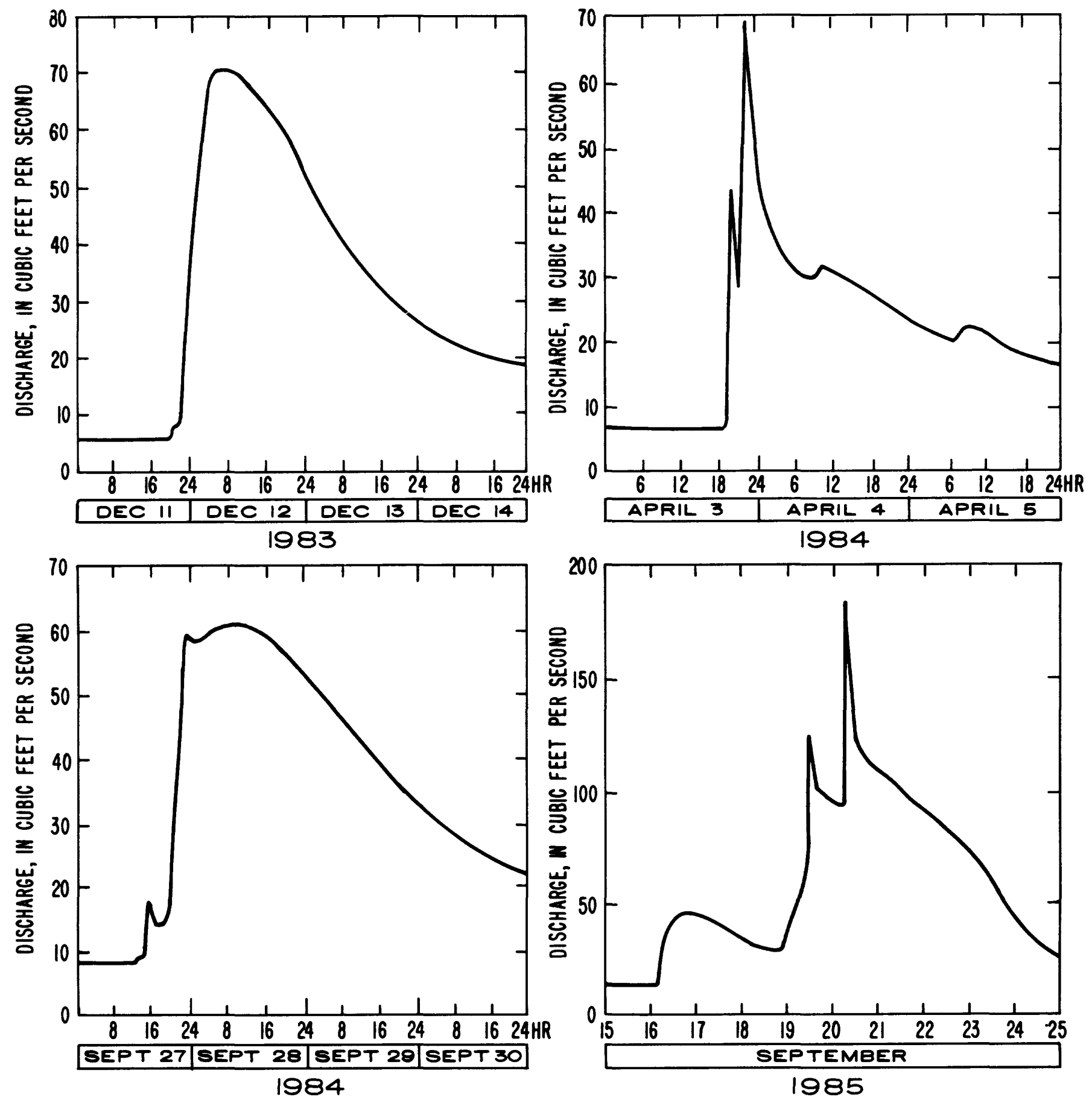

Figure 19. Selected storms at site 11, B-19 Canal at Willow Run Boulevard at Port Orange. 

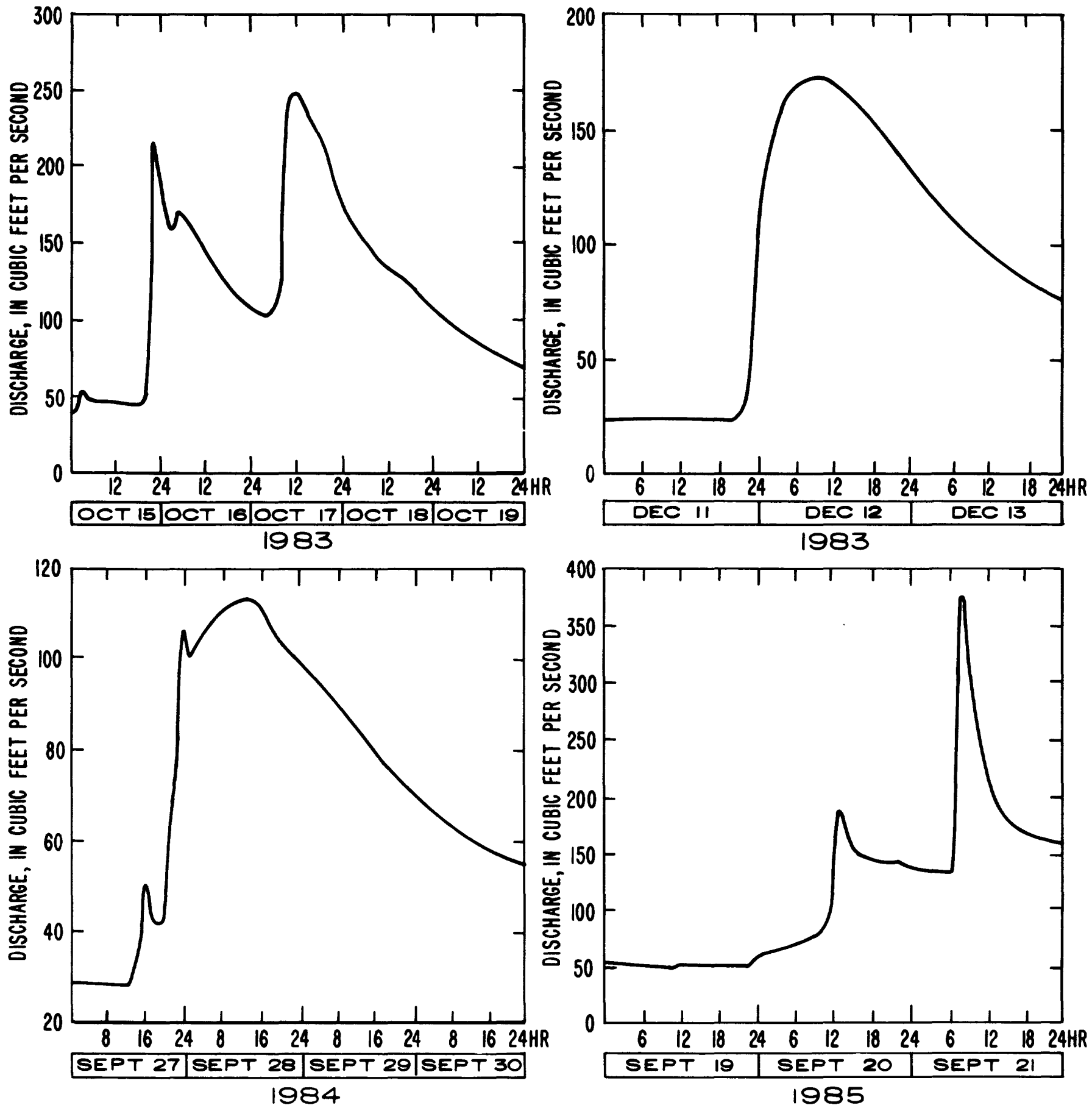

Figure 20. Selected storms at site 12, B-19 Canal at Dunlawton Avenue at Port Orange. 
Table 2. Summary of Thiessen-method rainfall and resulting runoff for selected storms [ $\mathrm{it}^{3}$, cubic feet]

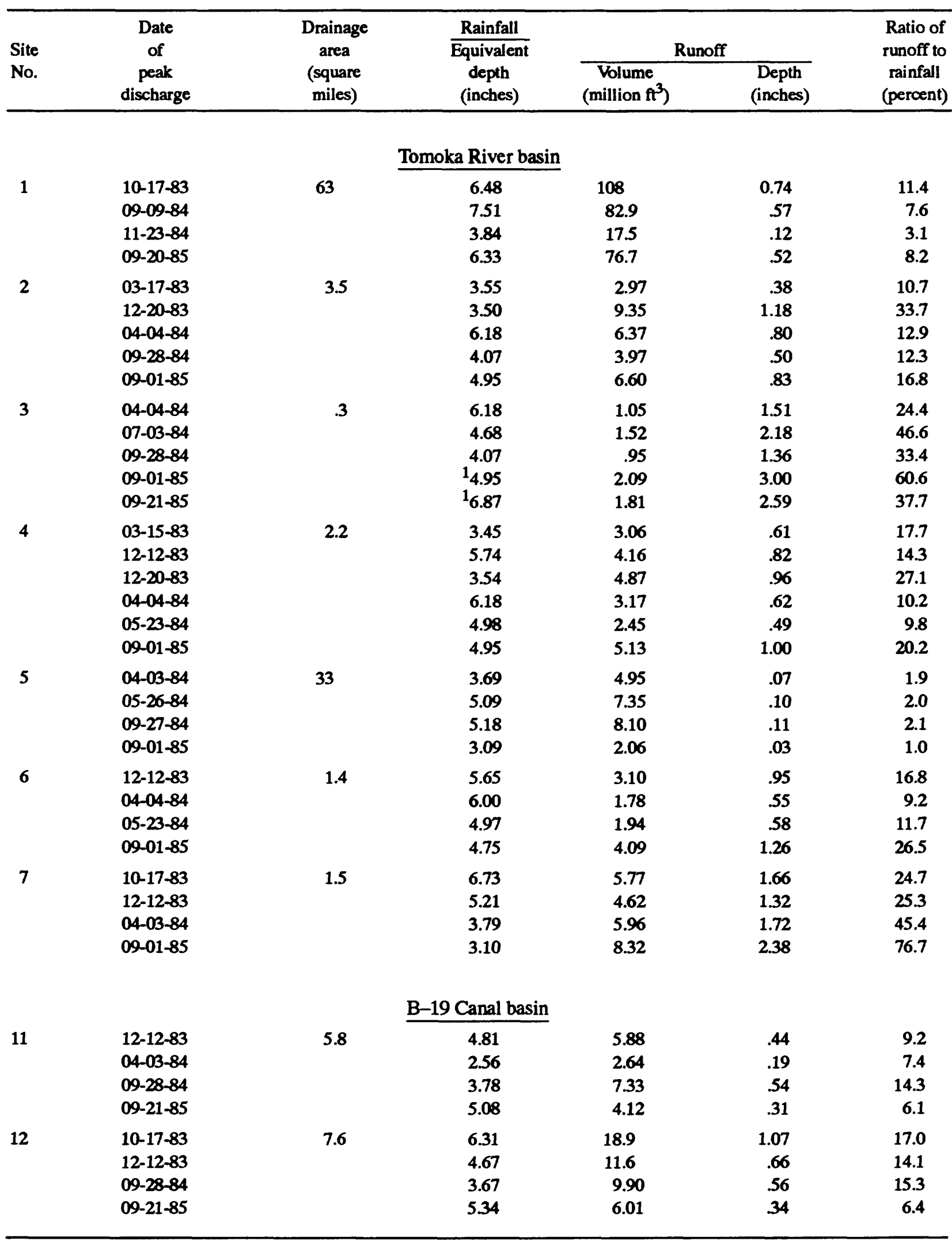

${ }^{1}$ Estimated. 


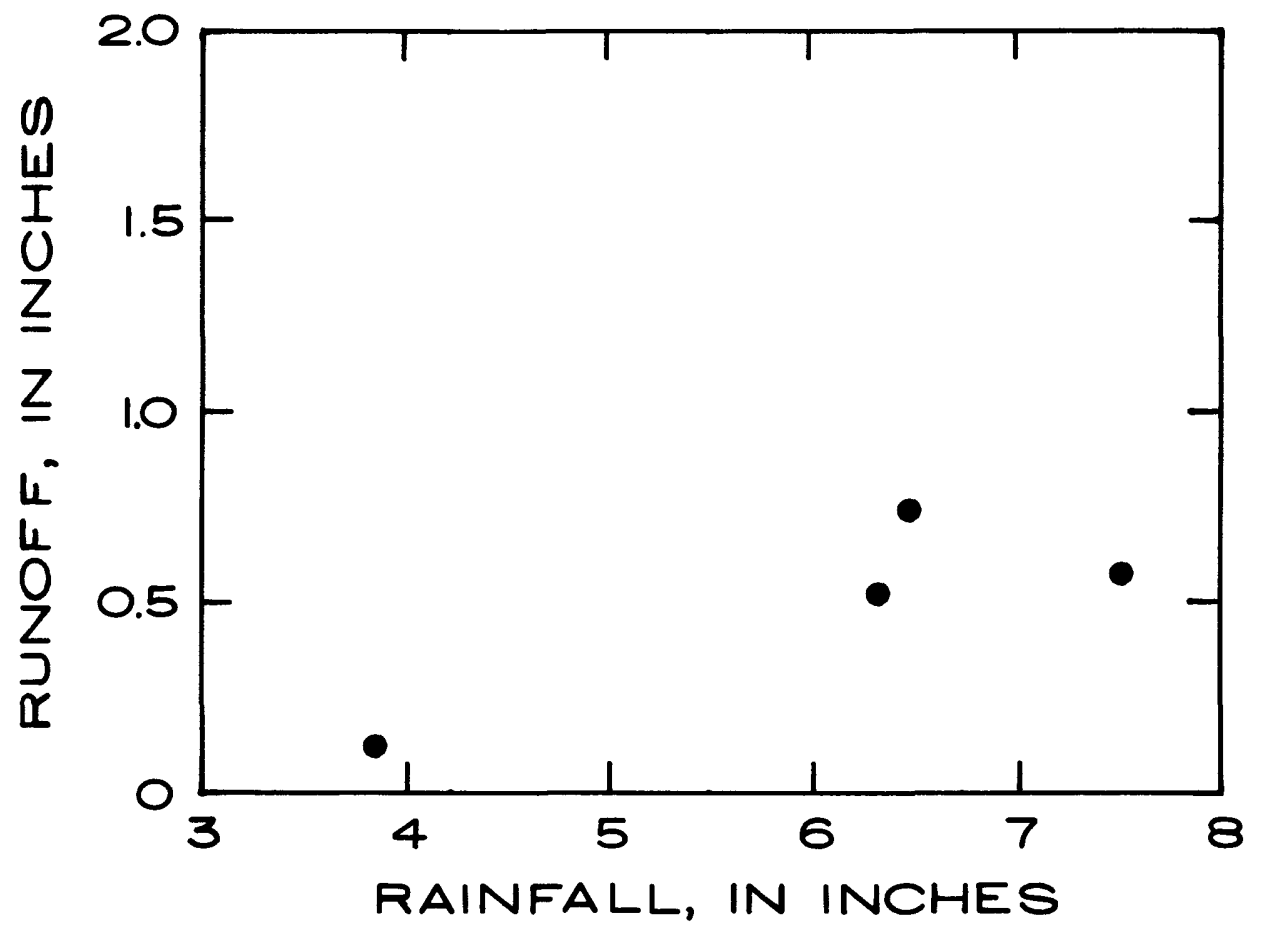

Figure 21. Rainfall-runoff plots for site 1, Tomoka River near Holly Hill.

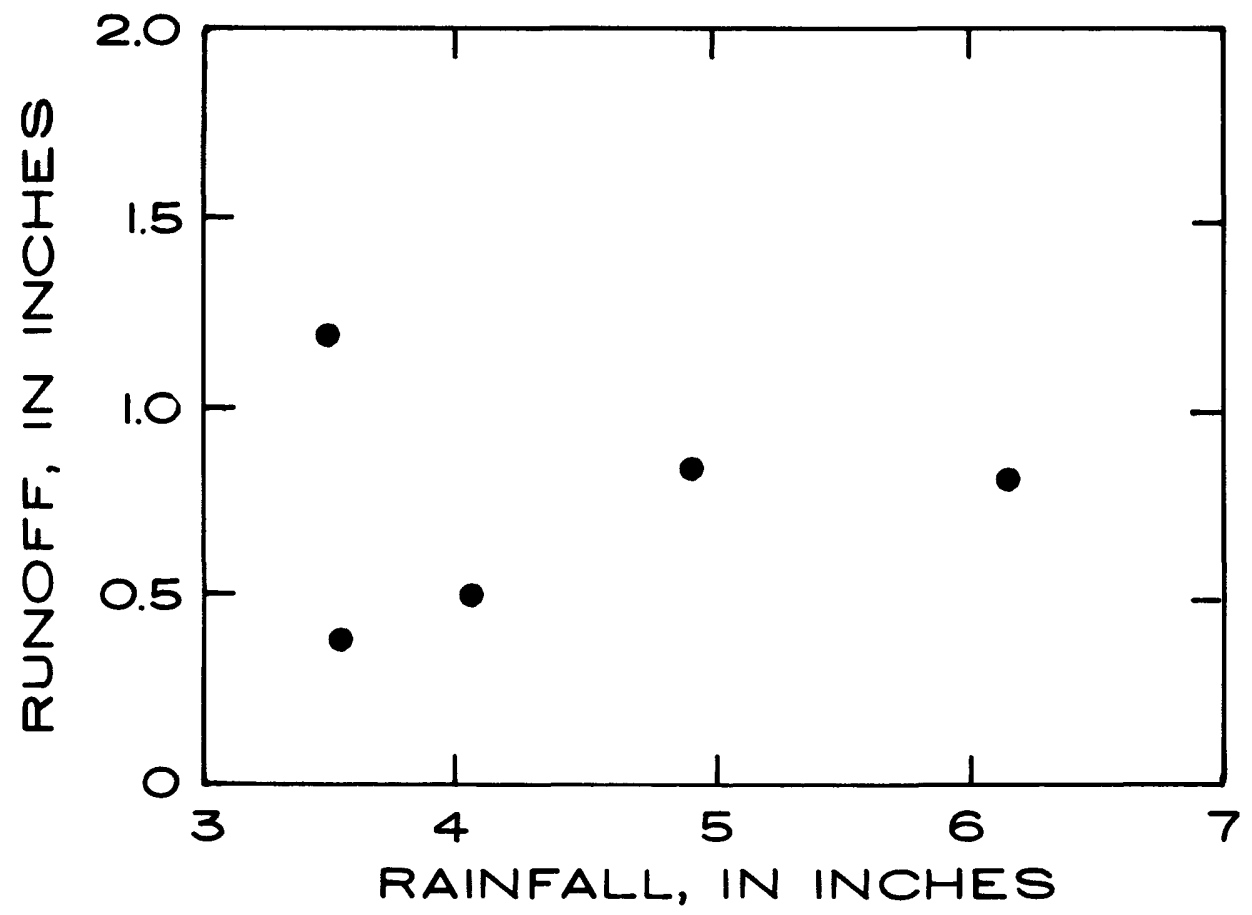

Figure 22. Rainfall-runoff plots for site 2, Eleventh Street Canal near Holly Hill. 


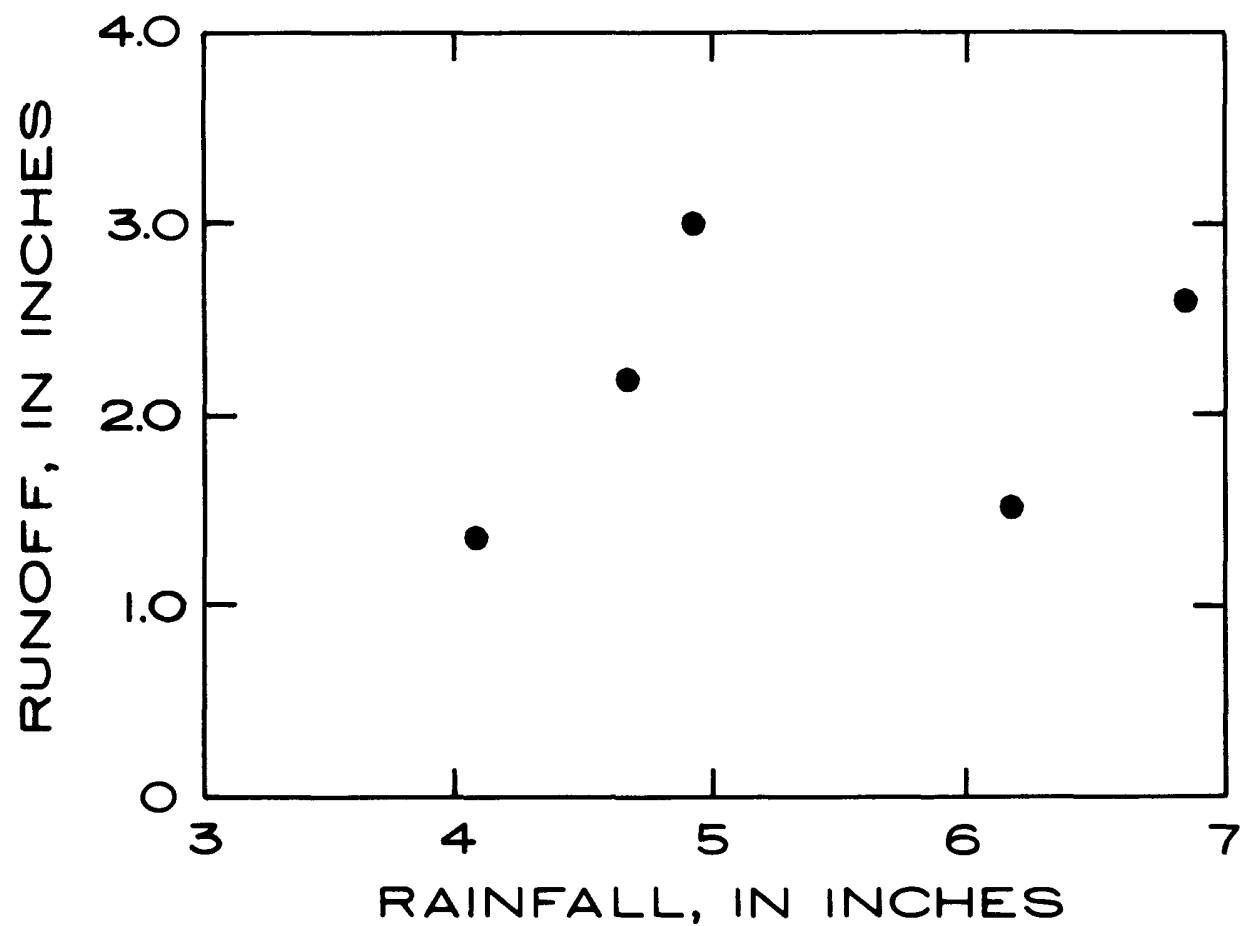

Figure 23. Rainfall-runoff plots for site 3, Williamson Boulevard Ditch at Daytona Beach.

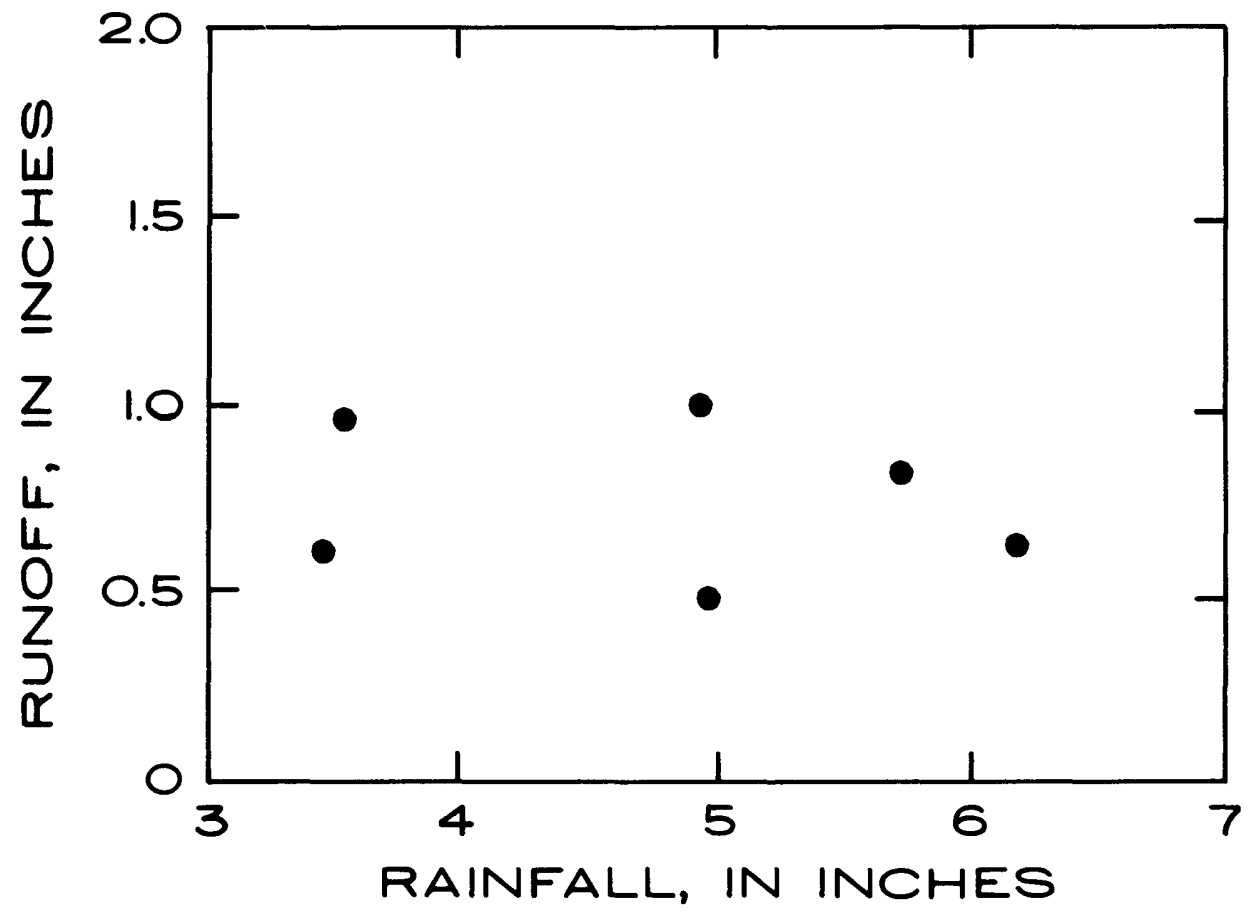

Figure 24. Rainfall-runoff plots for site 4, Wally Hoffmeyer Canal at Daytona Beach. 


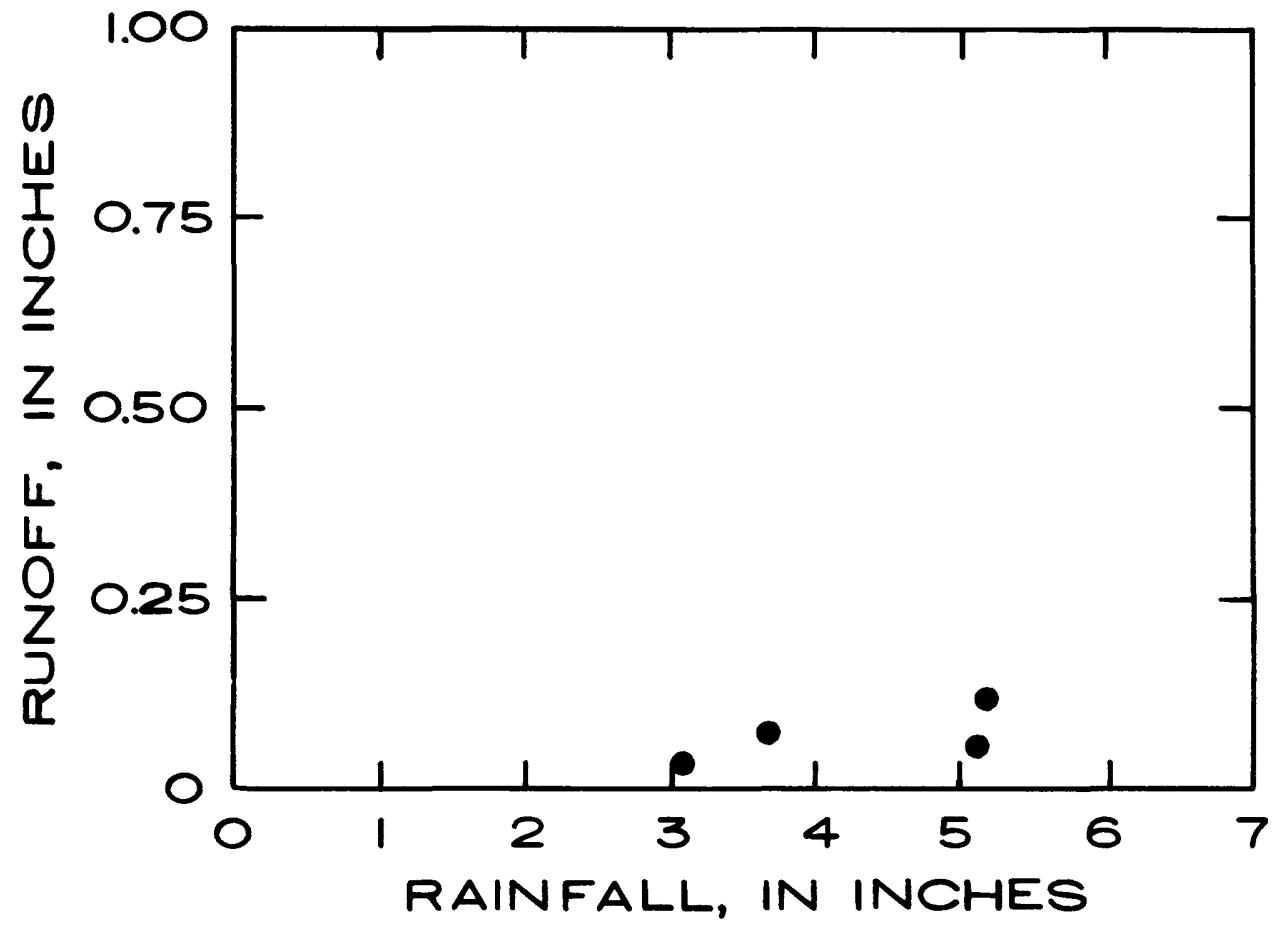

Figure 25. Rainfall-runoff plots for site 5, Thayer Canal near Daytona Beach.

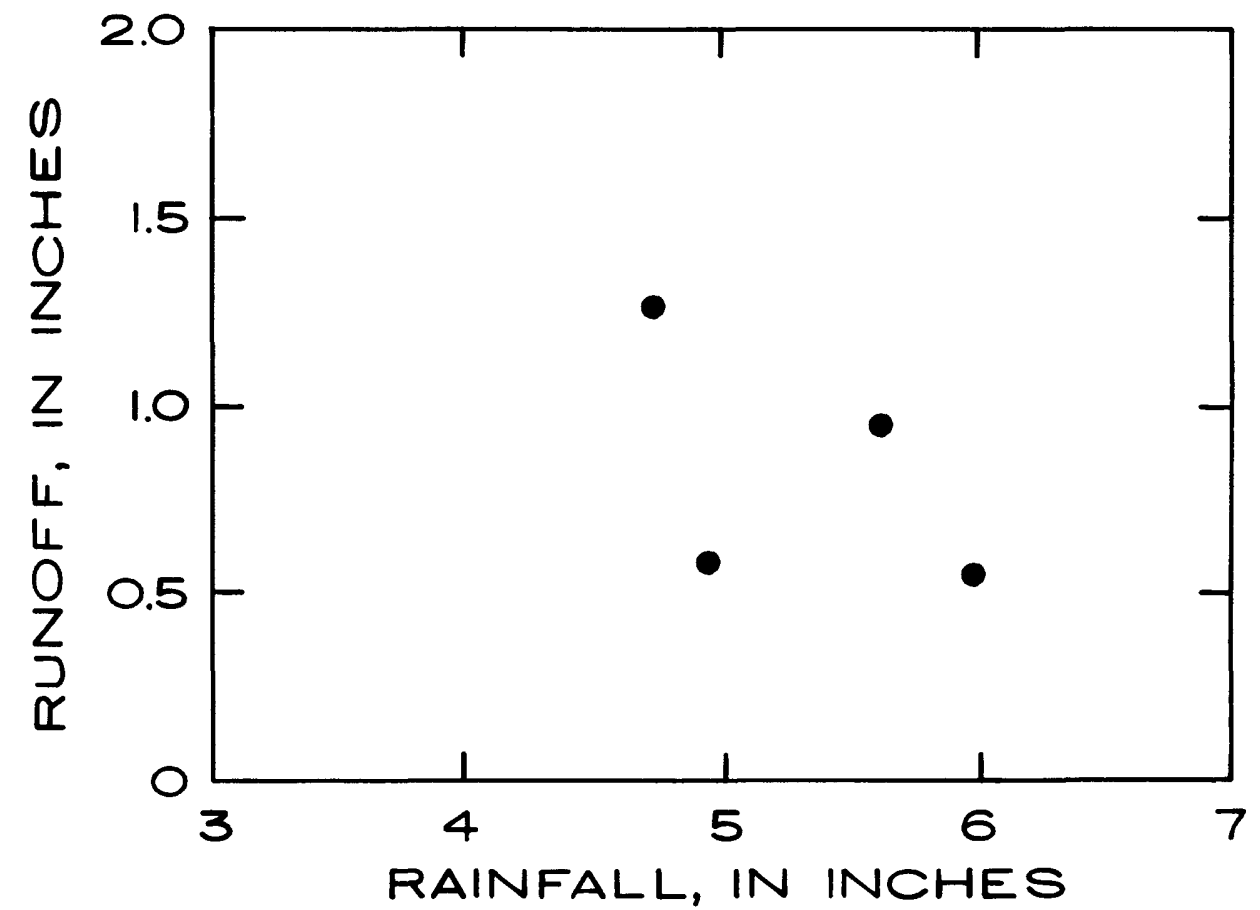

Figure 26. Rainfall-runoff plots for site 6, Bayless Boulevard Canal at Daytona Beach. 


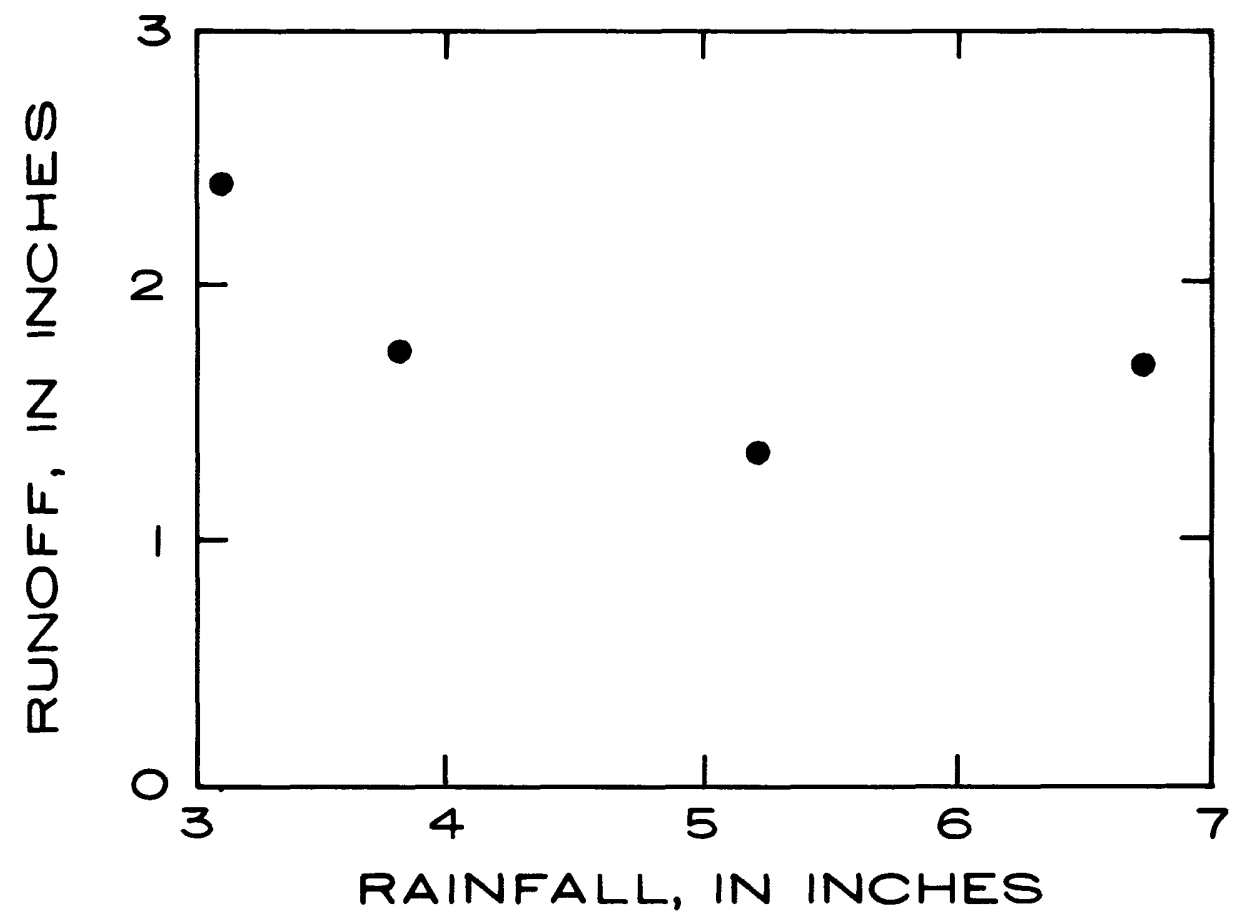

Figure 27. Rainfall-runoff plots for site 7, Bellevue Canal at Daytona Beach.

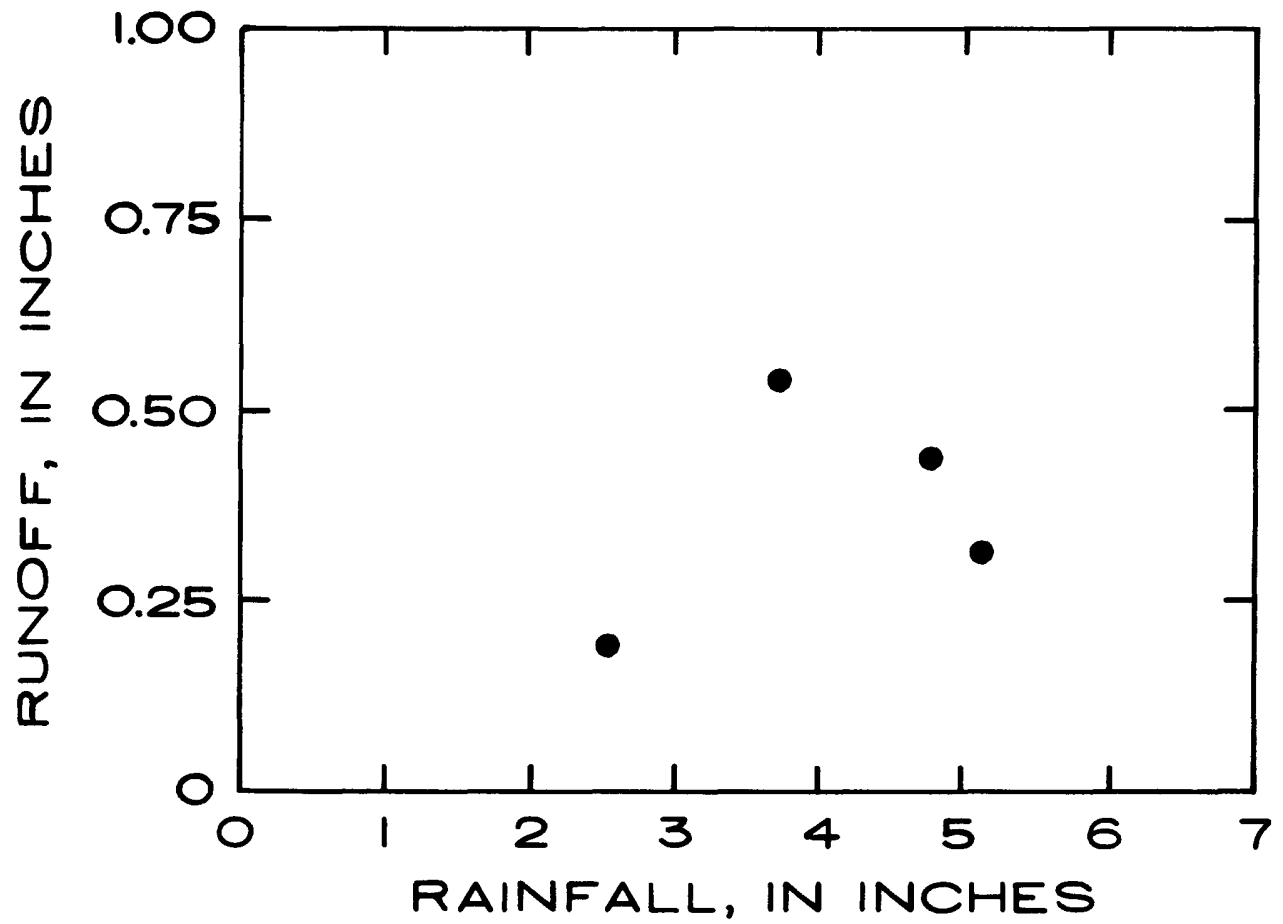

Figure 28. Rainfall-runoff plots for site 11, B-19 Canal at Willow Run Boulevard at Port Orange. 


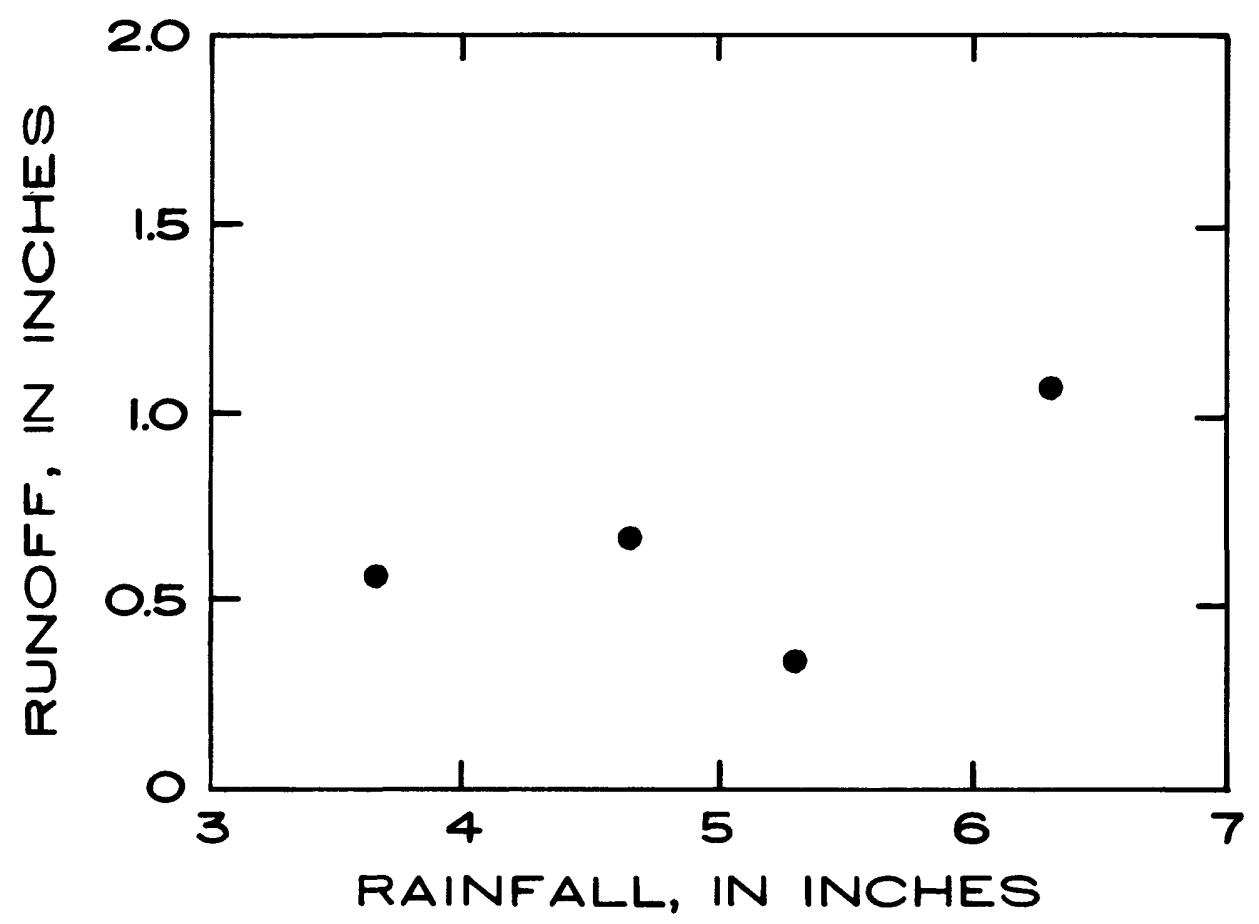

Figure 29. Rainfall-runoff plots for site 12, B-19 Canal at Dunlawton Avenue at Port Orange.

\section{QUALITY OF STORMWATER RUNOFF}

The report "Tomoka River Watershed Water Quality Study" (Volusia County Planning Board, 1978) shows the following ranges of constituents concentrations:

[Units are in milligrams per liter, except where noted;

$<$, less than; col $/ 100 \mathrm{~mL}$, colonies per 100 milliliters]

\begin{tabular}{|c|c|}
\hline Constituent or property & Range \\
\hline pH (units) & $6.4 \overline{5-7.4}$ \\
\hline Total solids & $06-303$ \\
\hline Suspended solids & $<1-21$ \\
\hline Total nitrogen & $.62-1.29$ \\
\hline Total phosphorus & $.03-0.29$ \\
\hline Orthophosphate (as P) & $.01-0.2$ \\
\hline Dissolved oxygen & $3.2-8.6$ \\
\hline Coliform bacteria & $7,100-26,0$ \\
\hline
\end{tabular}

Ranges in concentrations of selected constituents for previously published studies and for samples collected during this study are given in table 3 . Also listed are limits for these constituents recommended by the USEPA and the FDER, or suggested by McKee and Wolf (1963) and the Federal Water Pollution Control Administration (1968). Limits for constituents or characteristics discussed in the text but not listed in table 3 are based on FDER class III surface waters, except where specified differently. These limits were used because the Tomoka River is classified as a class III waters by FDER.
Analyses of samples collected during this study show some differences from the 1978 Tomoka River watershed report. Of 26 samples collected in the Tomoka River basin during this study, only 1 total phosphorus concentration exceeded the previous maximum value reported, whereas 7 concentrations were below the previous minimum. Thirtythree percent of the samples analyzed had total phosphorus concentrations greater than the $0.05 \mathrm{mg} / \mathrm{L}$ suggested limit to prevent enormous plant growth in surface waters (Federal Water Pollution Control Administration, 1968). The median concentration was $0.04 \mathrm{mg} / \mathrm{L}$ (milligrams per liter). Of 10 samples taken from the B-19 Canal basin, 90 percent had total phosphorus concentrations greater than the $0.05 \mathrm{mg} / \mathrm{L}$ recommended limit. Two of these samples had concentrations greater than the previous 1978 maximum reported for the nearby Tomoka River basin. The median concentration for the B-19 Canal analyses was $0.16 \mathrm{mg} / \mathrm{L}$.

Orthophosphate (as phosphorus) concentrations reported during the present study also showed some differences from previous results. Two samples in the Tomoka River basin exceeded the previous maximum concentration for the Tomoka River basin, whereas all samples from the B-19 Canal were below the previous minimum. The median concentrations during this study were $0.01 \mathrm{mg} / \mathrm{L}$ for the Tomoka River basin, and $0.07 \mathrm{mg} / \mathrm{L}$ for the B-19 Canal basin. 
During the present study, total nitrogen concentrations for samples collected ranged from 0.09 to $2.4 \mathrm{mg} / \mathrm{L}$. Eleven percent of the samples from the Tomoka River basin had greater concentrations than the previously reported maximum for that basin. Total nitrogen concentrations for all of the samples from the B-19 Canal basin and for 75 percent of the samples from the Tomoka River basin exceeded $0.6 \mathrm{mg} / \mathrm{L}$, the suggested limit to prevent enormous amounts of plant growth (McKee and Wolf, 1963).

Dissolved-solids concentrations in 16 of 41 samples from the Tomoka River basin and 9 of 13 samples from the B-19 Canal basin were outside the previously reported ranges for these basins. The median dissolved-solids concentration of the samples collected was $114 \mathrm{mg} / \mathrm{L}$ for the Tomoka River basin and $204 \mathrm{mg} / \mathrm{L}$ for the B-19 Canal basin. Concentrations ranged from a low of $46 \mathrm{mg} / \mathrm{L}$ in the Tomoka River basin to a high of $458 \mathrm{mg} / \mathrm{L}$ in the B-19 Canal basin. All samples collected had concentrations well below the $1,000 \mathrm{mg} / \mathrm{L}$ criteria set for a potable water supply (Florida Department of Environmental Regulation, 1983).

Specific-conductance values for samples collected during this study ranged from less than 50 to more than $650 \mu \mathrm{S} / \mathrm{cm}$ (microsiemens per centimeter). Three samples from the B-19 Canal had concentrations that exceeded the $500 \mu \mathrm{S} / \mathrm{cm}$ limit set by the Florida Department of
Environmental Regulation (1983) for a potable water supply. The median value for the B-19 Canal was 293 $\mu \mathrm{S} / \mathrm{cm}$ compared to $121 \mu \mathrm{S} / \mathrm{cm}$ for the Tomoka River basin. The range of all mean daily conductance data from the monitors was 70 to $865 \mu \mathrm{S} / \mathrm{cm}$ (appendix VI). Mean daily conductance exceeded $500 \mu \mathrm{S} / \mathrm{cm}$ at sites 3 and 12 during the study period.

Chloride concentrations in the study area ranged from 3.4 to $57 \mathrm{mg} / \mathrm{L}$. All sample concentrations were well below the $250 \mathrm{mg} / \mathrm{L}$ criteria for a potable water supply (Florida Department of Environmental Regulation, 1983). Of the 54 samples collected, 4 samples had chloride concentrations greater than the previous maximum of $36 \mathrm{mg} / \mathrm{L}$, and 39 samples had concentrations lower than the previous minimum of $18 \mathrm{mg} / \mathrm{L}$.

Color of water in both basins generally was greater than the recommended limit of $45 \mathrm{Pt}-\mathrm{Co}$ (platinumcobalt) units based on photosynthesis interference (U.S. Environmental Protection Agency, 1986). Of the samples collected during this study, 100 percent of the B-19 Canal samples and 84 percent of the Tomoka River samples exceeded this criterion. The maximum color observed was $360 \mathrm{Pt}-\mathrm{Co}$ units in the Tomoka River basin and $340 \mathrm{Pt}-\mathrm{Co}$ units in the B-19 Canal.

Table 3. Summary of selected constituent limits and data from samples collected at surface-water sites

IUnits are milligrams per liter, except where indicated; < indicates less than; - indicates no data; $\mu \mathrm{S} / \mathrm{cm}$, microsiemens per centimeter; ${ }^{\circ} \mathrm{C}$, degree Celsius; Pt-Co, Platinum-Cobalt]

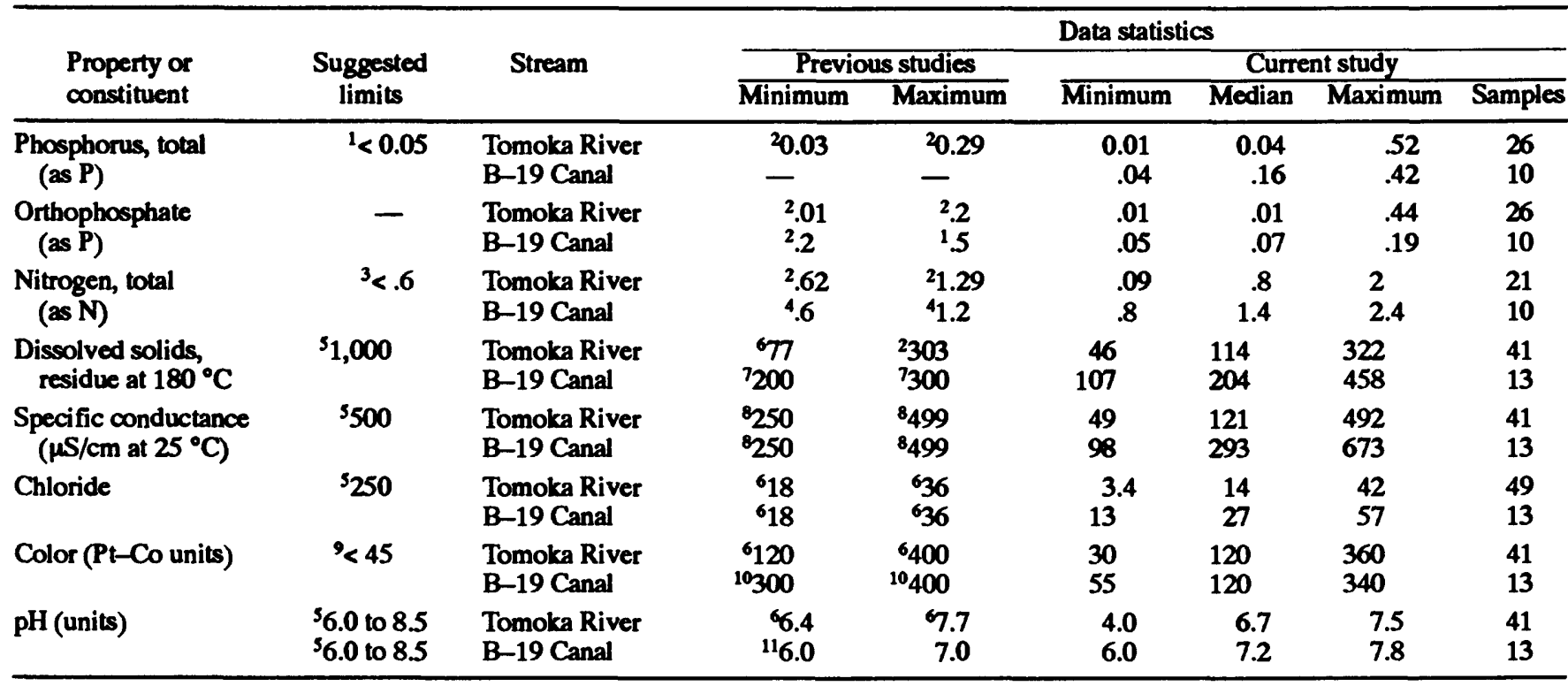

${ }^{1}$ Federal Water Pollution Control Administration, 1968.

2Volusia County Planning Board, 1978.

McKee and Wolf, 1963.

4 Slack and Goolsby, 1976.

${ }^{5}$ Florida Department of Environmental Regulation, 1983.
Knochenmus and Beard, 1971.

7 Dysart and Goolsby, 1977.

8 Slack and Kaufman, 1975.

'U.S. Environmental Protection Agency, 1986.

10Kaufman, 1975 .

${ }^{1}$ Kaufman, 1975c. 
The Tomoka River and B-19 Canal generally had pH values within the 6.0 to 8.5 range specified by the Florida Department of Environmental Regulation (1983) for class III waters. The only significant deviations were noted at site 5 (Thayer Canal near Daytona Beach which was consistently low), and site 8 (U.S. Highway 92 Canal at Daytona Beach which was generally low). These canals drain swampy areas with abundant decaying organic matter that produce tannic-acid-colored water with a pH in the 4.0 to 4.6 range, (appendix IV). The maximum observed $\mathrm{pH}$ during the study was 7.8 in the B-19 Canal.

The DO content of surface water gives a general indication of its ability to support aquatic life. The daily mean DO concentrations ranged from 3.5 to $9.1 \mathrm{mg} / \mathrm{L}$ at the Tomoka River near Holly Hill (fig. 2, site 1), and from 3.7 to $8.6 \mathrm{mg} / \mathrm{L}$ in the B-19 Canal at Dunlawton Avenue (fig. 2 , site 12). Within the two basins, the DO concentration commonly fluctuated between 4 and $6 \mathrm{mg} / \mathrm{L}$ with many values below the $5.0 \mathrm{mg} / \mathrm{L}$ minimum criterion for freshwater, and occasionally below the $4.0 \mathrm{mg} / \mathrm{L}$ absolute minimum criterion set by Florida Department of Environmental Regulation (1983) for class III waters. Appendix VII presents the DO data for the two streams.

Water samples and bed-material samples were collected near the most downstream sites on the two major streams (sites 2 and 12) and analyzed for insecticides and herbicides. Only three organic insecticides, diazinon, DDD, and heptachlor, and one herbicide, 2,4-D, were detected, and all were found at both sites. Diazinon concentrations in the water were $0.01 \mu \mathrm{g} / \mathrm{L}$ (micrograms per liter) in the Tomoka River basin (site 2) and $0.23 \mu \mathrm{g} / \mathrm{L}$ in the B-19 Canal basin (site 12) (appendix IV). The 2,4-D concentrations in the water were $<0.01 \mu \mathrm{g} / \mathrm{L}$ at site 2 and $0.09 \mu \mathrm{g} / \mathrm{L}$ at site 12 . In the bottom material, DDD concentrations were $0.3 \mu \mathrm{g} / \mathrm{kg}$ (micrograms per kilogram) at site 2 and $<0.1 \mu \mathrm{g} / \mathrm{kg}$ at site 12 . Heptachlor concentrations were $0.2 \mu \mathrm{g} / \mathrm{kg}$ at site 2 and $<0.01 \mu \mathrm{g} / \mathrm{kg}$ at site 12 .

No criteria have been established for diazinon or DDD, and the $100 \mu \mathrm{g} / \mathrm{L}$ limit for 2,4-D is for water and fish ingestion set by the U.S. Environmental Protection Agency (1986). The criterion for heptachlor, $0.52 \mu \mathrm{g} / \mathrm{L}$, is for heptachlor in solution, and should not be compared with bed-sediment concentrations detected (micrograms per kilogram, solids). Because limits do not exist, or are for a different medium, no conclusions were drawn from the detected concentrations of these pesticides.

Water analyses were also run for aluminum, arsenic copper, iron, lead, mercury, and zinc during the current study (appendix IV). Aluminum concentrations generally were in the few hundreds of micrograms per liter, although concentrations ranged from 30 to $8,200 \mu \mathrm{g} / \mathrm{L}$ in the Tomoka River basin, and from 30 to $4,100 \mu \mathrm{g} / \mathrm{L}$ in the B-19 Canal. The only criterion for aluminum is for marine waters; of the samples collected, three had concentrations exceeding the $1,500 \mu \mathrm{g} / \mathrm{L}$ limit set by the Florida Department of Environmental Regulation (1983) for marine waters.

None of the samples collected (appendix IV) had concentrations that exceeded FDER limits set for class III waters for copper $(30 \mu \mathrm{g} / \mathrm{L})$, iron $(1.0 \mathrm{mg} / \mathrm{L})$, and lead $(30 \mu \mathrm{g} / \mathrm{L})$ (Florida Department of Environmental Regulation, 1983). No sample concentrations exceeded the arsenic limit $(50 \mu \mathrm{g} / \mathrm{L})$ set by the U.S. Environmental Protection Agency (1986) for a potable water supply.

Three samples (15 percent) from the Tomoka River basin had concentrations of mercury greater than the $\mathbf{0 . 2}$ $\mu \mathrm{g} / \mathrm{L}$ criteria set by the Florida Department of Environmental Regulation (1983) for class III waters, whereas 2, or 25 percent, of the B-19 Canal samples exceeded the $0.2 \mu \mathrm{g} / \mathrm{L}$ criteria.

No samples from the B-19 Canal had zinc concentrations that exceeded the $30-\mu \mathrm{g} / \mathrm{L}$ limit set by the Florida Department of Environmental Regulation (1983) for class III waters. Twenty-five percent of the zinc concentrations for samples collected from the Tomoka River basin were greater than the $30-\mu \mathrm{g} / \mathrm{L}$ standard.

Analyses for alkalinity (appendix IV) showed that 39 percent of the samples from the Tomoka River basin and 23 percent of the samples from the B-19 Canal basin were below the minimum $20 \mathrm{mg} / \mathrm{L}$ set by Florida Department of Environmental Regulation (1983) for class III freshwater.

The quality of water in the Tomoka River basin in October 1985 generally was within the FDER criteria for class III waters, about the same findings as reported by the Volusia County Planning Board in 1978. The water in the B-19 Canal tends to have higher concentrations of total phosphorus, total nitrogen, and dissolved solids, and higher specific-conductance values than does water in the Tomoka River. Two tributaries to the Tomoka River, however, had higher color and lower $\mathrm{pH}$.

\section{LOADS FOR SELECTED CONSTITUENTS}

Constituent load is the amount, by weight, of a constituent transported within the streamflow during some time period. Commonly, this rate is expressed in pounds per day; but for this report, load is expressed as the total pounds of a constituent that flowed past a particular point (the gaging site) during the selected storm period. Loads for phosphorus, nitrogen, and dissolved solids were calculated for selected storms at nine surface-water sites (table 4). 
Table 4. Phosphorus, nitrogen, and dissolved-solids loads for selected storms in the Tomoka River and B-19 Canal basins $\left[\mathrm{mi}^{2}\right.$, square miles; $\mathrm{ft}^{3}$, cubic feet; $\mathrm{lb}$, pounds; $\mathrm{lb} / \mathrm{mi}^{2}$, pounds per square mile; - , indicates no data]

\begin{tabular}{|c|c|c|c|c|c|c|c|c|c|c|}
\hline \multirow{3}{*}{$\begin{array}{l}\text { Site } \\
\text { No. }\end{array}$} & \multirow{3}{*}{ Station name } & \multirow{3}{*}{$\begin{array}{c}\text { Drain- } \\
\text { age } \\
\text { area } \\
\left(\mathrm{mi}^{2}\right)\end{array}$} & \multirow{3}{*}{$\begin{array}{l}\text { Storm } \\
\text { date }\end{array}$} & \multirow{3}{*}{$\begin{array}{l}\text { Storm } \\
\text { runoff } \\
\left.\text { (million } \mathrm{f}^{3}\right)\end{array}$} & \multicolumn{6}{|c|}{ Storm loads } \\
\hline & & & & & \multicolumn{2}{|c|}{ Phosphorus } & \multicolumn{2}{|c|}{ Nitrogen } & \multicolumn{2}{|c|}{ Dissolved solids } \\
\hline & & & & & (lb) & $\left(\mathrm{lb} / \mathrm{mi}^{2}\right)$ & (lb) & $\left(\mathrm{lb} / \mathrm{mi}^{2}\right)$ & (tons) & (tons $\left./ \mathrm{mi}^{2}\right)$ \\
\hline \multicolumn{11}{|c|}{ Tomoka River basin } \\
\hline 1 & $\begin{array}{l}\text { Tomoka River near } \\
\text { Holly Hill }\end{array}$ & 63 & $09-28-84$ & 164 & 824 & 13.1 & 8,240 & 131 & 393.5 & 6.2 \\
\hline 2 & $\begin{array}{l}\text { Eleventh Street Canal } \\
\text { near Holly Hill }\end{array}$ & 3.5 & $\begin{array}{l}12-12-83 \\
09-28-84\end{array}$ & $\begin{array}{l}5.70 \\
9.65\end{array}$ & $\overline{66.4}$ & $\overline{19.0}$ & $\overline{664}$ & $\overline{190}$ & $\begin{array}{l}23.2 \\
20.5\end{array}$ & $\begin{array}{l}6.6 \\
5.8\end{array}$ \\
\hline 3 & $\begin{array}{l}\text { Williamson Boulevard } \\
\text { Ditch at Daytona } \\
\text { Beach }\end{array}$ & .3 & $\begin{array}{l}04-04-84 \\
05-23-84\end{array}$ & $\begin{array}{l}2.20 \\
1.38\end{array}$ & $\begin{array}{l}4.12 \\
5.17\end{array}$ & $\begin{array}{l}13.7 \\
17.2\end{array}$ & $\begin{array}{c}151 \\
17.2\end{array}$ & $\begin{array}{l}503 \\
57.3\end{array}$ & $\begin{array}{l}3.2 \\
2.4\end{array}$ & $\begin{array}{r}10.8 \\
8.0\end{array}$ \\
\hline 4 & $\begin{array}{l}\text { Wally Hoffmeyer } \\
\text { Canal at Daytona } \\
\text { Beach }\end{array}$ & 2.2 & $12-21-83$ & 8.67 & - & - & - & - & 31.4 & 14.3 \\
\hline 5 & $\begin{array}{l}\text { Thayer Canal near } \\
\text { Daytona Beach }\end{array}$ & 33 & $09-28-84$ & 26.2 & 16.4 & 0.5 & 1,163 & 35.2 & - & - \\
\hline 6 & $\begin{array}{l}\text { Bayless Boulevard } \\
\text { Canal at Daytona } \\
\text { Beach }\end{array}$ & 1.4 & $12-21-83$ & 5.45 & - & - & - & - & 17.0 & 12.2 \\
\hline 7 & $\begin{array}{l}\text { Bellevue Canal at } \\
\text { Daytona Beach }\end{array}$ & 1.5 & $04-04-84$ & 7.94 & 14.9 & 9.9 & 546 & 364 & 22.4 & 14.9 \\
\hline \multicolumn{11}{|c|}{$\underline{\text { B-19 Canal basin }}$} \\
\hline 11 & $\begin{array}{l}\text { B-19 Canal at } \\
\text { Willow Run Boulevard } \\
\text { at Port Orange }\end{array}$ & 5.8 & $04-04-84$ & 7.44 & 97.4 & 16.8 & 974 & 168 & 29.5 & 5.1 \\
\hline 12 & $\begin{array}{l}\text { B-19 Canal at } \\
\text { Dunlawton Avenue } \\
\text { at Port Orange }\end{array}$ & 7.6 & $04-04-84$ & 24.1 & 286 & 37.6 & 3,310 & 436 & 80.5 & 10.6 \\
\hline
\end{tabular}

The equation for calculating loads is:

$$
\mathrm{L}=\mathrm{K} * \mathrm{C} * \mathrm{~V}
$$

where

$L=$ load for storm, in pounds,

$\mathrm{K}=$ conversion factor of $6.24 \times 10^{-5}$ for converting between milligram per liter and pound per cubic foot,

C = concentration of the constituent, in milligrams per liter, and

$V$ = total runoff volume for the storm, in cubic feet.

To compare loads from drainage basins of different sizes more easily, load values are expressed in table 4 as yields, in pounds per square mile of drainage area. Total phosphorus yields ranged from 0.5 to $19 \mathrm{lb} / \mathrm{mi}^{2}$ (pounds per square mile) in the Tomoka River basin, and from 16.8 to $37.6 \mathrm{lb} / \mathrm{mi}^{2}$ in the B-19 Canal basin. Total nitrogen yields ranged from 35.2 to $503 \mathrm{lb} / \mathrm{mi}^{2}$ in the Tomoka River basin, and from 168 to $436 \mathrm{lb} / \mathrm{mi}^{2}$ in the B-19 Canal basin. Dissolved-solids yields ranged from 5.8 to 14.9 tons $/ \mathrm{mi}^{2}$ (tons per square mile) within the Tomoka River basin, and from 5.1 to 10.6 tons $/ \mathrm{mi}^{2}$ in the B-19 Canal basin.

\section{SUMMARY}

Rainfall, streamflow, and water-quality data were collected from the Tomoka River and the B-19 Canal basins from October 1982 through September 1985 to better understand the quantity and quality of stormwater runoff from western Daytona Beach and adjacent areas. Data were collected at 12 surface-water sites and 8 rainfall sites. Stagedischarge ratings were developed for eight sites; a ninth site already had an established rating. At three sites, hydraulic conditions were unsatisfactory for developing a rating. 
Hydrographs plotted for selected storms at each of the nine rated sites were used to compute storm runoff. Runoff for the Tomoka River ranged from 1 to 77 percent of basin rainfall. For the B-19 Canal, runoff ranged from 6 to 17 percent of rainfall. Ratios of runoff to rainfall were widely varied, even for storms occurring at the same sites; few patterns were discernible from the rainfall-runoff plots. Poor relations between runoff and rainfall probably are due to (1) interconnecting canals that allow flow to move through the basins by several routes, (2) backwater conditions that resulted in reversed flow, and (3) storage in large marshy areas that retarded runoff.

Total phosphorus concentrations for the samples collected within the two basins ranged from 0.01 to $0.52 \mathrm{mg} / \mathrm{L}$. The highest concentration occurred in the Tomoka River basin, but the B-19 Canal basin had the largest median value, $0.16 \mathrm{mg} / \mathrm{L}$. Thirty-three percent of the samples from the Tomoka River basin and 90 percent from the B-19 Canal basin had total phosphorus concentrations exceeding $0.05 \mathrm{mg} / \mathrm{L}$. Median orthophosphate concentrations were 0.01 and $0.07 \mathrm{mg} / \mathrm{L}$ for the Tomoka River basin and the B-19 Canal basin, respectively.

Total nitrogen concentrations for the samples collected ranged from 0.09 to $2.4 \mathrm{mg} / \mathrm{L}$. All of the samples collected in the B-19 Canal basin and 75 percent from the Tomoka River basin exceeded $0.6 \mathrm{mg} / \mathrm{L}$.

The maximum dissolved-solids concentration observed during the study was $458 \mathrm{mg} / \mathrm{L}$ in a sample from the B-19 Canal basin. The range for all samples was 46 to $458 \mathrm{mg} / \mathrm{L}$, well below the $1,000 \mathrm{mg} / \mathrm{L}$ dissolved solids set by the FDER as the criterion for a potable water supply.

Specific-conductance values ranged from 49 to 865 $\mu \mathrm{S} / \mathrm{cm}$, the latter being a mean daily value recorded by monitoring. Mean daily conductance exceeded the 500 $\mu \mathrm{S} / \mathrm{cm}$ limit at one site in each basin during the study period. Chloride concentrations for all samples ranged from 3.4 to $57 \mathrm{mg} / \mathrm{L}$. These values are below the $250 \mathrm{mg} / \mathrm{L}$ criterion set by the FDER for a potable water supply.

The maximum color observed in samples from the study area was $360 \mathrm{Pt}-\mathrm{Co}$ units in the Tomoka River basin. All samples from the B-19 Canal basin exceeded the 45 $\mathrm{Pt}-\mathrm{Co}$ unit criterion set by the USEPA, whereas 84 percent of the samples from the Tomoka River basin exceeded this limit. All but two of the sites in the study area had $\mathrm{pH}$ values within the FDER specified range for class III waters, 6.0 to $8.5 \mathrm{pH}$ units. Thayer Canal and U.S. Highway 92 Canal, which drain swampy areas, had water with $\mathrm{pH}$ as low as 4.0.

Dissolved-oxygen concentrations at Tomoka River near Holly Hill, the most downstream site in the Tomoka River basin, ranged from 3.5 to $9.1 \mathrm{mg} / \mathrm{L}$. Dissolvedoxygen concentrations for the B-19 Canal at Dunlawton Avenue, the most downstream site in the B-19 Canal basin, ranged from 3.7 to $8.6 \mathrm{mg} / \mathrm{L}$. Frequently, the dissolved-oxygen concentrations were below the $5.0-\mathrm{mg} / \mathrm{L}$ criterion set by the FDER as the minimum daily average. Concentrations between 4.0 and 6.0 were common.

Analysis of water and bed-material samples near each of the most downstream sites detected the insecticides diazinon, DDD, and heptachlor and the herbicide 2,4-D. Diazinon concentrations in water were $0.01 \mu \mathrm{g} / \mathrm{L}$ in the Tomoka River basin and $0.23 \mu \mathrm{g} / \mathrm{L}$ in the B-19 Canal basin, and 2,4-D concentrations in water were $<0.01 \mu \mathrm{g} / \mathrm{L}$ in the Tomoka River basin and $0.09 \mu \mathrm{g} / \mathrm{L}$ in the B-19 Canal basin. DDD concentrations of $0.3 \mu \mathrm{g} / \mathrm{kg}$ and $<0.1 \mu \mathrm{g} / \mathrm{kg}$ and heptachlor concentrations of $0.2 \mu \mathrm{g} / \mathrm{kg}$ and $<0.1 \mu \mathrm{g} / \mathrm{kg}$ were detected in bottom sediments in the Tomoka River basin and B-19 Canal basin, respectively.

Analyses of water were also run for aluminum, arsenic, copper, iron, lead, mercury, and zinc. Of these, mercury and zinc were the only constituents with concentrations that exceeded the recommended limits for FDER class III waters. A total of five samples from both basins exceeded the 0.2$\mu \mathrm{g} / \mathrm{L}$ mercury criterion for a potable water supply. Twentyfive percent of the Tomoka River samples and none from the B-19 Canal exceeded the 30- $\mu \mathrm{g} / \mathrm{L}$ limit for zinc.

Loads (yields) of total phosphorus, total nitrogen, and dissolved solids were calculated for selected storms at rated sites. Total phosphorus yields ranged from 0.5 to 37.6 $\mathrm{lb} / \mathrm{mi}^{2}$, with the higher yield occurring in the B-19 Canal basin. Total nitrogen yields ranged from 35.2 to $503 \mathrm{lb} / \mathrm{mi}^{2}$; the highest yield was in the Tomoka River basin. Dissolvedsolids yields ranged between 5.1 and 14.9 tons $/ \mathrm{mi}^{2}$, with the highest yield being in the Tomoka River basin.

\section{SELECTED REFERENCES}

Baskerville-Donovan Engineers, Inc., 1985, B-19 Canal upper basin study: Volusia County, $100 \mathrm{p}$.

Bush, P.W., 1978, Hydrologic evaluation of part of central Volusia County, Florida: U.S. Geological Survey Water-Resources Investigation 78-89, $50 \mathrm{p}$.

Dysart, J.E., and Goolsby, D.A., 1977, Dissolved solids concentrations and loads in Florida surface waters: Florida Bureau of Geology Map Series 77, 1 sheet.

Florida Department of Environmental Regulation, 1983, Water-quality standards: chap. 17-3, in Florida Administrative Code.

Federal Water Pollution Control Administration, 1968, Water-quality criteria-Report of the National Technical Advisory Committee to the Secretary of the Interior, $234 \mathrm{p}$.

Gilman, C.S., 1964, Section 9, Rainfall, in Chow, V.T., ed., Handbook of applied hydrology: New York, McGraw-Hill, $68 \mathrm{p}$.

Hardee, Jack, Miller, R.A., and Mattraw, H.C., Jr., 1979, Stormwater-runoff data for a multifamily residential area, Dade County, Florida: U.S. Geological Survey Open-File Report 79-1295, 68 p. 
Hem, J.D., 1985, Study and interpretation of the chemical characteristics of natural water ( $3 d$ ed.): U.S. Geological Survey Water-Supply Paper 2254, 263 p.

Hughes, G.H., 1978, Runoff from hydrologic units in Florida: Florida Bureau of Geology Map Series 81, 1 sheet.

Kaufman, M.I., 1975a, Color of water in Florida streams and canals (2d ed.): Florida Bureau of Geology Map Series 35, 1 sheet.

$1975 \mathrm{~b}$, Generalized distribution and concentration of orthophosphate in Florida streams (2d ed.): Florida Bureau of Geology Map Series 33, 1 sheet.

1975c, The chemical type of water in Florida streams (2d ed.): Florida Bureau of Geology Map Series 51, 1 sheet.

1975d, The $\mathrm{pH}$ of water in Florida streams and canals (2d ed.): Florida Bureau of Geology Map Series 37, 1 sheet.

Knochenmus, D.D., 1968, Surface drainage characteristics in Volusia County, Florida: Florida Division of Geology Map Series 30, 1 sheet.

Knochenmus, D.D., and Beard, M.E., 1971, Evaluation of the quantity and quality of the water resources of Volusia County, Florida: Florida Bureau of Geology Report of Investigations 57,59 p.
McKee, J.E., and Wolf, H.W., 1963, Water quality criteria: State Water Quality Control Board, pub. 3A, Sacramento, Calif., $548 \mathrm{p}$.

Rutledge, A.T., 1985, Ground-water hydrology of Volusia County, Forida, with emphasis on occurrence and movement of brackish water: U.S. Geological Survey Water-Resources Investigations Report 84-4206, 84 p.

Slack, L.J., and Goolsby, D.A., 1976, Nitrogen loads and concentrations in Florida streams: Florida Bureau of Geology Map Series 75, 1 sheet.

Slack, L.J., and Kaufman, M.I., 1975, Specific conductance of water in Florida streams and canals (2d ed.): Florida Bureau of Geology Map Series 58, 1 sheet.

U.S. Environmental Protection Agency, 1986, Quality criteria for water, 1986: Office of Water Regulations and Standards, EPA 440/5-86-001, update 1.0, 2 p.

U.S. Soil Conservation Service, 1985, Flood plain management study, tributaries to the Tomoka River, Volusia County, Florida: Gainesville, Florida, U.S. Department of Agriculture, $81 \mathrm{p}$.

Volusia County Planning Board, 1978, Tomoka River watershed water quality study, in Volusia Council of Governments 208 Water Quality Management Plan, Volusia County, Florida, $150 \mathrm{p}$. 


\section{Appendix I}


APPERDIX I--Daily rainfall

[Rainfall in inches]

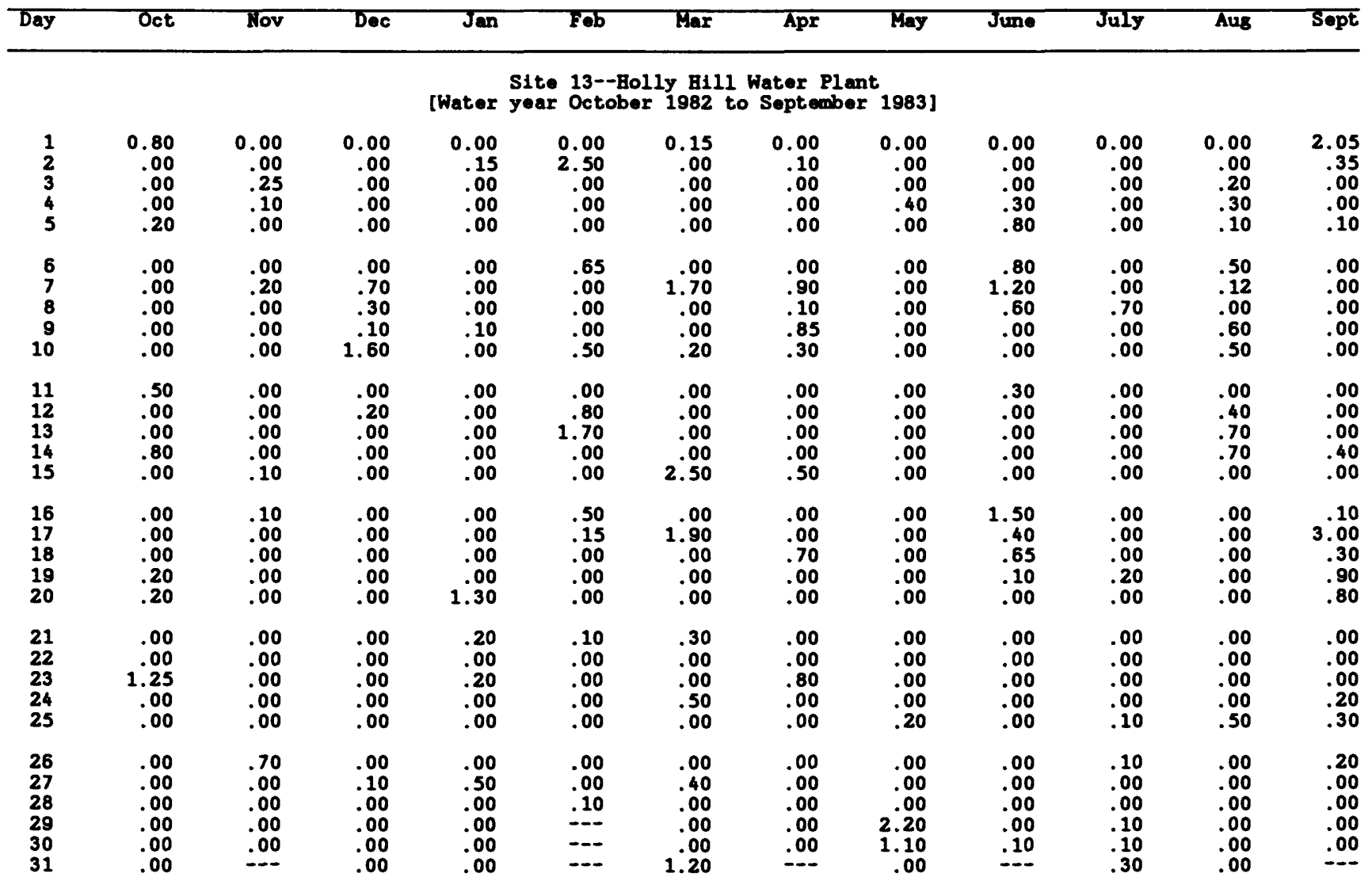

S1te 13--Eolly Bill Water Plant

[Water year October 1983 to September 1984]

\begin{tabular}{|c|c|c|c|c|c|c|c|c|c|c|c|c|}
\hline $\begin{array}{l}1 \\
2 \\
3 \\
4 \\
5\end{array}$ & $\begin{array}{r}0.00 \\
.00 \\
.00 \\
.00 \\
.00\end{array}$ & $\begin{array}{r}0.00 \\
.00 \\
.00 \\
.00 \\
.16\end{array}$ & $\begin{array}{r}0.00 \\
.00 \\
.00 \\
.20 \\
.00\end{array}$ & $\begin{array}{r}0.00 \\
.00 \\
.00 \\
.00 \\
.00\end{array}$ & $\begin{array}{r}0.00 \\
.10 \\
.20 \\
.00 \\
.00\end{array}$ & $\begin{array}{r}0.00 \\
.00 \\
.00 \\
.00 \\
.00\end{array}$ & $\begin{array}{r}0.00 \\
.00 \\
4.50 \\
1.30 \\
.00\end{array}$ & $\begin{array}{r}0.00 \\
.00 \\
.00 \\
1.80 \\
.00\end{array}$ & $\begin{array}{r}0.00 \\
.00 \\
.00 \\
.00 \\
.00\end{array}$ & $\begin{array}{r}1.30 \\
1.10 \\
1.00 \\
.00 \\
.00\end{array}$ & $\begin{array}{r}0.00 \\
.00 \\
.00 \\
.00 \\
.00\end{array}$ & $\begin{array}{r}0.00 \\
.00 \\
.00 \\
.30 \\
1.00\end{array}$ \\
\hline $\begin{array}{r}6 \\
7 \\
8 \\
9 \\
10\end{array}$ & $\begin{array}{l}.00 \\
.00 \\
.30 \\
.20 \\
.00\end{array}$ & $\begin{array}{l}.00 \\
.10 \\
.00 \\
.00 \\
.00\end{array}$ & $\begin{array}{l}.10 \\
.00 \\
.00 \\
.00 \\
.00\end{array}$ & $\begin{array}{l}.00 \\
.00 \\
.00 \\
.00 \\
.25\end{array}$ & $\begin{array}{l}.00 \\
.00 \\
.00 \\
.00 \\
.00\end{array}$ & $\begin{array}{l}.00 \\
.15 \\
.00 \\
.00 \\
.00\end{array}$ & $\begin{array}{l}.00 \\
.00 \\
.00 \\
.10 \\
.00\end{array}$ & $\begin{array}{l}.00 \\
.00 \\
.00 \\
.00 \\
.00\end{array}$ & $\begin{array}{l}.00 \\
.00 \\
.00 \\
.00 \\
.00\end{array}$ & $\begin{array}{l}.00 \\
.00 \\
.00 \\
.00 \\
.00\end{array}$ & $\begin{array}{l}.00 \\
.00 \\
.00 \\
.00 \\
.00\end{array}$ & $\begin{array}{r}1.50 \\
1.00 \\
.00 \\
4.00 \\
.10\end{array}$ \\
\hline $\begin{array}{l}11 \\
12 \\
13 \\
14 \\
15\end{array}$ & $\begin{array}{r}1.20 \\
.00 \\
.20 \\
.00 \\
.20\end{array}$ & $\begin{array}{l}.10 \\
.00 \\
.00 \\
.00 \\
.15\end{array}$ & $\begin{array}{r}3.80 \\
1.60 \\
.00 \\
.00 \\
.00\end{array}$ & $\begin{array}{l}.00 \\
.00 \\
.10 \\
.00 \\
.00\end{array}$ & $\begin{array}{l}.00 \\
.20 \\
.00 \\
.00 \\
.00\end{array}$ & $\begin{array}{l}.00 \\
.10 \\
.20 \\
.00 \\
.00\end{array}$ & $\begin{array}{l}.00 \\
.00 \\
.10 \\
.80 \\
.00\end{array}$ & $\begin{array}{l}.00 \\
.00 \\
.00 \\
.00 \\
.00\end{array}$ & $\begin{array}{l}.00 \\
.10 \\
.20 \\
.00 \\
.00\end{array}$ & $\begin{array}{l}.00 \\
.00 \\
.10 \\
.00 \\
.00\end{array}$ & $\begin{array}{l}.00 \\
.60 \\
.00 \\
.00 \\
.00\end{array}$ & $\begin{array}{l}.00 \\
.00 \\
.00 \\
.00 \\
.00\end{array}$ \\
\hline $\begin{array}{l}16 \\
17 \\
18 \\
19 \\
20\end{array}$ & $\begin{array}{r}1.20 \\
.75 \\
.00 \\
.10 \\
.00\end{array}$ & $\begin{array}{l}.25 \\
.00 \\
.00 \\
.00 \\
.45\end{array}$ & $\begin{array}{r}.10 \\
1.50 \\
.10 \\
.20 \\
3.20\end{array}$ & $\begin{array}{l}.00 \\
.00 \\
.20 \\
.50 \\
.50\end{array}$ & $\begin{array}{l}.00 \\
.00 \\
.00 \\
.00 \\
.00\end{array}$ & $\begin{array}{l}.00 \\
.00 \\
.20 \\
.00 \\
.60\end{array}$ & $\begin{array}{l}.00 \\
.00 \\
.00 \\
.00 \\
.00\end{array}$ & $\begin{array}{l}.00 \\
.00 \\
.00 \\
.00 \\
.00\end{array}$ & $\begin{array}{l}.00 \\
.00 \\
.00 \\
.00 \\
.00\end{array}$ & $\begin{array}{l}.00 \\
.00 \\
.00 \\
.70 \\
.20\end{array}$ & $\begin{array}{l}.00 \\
.30 \\
.10 \\
.90 \\
.70\end{array}$ & $\begin{array}{r}.00 \\
.00 \\
1.10 \\
.05 \\
.00\end{array}$ \\
\hline $\begin{array}{l}21 \\
22 \\
23 \\
24 \\
25\end{array}$ & $\begin{array}{r}.10 \\
2.26 \\
.10 \\
.00 \\
.00\end{array}$ & $\begin{array}{l}.00 \\
.00 \\
.00 \\
.20 \\
.00\end{array}$ & $\begin{array}{l}.40 \\
.05 \\
.00 \\
.00 \\
.00\end{array}$ & $\begin{array}{l}.00 \\
.00 \\
.10 \\
.00 \\
.25\end{array}$ & $\begin{array}{r}.50 \\
1.00 \\
.05 \\
.00 \\
.00\end{array}$ & $\begin{array}{l}.00 \\
.00 \\
.00 \\
.10 \\
.00\end{array}$ & $\begin{array}{l}.00 \\
.00 \\
.10 \\
.00 \\
.00\end{array}$ & $\begin{array}{r}.00 \\
.10 \\
3.90 \\
.10 \\
.00\end{array}$ & $\begin{array}{l}.00 \\
.30 \\
.60 \\
.00 \\
.10\end{array}$ & $\begin{array}{l}.80 \\
.80 \\
.10 \\
.00 \\
.00\end{array}$ & $\begin{array}{l}.40 \\
.00 \\
.00 \\
.10 \\
.70\end{array}$ & $\begin{array}{l}.00 \\
.00 \\
.00 \\
.00 \\
.00\end{array}$ \\
\hline $\begin{array}{l}26 \\
27 \\
28 \\
29 \\
30 \\
31\end{array}$ & $\begin{array}{l}.00 \\
.00 \\
.00 \\
.00 \\
.00 \\
.20\end{array}$ & $\begin{array}{l}.00 \\
.00 \\
.00 \\
.70 \\
.00 \\
-.0\end{array}$ & $\begin{array}{r}.00 \\
.00 \\
.10 \\
2.40 \\
.00 \\
.00\end{array}$ & $\begin{array}{l}.10 \\
.00 \\
.00 \\
.00 \\
.00 \\
.00\end{array}$ & $\begin{array}{l}.00 \\
.60 \\
.00 \\
.00 \\
--- \\
---\end{array}$ & $\begin{array}{l}.10 \\
.50 \\
.00 \\
.00 \\
.00 \\
.00\end{array}$ & $\begin{array}{l}.00 \\
.00 \\
.00 \\
.00 \\
.00 \\
.0\end{array}$ & $\begin{array}{l}.80 \\
.00 \\
.00 \\
.00 \\
.10 \\
.00\end{array}$ & $\begin{array}{r}.00 \\
.00 \\
.00 \\
.50 \\
1.00 \\
\end{array}$ & $\begin{array}{l}.00 \\
.00 \\
.00 \\
.00 \\
.15 \\
.05\end{array}$ & $\begin{array}{l}.00 \\
.00 \\
.00 \\
.00 \\
.00 \\
.00\end{array}$ & $\begin{array}{r}.00 \\
2.80 \\
.10 \\
.00 \\
.00 \\
-\end{array}$ \\
\hline
\end{tabular}


APPENDIX I--Daily rainfal1--Continued [Rainfall in inches]

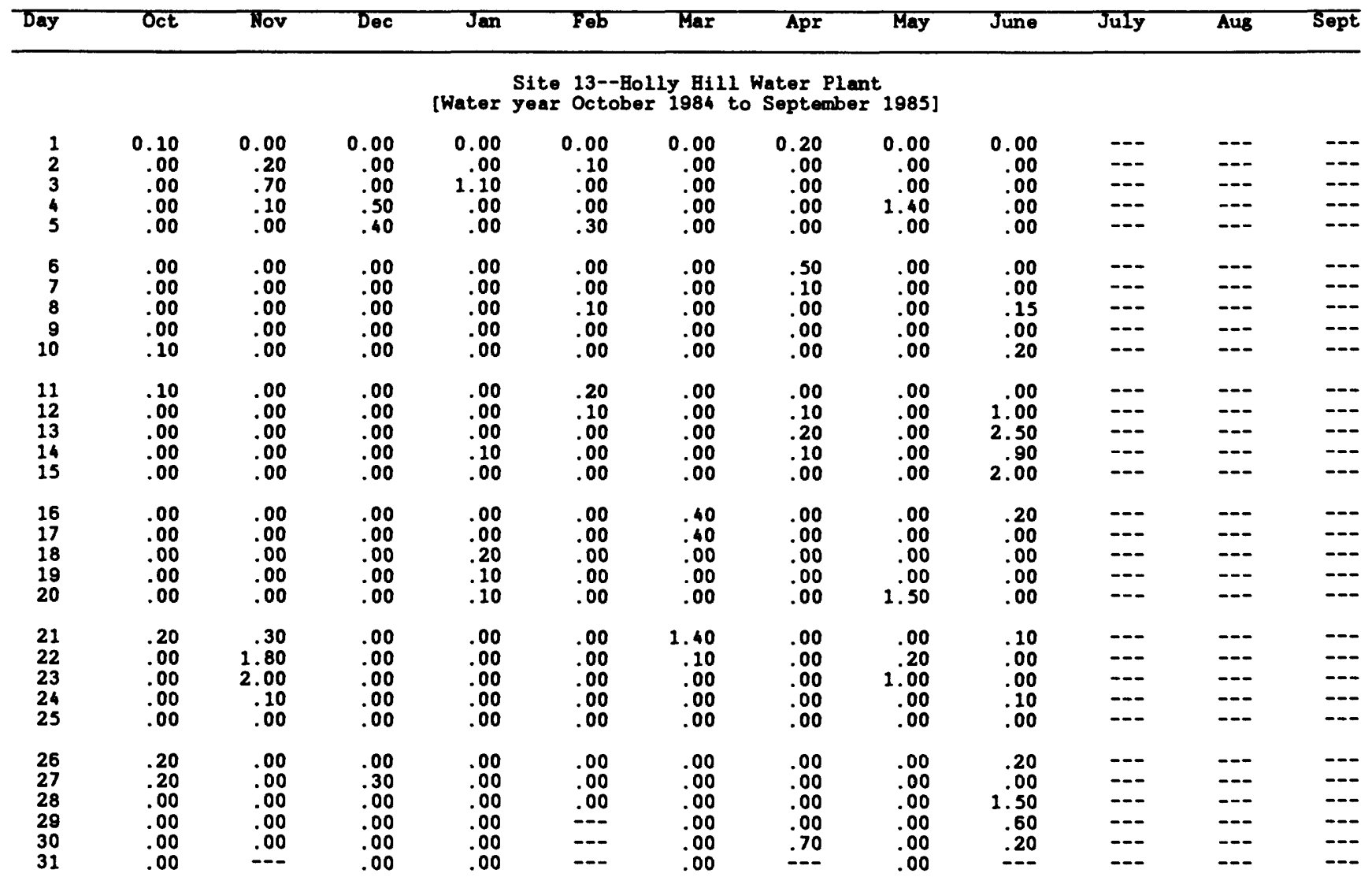

[Water year October 1982 to September 1983]

\begin{tabular}{|c|c|c|c|c|c|c|c|c|c|c|c|c|}
\hline $\begin{array}{l}1 \\
2 \\
3 \\
4 \\
5\end{array}$ & $\begin{array}{l}--- \\
--- \\
--- \\
---\end{array}$ & $\begin{array}{l}--- \\
--- \\
--- \\
--- \\
---\end{array}$ & $\begin{array}{l}--- \\
--- \\
--- \\
---\end{array}$ & $\begin{array}{l}--- \\
--- \\
--- \\
---\end{array}$ & $\begin{array}{l}--- \\
--- \\
--- \\
--- \\
--\end{array}$ & $\begin{array}{l}--- \\
--- \\
--- \\
---\end{array}$ & $\begin{array}{r}0.00 \\
.11 \\
.00 \\
.00 \\
.00\end{array}$ & $\begin{array}{r}0.00 \\
.00 \\
.00 \\
.37 \\
.02\end{array}$ & $\begin{array}{r}0.00 \\
.00 \\
.00 \\
.76 \\
.40\end{array}$ & $\begin{array}{r}0.05 \\
.02 \\
.00 \\
.00 \\
.28\end{array}$ & $\begin{array}{r}0.00 \\
.03 \\
.00 \\
.03 \\
.00\end{array}$ & $\begin{array}{r}1.63 \\
.40 \\
.65 \\
.00 \\
.00\end{array}$ \\
\hline $\begin{array}{r}6 \\
7 \\
8 \\
9 \\
10\end{array}$ & $\begin{array}{l}--- \\
-\cdots \\
---\end{array}$ & $\begin{array}{l}--- \\
--- \\
--- \\
--- \\
---\end{array}$ & $\begin{array}{l}--- \\
--- \\
--- \\
--- \\
--\end{array}$ & $\begin{array}{l}--- \\
--- \\
--- \\
--- \\
--\end{array}$ & $\begin{array}{l}--- \\
--- \\
--- \\
---\end{array}$ & $\begin{array}{l}--- \\
--- \\
--- \\
-00\end{array}$ & $\begin{array}{l}.00 \\
.21 \\
.00 \\
.97 \\
.07\end{array}$ & $\begin{array}{l}.00 \\
.00 \\
.00 \\
.10 \\
.00\end{array}$ & $\begin{array}{r}.79 \\
1.22 \\
.24 \\
.00 \\
.02\end{array}$ & $\begin{array}{l}.00 \\
.03 \\
.32 \\
.00 \\
.00\end{array}$ & $\begin{array}{r}.16 \\
.44 \\
.10 \\
1.83 \\
.36\end{array}$ & $\begin{array}{l}.00 \\
.00 \\
.00 \\
.00 \\
.00\end{array}$ \\
\hline $\begin{array}{l}11 \\
12 \\
13 \\
14 \\
15\end{array}$ & $\begin{array}{l}--- \\
-- \\
-- \\
--\end{array}$ & $\begin{array}{l}--- \\
--- \\
--- \\
---\end{array}$ & $\begin{array}{l}--- \\
--- \\
--- \\
---\end{array}$ & $\begin{array}{l}--- \\
--- \\
--- \\
--- \\
---\end{array}$ & $\begin{array}{l}--- \\
--- \\
--- \\
---\end{array}$ & $\begin{array}{r}.00 \\
.00 \\
.00 \\
.00 \\
2.06\end{array}$ & $\begin{array}{l}.00 \\
.00 \\
.35 \\
.00 \\
.89\end{array}$ & $\begin{array}{l}.07 \\
.00 \\
.00 \\
.00 \\
.00\end{array}$ & $\begin{array}{l}.01 \\
.00 \\
.06 \\
.00 \\
.00\end{array}$ & $\begin{array}{l}.02 \\
.01 \\
.00 \\
.07 \\
.00\end{array}$ & $\begin{array}{r}.00 \\
.56 \\
1.19 \\
.63 \\
.02\end{array}$ & $\begin{array}{l}.00 \\
.00 \\
.00 \\
.11 \\
.01\end{array}$ \\
\hline $\begin{array}{l}16 \\
17 \\
18 \\
19 \\
20\end{array}$ & $\begin{array}{l}--- \\
--- \\
--- \\
---\end{array}$ & $\begin{array}{l}--- \\
--- \\
--- \\
--- \\
---\end{array}$ & $\begin{array}{l}--- \\
--- \\
--- \\
---\end{array}$ & $\begin{array}{l}--- \\
--- \\
--- \\
--- \\
---\end{array}$ & $\begin{array}{l}--- \\
--- \\
--- \\
--- \\
---\end{array}$ & $\begin{array}{r}.00 \\
1.39 \\
.10 \\
.00 \\
.01\end{array}$ & $\begin{array}{l}.00 \\
.00 \\
.84 \\
.00 \\
.00\end{array}$ & $\begin{array}{l}.00 \\
.00 \\
.00 \\
.09 \\
.00\end{array}$ & $\begin{array}{l}.01 \\
.00 \\
.00 \\
.00 \\
.03\end{array}$ & $\begin{array}{l}.00 \\
.00 \\
.00 \\
.00 \\
.02\end{array}$ & $\begin{array}{l}.01 \\
.00 \\
.02 \\
.00 \\
.00\end{array}$ & $\begin{array}{l}.00 \\
.79 \\
.12 \\
.18 \\
.99\end{array}$ \\
\hline $\begin{array}{l}21 \\
22 \\
23 \\
24 \\
25\end{array}$ & $\begin{array}{l}--- \\
--- \\
--- \\
---\end{array}$ & $\begin{array}{l}--- \\
--- \\
--- \\
--- \\
---\end{array}$ & $\begin{array}{l}--- \\
--- \\
--- \\
--- \\
---\end{array}$ & $\begin{array}{l}--- \\
--- \\
--- \\
---\end{array}$ & $\begin{array}{l}--- \\
--- \\
--- \\
-- \\
---\end{array}$ & $\begin{array}{l}.51 \\
.00 \\
.00 \\
.49 \\
.00\end{array}$ & $\begin{array}{l}.00 \\
.00 \\
.98 \\
.00 \\
.00\end{array}$ & $\begin{array}{l}.00 \\
.00 \\
.00 \\
.00 \\
.17\end{array}$ & $\begin{array}{r}1.72 \\
.43 \\
.66 \\
.02 \\
.00\end{array}$ & $\begin{array}{l}.00 \\
.00 \\
.00 \\
.00 \\
.00\end{array}$ & $\begin{array}{l}.00 \\
.00 \\
.00 \\
.00 \\
.45\end{array}$ & $\begin{array}{l}.23 \\
.01 \\
.00 \\
.84 \\
.16\end{array}$ \\
\hline $\begin{array}{l}26 \\
27 \\
28 \\
29 \\
30 \\
31\end{array}$ & $\begin{array}{l}--- \\
--- \\
--- \\
--- \\
---\end{array}$ & $\begin{array}{l}--- \\
-- \\
--\overline{-} \\
--\overline{-} \\
--\overline{-} \\
--\end{array}$ & $\begin{array}{l}--- \\
--- \\
--- \\
--- \\
--- \\
---\end{array}$ & $\begin{array}{l}--- \\
--- \\
--- \\
--- \\
--- \\
---\end{array}$ & $\begin{array}{l}--- \\
--- \\
--- \\
--- \\
--- \\
---\end{array}$ & $\begin{array}{r}.00 \\
.44 \\
.01 \\
.00 \\
.02 \\
1.16\end{array}$ & $\begin{array}{l}.00 \\
.00 \\
.00 \\
.00 \\
.00 \\
.--\end{array}$ & $\begin{array}{r}.01 \\
.00 \\
.00 \\
1.39 \\
1.65 \\
.00\end{array}$ & $\begin{array}{l}.00 \\
.00 \\
.00 \\
.03 \\
.12 \\
-.--\end{array}$ & $\begin{array}{l}.42 \\
.01 \\
.05 \\
.76 \\
.10 \\
.68\end{array}$ & $\begin{array}{l}.00 \\
.00 \\
.27 \\
.03 \\
.00 \\
.00\end{array}$ & $\begin{array}{l}.12 \\
.00 \\
.00 \\
.00 \\
.00 \\
.0-\end{array}$ \\
\hline
\end{tabular}




\begin{tabular}{|c|c|c|c|c|c|c|c|c|c|c|c|c|}
\hline Day & Oct & Nov & Dec & $\operatorname{Jan}$ & Feb & Mar & Apr & May & June & July & Aug & Sept \\
\hline \multicolumn{13}{|c|}{$\begin{array}{l}\text { Site 14--Florida Power and Light } \\
\text { year October } 1983 \text { to September 1984] }\end{array}$} \\
\hline $\begin{array}{l}1 \\
2 \\
3 \\
4 \\
5\end{array}$ & $\begin{array}{l}0.00 \\
.00 \\
.00 \\
.00 \\
.00\end{array}$ & $\begin{array}{l}0.17 \\
.04 \\
.00 \\
.00 \\
.20\end{array}$ & $\begin{array}{l}0.00 \\
.00 \\
.00 \\
.01 \\
.00\end{array}$ & $\begin{array}{l}0.00 \\
.00 \\
.00 \\
.00 \\
.00\end{array}$ & $\begin{array}{l}0.01 \\
.01 \\
.00 \\
.00 \\
.00\end{array}$ & $\begin{array}{r}0.00 \\
.00 \\
.00 \\
.00 \\
.00\end{array}$ & $\begin{array}{r}0.00 \\
.00 \\
4.37 \\
1.81 \\
.00\end{array}$ & $\begin{array}{r}0.00 \\
.03 \\
.00 \\
2.25 \\
.01\end{array}$ & $\begin{array}{r}0.00 \\
.00 \\
.00 \\
.00 \\
.00\end{array}$ & $\begin{array}{r}1.93 \\
1.45 \\
1.07 \\
.00 \\
.23\end{array}$ & $\begin{array}{r}0.27 \\
.00 \\
.00 \\
.00 \\
.00\end{array}$ & $\begin{array}{r}0.00 \\
.00 \\
.00 \\
.78 \\
2.28\end{array}$ \\
\hline $\begin{array}{r}6 \\
7 \\
8 \\
9 \\
10\end{array}$ & $\begin{array}{r}.00 \\
.00 \\
1.14 \\
.62 \\
.31\end{array}$ & $\begin{array}{l}.01 \\
.05 \\
.00 \\
.18 \\
.00\end{array}$ & $\begin{array}{l}.05 \\
.00 \\
.00 \\
.00 \\
.00\end{array}$ & $\begin{array}{l}.00 \\
.00 \\
.02 \\
.00 \\
.23\end{array}$ & $\begin{array}{l}.00 \\
.00 \\
.00 \\
.00 \\
.00\end{array}$ & $\begin{array}{l}.00 \\
.06 \\
.00 \\
.00 \\
.00\end{array}$ & $\begin{array}{l}.00 \\
.00 \\
.00 \\
.26 \\
.00\end{array}$ & $\begin{array}{l}.00 \\
.00 \\
.00 \\
.01 \\
.00\end{array}$ & $\begin{array}{l}.00 \\
.00 \\
.00 \\
.00 \\
.00\end{array}$ & $\begin{array}{l}.00 \\
.05 \\
.00 \\
.00 \\
.00\end{array}$ & $\begin{array}{l}.00 \\
.00 \\
.00 \\
.00 \\
.00\end{array}$ & $\begin{array}{r}.99 \\
2.10 \\
.12 \\
3.16 \\
.27\end{array}$ \\
\hline $\begin{array}{l}11 \\
12 \\
13 \\
14 \\
15\end{array}$ & $\begin{array}{l}.72 \\
.01 \\
.13 \\
.01 \\
.72\end{array}$ & $\begin{array}{l}.10 \\
.00 \\
.02 \\
.00 \\
.26\end{array}$ & $\begin{array}{r}3.79 \\
1.95 \\
.00 \\
.01 \\
.21\end{array}$ & $\begin{array}{l}.00 \\
.00 \\
.13 \\
.03 \\
.00\end{array}$ & $\begin{array}{r}.00 \\
.49 \\
1.63 \\
.00 \\
.01\end{array}$ & $\begin{array}{l}.00 \\
.08 \\
.01 \\
.00 \\
.00\end{array}$ & $\begin{array}{r}.00 \\
.00 \\
.00 \\
1.25 \\
.02\end{array}$ & $\begin{array}{l}.00 \\
.00 \\
.00 \\
.00 \\
.00\end{array}$ & $\begin{array}{l}.26 \\
.00 \\
.17 \\
.00 \\
.00\end{array}$ & $\begin{array}{l}.00 \\
.00 \\
.19 \\
.00 \\
.00\end{array}$ & $\begin{array}{l}.00 \\
.18 \\
.00 \\
.00 \\
.00\end{array}$ & $\begin{array}{l}.00 \\
.00 \\
.00 \\
.00 \\
.00\end{array}$ \\
\hline $\begin{array}{l}16 \\
17 \\
18 \\
19 \\
20\end{array}$ & $\begin{array}{r}.94 \\
1.03 \\
.04 \\
.00 \\
.01\end{array}$ & $\begin{array}{l}.08 \\
.00 \\
.01 \\
.00 \\
.71\end{array}$ & $\begin{array}{r}.02 \\
.06 \\
.65 \\
.06 \\
3.10\end{array}$ & $\begin{array}{l}.00 \\
.01 \\
.06 \\
.47 \\
.50\end{array}$ & $\begin{array}{l}.00 \\
.00 \\
.00 \\
.00 \\
.00\end{array}$ & $\begin{array}{l}.00 \\
.00 \\
.11 \\
.00 \\
.52\end{array}$ & $\begin{array}{l}.00 \\
.06 \\
.00 \\
.00 \\
.00\end{array}$ & $\begin{array}{l}.00 \\
.00 \\
.00 \\
.00 \\
.00\end{array}$ & $\begin{array}{l}.00 \\
.00 \\
.56 \\
.00 \\
.00\end{array}$ & $\begin{array}{l}.00 \\
.01 \\
.00 \\
.50 \\
.63\end{array}$ & $\begin{array}{l}.00 \\
.02 \\
.07 \\
.30 \\
.78\end{array}$ & $\begin{array}{l}.00 \\
.05 \\
.72 \\
.18 \\
.01\end{array}$ \\
\hline $\begin{array}{l}21 \\
22 \\
23 \\
24 \\
25\end{array}$ & $\begin{array}{r}.12 \\
2.49 \\
.04 \\
.00 \\
.00\end{array}$ & $\begin{array}{l}.02 \\
.01 \\
.00 \\
.11 \\
.00\end{array}$ & $\begin{array}{l}.38 \\
.02 \\
.00 \\
.00 \\
.00\end{array}$ & $\begin{array}{l}.40 \\
.02 \\
.00 \\
.00 \\
.06\end{array}$ & $\begin{array}{r}.51 \\
1.53 \\
.00 \\
.00 \\
.00\end{array}$ & $\begin{array}{l}.00 \\
.00 \\
.00 \\
.01 \\
.00\end{array}$ & $\begin{array}{l}.00 \\
.03 \\
.11 \\
.00 \\
.00\end{array}$ & $\begin{array}{r}.00 \\
.01 \\
4.81 \\
.16 \\
.00\end{array}$ & $\begin{array}{l}.04 \\
.03 \\
.84 \\
.00 \\
.45\end{array}$ & $\begin{array}{l}.74 \\
.59 \\
.46 \\
.14 \\
.00\end{array}$ & $\begin{array}{r}.88 \\
.02 \\
.00 \\
1.37 \\
1.28\end{array}$ & $\begin{array}{l}.00 \\
.00 \\
.13 \\
.00 \\
.00\end{array}$ \\
\hline $\begin{array}{l}26 \\
27 \\
28 \\
29 \\
30 \\
31\end{array}$ & $\begin{array}{l}.00 \\
.43 \\
.00 \\
.00 \\
.00 \\
.09\end{array}$ & $\begin{array}{l}.00 \\
.00 \\
.00 \\
.67 \\
.00 \\
-.-\end{array}$ & $\begin{array}{r}.00 \\
.00 \\
.17 \\
2.49 \\
.00 \\
.00\end{array}$ & $\begin{array}{l}.05 \\
.00 \\
.00 \\
.00 \\
.00 \\
.00\end{array}$ & $\begin{array}{l}.00 \\
.63 \\
.00 \\
.00 \\
-.- \\
.--\end{array}$ & $\begin{array}{l}.12 \\
.45 \\
.00 \\
.00 \\
.00 \\
.00\end{array}$ & $\begin{array}{l}.00 \\
.00 \\
.00 \\
.00 \\
.00 \\
.--\end{array}$ & $\begin{array}{l}.77 \\
.50 \\
.00 \\
.00 \\
.06 \\
.00\end{array}$ & $\begin{array}{l}.00 \\
.02 \\
.33 \\
.52 \\
.82 \\
.--\end{array}$ & $\begin{array}{l}.00 \\
.00 \\
.00 \\
.00 \\
.19 \\
.11\end{array}$ & $\begin{array}{l}.00 \\
.01 \\
.00 \\
.00 \\
.00 \\
.00\end{array}$ & $\begin{array}{r}.00 \\
4.06 \\
.01 \\
.00 \\
.00 \\
-\end{array}$ \\
\hline
\end{tabular}

Site 14--Florida Power and Light

[Water year October 1984 to September 1985]

\begin{tabular}{|c|c|c|c|c|c|c|c|c|c|c|c|c|}
\hline $\begin{array}{l}1 \\
2 \\
3 \\
4 \\
5\end{array}$ & $\begin{array}{r}0.09 \\
.00 \\
.00 \\
.01 \\
.00\end{array}$ & $\begin{array}{r}0.00 \\
.01 \\
.02 \\
.00 \\
.00\end{array}$ & $\begin{array}{r}0.00 \\
.00 \\
.00 \\
.00 \\
.39\end{array}$ & $\begin{array}{r}0.00 \\
.02 \\
1.39 \\
.01 \\
.00\end{array}$ & $\begin{array}{r}0.00 \\
.02 \\
.01 \\
.00 \\
.00\end{array}$ & $\begin{array}{r}0.00 \\
.00 \\
.00 \\
.01 \\
.00\end{array}$ & $\begin{array}{r}0.00 \\
.00 \\
.00 \\
.00 \\
.02\end{array}$ & $\begin{array}{r}0.00 \\
.00 \\
.00 \\
3.53 \\
.00\end{array}$ & $\begin{array}{r}0.00 \\
.00 \\
.00 \\
.00 \\
.00\end{array}$ & $\begin{array}{r}0.00 \\
.00 \\
.00 \\
.00 \\
.95\end{array}$ & $\begin{array}{l}--- \\
--- \\
--- \\
---\end{array}$ & $\begin{array}{l}--- \\
--- \\
--- \\
---\end{array}$ \\
\hline $\begin{array}{r}6 \\
7 \\
8 \\
9 \\
10\end{array}$ & $\begin{array}{l}.00 \\
.00 \\
.12 \\
.00 \\
.13\end{array}$ & $\begin{array}{l}.00 \\
.01 \\
.00 \\
.00 \\
.00\end{array}$ & $\begin{array}{l}.00 \\
.00 \\
.01 \\
.00 \\
.01\end{array}$ & $\begin{array}{l}.00 \\
.01 \\
.00 \\
.00 \\
.01\end{array}$ & $\begin{array}{l}.44 \\
.16 \\
.08 \\
.00 \\
.01\end{array}$ & $\begin{array}{l}.00 \\
.00 \\
.00 \\
.00 \\
.00\end{array}$ & $\begin{array}{l}.69 \\
.01 \\
.00 \\
.00 \\
.00\end{array}$ & $\begin{array}{l}.00 \\
.00 \\
.00 \\
.00 \\
.00\end{array}$ & $\begin{array}{l}.00 \\
.02 \\
.20 \\
.00 \\
.08\end{array}$ & $\begin{array}{r}.01 \\
1.33 \\
.00 \\
.00 \\
.00\end{array}$ & $\begin{array}{l}--- \\
--- \\
--- \\
---\end{array}$ & $\begin{array}{l}--- \\
--- \\
--- \\
---\end{array}$ \\
\hline $\begin{array}{l}11 \\
12 \\
13 \\
14 \\
15\end{array}$ & $\begin{array}{l}.11 \\
.00 \\
.00 \\
.00 \\
.00\end{array}$ & $\begin{array}{l}.00 \\
.00 \\
.00 \\
.00 \\
.01\end{array}$ & $\begin{array}{l}.00 \\
.02 \\
.00 \\
.00 \\
.00\end{array}$ & $\begin{array}{l}.00 \\
.00 \\
.00 \\
.01 \\
.01\end{array}$ & $\begin{array}{l}.15 \\
.04 \\
.00 \\
.00 \\
.02\end{array}$ & $\begin{array}{l}.00 \\
.00 \\
.00 \\
.00 \\
.00\end{array}$ & $\begin{array}{r}.00 \\
1.28 \\
.23 \\
.09 \\
.00\end{array}$ & $\begin{array}{l}.00 \\
.00 \\
.00 \\
.00 \\
.00\end{array}$ & $\begin{array}{r}.00 \\
.75 \\
1.43 \\
.63 \\
1.93\end{array}$ & $\begin{array}{l}.17 \\
.76 \\
.00 \\
.00 \\
.01\end{array}$ & $\begin{array}{l}--- \\
--- \\
--- \\
--- \\
---\end{array}$ & $\begin{array}{l}--- \\
--- \\
--- \\
---\end{array}$ \\
\hline $\begin{array}{l}16 \\
17 \\
18 \\
19 \\
20\end{array}$ & $\begin{array}{l}.00 \\
.00 \\
.00 \\
.00 \\
.00\end{array}$ & $\begin{array}{l}.00 \\
.00 \\
.00 \\
.00 \\
.00\end{array}$ & $\begin{array}{l}.00 \\
.00 \\
.02 \\
.00 \\
.01\end{array}$ & $\begin{array}{l}.00 \\
.05 \\
.17 \\
.00 \\
.02\end{array}$ & $\begin{array}{l}.00 \\
.00 \\
.00 \\
.00 \\
.00\end{array}$ & $\begin{array}{r}.00 \\
.00 \\
- \\
- \\
1.25\end{array}$ & $\begin{array}{l}.00 \\
.00 \\
-- \\
--- \\
--\end{array}$ & $\begin{array}{r}.00 \\
.00 \\
.00 \\
.00 \\
2.09\end{array}$ & $\begin{array}{l}.26 \\
.00 \\
.00 \\
.00 \\
.01\end{array}$ & $\begin{array}{r}1.02 \\
.00 \\
- \\
-- \\
--\end{array}$ & $\begin{array}{l}--- \\
--- \\
--- \\
---\end{array}$ & $\begin{array}{l}--- \\
--- \\
--- \\
---\end{array}$ \\
\hline $\begin{array}{l}21 \\
22 \\
23 \\
24 \\
25\end{array}$ & $\begin{array}{l}.32 \\
.01 \\
.20 \\
.00 \\
.00\end{array}$ & $\begin{array}{r}.13 \\
2.27 \\
2.54 \\
.01 \\
.02\end{array}$ & $\begin{array}{l}.00 \\
.00 \\
.00 \\
.02 \\
.00\end{array}$ & $\begin{array}{l}.00 \\
.00 \\
.00 \\
.00 \\
.00\end{array}$ & $\begin{array}{l}.00 \\
.00 \\
.00 \\
.00 \\
.00\end{array}$ & $\begin{array}{l}.01 \\
.00 \\
.00 \\
.00 \\
.00\end{array}$ & $\begin{array}{l}--- \\
--- \\
--- \\
--- \\
---\end{array}$ & $\begin{array}{r}.01 \\
.59 \\
1.66 \\
.01 \\
.00\end{array}$ & $\begin{array}{l}.02 \\
.01 \\
.00 \\
.01 \\
.00\end{array}$ & $\begin{array}{l}--- \\
--- \\
--- \\
---\end{array}$ & $\begin{array}{l}--- \\
--- \\
--- \\
---\end{array}$ & $\overline{-}$ \\
\hline $\begin{array}{l}26 \\
27 \\
28 \\
29 \\
30 \\
31\end{array}$ & $\begin{array}{l}.24 \\
.01 \\
.00 \\
.00 \\
.13 \\
.00\end{array}$ & $\begin{array}{l}.01 \\
.02 \\
.01 \\
.00 \\
.01 \\
.0-\end{array}$ & $\begin{array}{l}.00 \\
.07 \\
.00 \\
.02 \\
.00 \\
.00\end{array}$ & $\begin{array}{l}.00 \\
.00 \\
.00 \\
.00 \\
.00 \\
.00\end{array}$ & $\begin{array}{l}.00 \\
.00 \\
.00 \\
--- \\
--- \\
---\end{array}$ & $\begin{array}{l}.00 \\
.00 \\
.00 \\
.00 \\
.00 \\
.14\end{array}$ & $\begin{array}{l}--- \\
--- \\
--- \\
-0 \\
-00 \\
---\end{array}$ & $\begin{array}{l}.00 \\
.00 \\
.00 \\
.00 \\
.00 \\
.00\end{array}$ & $\begin{array}{l}.01 \\
.01 \\
.41 \\
.13 \\
.26 \\
.--\end{array}$ & $\begin{array}{l}--- \\
--- \\
--- \\
--- \\
---\end{array}$ & $\begin{array}{l}--- \\
--- \\
--- \\
---\end{array}$ & $\begin{array}{l}-- \\
--- \\
-- \\
-- \\
--\end{array}$ \\
\hline
\end{tabular}


APPENDIX I--Daily rainfall--Continued [Rainfall in inches]

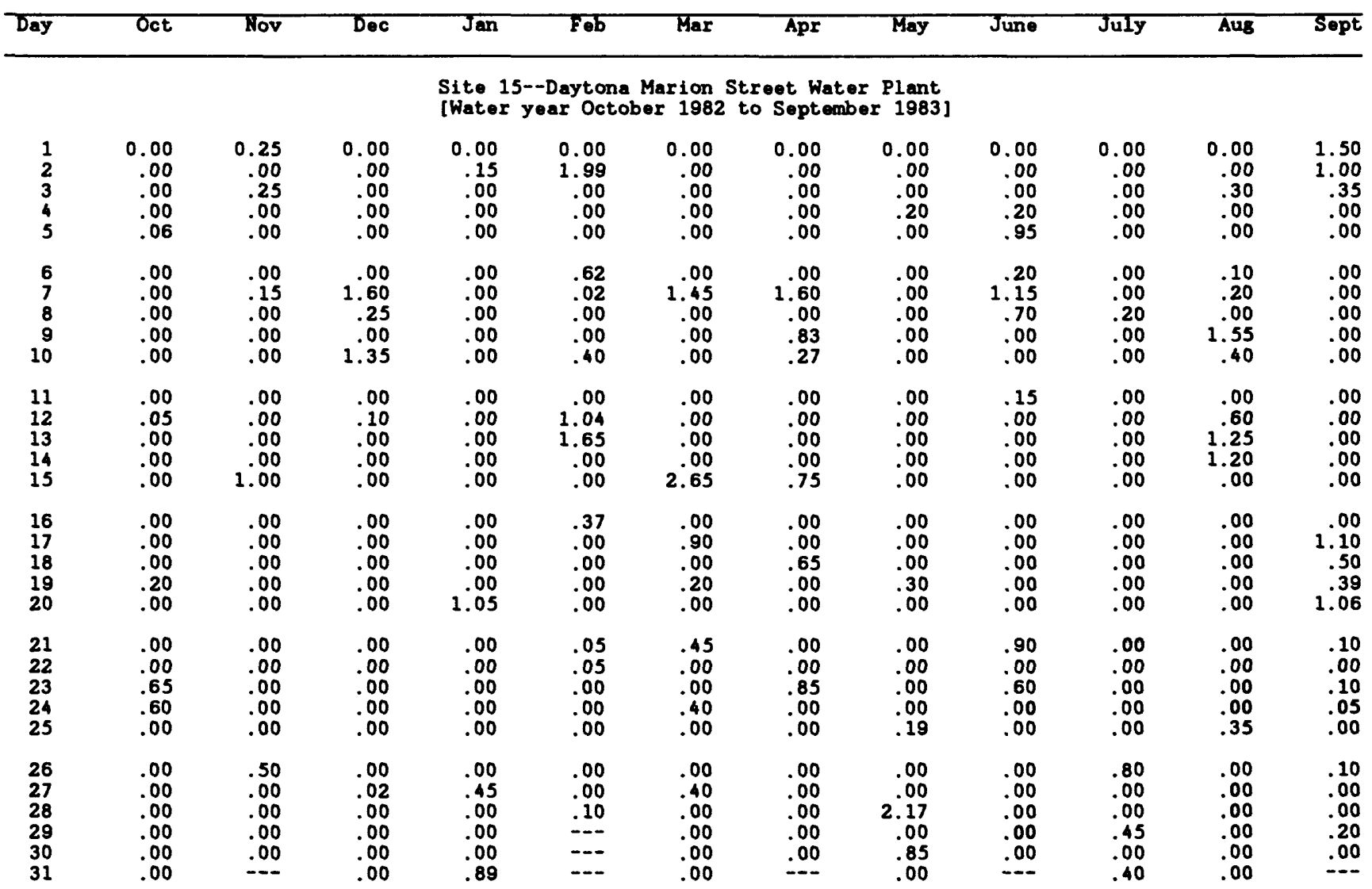

Site 15--Daytona Marion Street Water Plant [Water year October 1983 to September 1984]

\begin{tabular}{|c|c|c|c|c|c|c|c|c|c|c|c|c|}
\hline $\begin{array}{l}1 \\
2 \\
3 \\
4 \\
5\end{array}$ & $\begin{array}{r}0.00 \\
.00 \\
.00 \\
.00 \\
.00\end{array}$ & $\begin{array}{l}-\cdots \\
-- \\
-\cdots \\
-\cdots\end{array}$ & $\begin{array}{r}0.00 \\
.00 \\
.00 \\
.02 \\
.00\end{array}$ & $\begin{array}{r}0.00 \\
.00 \\
.00 \\
.00 \\
.00\end{array}$ & $\begin{array}{r}0.00 \\
.05 \\
.05 \\
.00 \\
.00\end{array}$ & $\begin{array}{r}0.00 \\
.00 \\
.00 \\
.00 \\
.00\end{array}$ & $\begin{array}{r}0.00 \\
.00 \\
2.40 \\
.90 \\
.00\end{array}$ & $\begin{array}{r}0.00 \\
.00 \\
.00 \\
.00 \\
.00\end{array}$ & $\begin{array}{r}0.00 \\
.00 \\
.00 \\
.00 \\
.00\end{array}$ & $\begin{array}{r}0.80 \\
.65 \\
.65 \\
.00 \\
.00\end{array}$ & $\begin{array}{r}0.00 \\
.00 \\
.00 \\
.00 \\
.00\end{array}$ & $\begin{array}{r}0.00 \\
.00 \\
.00 \\
.00 \\
1.20\end{array}$ \\
\hline $\begin{array}{r}6 \\
7 \\
8 \\
9 \\
10\end{array}$ & $\begin{array}{l}.00 \\
.00 \\
.00 \\
.50 \\
.10\end{array}$ & $\begin{array}{l}.15 \\
.00 \\
.00\end{array}$ & $\begin{array}{l}.00 \\
.00 \\
.00 \\
.00 \\
.00\end{array}$ & $\begin{array}{l}.00 \\
.00 \\
.00 \\
.00 \\
.20\end{array}$ & $\begin{array}{l}.00 \\
.00 \\
.00 \\
.00 \\
.00\end{array}$ & $\begin{array}{l}.00 \\
.00 \\
.00 \\
.00 \\
.00\end{array}$ & $\begin{array}{l}.00 \\
.00 \\
.00 \\
.10 \\
.00\end{array}$ & $\begin{array}{r}.00 \\
1.25 \\
.00 \\
.00 \\
.00\end{array}$ & $\begin{array}{l}.00 \\
.00 \\
.00 \\
.00 \\
.00\end{array}$ & $\begin{array}{l}.00 \\
.00 \\
.00 \\
.00 \\
.00\end{array}$ & $\begin{array}{l}.00 \\
.00 \\
.00 \\
.00 \\
.00\end{array}$ & $\begin{array}{r}.00 \\
.30 \\
.00 \\
.30 \\
.97\end{array}$ \\
\hline $\begin{array}{l}11 \\
12 \\
13 \\
14 \\
15\end{array}$ & $\begin{array}{r}1.70 \\
.00 \\
.13 \\
.00 \\
1.55\end{array}$ & $\begin{array}{l}.05 \\
.00 \\
.00 \\
.00 \\
.19\end{array}$ & $\begin{array}{r}2.46 \\
2.78 \\
.00 \\
.00 \\
.12\end{array}$ & $\begin{array}{l}.00 \\
.00 \\
.00 \\
.00 \\
.00\end{array}$ & $\begin{array}{l}.00 \\
.25 \\
.65 \\
.00 \\
.00\end{array}$ & $\begin{array}{l}.00 \\
.00 \\
.05 \\
.00 \\
.00\end{array}$ & $\begin{array}{l}.00 \\
.00 \\
.00 \\
.95 \\
.00\end{array}$ & $\begin{array}{l}.00 \\
.00 \\
.00 \\
.00 \\
.00\end{array}$ & $\begin{array}{l}.00 \\
.45 \\
.00 \\
.00 \\
.00\end{array}$ & $\begin{array}{l}.00 \\
.00 \\
.00 \\
.00 \\
.00\end{array}$ & $\begin{array}{l}.05 \\
.45 \\
.00 \\
.00 \\
.00\end{array}$ & $\begin{array}{l}.00 \\
.00 \\
.00 \\
.00 \\
.00\end{array}$ \\
\hline $\begin{array}{l}16 \\
17 \\
18 \\
19 \\
20\end{array}$ & $\begin{array}{r}.60 \\
1.15 \\
.00 \\
.00 \\
.00\end{array}$ & $\begin{array}{l}.03 \\
.00 \\
.00 \\
.00 \\
.55\end{array}$ & $\begin{array}{r}.00 \\
.30 \\
1.04 \\
.00 \\
4.45\end{array}$ & $\begin{array}{l}.00 \\
.00 \\
.02 \\
.35 \\
.40\end{array}$ & $\begin{array}{l}.00 \\
.00 \\
.00 \\
.00 \\
.00\end{array}$ & $\begin{array}{l}.00 \\
.00 \\
.12 \\
.00 \\
.60\end{array}$ & $\begin{array}{l}.00 \\
.00 \\
.00 \\
.00 \\
.00\end{array}$ & $\begin{array}{l}.00 \\
.00 \\
.00 \\
.00 \\
.00\end{array}$ & $\begin{array}{l}.00 \\
.05 \\
.00 \\
.00 \\
.00\end{array}$ & $\begin{array}{l}.00 \\
.00 \\
.00 \\
.11 \\
.70\end{array}$ & $\begin{array}{l}.00 \\
.32 \\
.45 \\
.00 \\
.15\end{array}$ & $\begin{array}{l}.00 \\
.15 \\
.90 \\
.00 \\
.10\end{array}$ \\
\hline $\begin{array}{l}21 \\
22 \\
23 \\
24 \\
25\end{array}$ & $\begin{array}{l}.00 \\
.98 \\
.10 \\
.00 \\
.00\end{array}$ & $\begin{array}{l}.02 \\
.00 \\
.00 \\
.15 \\
.00\end{array}$ & $\begin{array}{l}.95 \\
.00 \\
.00 \\
.00 \\
.00\end{array}$ & $\begin{array}{l}.45 \\
.00 \\
.00 \\
.00 \\
.00\end{array}$ & $\begin{array}{r}.35 \\
1.75 \\
.00 \\
.00 \\
.00\end{array}$ & $\begin{array}{l}.00 \\
.00 \\
.00 \\
.02 \\
.00\end{array}$ & $\begin{array}{l}.00 \\
.00 \\
.05 \\
.00 \\
.00\end{array}$ & $\begin{array}{r}.00 \\
.00 \\
3.15 \\
.00 \\
.00\end{array}$ & $\begin{array}{l}.00 \\
.48 \\
.58 \\
.00 \\
.28\end{array}$ & $\begin{array}{l}.80 \\
.45 \\
.10 \\
.00 \\
.00\end{array}$ & $\begin{array}{l}.38 \\
.00 \\
.00 \\
.00 \\
.73\end{array}$ & $\begin{array}{l}.00 \\
.00 \\
.20 \\
.00 \\
.00\end{array}$ \\
\hline $\begin{array}{l}26 \\
27 \\
28 \\
29 \\
30\end{array}$ & $\begin{array}{l}.00 \\
.00 \\
.00 \\
.00 \\
.00 \\
.00\end{array}$ & $\begin{array}{l}.00 \\
.00 \\
.00 \\
.55 \\
.00 \\
.--\end{array}$ & $\begin{array}{r}.00 \\
.00 \\
.15 \\
2.00 \\
.00 \\
.00\end{array}$ & $\begin{array}{l}.00 \\
.05 \\
.00 \\
.00 \\
.00 \\
.00\end{array}$ & $\begin{array}{l}.00 \\
.35 \\
.00 \\
.00 \\
. .-\end{array}$ & $\begin{array}{l}.00 \\
.35 \\
.00 \\
.00 \\
.00 \\
.00\end{array}$ & $\begin{array}{l}.00 \\
.00 \\
.00 \\
.00 \\
.00 \\
.--\end{array}$ & $\begin{array}{l}.65 \\
.00 \\
.00 \\
.00 \\
.02 \\
.00\end{array}$ & $\begin{array}{l}.00 \\
.00 \\
.15 \\
.55 \\
.75 \\
.\end{array}$ & $\begin{array}{l}.00 \\
.00 \\
.00 \\
.00 \\
.10 \\
.00\end{array}$ & $\begin{array}{l}.00 \\
.00 \\
.00 \\
.00 \\
.00 \\
.00\end{array}$ & $\begin{array}{r}.00 \\
1.01 \\
1.60 \\
.00 \\
.00 \\
-0\end{array}$ \\
\hline
\end{tabular}




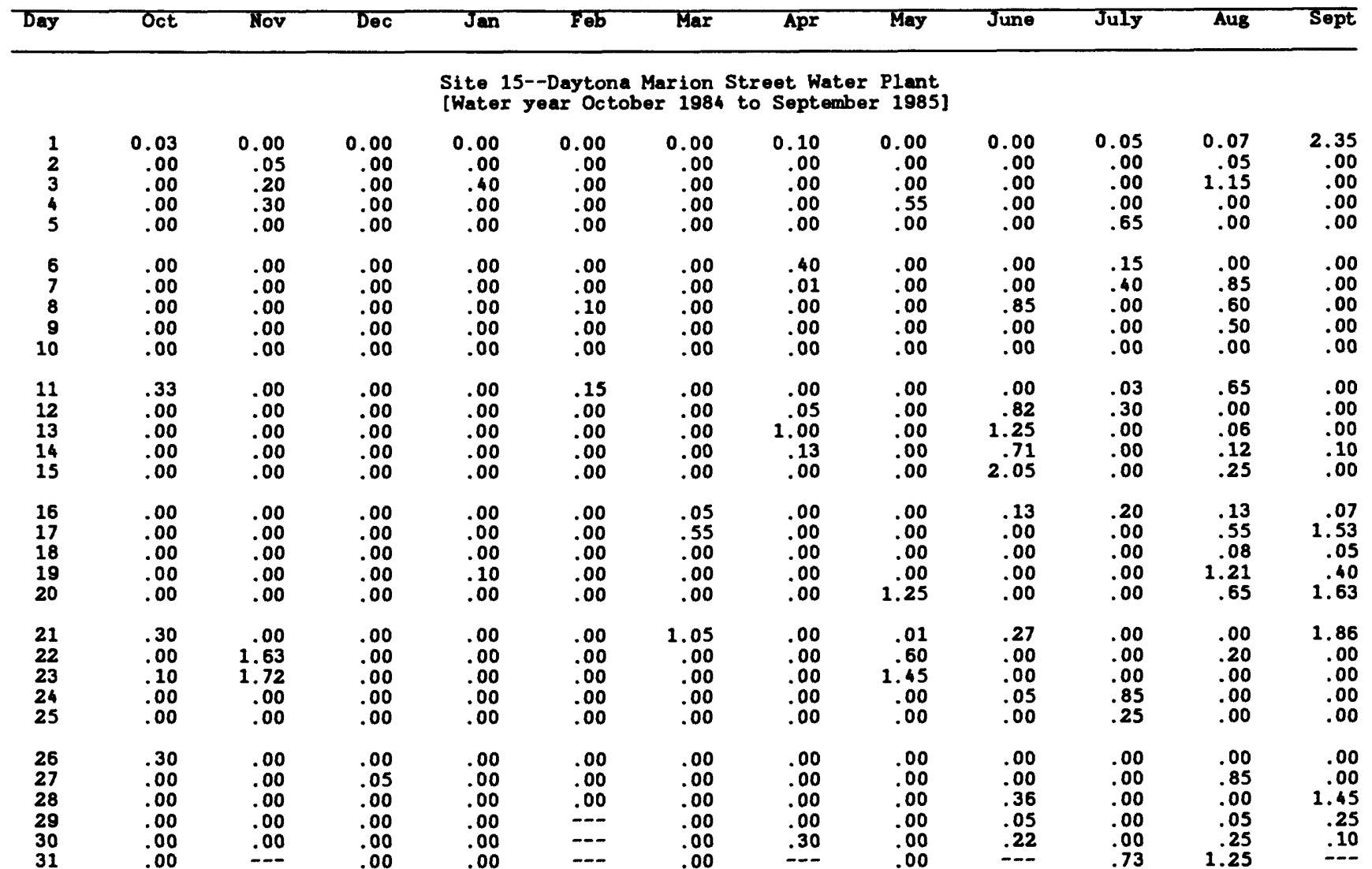

Site 16--Daytona Brennan Water Plant

[Water year October 1982 to September 1983]

\begin{tabular}{|c|c|c|c|c|c|c|c|c|c|c|c|c|}
\hline $\begin{array}{l}1 \\
2 \\
3 \\
4 \\
5\end{array}$ & $\begin{array}{r}0.00 \\
.00 \\
.00 \\
.00 \\
.11\end{array}$ & $\begin{array}{r}0.02 \\
.20 \\
.23 \\
.08 \\
.00\end{array}$ & $\begin{array}{r}0.00 \\
.00 \\
.00 \\
.00 \\
.05\end{array}$ & $\begin{array}{r}0.00 \\
.25 \\
.00 \\
.00 \\
.00\end{array}$ & $\begin{array}{r}0.00 \\
2.75 \\
.00 \\
.00 \\
.00\end{array}$ & $\begin{array}{r}0.00 \\
.00 \\
.00 \\
.00 \\
.00\end{array}$ & $\begin{array}{r}0.12 \\
.00 \\
.00 \\
.00 \\
.00\end{array}$ & $\begin{array}{r}0.00 \\
.00 \\
.00 \\
.26 \\
.00\end{array}$ & $\begin{array}{r}0.00 \\
.00 \\
.00 \\
.80 \\
.24\end{array}$ & $\begin{array}{r}0.00 \\
.05 \\
.00 \\
.00 \\
.00\end{array}$ & $\begin{array}{r}0.00 \\
.00 \\
.00 \\
.25 \\
.00\end{array}$ & $\begin{array}{r}1.00 \\
.52 \\
.71 \\
.00 \\
.00\end{array}$ \\
\hline $\begin{array}{r}6 \\
7 \\
8 \\
9 \\
10\end{array}$ & $\begin{array}{l}.01 \\
.00 \\
.00 \\
.00 \\
.10\end{array}$ & $\begin{array}{l}.00 \\
.07 \\
.04 \\
.05 \\
.01\end{array}$ & $\begin{array}{l}.00 \\
.04 \\
.20 \\
.15 \\
.40\end{array}$ & $\begin{array}{l}.00 \\
.00 \\
.00 \\
.00 \\
.00\end{array}$ & $\begin{array}{l}.70 \\
.03 \\
.00 \\
.00 \\
.39\end{array}$ & $\begin{array}{r}.00 \\
1.87 \\
.01 \\
.00 \\
.00\end{array}$ & $\begin{array}{l}.00 \\
.66 \\
.02 \\
.99 \\
.16\end{array}$ & $\begin{array}{l}.00 \\
.00 \\
.00 \\
.00 \\
.00\end{array}$ & $\begin{array}{r}.54 \\
1.55 \\
.22 \\
.00 \\
.00\end{array}$ & $\begin{array}{l}.00 \\
.00 \\
.04 \\
.00 \\
.00\end{array}$ & $\begin{array}{r}.25 \\
.25 \\
.04 \\
1.20 \\
.12\end{array}$ & $\begin{array}{l}.00 \\
.00 \\
.00 \\
.00 \\
.00\end{array}$ \\
\hline $\begin{array}{l}11 \\
12 \\
13 \\
14 \\
15\end{array}$ & $\begin{array}{l}.40 \\
.02 \\
.00 \\
.40 \\
.01\end{array}$ & $\begin{array}{l}.00 \\
.00 \\
.00 \\
.00 \\
.08\end{array}$ & $\begin{array}{l}.00 \\
.16 \\
.00 \\
.00 \\
.01\end{array}$ & $\begin{array}{l}.00 \\
.00 \\
.00 \\
.00 \\
.00\end{array}$ & $\begin{array}{r}.02 \\
.80 \\
1.50 \\
.01 \\
.00\end{array}$ & $\begin{array}{r}.00 \\
.00 \\
.00 \\
.00 \\
1.80\end{array}$ & $\begin{array}{r}.00 \\
.00 \\
.00 \\
.00 \\
1.80\end{array}$ & $\begin{array}{l}.60 \\
.00 \\
.00 \\
.00 \\
.00\end{array}$ & $\begin{array}{l}.00 \\
.00 \\
.42 \\
.07 \\
.00\end{array}$ & $\begin{array}{l}.00 \\
.00 \\
.00 \\
.00 \\
.00\end{array}$ & $\begin{array}{l}.00 \\
.10 \\
.62 \\
.25 \\
.00\end{array}$ & $\begin{array}{l}.00 \\
.00 \\
.00 \\
.13 \\
.00\end{array}$ \\
\hline $\begin{array}{l}16 \\
17 \\
18 \\
19 \\
20\end{array}$ & $\begin{array}{l}.00 \\
.00 \\
.00 \\
.02 \\
.00\end{array}$ & $\begin{array}{l}.04 \\
.05 \\
.02 \\
.04 \\
.00\end{array}$ & $\begin{array}{l}.15 \\
.00 \\
.00 \\
.00 \\
.00\end{array}$ & $\begin{array}{r}.00 \\
.00 \\
.00 \\
.00 \\
1.60\end{array}$ & $\begin{array}{l}.50 \\
.01 \\
.00 \\
.00 \\
.00\end{array}$ & $\begin{array}{r}.01 \\
1.27 \\
.02 \\
.00 \\
.00\end{array}$ & $\begin{array}{l}.00 \\
.00 \\
.66 \\
.00 \\
.00\end{array}$ & $\begin{array}{l}.00 \\
.00 \\
.00 \\
.16 \\
.00\end{array}$ & $\begin{array}{l}.00 \\
.00 \\
.00 \\
.00 \\
.00\end{array}$ & $\begin{array}{l}.00 \\
.00 \\
.00 \\
.00 \\
.00\end{array}$ & $\begin{array}{l}.00 \\
.04 \\
.00 \\
.00 \\
.00\end{array}$ & $\begin{array}{l}.44 \\
.42 \\
.00 \\
.62 \\
.15\end{array}$ \\
\hline $\begin{array}{l}21 \\
22 \\
23 \\
24 \\
25\end{array}$ & $\begin{array}{r}.00 \\
.00 \\
2.00 \\
.03 \\
.00\end{array}$ & $\begin{array}{l}.00 \\
.00 \\
.00 \\
.00 \\
.00\end{array}$ & $\begin{array}{l}.00 \\
.00 \\
.00 \\
.00 \\
.00\end{array}$ & $\begin{array}{l}.09 \\
.15 \\
.15 \\
.00 \\
.00\end{array}$ & $\begin{array}{l}.00 \\
.01 \\
.00 \\
.00 \\
.00\end{array}$ & $\begin{array}{r}1.10 \\
.00 \\
.00 \\
.44 \\
.00\end{array}$ & $\begin{array}{l}.00 \\
.00 \\
.94 \\
.00 \\
.00\end{array}$ & $\begin{array}{l}.00 \\
.00 \\
.00 \\
.00 \\
.07\end{array}$ & $\begin{array}{r}1.05 \\
.65 \\
1.40 \\
.03 \\
.00\end{array}$ & $\begin{array}{l}.00 \\
.00 \\
.00 \\
.00 \\
.00\end{array}$ & $\begin{array}{l}.00 \\
.00 \\
.00 \\
.23 \\
.00\end{array}$ & $\begin{array}{r}.18 \\
.00 \\
.00 \\
1.40 \\
.04\end{array}$ \\
\hline $\begin{array}{l}26 \\
27 \\
28 \\
29 \\
30 \\
31\end{array}$ & $\begin{array}{l}.00 \\
.00 \\
.00 \\
.00 \\
.00 \\
.00\end{array}$ & $\begin{array}{l}.36 \\
.02 \\
.00 \\
.00 \\
.00 \\
-.-0\end{array}$ & $\begin{array}{l}.00 \\
.04 \\
.00 \\
.00 \\
.00 \\
.00\end{array}$ & $\begin{array}{r}.00 \\
.48 \\
.00 \\
.00 \\
.00 \\
1.10\end{array}$ & $\begin{array}{l}.00 \\
.02 \\
.05 \\
-.- \\
-.-\end{array}$ & $\begin{array}{l}.00 \\
.51 \\
.00 \\
.00 \\
.00 \\
.00\end{array}$ & $\begin{array}{l}.00 \\
.00 \\
.00 \\
.00 \\
.00 \\
-.-\end{array}$ & $\begin{array}{r}.00 \\
.00 \\
.00 \\
.84 \\
1.32 \\
.00\end{array}$ & $\begin{array}{l}.00 \\
.00 \\
.00 \\
.70 \\
.20 \\
-.--\end{array}$ & $\begin{array}{r}.36 \\
.65 \\
.00 \\
.45 \\
.00 \\
1.06\end{array}$ & $\begin{array}{l}.00 \\
.00 \\
.05 \\
.00 \\
.00 \\
.00\end{array}$ & $\begin{array}{l}.10 \\
.00 \\
.00 \\
.00 \\
.00 \\
.0\end{array}$ \\
\hline
\end{tabular}


APPENDIX I--Daily rainfal1--Continued

[Rainfall in inches]

\begin{tabular}{|c|c|c|c|c|c|c|c|c|c|c|c|c|}
\hline Day & oct & Nov & Dec & Jan & Fob & Mar & Apr & May & June & July & Aug & Sept \\
\hline \multicolumn{13}{|c|}{$\begin{array}{l}\text { Site 16--Daytona Brennan Water Plant } \\
\text { [Water year October } 1983 \text { to September 1984] }\end{array}$} \\
\hline $\begin{array}{l}1 \\
2 \\
3 \\
4 \\
5\end{array}$ & $\begin{array}{l}0.00 \\
.00 \\
.00 \\
.00 \\
.00\end{array}$ & $\begin{array}{l}0.18 \\
.00 \\
.00 \\
.00 \\
.20\end{array}$ & $\begin{array}{l}0.00 \\
.00 \\
.00 \\
.05 \\
.00\end{array}$ & $\begin{array}{l}0.00 \\
.00 \\
.00 \\
.00 \\
.00\end{array}$ & $\begin{array}{r}0.00 \\
.06 \\
.00 \\
.00 \\
.00\end{array}$ & $\begin{array}{l}0.00 \\
.00 \\
.00 \\
.00 \\
.00\end{array}$ & $\begin{array}{r}0.00 \\
.00 \\
3.20 \\
1.86 \\
.00\end{array}$ & $\begin{array}{r}0.00 \\
.00 \\
.00 \\
.00 \\
.02\end{array}$ & $\begin{array}{l}0.00 \\
.00 \\
.00 \\
.00 \\
.00\end{array}$ & $\begin{array}{r}1.65 \\
.80 \\
1.00 \\
.00 \\
.00\end{array}$ & $\begin{array}{l}0.07 \\
.00 \\
.00 \\
.00 \\
.00\end{array}$ & $\begin{array}{r}0.00 \\
.00 \\
.00 \\
.70 \\
2.90\end{array}$ \\
\hline $\begin{array}{r}6 \\
7 \\
8 \\
9 \\
10\end{array}$ & $\begin{array}{r}.00 \\
.00 \\
1.30 \\
.28 \\
.07\end{array}$ & $\begin{array}{l}.01 \\
.04 \\
.00 \\
.30 \\
.00\end{array}$ & $\begin{array}{l}.00 \\
.00 \\
.00 \\
.00 \\
.00\end{array}$ & $\begin{array}{l}.00 \\
.00 \\
.00 \\
.00 \\
.20\end{array}$ & $\begin{array}{l}.00 \\
.00 \\
.00 \\
.00 \\
.00\end{array}$ & $\begin{array}{l}.00 \\
.02 \\
.00 \\
.00 \\
.00\end{array}$ & $\begin{array}{l}.00 \\
.00 \\
.00 \\
.25 \\
.00\end{array}$ & $\begin{array}{l}.00 \\
.00 \\
.00 \\
.03 \\
.00\end{array}$ & $\begin{array}{l}.00 \\
.00 \\
.00 \\
.00 \\
.00\end{array}$ & $\begin{array}{l}.00 \\
.08 \\
.00 \\
.00 \\
.00\end{array}$ & $\begin{array}{l}.00 \\
.00 \\
.00 \\
.00 \\
.00\end{array}$ & $\begin{array}{r}1.00 \\
1.40 \\
.10 \\
2.30 \\
.80\end{array}$ \\
\hline $\begin{array}{l}11 \\
12 \\
13 \\
14 \\
15\end{array}$ & $\begin{array}{r}.72 \\
.02 \\
.10 \\
.00 \\
2.47\end{array}$ & $\begin{array}{l}.08 \\
.00 \\
.00 \\
.00 \\
.34\end{array}$ & $\begin{array}{r}2.77 \\
1.70 \\
.00 \\
.05 \\
.17\end{array}$ & $\begin{array}{l}.00 \\
.00 \\
.00 \\
.01 \\
.00\end{array}$ & $\begin{array}{r}.00 \\
.70 \\
1.28 \\
.00 \\
.00\end{array}$ & $\begin{array}{l}.00 \\
.14 \\
.00 \\
.00 \\
.00\end{array}$ & $\begin{array}{l}.00 \\
.00 \\
.00 \\
.74 \\
.00\end{array}$ & $\begin{array}{l}.00 \\
.00 \\
.00 \\
.00 \\
.00\end{array}$ & $\begin{array}{l}.00 \\
.00 \\
.60 \\
.00 \\
.00\end{array}$ & $\begin{array}{l}.00 \\
.00 \\
.70 \\
.00 \\
.19\end{array}$ & $\begin{array}{l}.00 \\
.00 \\
.00 \\
.00 \\
.00\end{array}$ & $\begin{array}{l}.00 \\
.00 \\
.00 \\
.00 \\
.00\end{array}$ \\
\hline $\begin{array}{l}16 \\
17 \\
18 \\
19 \\
20\end{array}$ & $\begin{array}{r}.34 \\
2.50 \\
.02 \\
.00 \\
.00\end{array}$ & $\begin{array}{l}.00 \\
.00 \\
.00 \\
.00 \\
.77\end{array}$ & $\begin{array}{r}.00 \\
.01 \\
.90 \\
.09 \\
1.92\end{array}$ & $\begin{array}{l}.00 \\
.00 \\
.02 \\
.47 \\
.45\end{array}$ & $\begin{array}{l}.00 \\
.00 \\
.00 \\
.00 \\
.00\end{array}$ & $\begin{array}{l}.00 \\
.00 \\
.05 \\
.00 \\
.57\end{array}$ & $\begin{array}{l}.00 \\
.00 \\
.00 \\
.00 \\
.00\end{array}$ & $\begin{array}{l}.00 \\
.00 \\
.00 \\
.00 \\
.00\end{array}$ & $\begin{array}{l}.00 \\
.00 \\
.00 \\
.00 \\
.00\end{array}$ & $\begin{array}{l}.00 \\
.05 \\
.00 \\
.92 \\
.90\end{array}$ & $\begin{array}{l}.00 \\
.80 \\
.15 \\
.00 \\
.30\end{array}$ & $\begin{array}{r}.00 \\
.22 \\
1.05 \\
.07 \\
.00\end{array}$ \\
\hline $\begin{array}{l}21 \\
22 \\
23 \\
24 \\
25\end{array}$ & $\begin{array}{r}.02 \\
1.56 \\
.05 \\
.00 \\
.00\end{array}$ & $\begin{array}{l}.00 \\
.00 \\
.00 \\
.18 \\
.00\end{array}$ & $\begin{array}{l}.37 \\
.00 \\
.00 \\
.00 \\
.00\end{array}$ & $\begin{array}{l}.17 \\
.00 \\
.00 \\
.00 \\
.04\end{array}$ & $\begin{array}{r}.29 \\
1.60 \\
.00 \\
.00 \\
.00\end{array}$ & $\begin{array}{l}.00 \\
.00 \\
.00 \\
.00 \\
.00\end{array}$ & $\begin{array}{l}.00 \\
.00 \\
.26 \\
.00 \\
.00\end{array}$ & $\begin{array}{r}.00 \\
.00 \\
5.80 \\
.00 \\
.00\end{array}$ & $\begin{array}{r}.00 \\
.00 \\
.56 \\
.00 \\
1.05\end{array}$ & $\begin{array}{l}.14 \\
.70 \\
.08 \\
.00 \\
.00\end{array}$ & $\begin{array}{r}.90 \\
.00 \\
.00 \\
.60 \\
1.00\end{array}$ & $\begin{array}{l}.00 \\
.00 \\
.07 \\
.00 \\
.00\end{array}$ \\
\hline $\begin{array}{l}26 \\
27 \\
28 \\
29 \\
30 \\
31\end{array}$ & $\begin{array}{l}.00 \\
.00 \\
.00 \\
.00 \\
.00 \\
.00\end{array}$ & $\begin{array}{l}.00 \\
.00 \\
.01 \\
.56 \\
.00 \\
.0-\end{array}$ & $\begin{array}{r}.00 \\
.00 \\
.14 \\
2.19 \\
.00 \\
.00\end{array}$ & $\begin{array}{l}.07 \\
.00 \\
.00 \\
.00 \\
.00 \\
.00\end{array}$ & $\begin{array}{l}.00 \\
.57 \\
.00 \\
.00 \\
-.- \\
.-\infty\end{array}$ & $\begin{array}{l}.13 \\
.40 \\
.01 \\
.00 \\
.00 \\
.00\end{array}$ & $\begin{array}{l}.00 \\
.00 \\
.00 \\
.00 \\
.00 \\
-.-\end{array}$ & $\begin{array}{l}.00 \\
.44 \\
.00 \\
.02 \\
.02 \\
.00\end{array}$ & $\begin{array}{r}.00 \\
.00 \\
.42 \\
1.00 \\
.65 \\
.--\end{array}$ & $\begin{array}{l}.05 \\
.04 \\
.00 \\
.00 \\
.36 \\
.20\end{array}$ & $\begin{array}{l}.00 \\
.00 \\
.00 \\
.00 \\
.00 \\
.00\end{array}$ & $\begin{array}{l}.00 \\
.46 \\
.00 \\
.00 \\
.00 \\
.0-\end{array}$ \\
\hline
\end{tabular}

Site 16--Daytona Brennan Water Plant

[Water year October 1984 to September 1985]

\begin{tabular}{|c|c|c|c|c|c|c|c|c|c|c|c|c|}
\hline $\begin{array}{l}1 \\
2 \\
3 \\
4 \\
5\end{array}$ & $\begin{array}{r}0.00 \\
.00 \\
.00 \\
.00 \\
.00\end{array}$ & $\begin{array}{r}0.00 \\
.03 \\
.07 \\
.00 \\
.00\end{array}$ & $\begin{array}{r}0.00 \\
.00 \\
.00 \\
.01 \\
.15\end{array}$ & $\begin{array}{r}0.00 \\
.00 \\
.70 \\
.00 \\
.00\end{array}$ & $\begin{array}{r}0.00 \\
.00 \\
.00 \\
.00 \\
.00\end{array}$ & $\begin{array}{r}0.00 \\
.00 \\
.00 \\
.00 \\
.00\end{array}$ & $\begin{array}{r}0.14 \\
.00 \\
.00 \\
.00 \\
.00\end{array}$ & $\begin{array}{r}0.00 \\
.00 \\
.00 \\
.64 \\
.00\end{array}$ & $\begin{array}{r}0.00 \\
.00 \\
.00 \\
.00 \\
.00\end{array}$ & $\begin{array}{r}0.00 \\
.00 \\
.00 \\
.00 \\
.79\end{array}$ & $\begin{array}{r}0.19 \\
.17 \\
.60 \\
.00 \\
3.70\end{array}$ & $\begin{array}{r}2.15 \\
.00 \\
.00 \\
.05 \\
.00\end{array}$ \\
\hline $\begin{array}{r}6 \\
7 \\
8 \\
9 \\
10\end{array}$ & $\begin{array}{l}.00 \\
.00 \\
.00 \\
.00 \\
.12\end{array}$ & $\begin{array}{l}.00 \\
.00 \\
.00 \\
.00 \\
.00\end{array}$ & $\begin{array}{l}.00 \\
.00 \\
.00 \\
.00 \\
.00\end{array}$ & $\begin{array}{l}.00 \\
.00 \\
.00 \\
.00 \\
.00\end{array}$ & $\begin{array}{l}.15 \\
.11 \\
.06 \\
.00 \\
.00\end{array}$ & $\begin{array}{l}.00 \\
.00 \\
.00 \\
.00 \\
.00\end{array}$ & $\begin{array}{l}.52 \\
.14 \\
.00 \\
.00 \\
.00\end{array}$ & $\begin{array}{l}.00 \\
.00 \\
.00 \\
.00 \\
.00\end{array}$ & $\begin{array}{l}.00 \\
.00 \\
.50 \\
.00 \\
.00\end{array}$ & $\begin{array}{r}.11 \\
1.10 \\
.00 \\
.00 \\
.00\end{array}$ & $\begin{array}{r}.00 \\
3.66 \\
.68 \\
.64 \\
.16\end{array}$ & $\begin{array}{l}.00 \\
.00 \\
.00 \\
.00 \\
.00\end{array}$ \\
\hline $\begin{array}{l}11 \\
12 \\
13 \\
14 \\
15\end{array}$ & $\begin{array}{l}.05 \\
.00 \\
.00 \\
.00 \\
.00\end{array}$ & $\begin{array}{l}.00 \\
.00 \\
.00 \\
.00 \\
.00\end{array}$ & $\begin{array}{l}.00 \\
.00 \\
.00 \\
.00 \\
.00\end{array}$ & $\begin{array}{l}.00 \\
.00 \\
.00 \\
.00 \\
.00\end{array}$ & $\begin{array}{l}.12 \\
.05 \\
.00 \\
.00 \\
.00\end{array}$ & $\begin{array}{l}.00 \\
.00 \\
.00 \\
.00 \\
.00\end{array}$ & $\begin{array}{r}.00 \\
.07 \\
1.40 \\
.18 \\
.00\end{array}$ & $\begin{array}{l}.00 \\
.00 \\
.00 \\
.00 \\
.00\end{array}$ & $\begin{array}{r}.00 \\
.82 \\
.92 \\
.98 \\
1.75\end{array}$ & $\begin{array}{r}.70 \\
1.00 \\
.00 \\
.00 \\
.00\end{array}$ & $\begin{array}{l}.50 \\
.00 \\
.00 \\
.68 \\
.00\end{array}$ & $\begin{array}{l}.00 \\
.00 \\
.50 \\
.00 \\
.00\end{array}$ \\
\hline $\begin{array}{l}16 \\
17 \\
18 \\
19 \\
20\end{array}$ & $\begin{array}{l}.00 \\
.00 \\
.00 \\
.00 \\
.00\end{array}$ & $\begin{array}{l}.00 \\
.00 \\
.00 \\
.00 \\
.00\end{array}$ & $\begin{array}{l}.00 \\
.00 \\
.00 \\
.00 \\
.00\end{array}$ & $\begin{array}{l}.00 \\
.03 \\
.15 \\
.00 \\
.00\end{array}$ & $\begin{array}{l}.00 \\
.00 \\
.00 \\
.00 \\
.00\end{array}$ & $\begin{array}{l}.10 \\
.34 \\
.00 \\
.00 \\
.00\end{array}$ & $\begin{array}{l}.00 \\
.00 \\
.00 \\
.00 \\
.00\end{array}$ & $\begin{array}{r}.00 \\
.00 \\
.00 \\
.00 \\
1.30\end{array}$ & $\begin{array}{l}.27 \\
.00 \\
.00 \\
.00 \\
.01\end{array}$ & $\begin{array}{r}1.74 \\
.00 \\
.00 \\
.00 \\
.00\end{array}$ & $\begin{array}{r}.03 \\
.00 \\
1.08 \\
.30 \\
.30\end{array}$ & $\begin{array}{r}.01 \\
1.37 \\
.32 \\
.82 \\
1.75\end{array}$ \\
\hline $\begin{array}{l}21 \\
22 \\
23 \\
24 \\
25\end{array}$ & $\begin{array}{l}.32 \\
.00 \\
.00 \\
.00 \\
.01\end{array}$ & $\begin{array}{r}.30 \\
1.60 \\
2.00 \\
.00 \\
.00\end{array}$ & $\begin{array}{l}.00 \\
.00 \\
.00 \\
.00 \\
.00\end{array}$ & $\begin{array}{l}.00 \\
.00 \\
.00 \\
.00 \\
.00\end{array}$ & $\begin{array}{l}.00 \\
.00 \\
.00 \\
.00 \\
.00\end{array}$ & $\begin{array}{r}1.35 \\
.00 \\
.00 \\
.00 \\
.00\end{array}$ & $\begin{array}{l}.00 \\
.00 \\
.00 \\
.00 \\
.00\end{array}$ & $\begin{array}{l}.00 \\
.80 \\
.80 \\
.02 \\
.00\end{array}$ & $\begin{array}{l}.02 \\
.00 \\
.00 \\
.00 \\
.00\end{array}$ & $\begin{array}{l}.18 \\
.04 \\
.11 \\
.60 \\
.00\end{array}$ & $\begin{array}{r}.18 \\
1.15 \\
.00 \\
.05 \\
.00\end{array}$ & $\begin{array}{l}.95 \\
.00 \\
.00 \\
.00 \\
.00\end{array}$ \\
\hline $\begin{array}{l}26 \\
27 \\
28 \\
29 \\
30 \\
31\end{array}$ & $\begin{array}{l}.45 \\
.00 \\
.00 \\
.00 \\
.20 \\
.00\end{array}$ & $\begin{array}{l}.00 \\
.00 \\
.05 \\
.00 \\
.00 \\
.--\end{array}$ & $\begin{array}{r}.07 \\
1.50 \\
.00 \\
.00 \\
.00 \\
.00\end{array}$ & $\begin{array}{l}.00 \\
.00 \\
.00 \\
.00 \\
.00 \\
.00\end{array}$ & $\begin{array}{l}.00 \\
.00 \\
.00 \\
-\ldots \\
-\ldots\end{array}$ & $\begin{array}{l}.00 \\
.00 \\
.00 \\
.00 \\
.00 \\
.00\end{array}$ & $\begin{array}{l}.00 \\
.00 \\
.00 \\
.00 \\
.35 \\
.--\end{array}$ & $\begin{array}{l}.00 \\
.00 \\
.00 \\
.00 \\
.00 \\
.00\end{array}$ & $\begin{array}{l}.00 \\
.00 \\
.31 \\
.13 \\
.00 \\
-.-\end{array}$ & $\begin{array}{r}.00 \\
.00 \\
.00 \\
.00 \\
.00 \\
1.20\end{array}$ & $\begin{array}{r}.00 \\
1.03 \\
.00 \\
.22 \\
.00 \\
.65\end{array}$ & $\begin{array}{l}.00 \\
.19 \\
.00 \\
.46 \\
.27 \\
. .-\end{array}$ \\
\hline
\end{tabular}


APPENDIX I--Daily rainfall--Continued

[Rainfall in inches]

\begin{tabular}{|c|c|c|c|c|c|c|c|c|c|c|c|c|}
\hline Day & Oct & Hov & Dec & $\operatorname{Jan}$ & Feb & Mar & Apr & May & June & July & Aus & Sept \\
\hline \multicolumn{13}{|c|}{$\begin{array}{l}\text { Site 17--NOAA weather station } \\
\text { y year October } 1982 \text { to September } 1983\end{array}$} \\
\hline $\begin{array}{l}1 \\
2 \\
3 \\
4 \\
5\end{array}$ & $\begin{array}{r}1.24 \\
.00 \\
.00 \\
.00 \\
.06\end{array}$ & $\begin{array}{r}0.21 \\
.17 \\
.55 \\
.03 \\
.00\end{array}$ & $\begin{array}{l}0.00 \\
.00 \\
.00 \\
.00 \\
.00\end{array}$ & $\begin{array}{r}0.00 \\
.25 \\
.00 \\
.00 \\
.00\end{array}$ & $\begin{array}{r}0.00 \\
1.95 \\
.00 \\
.00 \\
.00\end{array}$ & $\begin{array}{r}0.12 \\
.00 \\
.00 \\
.00 \\
.00\end{array}$ & $\begin{array}{l}0.00 \\
.04 \\
.00 \\
.00 \\
.00\end{array}$ & $\begin{array}{l}0.00 \\
.00 \\
.00 \\
.22 \\
.00\end{array}$ & $\begin{array}{l}0.00 \\
.00 \\
.00 \\
.18 \\
.86\end{array}$ & $\begin{array}{l}0.06 \\
.00 \\
.00 \\
.00 \\
.00\end{array}$ & $\begin{array}{l}0.00 \\
.00 \\
.00 \\
.36 \\
.00\end{array}$ & $\begin{array}{r}2.30 \\
1.10 \\
.41 \\
.00 \\
.00\end{array}$ \\
\hline $\begin{array}{r}6 \\
7 \\
8 \\
9 \\
10\end{array}$ & $\begin{array}{l}.00 \\
.00 \\
.00 \\
.00 \\
.00\end{array}$ & $\begin{array}{l}.00 \\
.10 \\
.00 \\
.00 \\
.00\end{array}$ & $\begin{array}{l}.03 \\
.72 \\
.32 \\
.15 \\
.99\end{array}$ & $\begin{array}{l}.00 \\
.00 \\
.00 \\
.10 \\
.00\end{array}$ & $\begin{array}{l}.74 \\
.00 \\
.00 \\
.00 \\
.40\end{array}$ & $\begin{array}{r}.00 \\
1.50 \\
.01 \\
.00 \\
.00\end{array}$ & $\begin{array}{r}.00 \\
1.86 \\
.01 \\
.95 \\
.26\end{array}$ & $\begin{array}{l}.00 \\
.00 \\
.00 \\
.00 \\
.00\end{array}$ & $\begin{array}{r}.19 \\
2.24 \\
.14 \\
.00 \\
.00\end{array}$ & $\begin{array}{l}.01 \\
.00 \\
.00 \\
.00 \\
.00\end{array}$ & $\begin{array}{r}.21 \\
.35 \\
.02 \\
2.15 \\
.00\end{array}$ & $\begin{array}{l}.00 \\
.00 \\
.00 \\
.00 \\
.00\end{array}$ \\
\hline $\begin{array}{l}11 \\
12 \\
13 \\
14 \\
15\end{array}$ & $\begin{array}{l}.08 \\
.00 \\
.00 \\
.23 \\
.00\end{array}$ & $\begin{array}{l}.00 \\
.00 \\
.00 \\
.00 \\
.08\end{array}$ & $\begin{array}{l}.00 \\
.11 \\
.00 \\
.00 \\
.01\end{array}$ & $\begin{array}{l}.00 \\
.00 \\
.00 \\
.00 \\
.00\end{array}$ & $\begin{array}{r}.00 \\
.85 \\
1.50 \\
.00 \\
.00\end{array}$ & $\begin{array}{r}.00 \\
.00 \\
.00 \\
.00 \\
2.48\end{array}$ & $\begin{array}{r}.00 \\
.00 \\
.00 \\
.00 \\
1.28\end{array}$ & $\begin{array}{l}.00 \\
.00 \\
.00 \\
.00 \\
.00\end{array}$ & $\begin{array}{l}.10 \\
.83 \\
.04 \\
.00 \\
.00\end{array}$ & $\begin{array}{l}.00 \\
.00 \\
.00 \\
.00 \\
.00\end{array}$ & $\begin{array}{r}.00 \\
.67 \\
1.04 \\
1.56 \\
.00\end{array}$ & $\begin{array}{l}.00 \\
.00 \\
.00 \\
.06 \\
.00\end{array}$ \\
\hline $\begin{array}{l}16 \\
17 \\
18 \\
19 \\
20\end{array}$ & $\begin{array}{l}.00 \\
.00 \\
.00 \\
.06 \\
.00\end{array}$ & $\begin{array}{l}.02 \\
.00 \\
.00 \\
.02 \\
.00\end{array}$ & $\begin{array}{l}.14 \\
.00 \\
.00 \\
.00 \\
.00\end{array}$ & $\begin{array}{r}.00 \\
.00 \\
.00 \\
.00 \\
1.27\end{array}$ & $\begin{array}{l}.36 \\
.04 \\
.00 \\
.00 \\
.00\end{array}$ & $\begin{array}{r}.00 \\
1.54 \\
.02 \\
.00 \\
.00\end{array}$ & $\begin{array}{l}.00 \\
.00 \\
.63 \\
.00 \\
.00\end{array}$ & $\begin{array}{l}.00 \\
.00 \\
.00 \\
.04 \\
.00\end{array}$ & $\begin{array}{l}.00 \\
.00 \\
.00 \\
.00 \\
.00\end{array}$ & $\begin{array}{l}.00 \\
.00 \\
.00 \\
.00 \\
.00\end{array}$ & $\begin{array}{l}.01 \\
.00 \\
.00 \\
.00 \\
.00\end{array}$ & $\begin{array}{r}.03 \\
1.23 \\
.25 \\
.15 \\
1.13\end{array}$ \\
\hline $\begin{array}{l}21 \\
22 \\
23 \\
24 \\
25\end{array}$ & $\begin{array}{r}.00 \\
.00 \\
1.55 \\
.01 \\
.00\end{array}$ & $\begin{array}{l}.00 \\
.00 \\
.00 \\
.00 \\
.00\end{array}$ & $\begin{array}{l}.00 \\
.00 \\
.00 \\
.00 \\
.00\end{array}$ & $\begin{array}{l}.23 \\
.01 \\
.18 \\
.00 \\
.00\end{array}$ & $\begin{array}{l}.02 \\
.02 \\
.00 \\
.00 \\
.00\end{array}$ & $\begin{array}{l}.39 \\
.00 \\
.00 \\
.37 \\
.00\end{array}$ & $\begin{array}{r}.00 \\
.00 \\
1.14 \\
.00 \\
.00\end{array}$ & $\begin{array}{l}.00 \\
.00 \\
.00 \\
.00 \\
.06\end{array}$ & $\begin{array}{r}1.26 \\
.12 \\
.40 \\
.00 \\
.00\end{array}$ & $\begin{array}{l}.00 \\
.00 \\
.00 \\
.00 \\
.00\end{array}$ & $\begin{array}{l}.00 \\
.00 \\
.00 \\
.00 \\
.21\end{array}$ & $\begin{array}{r}.09 \\
.00 \\
.00 \\
1.68 \\
.01\end{array}$ \\
\hline $\begin{array}{l}26 \\
27 \\
28 \\
29 \\
30 \\
31\end{array}$ & $\begin{array}{l}.00 \\
.00 \\
.00 \\
.00 \\
.00 \\
.00\end{array}$ & $\begin{array}{l}.38 \\
.01 \\
.01 \\
.00 \\
.00 \\
.-1\end{array}$ & $\begin{array}{l}.00 \\
.06 \\
.00 \\
.00 \\
.00 \\
.00\end{array}$ & $\begin{array}{l}.00 \\
.47 \\
.00 \\
.00 \\
.00 \\
.00\end{array}$ & $\begin{array}{l}.00 \\
.01 \\
.07 \\
--- \\
--- \\
---\end{array}$ & $\begin{array}{l}.00 \\
.48 \\
.00 \\
.00 \\
.00 \\
.80\end{array}$ & $\begin{array}{l}.00 \\
.00 \\
.00 \\
.00 \\
.00 \\
-0-\end{array}$ & $\begin{array}{r}.00 \\
.00 \\
.00 \\
2.61 \\
.93 \\
.00\end{array}$ & $\begin{array}{l}.00 \\
.00 \\
.00 \\
.00 \\
.01 \\
-.0\end{array}$ & $\begin{array}{l}.57 \\
.00 \\
.00 \\
.49 \\
.04 \\
.75\end{array}$ & $\begin{array}{l}.00 \\
.00 \\
.00 \\
.24 \\
.00 \\
.00\end{array}$ & $\begin{array}{l}.06 \\
.00 \\
.00 \\
.08 \\
.00\end{array}$ \\
\hline
\end{tabular}

Site 17--NOAA weather station

[Water year October 1983 to September 1984

\begin{tabular}{|c|c|c|c|c|c|c|c|c|c|c|c|c|}
\hline $\begin{array}{l}1 \\
2 \\
3 \\
4 \\
5\end{array}$ & $\begin{array}{r}0.00 \\
.00 \\
.00 \\
.00 \\
.00\end{array}$ & $\begin{array}{r}2.01 \\
.14 \\
.00 \\
.00 \\
.16\end{array}$ & $\begin{array}{r}0.05 \\
.00 \\
.00 \\
.00 \\
.00\end{array}$ & $\begin{array}{r}0.00 \\
.00 \\
.00 \\
.00 \\
.00\end{array}$ & $\begin{array}{r}0.00 \\
.02 \\
.01 \\
.00 \\
.00\end{array}$ & $\begin{array}{r}0.00 \\
.00 \\
.00 \\
.00 \\
.00\end{array}$ & $\begin{array}{r}0.00 \\
.00 \\
3.08 \\
.68 \\
.00\end{array}$ & $\begin{array}{r}0.00 \\
.00 \\
.00 \\
.93 \\
.01\end{array}$ & $\begin{array}{r}0.00 \\
.00 \\
.00 \\
.00 \\
.00\end{array}$ & $\begin{array}{r}1.04 \\
.71 \\
.48 \\
.37 \\
.00\end{array}$ & $\begin{array}{r}0.06 \\
.00 \\
.00 \\
.00 \\
.00\end{array}$ & $\begin{array}{r}0.00 \\
.00 \\
.00 \\
.17 \\
1.10\end{array}$ \\
\hline $\begin{array}{r}6 \\
7 \\
8 \\
9 \\
10\end{array}$ & $\begin{array}{l}.00 \\
.00 \\
.18 \\
.05 \\
.14\end{array}$ & $\begin{array}{l}.00 \\
.01 \\
.00 \\
.01 \\
.00\end{array}$ & $\begin{array}{l}.00 \\
.00 \\
.00 \\
.00 \\
.00\end{array}$ & $\begin{array}{l}.00 \\
.00 \\
.00 \\
.00 \\
.18\end{array}$ & $\begin{array}{l}.00 \\
.00 \\
.00 \\
.00 \\
.00\end{array}$ & $\begin{array}{l}.00 \\
.02 \\
.00 \\
.00 \\
.00\end{array}$ & $\begin{array}{l}.00 \\
.00 \\
.00 \\
.17 \\
.00\end{array}$ & $\begin{array}{l}.00 \\
.00 \\
.00 \\
.02 \\
.00\end{array}$ & $\begin{array}{l}.00 \\
.00 \\
.00 \\
.00 \\
.00\end{array}$ & $\begin{array}{l}.00 \\
.02 \\
.00 \\
.00 \\
.00\end{array}$ & $\begin{array}{l}.00 \\
.00 \\
.00 \\
.00 \\
.00\end{array}$ & $\begin{array}{r}1.57 \\
.05 \\
.01 \\
2.49 \\
.20\end{array}$ \\
\hline $\begin{array}{l}11 \\
12 \\
13 \\
14 \\
15\end{array}$ & $\begin{array}{r}1.57 \\
.14 \\
.05 \\
.02 \\
2.48\end{array}$ & $\begin{array}{l}.02 \\
.00 \\
.00 \\
.00 \\
.34\end{array}$ & $\begin{array}{r}3.65 \\
1.57 \\
.00 \\
.03 \\
.15\end{array}$ & $\begin{array}{l}.00 \\
.00 \\
.07 \\
.00 \\
.00\end{array}$ & $\begin{array}{l}.00 \\
.27 \\
.85 \\
.00 \\
.00\end{array}$ & $\begin{array}{l}.00 \\
.12 \\
.01 \\
.00 \\
.00\end{array}$ & $\begin{array}{r}.00 \\
.00 \\
.00 \\
1.05 \\
.00\end{array}$ & $\begin{array}{l}.00 \\
.00 \\
.00 \\
.00 \\
.00\end{array}$ & $\begin{array}{l}.08 \\
.24 \\
.02 \\
.00 \\
.00\end{array}$ & $\begin{array}{l}.00 \\
.00 \\
.04 \\
.00 \\
.00\end{array}$ & $\begin{array}{l}.26 \\
.91 \\
.00 \\
.00 \\
.00\end{array}$ & $\begin{array}{l}.00 \\
.00 \\
.00 \\
.00 \\
.00\end{array}$ \\
\hline $\begin{array}{l}16 \\
17 \\
18 \\
19 \\
20\end{array}$ & $\begin{array}{r}1.36 \\
2.90 \\
.03 \\
.00 \\
.00\end{array}$ & $\begin{array}{l}.02 \\
.00 \\
.00 \\
.00 \\
.63\end{array}$ & $\begin{array}{r}.00 \\
.79 \\
.57 \\
.07 \\
2.51\end{array}$ & $\begin{array}{l}.00 \\
.00 \\
.12 \\
.26 \\
.45\end{array}$ & $\begin{array}{l}.00 \\
.00 \\
.00 \\
.00 \\
.00\end{array}$ & $\begin{array}{l}.00 \\
.00 \\
.08 \\
.00 \\
.56\end{array}$ & $\begin{array}{l}.00 \\
.05 \\
.00 \\
.00 \\
.00\end{array}$ & $\begin{array}{l}.00 \\
.00 \\
.00 \\
.00 \\
.00\end{array}$ & $\begin{array}{l}.00 \\
.01 \\
.00 \\
.00 \\
.00\end{array}$ & $\begin{array}{r}.00 \\
.01 \\
.01 \\
1.08 \\
.48\end{array}$ & $\begin{array}{l}.00 \\
.26 \\
.00 \\
.27 \\
.12\end{array}$ & $\begin{array}{l}.00 \\
.20 \\
.82 \\
.03 \\
.00\end{array}$ \\
\hline $\begin{array}{l}21 \\
22 \\
23 \\
24 \\
25\end{array}$ & $\begin{array}{r}.12 \\
1.02 \\
.03 \\
.00 \\
.00\end{array}$ & $\begin{array}{l}.00 \\
.00 \\
.00 \\
.17 \\
.00\end{array}$ & $\begin{array}{l}.61 \\
.00 \\
.00 \\
.00 \\
.00\end{array}$ & $\begin{array}{l}.30 \\
.01 \\
.00 \\
.00 \\
.07\end{array}$ & $\begin{array}{r}.30 \\
1.57 \\
.00 \\
.00 \\
.00\end{array}$ & $\begin{array}{l}.00 \\
.00 \\
.00 \\
.05 \\
.00\end{array}$ & $\begin{array}{l}.00 \\
.00 \\
.26 \\
.00 \\
.00\end{array}$ & $\begin{array}{r}.00 \\
.00 \\
4.02 \\
.06 \\
.00\end{array}$ & $\begin{array}{l}.03 \\
.12 \\
.36 \\
.00 \\
.55\end{array}$ & $\begin{array}{r}1.48 \\
.47 \\
.32 \\
.06 \\
.00\end{array}$ & $\begin{array}{r}.66 \\
.00 \\
.00 \\
.32 \\
1.16\end{array}$ & $\begin{array}{l}.00 \\
.00 \\
.03 \\
.00 \\
.00\end{array}$ \\
\hline $\begin{array}{l}26 \\
27 \\
28 \\
29 \\
30 \\
31\end{array}$ & $\begin{array}{l}.00 \\
.00 \\
.00 \\
.00 \\
.00 \\
.02\end{array}$ & $\begin{array}{l}.00 \\
.00 \\
.00 \\
.51 \\
.00 \\
-.\end{array}$ & $\begin{array}{r}.00 \\
.00 \\
.14 \\
1.84 \\
.00 \\
.00\end{array}$ & $\begin{array}{l}.00 \\
.00 \\
.00 \\
.00 \\
.00 \\
.00\end{array}$ & $\begin{array}{l}.00 \\
.42 \\
.00 \\
.00 \\
---\end{array}$ & $\begin{array}{l}.10 \\
.37 \\
.00 \\
.00 \\
.00 \\
.00\end{array}$ & $\begin{array}{l}.00 \\
.00 \\
.00 \\
.00 \\
.00 \\
-.-\end{array}$ & $\begin{array}{l}.77 \\
.08 \\
.00 \\
.00 \\
.13 \\
.00\end{array}$ & $\begin{array}{l}.00 \\
.00 \\
.43 \\
.23 \\
.77 \\
-.--\end{array}$ & $\begin{array}{l}.00 \\
.00 \\
.00 \\
.00 \\
.18 \\
.02\end{array}$ & $\begin{array}{l}.00 \\
.00 \\
.00 \\
.00 \\
.00 \\
.00\end{array}$ & $\begin{array}{r}.00 \\
4.06 \\
.00 \\
.00 \\
.00 \\
-\end{array}$ \\
\hline
\end{tabular}


APPENDIX I--Daily rainfall--Continued

[Rainfall in inches]

\begin{tabular}{|c|c|c|c|c|c|c|c|c|c|c|c|c|}
\hline Day & Oct & Nov & Dec & $\operatorname{San}$ & Feb & Mar & Apr & May & June & July & Aug & Sept \\
\hline \multicolumn{13}{|c|}{$\begin{array}{l}\text { Site } 17-\text {-NOA weather station } \\
\text { [Water year October } 1984 \text { to September } 1985\end{array}$} \\
\hline $\begin{array}{l}1 \\
2 \\
3 \\
4 \\
5\end{array}$ & $\begin{array}{r}0.06 \\
.00 \\
.00 \\
.00 \\
.00\end{array}$ & $\begin{array}{r}0.00 \\
.01 \\
.25 \\
.06 \\
.00\end{array}$ & $\begin{array}{r}0.00 \\
.00 \\
.00 \\
.02 \\
.05\end{array}$ & $\begin{array}{r}0.00 \\
.00 \\
.64 \\
.00 \\
.00\end{array}$ & $\begin{array}{r}0.00 \\
.00 \\
.01 \\
.00 \\
.00\end{array}$ & $\begin{array}{r}0.00 \\
.00 \\
.00 \\
.00 \\
.00\end{array}$ & $\begin{array}{r}0.11 \\
.00 \\
.00 \\
.00 \\
.00\end{array}$ & $\begin{array}{r}0.00 \\
.00 \\
.00 \\
.70 \\
.00\end{array}$ & $\begin{array}{r}0.00 \\
.00 \\
.00 \\
.00 \\
.00\end{array}$ & $\begin{array}{r}0.00 \\
.00 \\
.00 \\
.00 \\
.44\end{array}$ & $\begin{array}{r}1.06 \\
.00 \\
.65 \\
.00 \\
.00\end{array}$ & $\begin{array}{r}2.35 \\
.00 \\
.00 \\
.00 \\
.01\end{array}$ \\
\hline $\begin{array}{r}6 \\
7 \\
8 \\
9 \\
10\end{array}$ & $\begin{array}{l}.00 \\
.00 \\
.00 \\
.00 \\
.07\end{array}$ & $\begin{array}{l}.00 \\
.00 \\
.00 \\
.00 \\
.00\end{array}$ & $\begin{array}{l}.00 \\
.00 \\
.00 \\
.00 \\
.00\end{array}$ & $\begin{array}{l}.00 \\
.00 \\
.00 \\
.00 \\
.00\end{array}$ & $\begin{array}{l}.18 \\
.11 \\
.07 \\
.00 \\
.00\end{array}$ & $\begin{array}{l}.00 \\
.00 \\
.00 \\
.00 \\
.00\end{array}$ & $\begin{array}{l}.51 \\
.08 \\
.00 \\
.00 \\
.00\end{array}$ & $\begin{array}{l}.00 \\
.00 \\
.00 \\
.00 \\
.00\end{array}$ & $\begin{array}{l}.00 \\
.00 \\
.80 \\
.00 \\
.00\end{array}$ & $\begin{array}{l}.02 \\
.16 \\
.00 \\
.00 \\
.00\end{array}$ & $\begin{array}{r}.00 \\
1.17 \\
.47 \\
.55 \\
.06\end{array}$ & $\begin{array}{l}.47 \\
.00 \\
.00 \\
.00 \\
.00\end{array}$ \\
\hline $\begin{array}{l}11 \\
12 \\
13 \\
14 \\
15\end{array}$ & $\begin{array}{l}.32 \\
.00 \\
.00 \\
.00 \\
.00\end{array}$ & $\begin{array}{l}.00 \\
.00 \\
.00 \\
.00 \\
.00\end{array}$ & $\begin{array}{l}.00 \\
.00 \\
.00 \\
.00 \\
.00\end{array}$ & $\begin{array}{l}.00 \\
.00 \\
.00 \\
.00 \\
.00\end{array}$ & $\begin{array}{l}.17 \\
.03 \\
.00 \\
.00 \\
.01\end{array}$ & $\begin{array}{l}.00 \\
.00 \\
.00 \\
.00 \\
.00\end{array}$ & $\begin{array}{r}.07 \\
1.63 \\
.39 \\
.00 \\
.00\end{array}$ & $\begin{array}{l}.00 \\
.00 \\
.00 \\
.00 \\
.00\end{array}$ & $\begin{array}{r}.00 \\
.83 \\
.45 \\
.94 \\
1.92\end{array}$ & $\begin{array}{l}.03 \\
.34 \\
.00 \\
.00 \\
.00\end{array}$ & $\begin{array}{l}.08 \\
.01 \\
.06 \\
.35 \\
.09\end{array}$ & $\begin{array}{l}.00 \\
.00 \\
.47 \\
.00 \\
.00\end{array}$ \\
\hline $\begin{array}{l}16 \\
17 \\
18 \\
19 \\
20\end{array}$ & $\begin{array}{l}.00 \\
.00 \\
.00 \\
.00 \\
.00\end{array}$ & $\begin{array}{l}.00 \\
.00 \\
.00 \\
.00 \\
.00\end{array}$ & $\begin{array}{l}.00 \\
.00 \\
.00 \\
.00 \\
.00\end{array}$ & $\begin{array}{l}.00 \\
.02 \\
.13 \\
.00 \\
.00\end{array}$ & $\begin{array}{l}.00 \\
.00 \\
.00 \\
.00 \\
.00\end{array}$ & $\begin{array}{l}.02 \\
.28 \\
.00 \\
.00 \\
.00\end{array}$ & $\begin{array}{l}.00 \\
.00 \\
.00 \\
.00 \\
.00\end{array}$ & $\begin{array}{l}.00 \\
.00 \\
.00 \\
.00 \\
.97\end{array}$ & $\begin{array}{l}.21 \\
.00 \\
.00 \\
.00 \\
.02\end{array}$ & $\begin{array}{l}.38 \\
.00 \\
.00 \\
.00 \\
.00\end{array}$ & $\begin{array}{l}.26 \\
.15 \\
.28 \\
.72 \\
.80\end{array}$ & $\begin{array}{r}.01 \\
2.29 \\
.16 \\
.73 \\
1.63\end{array}$ \\
\hline $\begin{array}{l}21 \\
22 \\
23 \\
24 \\
25\end{array}$ & $\begin{array}{l}.35 \\
.03 \\
.07 \\
.00 \\
.01\end{array}$ & $\begin{array}{r}.21 \\
1.57 \\
1.37 \\
.00 \\
.02\end{array}$ & $\begin{array}{l}.00 \\
.00 \\
.00 \\
.00 \\
.00\end{array}$ & $\begin{array}{l}.00 \\
.00 \\
.00 \\
.00 \\
.00\end{array}$ & $\begin{array}{l}.00 \\
.00 \\
.00 \\
.00 \\
.00\end{array}$ & $\begin{array}{r}1.19 \\
.00 \\
.00 \\
.00 \\
.00\end{array}$ & $\begin{array}{l}.00 \\
.00 \\
.00 \\
.00 \\
.00\end{array}$ & $\begin{array}{l}.00 \\
.95 \\
.80 \\
.00 \\
.00\end{array}$ & $\begin{array}{l}.51 \\
.00 \\
.00 \\
.05 \\
.11\end{array}$ & $\begin{array}{l}.02 \\
.00 \\
.01 \\
.33 \\
.41\end{array}$ & $\begin{array}{l}.00 \\
.79 \\
.00 \\
.22 \\
.00\end{array}$ & $\begin{array}{r}1.96 \\
.00 \\
.00 \\
.00 \\
.00\end{array}$ \\
\hline $\begin{array}{l}26 \\
27 \\
28 \\
29 \\
30 \\
31\end{array}$ & $\begin{array}{l}.17 \\
.00 \\
.00 \\
.00 \\
.01 \\
.00\end{array}$ & $\begin{array}{l}.00 \\
.00 \\
.03 \\
.00 \\
.00 \\
---\end{array}$ & $\begin{array}{l}.05 \\
.08 \\
.00 \\
.00 \\
.00 \\
.00\end{array}$ & $\begin{array}{l}.00 \\
.00 \\
.00 \\
.00 \\
.00 \\
.00\end{array}$ & \begin{tabular}{l}
.00 \\
.00 \\
.00 \\
\hdashline- \\
-- \\
--
\end{tabular} & $\begin{array}{l}.00 \\
.00 \\
.00 \\
.00 \\
.00 \\
.00\end{array}$ & $\begin{array}{l}.00 \\
.00 \\
.00 \\
.00 \\
.35 \\
.\end{array}$ & $\begin{array}{l}.00 \\
.00 \\
.00 \\
.00 \\
.00 \\
.00\end{array}$ & $\begin{array}{l}.00 \\
.00 \\
.68 \\
.03 \\
.26 \\
---\end{array}$ & $\begin{array}{l}.00 \\
.00 \\
.00 \\
.00 \\
.00 \\
.02\end{array}$ & $\begin{array}{l}.00 \\
.87 \\
.00 \\
.17 \\
.26 \\
.76\end{array}$ & $\begin{array}{l}.00 \\
.40 \\
.02 \\
.01 \\
.11 \\
.\end{array}$ \\
\hline
\end{tabular}

Site 18--Civil Defense Center

[Water year October 1982 to September 1983

\begin{tabular}{|c|c|c|c|c|c|c|c|c|c|c|c|c|}
\hline $\begin{array}{l}1 \\
2 \\
3 \\
4 \\
5\end{array}$ & $\begin{array}{l}-- \\
-- \\
--- \\
--\end{array}$ & $\begin{array}{l}--- \\
--- \\
--- \\
---\end{array}$ & $\begin{array}{l}--- \\
--- \\
--- \\
---\end{array}$ & $\begin{array}{l}--- \\
-- \\
-- \\
-- \\
-\end{array}$ & $\begin{array}{l}--- \\
--- \\
--- \\
---\end{array}$ & $\begin{array}{r}0.00 \\
.00 \\
.00 \\
.00 \\
.00\end{array}$ & $\begin{array}{r}0.00 \\
.09 \\
.00 \\
.00 \\
.00\end{array}$ & $\begin{array}{r}0.00 \\
.00 \\
.00 \\
.26 \\
.01\end{array}$ & $\begin{array}{r}0.00 \\
.00 \\
.00 \\
.53 \\
.49\end{array}$ & $\begin{array}{r}0.07 \\
.02 \\
.01 \\
.00 \\
.03\end{array}$ & $\begin{array}{r}0.00 \\
.11 \\
.01 \\
.16 \\
.02\end{array}$ & $\begin{array}{r}0.69 \\
.88 \\
.67 \\
.00 \\
.00\end{array}$ \\
\hline $\begin{array}{r}6 \\
7 \\
8 \\
9 \\
10\end{array}$ & $\begin{array}{l}-- \\
--- \\
--- \\
--\end{array}$ & $\begin{array}{l}--- \\
--- \\
--- \\
---\end{array}$ & $\begin{array}{l}--- \\
--- \\
--- \\
---\end{array}$ & $\begin{array}{l}--- \\
-- \\
--- \\
---\end{array}$ &. & $\begin{array}{r}.00 \\
1.93 \\
.01 \\
1.38 \\
.00\end{array}$ & $\begin{array}{r}.00 \\
2.09 \\
.13 \\
.00 \\
.00\end{array}$ & $\begin{array}{r}.00 \\
.00 \\
.00 \\
.00 \\
.00\end{array}$ & $\begin{array}{r}.60 \\
1.24 \\
.25 \\
.00 \\
.01\end{array}$ & $\begin{array}{r}.03 \\
.00 \\
.00 \\
.33 \\
.00\end{array}$ & $\begin{array}{r}.76 \\
.04 \\
.00 \\
.00 \\
.57\end{array}$ & $\begin{array}{l}.00 \\
.00 \\
.00 \\
.00\end{array}$ \\
\hline $\begin{array}{l}11 \\
12 \\
13 \\
14 \\
15\end{array}$ & $\begin{array}{l}--- \\
-- \\
--- \\
---\end{array}$ & $\begin{array}{l}--- \\
--- \\
--- \\
---\end{array}$ & $\begin{array}{l}--- \\
--- \\
--- \\
---\end{array}$ & $\begin{array}{l}--- \\
--- \\
--- \\
---\end{array}$ & $\begin{array}{l}--- \\
--- \\
--- \\
---\end{array}$ & $\begin{array}{r}.00 \\
.00 \\
.00 \\
.00 \\
1.66\end{array}$ & $\begin{array}{r}.00 \\
.00 \\
.00 \\
3.00 \\
.00\end{array}$ & $\begin{array}{l}.01 \\
.00 \\
.00 \\
.00 \\
.00\end{array}$ & $\begin{array}{r}.00 \\
.00 \\
1.28 \\
.05 \\
.00\end{array}$ & $\begin{array}{l}.00 \\
.00 \\
.00 \\
.00 \\
.00\end{array}$ & $\begin{array}{r}.00 \\
1.45 \\
.59 \\
2.13 \\
.00\end{array}$ & $\begin{array}{l}.00 \\
.00 \\
.01 \\
.11 \\
.00\end{array}$ \\
\hline $\begin{array}{l}16 \\
17 \\
18 \\
19 \\
20\end{array}$ & $\begin{array}{l}--- \\
--- \\
--- \\
--\end{array}$ & $\begin{array}{l}--- \\
--- \\
--- \\
---\end{array}$ & $\begin{array}{l}--- \\
--- \\
--- \\
---\end{array}$ & $\begin{array}{l}--- \\
--- \\
--- \\
---\end{array}$ & $\begin{array}{l}--- \\
--- \\
--- \\
---\end{array}$ & $\begin{array}{r}.01 \\
1.64 \\
.00 \\
.00 \\
.01\end{array}$ & $\begin{array}{l}.84 \\
.00 \\
.00 \\
.00 \\
.00\end{array}$ & $\begin{array}{l}.00 \\
.00 \\
.00 \\
.18 \\
.00\end{array}$ & $\begin{array}{l}.00 \\
.01 \\
.00 \\
.00 \\
.00\end{array}$ & $\begin{array}{l}.00 \\
.00 \\
.00 \\
.35 \\
.00\end{array}$ & $\begin{array}{l}.13 \\
.34 \\
.00 \\
.00 \\
.00\end{array}$ & $\begin{array}{r}1.98 \\
.54 \\
.02 \\
.26 \\
.30\end{array}$ \\
\hline $\begin{array}{l}21 \\
22 \\
23 \\
24 \\
25\end{array}$ & $\begin{array}{l}--- \\
-- \\
--- \\
---\end{array}$ & $\begin{array}{l}--- \\
--- \\
--- \\
---\end{array}$ & $\begin{array}{l}--- \\
--- \\
--- \\
---\end{array}$ & $\begin{array}{l}--- \\
--- \\
--- \\
---\end{array}$ & $\begin{array}{l}--- \\
-- \\
-- \\
---\end{array}$ & $\begin{array}{l}.20 \\
.00 \\
.00 \\
.38 \\
.00\end{array}$ & $\begin{array}{r}.00 \\
.00 \\
1.59 \\
.00 \\
.00\end{array}$ & $\begin{array}{l}.00 \\
.00 \\
.00 \\
.00 \\
.37\end{array}$ & $\begin{array}{r}2.10 \\
.32 \\
.81 \\
.01 \\
.00\end{array}$ & $\begin{array}{l}.01 \\
.00 \\
.00 \\
.00 \\
.00\end{array}$ & $\begin{array}{l}.00 \\
.00 \\
.00 \\
.00 \\
.23\end{array}$ & $\begin{array}{l}.08 \\
.03 \\
.00 \\
.92 \\
.49\end{array}$ \\
\hline $\begin{array}{l}26 \\
27 \\
28 \\
29 \\
30 \\
31\end{array}$ & $\begin{array}{l}--- \\
--- \\
--- \\
--- \\
---\end{array}$ & $\begin{array}{l}--- \\
--- \\
--- \\
--- \\
---\end{array}$ & $\begin{array}{l}--- \\
--- \\
--- \\
--- \\
--- \\
--\end{array}$ & $\begin{array}{l}--- \\
--- \\
--- \\
--- \\
---\end{array}$ & $\begin{array}{l}--- \\
-- \\
-- \\
--- \\
---\end{array}$ & $\begin{array}{l}.00 \\
.57 \\
.01 \\
.00 \\
.00 \\
.92\end{array}$ & $\begin{array}{l}.00 \\
.00 \\
.00 \\
.00 \\
.00 \\
.0\end{array}$ & $\begin{array}{r}.00 \\
.00 \\
.00 \\
.59 \\
1.02 \\
.00\end{array}$ & $\begin{array}{r}.00 \\
.00 \\
.00 \\
1.52 \\
.21 \\
.0\end{array}$ & $\begin{array}{r}1.00 \\
.00 \\
.01 \\
.36 \\
.08 \\
1.94\end{array}$ & $\begin{array}{l}.00 \\
.01 \\
.00 \\
.00 \\
.00 \\
.00\end{array}$ & $\begin{array}{l}.03 \\
.03 \\
.00 \\
.00 \\
.01 \\
.0\end{array}$ \\
\hline
\end{tabular}




\begin{tabular}{|c|c|c|c|c|c|c|c|c|c|c|c|c|}
\hline Day & Oct & Nov & Dec & Jan & Feb & $\operatorname{Mar}$ & Apr & May & June & July & Aus & Sept \\
\hline \multicolumn{13}{|c|}{$\begin{array}{l}\text { Site 18--Civil Defense Center } \\
\text { I year October } 1983 \text { to September } 1984\end{array}$} \\
\hline $\begin{array}{l}1 \\
2 \\
3 \\
4 \\
5\end{array}$ & $\begin{array}{l}0.00 \\
.00 \\
.00 \\
.00 \\
.00\end{array}$ & $\begin{array}{l}0.05 \\
.00 \\
.01 \\
.00 \\
.19\end{array}$ & $\begin{array}{l}0.00 \\
.00 \\
.00 \\
.06 \\
.01\end{array}$ & $\begin{array}{r}0.00 \\
.00 \\
.00 \\
.00 \\
.00\end{array}$ & $\begin{array}{l}--- \\
--- \\
--- \\
---\end{array}$ & $\begin{array}{l}0.00 \\
.00 \\
.00 \\
.00 \\
.00\end{array}$ & $\begin{array}{r}0.00 \\
.00 \\
2.85 \\
.69 \\
.00\end{array}$ & $\begin{array}{r}0.00 \\
.00 \\
.00 \\
.49 \\
.06\end{array}$ & $\begin{array}{l}0.00 \\
.00 \\
.00 \\
.00 \\
.00\end{array}$ & $\begin{array}{r}1.11 \\
.71 \\
1.18 \\
.00 \\
.01\end{array}$ & $\begin{array}{l}0.27 \\
.00 \\
.00 \\
.00 \\
.00\end{array}$ & $\begin{array}{r}0.00 \\
.00 \\
.00 \\
1.28 \\
1.65\end{array}$ \\
\hline $\begin{array}{r}6 \\
7 \\
8 \\
9 \\
10\end{array}$ & $\begin{array}{l}.00 \\
.00 \\
.01 \\
.24 \\
.08\end{array}$ & $\begin{array}{l}.00 \\
.05 \\
.02 \\
.95 \\
.02\end{array}$ & $\begin{array}{l}.02 \\
.00 \\
.00 \\
.00 \\
.00\end{array}$ & $\begin{array}{l}.00 \\
.00 \\
.00 \\
.00 \\
.21\end{array}$ & $\begin{array}{l}--- \\
.00 \\
.00 \\
.00\end{array}$ & $\begin{array}{l}.00 \\
.04 \\
.00 \\
.00 \\
.00\end{array}$ & $\begin{array}{l}.00 \\
.00 \\
.00 \\
.24 \\
.01\end{array}$ & $\begin{array}{l}.00 \\
.00 \\
.00 \\
.05 \\
.00\end{array}$ & $\begin{array}{l}.00 \\
.00 \\
.00 \\
.00 \\
.00\end{array}$ & $\begin{array}{l}.00 \\
.10 \\
.00 \\
.00 \\
.00\end{array}$ & $\begin{array}{l}.00 \\
.00 \\
.00 \\
.00 \\
.00\end{array}$ & $\begin{array}{r}1.47 \\
.42 \\
.05 \\
2.12 \\
.18\end{array}$ \\
\hline $\begin{array}{l}11 \\
12 \\
13 \\
14 \\
15\end{array}$ & $\begin{array}{r}.29 \\
.02 \\
.08 \\
.00 \\
1.38\end{array}$ & $\begin{array}{l}.05 \\
.00 \\
.00 \\
.00 \\
.53\end{array}$ & $\begin{array}{r}1.98 \\
2.59 \\
.00 \\
.02 \\
.15\end{array}$ & $\begin{array}{l}.00 \\
.00 \\
.09 \\
-.-- \\
.--\end{array}$ & $\begin{array}{r}.00 \\
.61 \\
1.35 \\
.01 \\
.00\end{array}$ & $\begin{array}{l}.00 \\
.05 \\
.00 \\
.00 \\
.00\end{array}$ & $\begin{array}{l}.00 \\
.00 \\
.00 \\
.96 \\
.00\end{array}$ & $\begin{array}{l}.00 \\
.00 \\
.01 \\
.00 \\
.00\end{array}$ & $\begin{array}{l}.11 \\
.02 \\
.53 \\
.01 \\
.00\end{array}$ & $\begin{array}{l}.00 \\
.00 \\
.41 \\
.00 \\
.02\end{array}$ & $\begin{array}{r}.00 \\
1.57 \\
.03 \\
.00 \\
.00\end{array}$ & $\begin{array}{l}.00 \\
.00 \\
.00 \\
.00 \\
.00\end{array}$ \\
\hline $\begin{array}{l}16 \\
17 \\
18 \\
19 \\
20\end{array}$ & $\begin{array}{r}2.25 \\
1.39 \\
.07 \\
.01 \\
.00\end{array}$ & $\begin{array}{l}.11 \\
.00 \\
.00 \\
.00 \\
.87\end{array}$ & $\begin{array}{r}.00 \\
.25 \\
.92 \\
.09 \\
1.29\end{array}$ & $\begin{array}{l}--- \\
--- \\
--- \\
--- \\
---\end{array}$ & $\begin{array}{l}.00 \\
.00 \\
.00 \\
.00 \\
.00\end{array}$ & $\begin{array}{l}.00 \\
.00 \\
.02 \\
.00 \\
.56\end{array}$ & $\begin{array}{l}.00 \\
.00 \\
.00 \\
.00 \\
.00\end{array}$ & $\begin{array}{l}.00 \\
.00 \\
.00 \\
.00 \\
.00\end{array}$ & $\begin{array}{l}.00 \\
.13 \\
.05 \\
.01 \\
.00\end{array}$ & $\begin{array}{r}.01 \\
.00 \\
.02 \\
1.72 \\
.46\end{array}$ & $\begin{array}{l}.00 \\
.06 \\
.36 \\
.93 \\
.22\end{array}$ & $\begin{array}{r}.00 \\
.32 \\
1.55 \\
.06 \\
.11\end{array}$ \\
\hline $\begin{array}{l}21 \\
22 \\
23 \\
24 \\
25\end{array}$ & $\begin{array}{r}.02 \\
1.06 \\
.02 \\
.00 \\
.01\end{array}$ & $\begin{array}{l}.00 \\
.00 \\
.00 \\
.16 \\
.00\end{array}$ & $\begin{array}{l}.32 \\
.04 \\
.00 \\
.00 \\
.00\end{array}$ & $\begin{array}{l}-=- \\
--- \\
--- \\
--- \\
---\end{array}$ & $\begin{array}{r}.54 \\
1.66 \\
.00 \\
.00 \\
.00\end{array}$ & $\begin{array}{l}.00 \\
.04 \\
.00 \\
.17 \\
.00\end{array}$ & $\begin{array}{l}.00 \\
.00 \\
.25 \\
.00 \\
.00\end{array}$ & $\begin{array}{r}.00 \\
.02 \\
3.84 \\
.32 \\
.01\end{array}$ & $\begin{array}{l}.07 \\
.29 \\
.26 \\
.00 \\
.84\end{array}$ & $\begin{array}{l}.26 \\
.79 \\
.08 \\
.01 \\
.00\end{array}$ & $\begin{array}{r}.45 \\
.01 \\
.00 \\
.67 \\
1.79\end{array}$ & $\begin{array}{l}.00 \\
.00 \\
.02 \\
.00 \\
.00\end{array}$ \\
\hline $\begin{array}{l}26 \\
27 \\
28 \\
29 \\
30 \\
31\end{array}$ & $\begin{array}{l}.00 \\
.01 \\
.00 \\
.01 \\
.01 \\
.23\end{array}$ & $\begin{array}{l}.00 \\
.00 \\
.02 \\
.48 \\
.00 \\
. .-\end{array}$ & $\begin{array}{r}.00 \\
.00 \\
.03 \\
2.19 \\
.01 \\
.01\end{array}$ & $\begin{array}{l}--- \\
--- \\
--- \\
--- \\
--- \\
--\end{array}$ & $\begin{array}{l}.00 \\
.47 \\
.04 \\
.00 \\
. .- \\
.--\end{array}$ & $\begin{array}{l}.11 \\
.44 \\
.00 \\
.00 \\
.00 \\
.00\end{array}$ & $\begin{array}{l}.00 \\
.00 \\
.00 \\
.00 \\
.00 \\
-.-\end{array}$ & $\begin{array}{l}.64 \\
.16 \\
.01 \\
.03 \\
.01 \\
.01\end{array}$ & $\begin{array}{l}.02 \\
.35 \\
.45 \\
.21 \\
.54 \\
.--\end{array}$ & $\begin{array}{l}.00 \\
.01 \\
.00 \\
.00 \\
.78 \\
.80\end{array}$ & $\begin{array}{l}.00 \\
.00 \\
.00 \\
.00 \\
.18 \\
.00\end{array}$ & $\begin{array}{r}.00 \\
1.84 \\
.00 \\
.00 \\
.00 \\
----\end{array}$ \\
\hline
\end{tabular}

Site 18--Civil Defense Center

[Water year October 1984 to September 1985

\begin{tabular}{|c|c|c|c|c|c|c|c|c|c|c|c|c|}
\hline $\begin{array}{l}1 \\
2 \\
3 \\
4 \\
5\end{array}$ & $\begin{array}{r}0.00 \\
.00 \\
.00 \\
.00 \\
.00\end{array}$ & $\begin{array}{r}0.00 \\
.02 \\
.50 \\
.09 \\
.00\end{array}$ & $\begin{array}{r}0.00 \\
.00 \\
.00 \\
.04 \\
.13\end{array}$ & $\begin{array}{r}0.00 \\
.00 \\
.50 \\
.02 \\
.00\end{array}$ & $\begin{array}{r}0.00 \\
.01 \\
.01 \\
.00 \\
.00\end{array}$ & $\begin{array}{r}0.00 \\
.00 \\
.00 \\
.00 \\
.00\end{array}$ & $\begin{array}{r}0.14 \\
.01 \\
.00 \\
.00 \\
.00\end{array}$ & $\begin{array}{r}0.00 \\
.00 \\
.00 \\
.32 \\
.00\end{array}$ & $\begin{array}{r}0.00 \\
.00 \\
.00 \\
.00 \\
.00\end{array}$ & $\begin{array}{r}0.02 \\
.00 \\
.00 \\
.00 \\
.63\end{array}$ & $\begin{array}{r}0.73 \\
.00 \\
.66 \\
.02 \\
.00\end{array}$ & $\begin{array}{r}2.49 \\
.00 \\
.01 \\
.46 \\
.05\end{array}$ \\
\hline $\begin{array}{r}6 \\
7 \\
8 \\
9 \\
10\end{array}$ & $\begin{array}{l}.00 \\
.00 \\
.00 \\
.00 \\
.00\end{array}$ & $\begin{array}{l}.00 \\
.00 \\
.00 \\
.00 \\
.00\end{array}$ & $\begin{array}{l}.01 \\
.00 \\
.00 \\
.00 \\
.00\end{array}$ & $\begin{array}{l}.00 \\
.00 \\
.00 \\
.00 \\
.00\end{array}$ & $\begin{array}{l}.17 \\
.02 \\
.02 \\
.00 \\
.00\end{array}$ & $\begin{array}{l}.00 \\
.00 \\
.00 \\
.00 \\
.00\end{array}$ & $\begin{array}{l}.45 \\
.02 \\
.00 \\
.02 \\
.00\end{array}$ & $\begin{array}{l}.00 \\
.00 \\
.00 \\
.00 \\
.00\end{array}$ & $\begin{array}{l}.00 \\
.02 \\
.67 \\
.00 \\
.00\end{array}$ & $\begin{array}{l}.06 \\
.67 \\
.01 \\
.00 \\
.00\end{array}$ & $\begin{array}{r}.01 \\
2.57 \\
.69 \\
.38 \\
.03\end{array}$ & $\begin{array}{l}.00 \\
.00 \\
.00 \\
.00 \\
.00\end{array}$ \\
\hline $\begin{array}{l}11 \\
12 \\
13 \\
14 \\
15\end{array}$ & $\begin{array}{l}.01 \\
.00 \\
.00 \\
.00 \\
.00\end{array}$ & $\begin{array}{l}.00 \\
.00 \\
.00 \\
.00 \\
.01\end{array}$ & $\begin{array}{l}.00 \\
.00 \\
.00 \\
.00 \\
.00\end{array}$ & $\begin{array}{l}.00 \\
.00 \\
.00 \\
.00 \\
.00\end{array}$ & $\begin{array}{l}.19 \\
.00 \\
.00 \\
.00 \\
.02\end{array}$ & $\begin{array}{l}.00 \\
.00 \\
.00 \\
.00 \\
.00\end{array}$ & $\begin{array}{l}.00 \\
.06 \\
.44 \\
.27 \\
.01\end{array}$ & $\begin{array}{l}.00 \\
.00 \\
.00 \\
.00 \\
.00\end{array}$ & $\begin{array}{r}.01 \\
1.39 \\
.87 \\
.71 \\
.81\end{array}$ & $\begin{array}{l}.42 \\
.76 \\
.01 \\
.00 \\
.16\end{array}$ & $\begin{array}{r}.02 \\
.02 \\
.94 \\
1.28 \\
.17\end{array}$ & $\begin{array}{l}.00 \\
.00 \\
.18 \\
.14 \\
.01\end{array}$ \\
\hline $\begin{array}{l}16 \\
17 \\
18 \\
19 \\
20\end{array}$ & $\begin{array}{l}.00 \\
.00 \\
.00 \\
.00 \\
.00\end{array}$ & $\begin{array}{l}.00 \\
.00 \\
.00 \\
.00 \\
.00\end{array}$ & $\begin{array}{l}.00 \\
.00 \\
.00 \\
.00 \\
.02\end{array}$ & $\begin{array}{l}.00 \\
.04 \\
.11 \\
.00 \\
.00\end{array}$ & $\begin{array}{l}.00 \\
.00 \\
.00 \\
.00 \\
.00\end{array}$ & $\begin{array}{l}.10 \\
.31 \\
.00 \\
.00 \\
.00\end{array}$ & $\begin{array}{l}.01 \\
.01 \\
.02 \\
.01 \\
.00\end{array}$ & $\begin{array}{r}.00 \\
.00 \\
.00 \\
.00 \\
1.41\end{array}$ & $\begin{array}{l}.07 \\
.02 \\
.00 \\
.00 \\
.00\end{array}$ & $\begin{array}{l}.17 \\
.18 \\
.00 \\
.27 \\
.01\end{array}$ & $\begin{array}{r}.00 \\
.87 \\
1.49 \\
2.29 \\
.10\end{array}$ & $\begin{array}{r}.05 \\
.99 \\
.31 \\
1.20 \\
2.15\end{array}$ \\
\hline $\begin{array}{l}21 \\
22 \\
23 \\
24 \\
25\end{array}$ & $\begin{array}{l}.00 \\
.00 \\
.12 \\
.00 \\
.00\end{array}$ & $\begin{array}{r}.09 \\
1.39 \\
1.89 \\
.00 \\
.02\end{array}$ & $\begin{array}{l}.00 \\
.00 \\
.00 \\
.00 \\
.00\end{array}$ & $\begin{array}{l}.00 \\
.00 \\
.00 \\
.00 \\
.00\end{array}$ & $\begin{array}{l}.00 \\
.00 \\
.00 \\
.00 \\
.00\end{array}$ & $\begin{array}{l}.43 \\
.05 \\
.00 \\
.00 \\
.00\end{array}$ & $\begin{array}{l}.00 \\
.00 \\
.00 \\
.00 \\
.00\end{array}$ & $\begin{array}{l}.00 \\
.78 \\
.67 \\
.06 \\
.00\end{array}$ & $\begin{array}{l}.02 \\
.00 \\
.01 \\
.00 \\
.00\end{array}$ & $\begin{array}{l}.03 \\
.14 \\
.05 \\
.19 \\
.21\end{array}$ & $\begin{array}{l}.00 \\
.03 \\
.00 \\
.28 \\
.00\end{array}$ & $\begin{array}{l}.58 \\
.00 \\
.00 \\
.00 \\
.00\end{array}$ \\
\hline $\begin{array}{l}26 \\
27 \\
28 \\
29 \\
30 \\
31\end{array}$ & $\begin{array}{r}.18 \\
.02 \\
.00 \\
.00 \\
1.62 \\
.01\end{array}$ & $\begin{array}{l}.00 \\
.01 \\
.04 \\
.00 \\
.00 \\
-.0-\end{array}$ & $\begin{array}{l}.00 \\
.09 \\
.00 \\
.02 \\
.00 \\
.00\end{array}$ & $\begin{array}{l}.00 \\
.00 \\
.00 \\
.00 \\
.00 \\
.00\end{array}$ & $\begin{array}{l}.01 \\
.00 \\
.00 \\
-- \\
-- \\
--\end{array}$ & $\begin{array}{l}.00 \\
.00 \\
.00 \\
.00 \\
.00 \\
.00\end{array}$ & $\begin{array}{l}.00 \\
.00 \\
.00 \\
.00 \\
.73 \\
-.-\end{array}$ & $\begin{array}{l}.00 \\
.00 \\
.00 \\
.00 \\
.00 \\
.00\end{array}$ & $\begin{array}{r}.21 \\
.01 \\
1.84 \\
.17 \\
.50 \\
-\end{array}$ & $\begin{array}{l}.00 \\
.00 \\
.02 \\
.00 \\
.00 \\
.08\end{array}$ & $\begin{array}{l}.00 \\
.72 \\
.01 \\
.37 \\
.70 \\
.62\end{array}$ & $\begin{array}{l}.00 \\
.00 \\
.45 \\
.16 \\
.10 \\
-.-\end{array}$ \\
\hline
\end{tabular}




\begin{tabular}{|c|c|c|c|c|c|c|c|c|c|c|c|c|}
\hline Day & Oct & Yov & DeC & $\operatorname{Tan}$ & Feb & Mar & Apr & May & June & July & Aug & Sept \\
\hline & \multicolumn{12}{|c|}{$\begin{array}{l}\text { Site 19--Port Orange Water Plant } \\
\text { [Water year October } 1982 \text { to September 1983] }\end{array}$} \\
\hline $\begin{array}{l}1 \\
2 \\
3 \\
4 \\
5\end{array}$ & $\begin{array}{r}0.75 \\
1.50 \\
.00 \\
.00 \\
.00\end{array}$ & $\begin{array}{r}0.00 \\
.00 \\
.00 \\
.30 \\
.10\end{array}$ & $\begin{array}{r}0.00 \\
.00 \\
.00 \\
.00 \\
.00\end{array}$ & $\begin{array}{r}0.00 \\
.00 \\
.00 \\
.01 \\
.00\end{array}$ & $\begin{array}{r}0.00 \\
1.10 \\
.06 \\
.06 \\
.00\end{array}$ & $\begin{array}{r}0.02 \\
.06 \\
.00 \\
.00 \\
.00\end{array}$ & $\begin{array}{r}0.00 \\
.00 \\
.01 \\
.00 \\
.00\end{array}$ & $\begin{array}{r}0.00 \\
.00 \\
.00 \\
.00 \\
.00\end{array}$ & $\begin{array}{r}0.00 \\
.00 \\
.00 \\
.00 \\
.10\end{array}$ & $\begin{array}{r}0.00 \\
.00 \\
.70 \\
.00 \\
.00\end{array}$ & $\begin{array}{r}0.14 \\
.00 \\
.00 \\
.00 \\
.75\end{array}$ & $\begin{array}{r}0.40 \\
1.10 \\
.43 \\
.16 \\
.00\end{array}$ \\
\hline $\begin{array}{r}6 \\
7 \\
8 \\
9 \\
10\end{array}$ & $\begin{array}{l}.10 \\
.00 \\
.00 \\
.00 \\
.00\end{array}$ & $\begin{array}{l}.00 \\
.00 \\
.30 \\
.00 \\
.00\end{array}$ & $\begin{array}{r}.00 \\
1.50 \\
.60 \\
.10 \\
.60\end{array}$ & $\begin{array}{l}.00 \\
.00 \\
.00 \\
.00 \\
.17\end{array}$ & $\begin{array}{l}.50 \\
.73 \\
.00 \\
.00 \\
.00\end{array}$ & $\begin{array}{r}.00 \\
1.10 \\
.50 \\
.00 \\
.01\end{array}$ & $\begin{array}{l}.00 \\
.00 \\
.87 \\
.00 \\
.00\end{array}$ & $\begin{array}{l}.00 \\
.00 \\
.00 \\
.00 \\
.00\end{array}$ & $\begin{array}{r}.85 \\
.14 \\
1.27 \\
.05 \\
.00\end{array}$ & $\begin{array}{l}.00 \\
.20 \\
.31 \\
.00 \\
.00\end{array}$ & $\begin{array}{r}.00 \\
.21 \\
.30 \\
.20 \\
1.00\end{array}$ & $\begin{array}{l}.00 \\
.00 \\
.00 \\
.00 \\
.00\end{array}$ \\
\hline $\begin{array}{l}11 \\
12 \\
13 \\
14 \\
15\end{array}$ & $\begin{array}{l}.00 \\
.20 \\
.00 \\
.00 \\
.50\end{array}$ & $\begin{array}{l}.00 \\
.00 \\
.00 \\
.00 \\
.10\end{array}$ & $\begin{array}{l}.30 \\
.02 \\
.03 \\
.00 \\
.01\end{array}$ & $\begin{array}{l}.01 \\
.00 \\
.00 \\
.00 \\
.00\end{array}$ & $\begin{array}{r}.00 \\
.00 \\
2.50 \\
.33 \\
.00\end{array}$ & $\begin{array}{l}.00 \\
.00 \\
.00 \\
.00 \\
.05\end{array}$ & $\begin{array}{l}.01 \\
.00 \\
.00 \\
.00 \\
.00\end{array}$ & $\begin{array}{l}.00 \\
.90 \\
.32 \\
.00 \\
.00\end{array}$ & $\begin{array}{l}.00 \\
.00 \\
.07 \\
.26 \\
.00\end{array}$ & $\begin{array}{l}.00 \\
.00 \\
.00 \\
.00 \\
.00\end{array}$ & $\begin{array}{r}1.75 \\
.00 \\
.64 \\
.20 \\
1.82\end{array}$ & $\begin{array}{l}.00 \\
.00 \\
.00 \\
.00 \\
.11\end{array}$ \\
\hline $\begin{array}{l}16 \\
17 \\
18 \\
19 \\
20\end{array}$ & $\begin{array}{l}.00 \\
.00 \\
.00 \\
.00 \\
.00\end{array}$ & $\begin{array}{l}.00 \\
.00 \\
.00 \\
.00 \\
.00\end{array}$ & $\begin{array}{l}.00 \\
.20 \\
.00 \\
.00 \\
.00\end{array}$ & $\begin{array}{l}.00 \\
.00 \\
.00 \\
.00 \\
.42\end{array}$ & $\begin{array}{l}.00 \\
.47 \\
.01 \\
.00 \\
.00\end{array}$ & $\begin{array}{r}2.65 \\
.70 \\
.10 \\
.00 \\
.00\end{array}$ & $\begin{array}{r}2.10 \\
.00 \\
.00 \\
.50 \\
.01\end{array}$ & $\begin{array}{l}.00 \\
.00 \\
.00 \\
.06 \\
.01\end{array}$ & $\begin{array}{l}.00 \\
.00 \\
.00 \\
.00 \\
.00\end{array}$ & $\begin{array}{l}.00 \\
.00 \\
.00 \\
.00 \\
.90\end{array}$ & $\begin{array}{l}.00 \\
.50 \\
.00 \\
.00 \\
.00\end{array}$ & $\begin{array}{r}.00 \\
.00 \\
1.91 \\
.16 \\
.40\end{array}$ \\
\hline $\begin{array}{l}21 \\
22 \\
23 \\
24 \\
25\end{array}$ & $\begin{array}{r}.00 \\
.20 \\
.30 \\
1.50 \\
.00\end{array}$ & $\begin{array}{l}.00 \\
.00 \\
.00 \\
.00 \\
.00\end{array}$ & $\begin{array}{l}.00 \\
.00 \\
.00 \\
.00 \\
.01\end{array}$ & $\begin{array}{r}1.16 \\
.00 \\
.50 \\
.02 \\
.00\end{array}$ & $\begin{array}{l}.00 \\
.20 \\
.00 \\
.00 \\
.00\end{array}$ & $\begin{array}{l}.82 \\
.00 \\
.00 \\
.51 \\
.01\end{array}$ & $\begin{array}{r}.00 \\
.00 \\
.10 \\
1.92 \\
.00\end{array}$ & $\begin{array}{l}.00 \\
.00 \\
.00 \\
.00 \\
.00\end{array}$ & $\begin{array}{r}.02 \\
1.40 \\
.15 \\
.52 \\
.00\end{array}$ & $\begin{array}{l}.00 \\
.00 \\
.00 \\
.00 \\
.00\end{array}$ & $\begin{array}{l}.00 \\
.00 \\
.00 \\
.00 \\
.50\end{array}$ & $\begin{array}{l}.30 \\
.12 \\
.00 \\
.00 \\
.97\end{array}$ \\
\hline $\begin{array}{l}26 \\
27 \\
28 \\
29 \\
30 \\
31\end{array}$ & $\begin{array}{l}.00 \\
.00 \\
.00 \\
.00 \\
.00 \\
.00\end{array}$ & $\begin{array}{l}.00 \\
.50 \\
.00 \\
.00 \\
.00 \\
.--\end{array}$ & $\begin{array}{l}.00 \\
.05 \\
.00 \\
.00 \\
.00 \\
.00\end{array}$ & $\begin{array}{l}.00 \\
.00 \\
.00 \\
.00 \\
.00 \\
.00\end{array}$ & $\begin{array}{l}.00 \\
.00 \\
.07 \\
-- \\
-- \\
--\end{array}$ & $\begin{array}{l}.00 \\
.50 \\
.45 \\
.00 \\
.00 \\
.72\end{array}$ & $\begin{array}{l}.00 \\
.00 \\
.00 \\
.00 \\
.00 \\
.0\end{array}$ & $\begin{array}{r}.45 \\
.16 \\
.00 \\
.00 \\
1.68 \\
.75\end{array}$ & $\begin{array}{l}.10 \\
.00 \\
.00 \\
.00 \\
.00 \\
-.-\end{array}$ & $\begin{array}{l}.00 \\
.82 \\
.25 \\
.35 \\
.12 \\
.32\end{array}$ & $\begin{array}{r}1.30 \\
.00 \\
.00 \\
.20 \\
.00 \\
.00\end{array}$ & $\begin{array}{l}.05 \\
.00 \\
.00 \\
.01 \\
.20 \\
---\end{array}$ \\
\hline
\end{tabular}

Site 19--Port Orange Water Plant

[Water year October 1983 to September 1984]

\begin{tabular}{|c|c|c|c|c|c|c|c|c|c|c|c|}
\hline $\begin{array}{r}0.00 \\
.00 \\
.00 \\
.00 \\
.00\end{array}$ & $\begin{array}{r}0.02 \\
.04 \\
.00 \\
.00 \\
.10\end{array}$ & $\begin{array}{r}0.00 \\
.00 \\
.00 \\
.00 \\
.00\end{array}$ & $\begin{array}{r}0.00 \\
.00 \\
.00 \\
.00 \\
.00\end{array}$ & $\begin{array}{r}0.00 \\
.00 \\
.03 \\
.00 \\
.00\end{array}$ & $\begin{array}{r}0.00 \\
.00 \\
.00 \\
.00 \\
.00\end{array}$ & $\begin{array}{r}0.00 \\
.00 \\
.00 \\
.98 \\
.10\end{array}$ & $\begin{array}{r}0.00 \\
.00 \\
.00 \\
.00 \\
.96\end{array}$ & $\begin{array}{r}0.00 \\
.00 \\
.00 \\
.00 \\
.00\end{array}$ & $\begin{array}{r}1.30 \\
.40 \\
.33 \\
.60 \\
.04\end{array}$ & $\begin{array}{r}0.07 \\
.60 \\
.00 \\
.00 \\
.00\end{array}$ & $\begin{array}{r}0.00 \\
.00 \\
.00 \\
.00 \\
.18\end{array}$ \\
\hline $\begin{array}{r}.00 \\
.00 \\
.00 \\
1.03 \\
.19\end{array}$ & $\begin{array}{l}.09 \\
.00 \\
.03 \\
.00 \\
.00\end{array}$ & $\begin{array}{l}.07 \\
.17 \\
.00 \\
.00 \\
.00\end{array}$ & $\begin{array}{l}.00 \\
.00 \\
.00 \\
.00 \\
.15\end{array}$ & $\begin{array}{l}.00 \\
.00 \\
.00 \\
.00 \\
.00\end{array}$ & $\begin{array}{l}.00 \\
.10 \\
.00 \\
.00 \\
.00\end{array}$ & $\begin{array}{l}.00 \\
.00 \\
.00 \\
.06 \\
.26\end{array}$ & $\begin{array}{l}.06 \\
.00 \\
.00 \\
.10 \\
.00\end{array}$ & $\begin{array}{l}.00 \\
.00 \\
.00 \\
.00 \\
.00\end{array}$ & $\begin{array}{l}.00 \\
.00 \\
.00 \\
.00 \\
.00\end{array}$ & $\begin{array}{l}.00 \\
.00 \\
.00 \\
.00 \\
.00\end{array}$ & $\begin{array}{r}.52 \\
1.63 \\
.10 \\
.70 \\
2.32\end{array}$ \\
\hline $\begin{array}{r}.03 \\
2.35 \\
.12 \\
.15 \\
.65\end{array}$ & $\begin{array}{l}.00 \\
.00 \\
.00 \\
.00 \\
.00\end{array}$ & $\begin{array}{l}.00 \\
4.30 \\
.00 \\
.00 \\
.30\end{array}$ & $\begin{array}{l}.07 \\
.00 \\
.10 \\
.02 \\
.01\end{array}$ & $\begin{array}{r}.00 \\
.00 \\
.36 \\
1.10 \\
.00\end{array}$ & $\begin{array}{l}.00 \\
.00 \\
.19 \\
.00 \\
.00\end{array}$ & $\begin{array}{l}.00 \\
.00 \\
.04 \\
.00 \\
.86\end{array}$ & $\begin{array}{l}.00 \\
.00 \\
.00 \\
.00 \\
.00\end{array}$ & $\begin{array}{l}.15 \\
.07 \\
.16 \\
.03 \\
.00\end{array}$ & $\begin{array}{l}.00 \\
.00 \\
.16 \\
.08 \\
.00\end{array}$ & $\begin{array}{l}.00 \\
.60 \\
.17 \\
.00 \\
.00\end{array}$ & $\begin{array}{l}.00 \\
.10 \\
.00 \\
.00 \\
.00\end{array}$ \\
\hline $\begin{array}{r}4.10 \\
1.11 \\
.00 \\
.48 \\
.00\end{array}$ & $\begin{array}{l}.50 \\
.00 \\
.00 \\
.00 \\
.00\end{array}$ & $\begin{array}{l}.03 \\
.02 \\
.96 \\
.06 \\
.45\end{array}$ & $\begin{array}{l}.00 \\
.00 \\
.03 \\
.34 \\
.00\end{array}$ & $\begin{array}{l}.00 \\
.00 \\
.00 \\
.00 \\
.00\end{array}$ & $\begin{array}{l}.00 \\
.00 \\
.07 \\
.00 \\
.00\end{array}$ & $\begin{array}{l}.00 \\
.00 \\
.02 \\
.00 \\
.00\end{array}$ & $\begin{array}{l}.00 \\
.00 \\
.00 \\
.00 \\
.00\end{array}$ & $\begin{array}{l}.00 \\
.00 \\
.00 \\
.38 \\
.00\end{array}$ & $\begin{array}{l}.00 \\
.00 \\
.03 \\
.00 \\
.85\end{array}$ & $\begin{array}{r}.00 \\
.00 \\
.24 \\
.91 \\
1.09\end{array}$ & $\begin{array}{r}.00 \\
.00 \\
.01 \\
1.15 \\
.40\end{array}$ \\
\hline $\begin{array}{r}.00 \\
1.12 \\
.08 \\
.00 \\
.00\end{array}$ & $\begin{array}{l}.90 \\
.00 \\
.00 \\
.00 \\
.15\end{array}$ & $\begin{array}{r}.98 \\
1.00 \\
.00 \\
.00 \\
.00\end{array}$ & $\begin{array}{r}1.06 \\
.00 \\
.03 \\
.00 \\
.00\end{array}$ & $\begin{array}{r}.06 \\
.88 \\
2.05 \\
.00 \\
.00\end{array}$ & $\begin{array}{l}.60 \\
.00 \\
.00 \\
.10 \\
.00\end{array}$ & $\begin{array}{l}.00 \\
.00 \\
.00 \\
.06 \\
.00\end{array}$ & $\begin{array}{r}.00 \\
.00 \\
1.92 \\
1.86 \\
.06\end{array}$ & $\begin{array}{r}.00 \\
.02 \\
.10 \\
1.00 \\
.00\end{array}$ & $\begin{array}{l}.64 \\
.41 \\
.46 \\
.25 \\
.00\end{array}$ & $\begin{array}{r}.50 \\
.99 \\
.00 \\
.18 \\
1.61\end{array}$ & .0 \\
\hline $\begin{array}{l}.00 \\
.00 \\
.00 \\
.00 \\
.00 \\
.16\end{array}$ & $\begin{array}{l}.00 \\
.00 \\
.00 \\
.38 \\
.02 \\
.-\end{array}$ & $\begin{array}{r}.00 \\
.00 \\
.00 \\
.40 \\
1.80 \\
.01\end{array}$ & $\begin{array}{l}.00 \\
.00 \\
.00 \\
.00 \\
.00 \\
.00\end{array}$ & $\begin{array}{l}.00 \\
.08 \\
.37 \\
.02 \\
-.-\end{array}$ & $\begin{array}{l}.06 \\
.27 \\
.25 \\
.00 \\
.00 \\
.00\end{array}$ & $\begin{array}{l}.00 \\
.00 \\
.00 \\
.00 \\
.00 \\
.0\end{array}$ & $\begin{array}{r}.61 \\
.38 \\
.00 \\
.00 \\
1.20 \\
.00\end{array}$ & $\begin{array}{r}1.60 \\
.00 \\
.00 \\
.45 \\
.55 \\
-.-\end{array}$ & $\begin{array}{l}.00 \\
.00 \\
.04 \\
.00 \\
.00 \\
.43\end{array}$ & $\begin{array}{l}.00 \\
.00 \\
.00 \\
.00 \\
.00 \\
.00\end{array}$ & $\begin{array}{r}.00 \\
.00 \\
3.40 \\
.00 \\
.00\end{array}$ \\
\hline
\end{tabular}




\begin{tabular}{|c|c|c|c|c|c|c|c|c|c|c|c|c|}
\hline Day & Oct & Nov & Dec & Jan & Feb & Mar & Apr & May & June & July & Aus & Sept \\
\hline & \multicolumn{12}{|c|}{$\begin{array}{l}\text { Site 19--Port Orange Water Plant } \\
\text { [Water year October } 1984 \text { to September 1985] }\end{array}$} \\
\hline $\begin{array}{l}1 \\
2 \\
3 \\
4 \\
5\end{array}$ & $\begin{array}{l}0.00 \\
.00 \\
.00 \\
.00 \\
.00\end{array}$ & $\begin{array}{l}0.00 \\
.00 \\
.23 \\
.24 \\
.01\end{array}$ & $\begin{array}{l}0.00 \\
.01 \\
.02 \\
.01 \\
.02\end{array}$ & $\begin{array}{r}0.00 \\
.00 \\
.00 \\
1.06 \\
.00\end{array}$ & $\begin{array}{l}0.00 \\
.00 \\
.01 \\
.00 \\
.00\end{array}$ & $\begin{array}{l}0.00 \\
.00 \\
.01 \\
.01 \\
.00\end{array}$ & $\begin{array}{r}0.00 \\
.10 \\
.00 \\
.00 \\
.00\end{array}$ & $\begin{array}{r}0.00 \\
.00 \\
.00 \\
.25 \\
.30\end{array}$ & $\begin{array}{r}0.10 \\
.00 \\
.00 \\
.00 \\
.00\end{array}$ & $\begin{array}{l}0.25 \\
.00 \\
.00 \\
.00 \\
.00\end{array}$ & $\begin{array}{r}0.42 \\
.32 \\
.33 \\
.88 \\
.00\end{array}$ & $\begin{array}{r}1.57 \\
1.09 \\
.00 \\
.00 \\
.10\end{array}$ \\
\hline $\begin{array}{r}6 \\
7 \\
8 \\
9 \\
10\end{array}$ & $\begin{array}{l}.00 \\
.00 \\
.03 \\
.01 \\
.00\end{array}$ & $\begin{array}{l}.00 \\
.00 \\
.00 \\
.00 \\
.00\end{array}$ & $\begin{array}{l}.02 \\
.00 \\
.00 \\
.00 \\
.00\end{array}$ & $\begin{array}{l}.00 \\
.00 \\
.00 \\
.00 \\
.00\end{array}$ & $\begin{array}{l}.00 \\
.14 \\
.15 \\
.00 \\
.00\end{array}$ & $\begin{array}{l}.00 \\
.07 \\
.00 \\
.00 \\
.00\end{array}$ & $\begin{array}{l}.00 \\
.82 \\
.00 \\
.00 \\
.00\end{array}$ & $\begin{array}{l}.00 \\
.00 \\
.00 \\
.00 \\
.00\end{array}$ & $\begin{array}{l}.00 \\
.00 \\
.00 \\
.11 \\
.00\end{array}$ & $\begin{array}{l}.90 \\
.00 \\
.23 \\
.00 \\
.00\end{array}$ & $\begin{array}{l}.00 \\
.24 \\
.77 \\
.55 \\
.31\end{array}$ & $\begin{array}{l}.01 \\
.00 \\
.00 \\
.00 \\
.00\end{array}$ \\
\hline $\begin{array}{l}11 \\
12 \\
13 \\
14 \\
15\end{array}$ & $\begin{array}{l}.55 \\
.00 \\
.00 \\
.00 \\
.00\end{array}$ & $\begin{array}{l}.00 \\
.00 \\
.00 \\
.00 \\
.00\end{array}$ & $\begin{array}{l}.00 \\
.00 \\
.00 \\
.00 \\
.00\end{array}$ & $\begin{array}{l}.00 \\
.00 \\
.00 \\
.00 \\
.01\end{array}$ & $\begin{array}{l}.00 \\
.22 \\
.00 \\
.00 \\
.03\end{array}$ & $\begin{array}{l}.00 \\
.00 \\
.00 \\
.00 \\
.00\end{array}$ & $\begin{array}{r}.00 \\
.00 \\
1.15 \\
.55 \\
.09\end{array}$ & $\begin{array}{l}.00 \\
.00 \\
.00 \\
.00 \\
.00\end{array}$ & $\begin{array}{r}.00 \\
.00 \\
1.68 \\
1.20 \\
2.57\end{array}$ & $\begin{array}{l}.00 \\
.08 \\
.40 \\
.00 \\
.00\end{array}$ & $\begin{array}{l}.02 \\
.39 \\
.50 \\
.73 \\
.92\end{array}$ & $\begin{array}{l}.00 \\
.00 \\
.00 \\
.23 \\
.04\end{array}$ \\
\hline $\begin{array}{l}16 \\
17 \\
18 \\
19 \\
20\end{array}$ & $\begin{array}{l}.00 \\
.00 \\
.00 \\
.00 \\
.00\end{array}$ & $\begin{array}{l}.00 \\
.00 \\
.00 \\
.00 \\
.00\end{array}$ & $\begin{array}{l}.01 \\
.01 \\
.02 \\
.00 \\
.00\end{array}$ & $\begin{array}{l}.00 \\
.00 \\
.04 \\
.15 \\
.00\end{array}$ & $\begin{array}{l}.00 \\
.00 \\
.00 \\
.00 \\
.00\end{array}$ & $\begin{array}{l}.00 \\
.20 \\
.12 \\
.00 \\
.00\end{array}$ & $\begin{array}{l}.00 \\
.00 \\
.00 \\
.00 \\
.00\end{array}$ & $\begin{array}{l}.00 \\
.00 \\
.10 \\
.00 \\
.00\end{array}$ & $\begin{array}{l}.03 \\
.06 \\
.00 \\
.00 \\
.00\end{array}$ & $\begin{array}{l}.00 \\
.02 \\
.01 \\
.00 \\
.00\end{array}$ & $\begin{array}{l}.10 \\
.45 \\
.01 \\
.07 \\
.74\end{array}$ & $\begin{array}{r}.00 \\
2.50 \\
.15 \\
.62 \\
1.80\end{array}$ \\
\hline $\begin{array}{l}21 \\
22 \\
23 \\
24 \\
00\end{array}$ & $\begin{array}{l}.00 \\
.42 \\
.20 \\
.04 \\
.00\end{array}$ & $\begin{array}{r}.00 \\
.65 \\
2.40 \\
1.16 \\
.02\end{array}$ & $\begin{array}{l}.00 \\
.10 \\
.00 \\
.01 \\
.00\end{array}$ & $\begin{array}{l}.00 \\
.00 \\
.00 \\
.00 \\
.00\end{array}$ & $\begin{array}{l}.00 \\
.00 \\
.00 \\
.00 \\
.00\end{array}$ & $\begin{array}{l}.01 \\
.96 \\
.00 \\
.00 \\
.00\end{array}$ & $\begin{array}{l}.00 \\
.01 \\
.00 \\
.00 \\
.00\end{array}$ & $\begin{array}{r}2.70 \\
.00 \\
.78 \\
.23 \\
.16\end{array}$ & $\begin{array}{l}.01 \\
.01 \\
.01 \\
.00 \\
.28\end{array}$ & $\begin{array}{l}.00 \\
.00 \\
.02 \\
.22 \\
.14\end{array}$ & $\begin{array}{r}3.13 \\
.00 \\
.40 \\
.00 \\
.49\end{array}$ & $\begin{array}{r}3.60 \\
.00 \\
.00 \\
.00 \\
.00\end{array}$ \\
\hline $\begin{array}{l}26 \\
27 \\
28 \\
29 \\
30 \\
31\end{array}$ & $\begin{array}{l}.28 \\
.62 \\
.00 \\
.00 \\
.00 \\
.00\end{array}$ & $\begin{array}{l}.00 \\
.00 \\
.03 \\
.04 \\
.00 \\
-.-\end{array}$ & $\begin{array}{l}.00 \\
.05 \\
.03 \\
.00 \\
.01 \\
.00\end{array}$ & $\begin{array}{l}.00 \\
.00 \\
.02 \\
.01 \\
.01 \\
.01\end{array}$ & $\begin{array}{l}.00 \\
.00 \\
.00 \\
--- \\
--- \\
---\end{array}$ & $\begin{array}{l}.00 \\
.00 \\
.00 \\
.00 \\
.00 \\
.00\end{array}$ & $\begin{array}{l}.00 \\
.00 \\
.00 \\
.01 \\
.16 \\
-.-\end{array}$ & $\begin{array}{l}.00 \\
.00 \\
.00 \\
.00 \\
.00 \\
.00\end{array}$ & $\begin{array}{l}.00 \\
.04 \\
.00 \\
.67 \\
.15 \\
-.-\end{array}$ & $\begin{array}{l}.23 \\
.00 \\
.00 \\
.02 \\
.00 \\
.00\end{array}$ & $\begin{array}{l}.01 \\
.75 \\
.27 \\
.10 \\
.15 \\
.18\end{array}$ & $\begin{array}{r}.00 \\
.00 \\
3.25 \\
.18 \\
.23 \\
---\end{array}$ \\
\hline
\end{tabular}

Site 20--Volusia County Landfill

[Water year October 1982 to September 1983]

\begin{tabular}{|c|c|c|c|c|c|c|c|c|c|c|c|c|}
\hline $\begin{array}{l}1 \\
2 \\
3 \\
4 \\
5\end{array}$ & $\begin{array}{r}0.40 \\
.80 \\
.00 \\
.00 \\
.00\end{array}$ & $\begin{array}{r}0.00 \\
.00 \\
.40 \\
.00 \\
.00\end{array}$ & $\begin{array}{r}0.00 \\
.00 \\
.00 \\
.00 \\
.00\end{array}$ & $\begin{array}{r}0.00 \\
.00 \\
.00 \\
.00 \\
.00\end{array}$ & $\begin{array}{r}0.00 \\
2.50 \\
.00 \\
.00 \\
.00\end{array}$ & $\begin{array}{r}0.00 \\
.00 \\
.00 \\
.00 \\
.00\end{array}$ & $\begin{array}{r}0.00 \\
.03 \\
.00 \\
.00 \\
.00\end{array}$ & $\begin{array}{r}0.00 \\
.00 \\
.00 \\
.00 \\
.40\end{array}$ & $\begin{array}{r}0.00 \\
.00 \\
.00 \\
.00 \\
.00\end{array}$ & $\begin{array}{r}0.09 \\
.00 \\
.00 \\
.00 \\
.00\end{array}$ & $\begin{array}{r}0.03 \\
.00 \\
.00 \\
.00 \\
.28\end{array}$ & $\begin{array}{r}0.29 \\
2.87 \\
.49 \\
.00 \\
.00\end{array}$ \\
\hline $\begin{array}{r}6 \\
7 \\
8 \\
9 \\
10\end{array}$ & $\begin{array}{l}.20 \\
.00 \\
.00 \\
.00 \\
.00\end{array}$ & $\begin{array}{l}.00 \\
.00 \\
.00 \\
.00 \\
.00\end{array}$ & $\begin{array}{l}.00 \\
.50 \\
.50 \\
.00 \\
.00\end{array}$ & $\begin{array}{l}.00 \\
.00 \\
.00 \\
.00 \\
.00\end{array}$ & $\begin{array}{r}1.50 \\
.00 \\
.00 \\
.00 \\
.00\end{array}$ & $\begin{array}{r}.00 \\
1.40 \\
.00 \\
.00 \\
.00\end{array}$ & $\begin{array}{r}.00 \\
1.17 \\
.00 \\
.00 \\
1.60\end{array}$ & $\begin{array}{l}.00 \\
.00 \\
.00 \\
.00 \\
.00\end{array}$ & $\begin{array}{r}.83 \\
.28 \\
1.22 \\
.06 \\
.00\end{array}$ & $\begin{array}{l}.00 \\
.00 \\
.00 \\
.00 \\
.00\end{array}$ & $\begin{array}{l}.40 \\
.15 \\
.02 \\
.00 \\
.64\end{array}$ & $\begin{array}{l}.00 \\
.00 \\
.00 \\
.00 \\
.00\end{array}$ \\
\hline $\begin{array}{l}11 \\
12 \\
13 \\
14 \\
15\end{array}$ & $\begin{array}{r}.00 \\
.70 \\
.00 \\
4.00 \\
.00\end{array}$ & $\begin{array}{l}.00 \\
.00 \\
.00 \\
.00 \\
.30\end{array}$ & $\begin{array}{l}.00 \\
.50 \\
.20 \\
.00 \\
.00\end{array}$ & $\begin{array}{l}.00 \\
.00 \\
.00 \\
.00 \\
.00\end{array}$ & $\begin{array}{r}.50 \\
.00 \\
2.00 \\
.70 \\
.00\end{array}$ & $\begin{array}{l}.00 \\
.00 \\
.00 \\
.00 \\
.00\end{array}$ & $\begin{array}{r}.00 \\
.00 \\
.00 \\
.00 \\
2.20\end{array}$ & $\begin{array}{l}.00 \\
.00 \\
.00 \\
.00 \\
.00\end{array}$ & $\begin{array}{l}.00 \\
.00 \\
.58 \\
.00 \\
.00\end{array}$ & $\begin{array}{l}.00 \\
.00 \\
.00 \\
.00 \\
.00\end{array}$ & $\begin{array}{r}.05 \\
.10 \\
.37 \\
4.47 \\
.00\end{array}$ & $\begin{array}{l}.00 \\
.00 \\
.00 \\
.00 \\
.06\end{array}$ \\
\hline $\begin{array}{l}16 \\
17 \\
18 \\
19 \\
20\end{array}$ & $\begin{array}{l}.00 \\
.00 \\
.00 \\
.00 \\
.00\end{array}$ & $\begin{array}{l}.00 \\
.00 \\
.00 \\
.00 \\
.00\end{array}$ & $\begin{array}{l}.00 \\
.00 \\
.00 \\
.00 \\
.00\end{array}$ & $\begin{array}{l}.00 \\
.00 \\
.00 \\
.00 \\
.30\end{array}$ & $\begin{array}{l}.00 \\
.00 \\
.00 \\
.00 \\
.00\end{array}$ & $\begin{array}{r}2.35 \\
1.41 \\
.21 \\
.00 \\
.00\end{array}$ & $\begin{array}{l}.00 \\
.00 \\
.65 \\
.00 \\
.00\end{array}$ & $\begin{array}{l}.00 \\
.00 \\
.00 \\
.00 \\
.16\end{array}$ & $\begin{array}{l}.00 \\
.00 \\
.00 \\
.00 \\
.00\end{array}$ & $\begin{array}{l}.00 \\
.00 \\
.00 \\
.26 \\
.00\end{array}$ & $\begin{array}{l}.70 \\
.10 \\
.00 \\
.00 \\
.00\end{array}$ & $\begin{array}{r}.00 \\
.30 \\
1.35 \\
.00 \\
.70\end{array}$ \\
\hline $\begin{array}{l}21 \\
22 \\
23 \\
24 \\
25\end{array}$ & $\begin{array}{l}.00 \\
.00 \\
.00 \\
.00 \\
.00\end{array}$ & $\begin{array}{l}.00 \\
.00 \\
.00 \\
.00 \\
.00\end{array}$ & $\begin{array}{l}.00 \\
.00 \\
.00 \\
.00 \\
.00\end{array}$ & $\begin{array}{r}1.40 \\
.00 \\
.00 \\
.00 \\
.00\end{array}$ & $\begin{array}{l}.00 \\
.00 \\
.00 \\
.00 \\
.00\end{array}$ & $\begin{array}{l}.80 \\
.00 \\
.00 \\
.00 \\
.55\end{array}$ & $\begin{array}{l}.00 \\
.00 \\
.00 \\
.10 \\
.00\end{array}$ & $\begin{array}{l}.00 \\
.00 \\
.00 \\
.00 \\
.00\end{array}$ & $\begin{array}{r}.00 \\
1.78 \\
.58 \\
.95 \\
.03\end{array}$ & $\begin{array}{r}2.80 \\
.00 \\
.00 \\
.00 \\
.00\end{array}$ & $\begin{array}{l}.00 \\
.00 \\
.00 \\
.00 \\
.00\end{array}$ & $\begin{array}{r}.60 \\
.22 \\
.00 \\
1.15 \\
.03\end{array}$ \\
\hline $\begin{array}{l}26 \\
27 \\
28 \\
29 \\
30 \\
31\end{array}$ & $\begin{array}{l}.00 \\
.00 \\
.00 \\
.00 \\
.00 \\
.00\end{array}$ & $\begin{array}{l}.00 \\
.00 \\
.00 \\
.00 \\
.00 \\
-.--\end{array}$ & $\begin{array}{l}.00 \\
.00 \\
.00 \\
.00 \\
.00 \\
.00\end{array}$ & $\begin{array}{l}.00 \\
.00 \\
.00 \\
.00 \\
.00 \\
.00\end{array}$ & $\begin{array}{l}.00 \\
.00 \\
.00 \\
--- \\
--- \\
--\end{array}$ & $\begin{array}{l}.00 \\
.00 \\
.63 \\
.00 \\
.00 \\
.73\end{array}$ & $\begin{array}{l}.00 \\
.00 \\
.00 \\
.00 \\
.00 \\
-.-\end{array}$ & $\begin{array}{r}.10 \\
.00 \\
.00 \\
.00 \\
.00 \\
2.40\end{array}$ & $\begin{array}{l}.00 \\
.00 \\
.00 \\
.00 \\
.00 \\
---\end{array}$ & $\begin{array}{l}.00 \\
.75 \\
.43 \\
.15 \\
.42 \\
.61\end{array}$ & $\begin{array}{l}.20 \\
.00 \\
.26 \\
.00 \\
.22 \\
.00\end{array}$ & $\begin{array}{l}.00 \\
.03 \\
.00 \\
.00 \\
.00\end{array}$ \\
\hline
\end{tabular}


APPENDIX I--Daily rainfall--Continued

[Rainfall in inches]

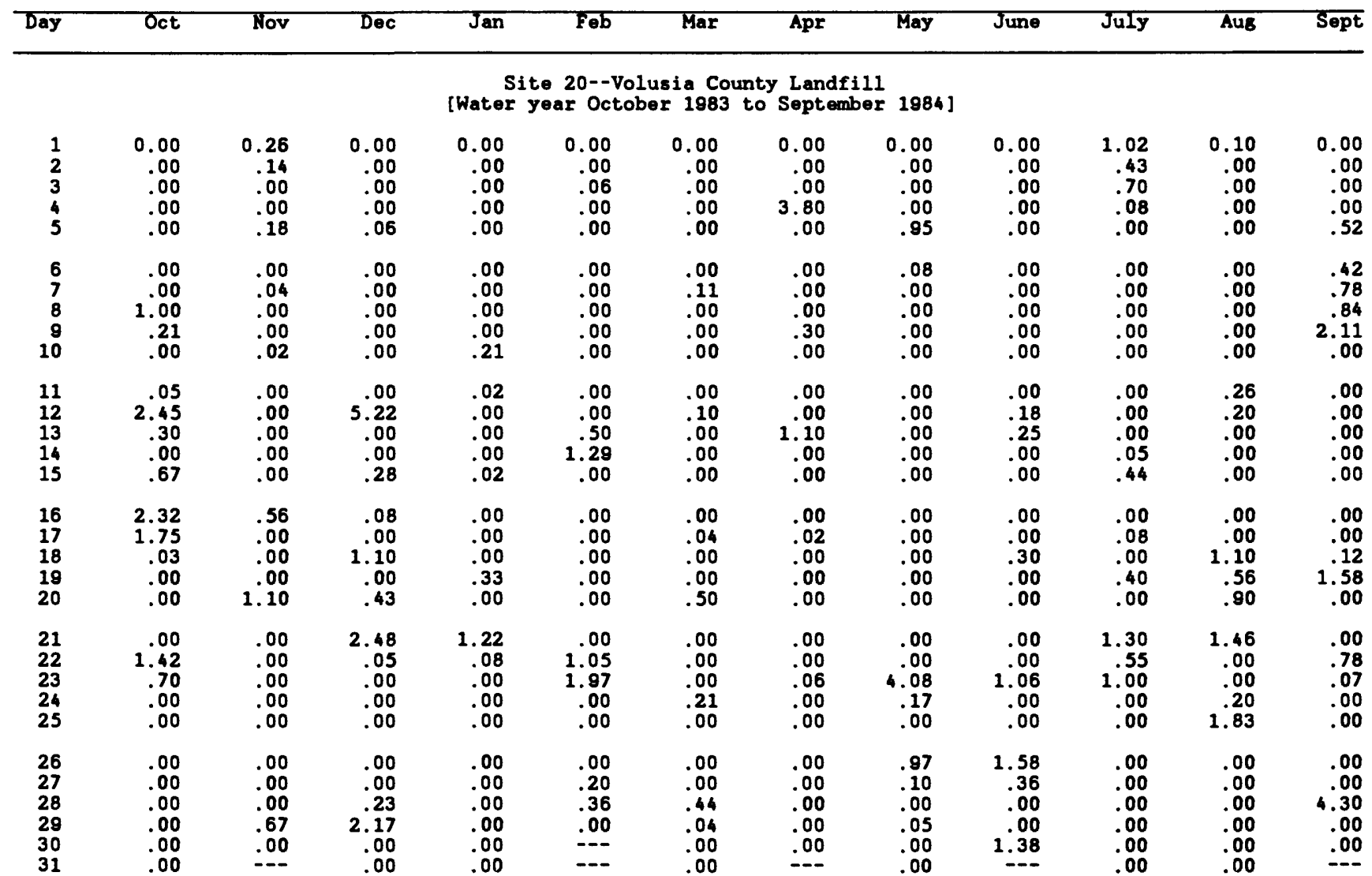

Site 20--Volusia County Landfill

[Water year October 1984 to September 1985]

\begin{tabular}{|c|c|c|c|c|c|c|c|c|c|c|c|c|}
\hline $\begin{array}{l}1 \\
2 \\
3 \\
4 \\
5\end{array}$ & $\begin{array}{l}0.00 \\
.00 \\
.00 \\
.00 \\
.00\end{array}$ & $\begin{array}{r}0.00 \\
.00 \\
.00 \\
.00 \\
.00\end{array}$ & $\begin{array}{r}0.00 \\
.00 \\
.00 \\
.00 \\
.00\end{array}$ & $\begin{array}{r}0.00 \\
.00 \\
.40 \\
.40 \\
.00\end{array}$ & $\begin{array}{r}0.00 \\
.00 \\
.00 \\
.20 \\
.00\end{array}$ & $\begin{array}{r}0.00 \\
.00 \\
.00 \\
.00 \\
.00\end{array}$ & $\begin{array}{r}0.00 \\
.00 \\
.00 \\
.00 \\
.00\end{array}$ & $\begin{array}{r}0.00 \\
.00 \\
.00 \\
.00 \\
.70\end{array}$ & $\begin{array}{r}0.00 \\
.00 \\
.00 \\
.00 \\
.00\end{array}$ & $\begin{array}{r}0.00 \\
.00 \\
.00 \\
.50 \\
.15\end{array}$ & $\begin{array}{r}2.10 \\
1.00 \\
2.20 \\
.00 \\
.00\end{array}$ & $\begin{array}{r}2.00 \\
.00 \\
.20 \\
.00 \\
.00\end{array}$ \\
\hline $\begin{array}{r}6 \\
7 \\
8 \\
9 \\
10\end{array}$ & $\begin{array}{l}.00 \\
.00 \\
.17 \\
.00 \\
.00\end{array}$ & $\begin{array}{l}.00 \\
.00 \\
.00 \\
.00 \\
.00\end{array}$ & $\begin{array}{l}.00 \\
.00 \\
.00 \\
.00 \\
.00\end{array}$ & $\begin{array}{l}.00 \\
.00 \\
.00 \\
.00 \\
.00\end{array}$ & $\begin{array}{l}.00 \\
.00 \\
.24 \\
.00 \\
.00\end{array}$ & $\begin{array}{r}1.00 \\
.10 \\
.00 \\
.00 \\
.00\end{array}$ & $\begin{array}{l}.00 \\
.00 \\
.00 \\
.00 \\
.00\end{array}$ & $\begin{array}{l}.00 \\
.00 \\
.00 \\
.00 \\
.00\end{array}$ & $\begin{array}{l}.00 \\
.00 \\
.85 \\
.00 \\
.00\end{array}$ & $\begin{array}{l}.00 \\
.50 \\
.00 \\
.00 \\
.00\end{array}$ & $\begin{array}{r}2.20 \\
2.30 \\
.60 \\
1.20 \\
.10\end{array}$ & $\begin{array}{l}.20 \\
.00 \\
.00 \\
.00 \\
.00\end{array}$ \\
\hline $\begin{array}{l}11 \\
12 \\
13 \\
14 \\
15\end{array}$ & $\begin{array}{l}.00 \\
.48 \\
.00 \\
.00 \\
.00\end{array}$ & $\begin{array}{l}.00 \\
.00 \\
.00 \\
.00 \\
.00\end{array}$ & $\begin{array}{l}.00 \\
.00 \\
.00 \\
.00 \\
.05\end{array}$ & $\begin{array}{l}.00 \\
.00 \\
.00 \\
.00 \\
.00\end{array}$ & $\begin{array}{l}.20 \\
.00 \\
.00 \\
.00 \\
.00\end{array}$ & $\begin{array}{l}.00 \\
.00 \\
.00 \\
.00 \\
.00\end{array}$ & $\begin{array}{r}.00 \\
.00 \\
1.50 \\
1.00 \\
.00\end{array}$ & $\begin{array}{l}.00 \\
.00 \\
.00 \\
.00 \\
.00\end{array}$ & $\begin{array}{r}.00 \\
.00 \\
2.00 \\
2.00 \\
2.20\end{array}$ & $\begin{array}{l}.00 \\
.00 \\
.00 \\
.00 \\
.60\end{array}$ & $\begin{array}{r}.00 \\
.90 \\
1.50 \\
1.50 \\
.10\end{array}$ & $\begin{array}{l}.00 \\
.05 \\
.12 \\
.10 \\
.00\end{array}$ \\
\hline $\begin{array}{l}16 \\
17 \\
18 \\
19 \\
20\end{array}$ & $\begin{array}{l}.00 \\
.00 \\
.00 \\
.00 \\
.00\end{array}$ & $\begin{array}{l}.00 \\
.00 \\
.00 \\
.00 \\
.00\end{array}$ & $\begin{array}{l}.00 \\
.00 \\
.00 \\
.00 \\
.00\end{array}$ & $\begin{array}{l}.00 \\
.00 \\
.00 \\
.19 \\
.00\end{array}$ & $\begin{array}{l}.00 \\
.00 \\
.00 \\
.00 \\
.00\end{array}$ & $\begin{array}{l}.00 \\
.27 \\
.00 \\
.00 \\
.00\end{array}$ & $\begin{array}{l}.00 \\
.00 \\
.00 \\
.00 \\
.00\end{array}$ & $\begin{array}{r}.00 \\
.00 \\
.00 \\
.00 \\
1.00\end{array}$ & $\begin{array}{l}.00 \\
.00 \\
.00 \\
.00 \\
.00\end{array}$ & $\begin{array}{l}.00 \\
.00 \\
.30 \\
.00 \\
.00\end{array}$ & $\begin{array}{r}.00 \\
.00 \\
.70 \\
1.25 \\
.55\end{array}$ & $\begin{array}{r}1.52 \\
.20 \\
.60 \\
1.78 \\
2.00\end{array}$ \\
\hline $\begin{array}{l}21 \\
22 \\
23 \\
24 \\
25\end{array}$ & $\begin{array}{l}.00 \\
.78 \\
.18 \\
.00 \\
.00\end{array}$ & $\begin{array}{r}.00 \\
.55 \\
1.55 \\
1.60 \\
.40\end{array}$ & $\begin{array}{l}.00 \\
.00 \\
.00 \\
.00 \\
.00\end{array}$ & $\begin{array}{l}.00 \\
.00 \\
.00 \\
.00 \\
.00\end{array}$ & $\begin{array}{l}.00 \\
.00 \\
.00 \\
.00 \\
.00\end{array}$ & $\begin{array}{l}.00 \\
.00 \\
.00 \\
.00 \\
.00\end{array}$ & $\begin{array}{l}.00 \\
.00 \\
.00 \\
.00 \\
.00\end{array}$ & $\begin{array}{r}.00 \\
.00 \\
1.00 \\
1.00 \\
.00\end{array}$ & $\begin{array}{l}.00 \\
.25 \\
.00 \\
.00 \\
.00\end{array}$ & $\begin{array}{l}.00 \\
.20 \\
.00 \\
.00 \\
.00\end{array}$ & $\begin{array}{r}.00 \\
1.00 \\
.00 \\
.00 \\
.40\end{array}$ & $\begin{array}{l}.50 \\
.00 \\
.00 \\
.00 \\
.00\end{array}$ \\
\hline $\begin{array}{l}26 \\
27 \\
28 \\
29 \\
30 \\
31\end{array}$ & $\begin{array}{l}.00 \\
.00 \\
.00 \\
.00 \\
.51 \\
.00\end{array}$ & $\begin{array}{l}.00 \\
.00 \\
.00 \\
.08 \\
.00 \\
.00\end{array}$ & $\begin{array}{l}.00 \\
.00 \\
.00 \\
.00 \\
.00 \\
.00\end{array}$ & $\begin{array}{l}.00 \\
.00 \\
.00 \\
.00 \\
.00 \\
.00\end{array}$ & \begin{tabular}{l}
.00 \\
.00 \\
.00 \\
\hdashline- \\
.- \\
--
\end{tabular} & $\begin{array}{l}.00 \\
.00 \\
.00 \\
.00 \\
.00 \\
.00\end{array}$ & $\begin{array}{l}.00 \\
.00 \\
.00 \\
.00 \\
.00 \\
-.\end{array}$ & $\begin{array}{l}.00 \\
.00 \\
.00 \\
.00 \\
.00 \\
.00\end{array}$ & $\begin{array}{r}.00 \\
.00 \\
.00 \\
1.00 \\
.65 \\
---\end{array}$ & $\begin{array}{r}.00 \\
.00 \\
1.40 \\
.00 \\
.00 \\
.00\end{array}$ & $\begin{array}{r}.15 \\
.75 \\
.20 \\
.00 \\
.45 \\
1.30\end{array}$ & $\begin{array}{r}.00 \\
1.00 \\
.55 \\
.00 \\
.35 \\
.-\end{array}$ \\
\hline
\end{tabular}


Appendix II 


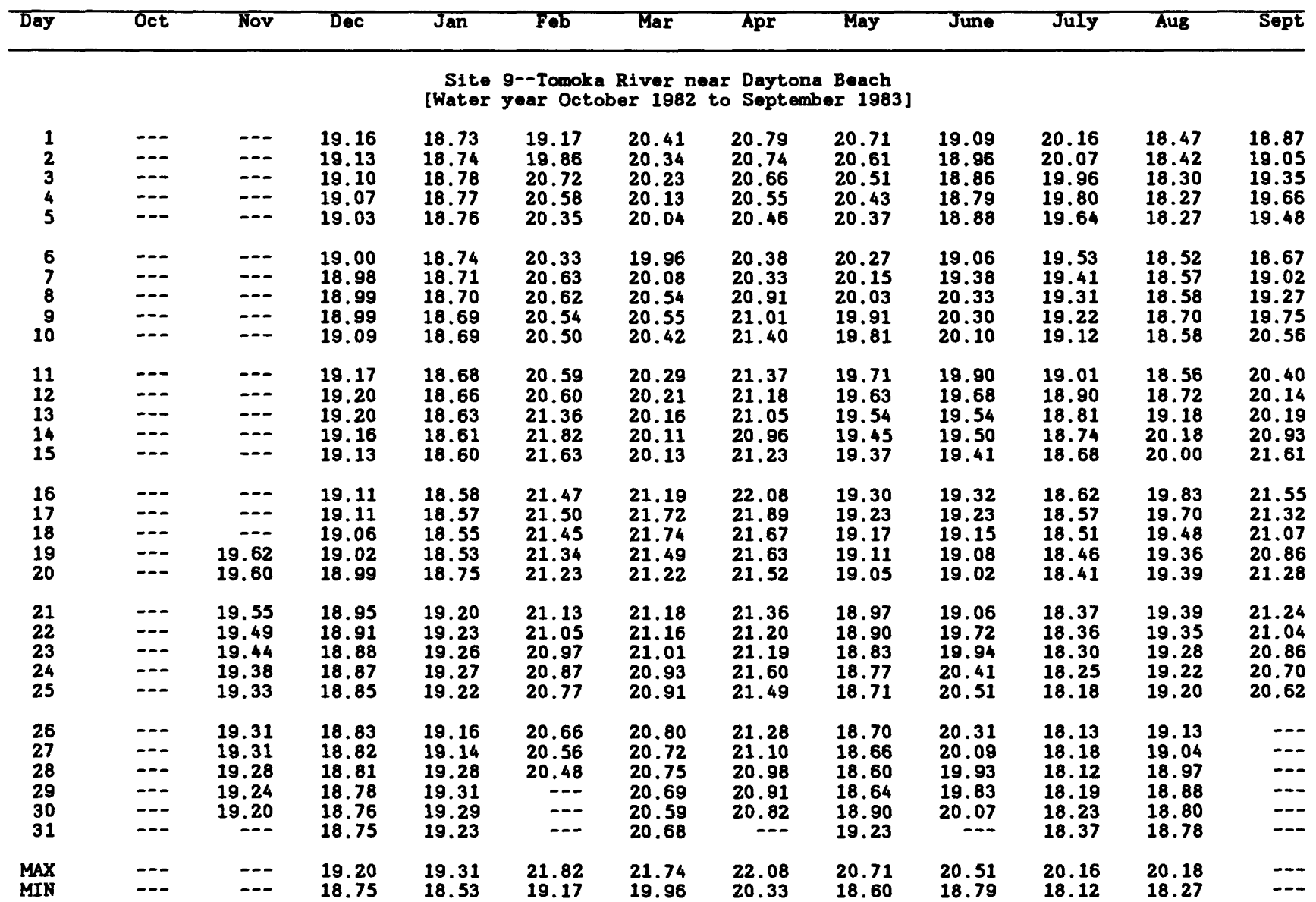

Site 9--Tomoka River near Daytona Beach [Water year October 1983 to September 1984]

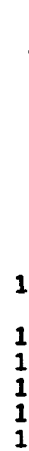

$20.23 \quad 19.65$

$2 \quad \ldots \quad 20.19 \quad 19.69$

$\begin{array}{llll}3 & \ldots & 20.19 & 19.69 \\ 4 & \ldots & 20.14 & 19.64\end{array}$

$\begin{array}{llll}4 & \ldots & 20.08 & 19.5 \\ 5 & \ldots & 20.02 & 19.48\end{array}$

$6 \quad \ldots \quad 19.97$

$7 \quad \ldots \quad 19.91$

$\begin{array}{lll}8 & \ldots & 19.86 \\ 9 & \ldots & 19.83\end{array}$

11
12

12
13
14
15

17

19
20

21

22

23

25

26
2
2
2
3
3

$27 \quad 20.50 \quad 19.68$

$20.52 \quad 19.52$

$29 \quad 20.43 \quad 19.53$

$30 \quad 20.35 \quad 19.63$

$\begin{array}{lll}\text { MAX } & -- & 20.23 \\ \text { MIN } & -- & 19.52\end{array}$
$21.62 \quad 20.22$

21.49

21.39

21.31

21.23

19.45

19.42

19.35

19.29
19.24

21. 14

21.07

21.01

20.94

20.90

19.25

21.56

21. 86

21.56

20.89

20.83

20.75

20.67

21.25

21.21

21.48

21.62
21.79

20.56

20.50

20.46

20.47

20.47

22.43

22.27

21.98

21.77

20.70

20.85

20.77

20.66

21.42

21.30

21.22

21.40

21.91

21.79

22.43

19.24

20.54

20.50

20.45

20.39

20.34

20.28

21.62

21.62
20.28
20.18
20.14

20.09

20.03

19.95

19.86

19.79

19.75
19.69

19.63

19.59

19.85

20.49

20.50

20.38

20.29

20.24

20.20
20.17

20.16

20.74

21.49

21.34

$20.70 \quad 19.10$

$20.57 \quad 20.12$

$20.51 \quad 21.16$

$20.45 \quad 21.00$

$20.39 \quad 20.84$

$20.31 \quad 20.70$

$\begin{array}{ll}20.22 & 20.60 \\ 20.16 & 20.57\end{array}$

20.10

20.00

19.93
19.85

19.74

19. 65

19.56

19.49

19.41

19.36

19.44

19.41

19.34

19.29

$20.96 \quad 19.22$

20.88

20.95

20.91

$$
\cdots
$$

19.23

19.31

19.24

$21.49 \quad 20.80$

$19.59 \quad 19.19$
20.52

20.45
20.37

20.31

20.46

20.47

20.38

20.27

20.17
20.11

20.07

20.03

19.99

19.96
19.87

19.77

19.68

19.58

19.49
19.40

19.31

19.24

19.16

19.11

19.24

19. 18

19.11

19.04

18.98
18.92

18.86

18.81

18.77

18.73

18.69

18.65

18.59

18.52

18.45

18.39

18.35

18.30

19.54

20.38

20.41

20.31

20.38

20.34

20.13

19.99

21.16

19.10
20.41

18.30
19.87

19.74

19.61

19.48

19. 23

19.13

19.04

18.96
18.89

18.83

18.78

18. 76

18.74

18.70

18.67

18.65

18.62

18.58
18.53

18.45

18.38

18.42

18.67

19.15

19.48

19.43

19.37

19.33

19.41

19.87

19.87
18.38
19.94

20.51

20.72

20.94

20.81

20.50

20.17

19.89

19.67

19.61

19.73

19.79

19.81

19.91

19.90

19.81

19.65
19.88

19.99

20.06

20.17

20.17

20.07

19.93

19.78

19.63

19.48

19.37

19.43

20.94 19.37

19.58

19.65

19.61

19.50

19.39

19.31

19.26

19.24

19.21
19.17

19.13

19.10

19.15

19.17

19.14

19.07

19.00

18.94

18.96

19.14

19.26

19.56

19.73

19.72

19.76

20.58

20.66

20.5

20.26

20.17 
APPENDIX II--Daily stage--Continued

[Mean values in feet above sea level]

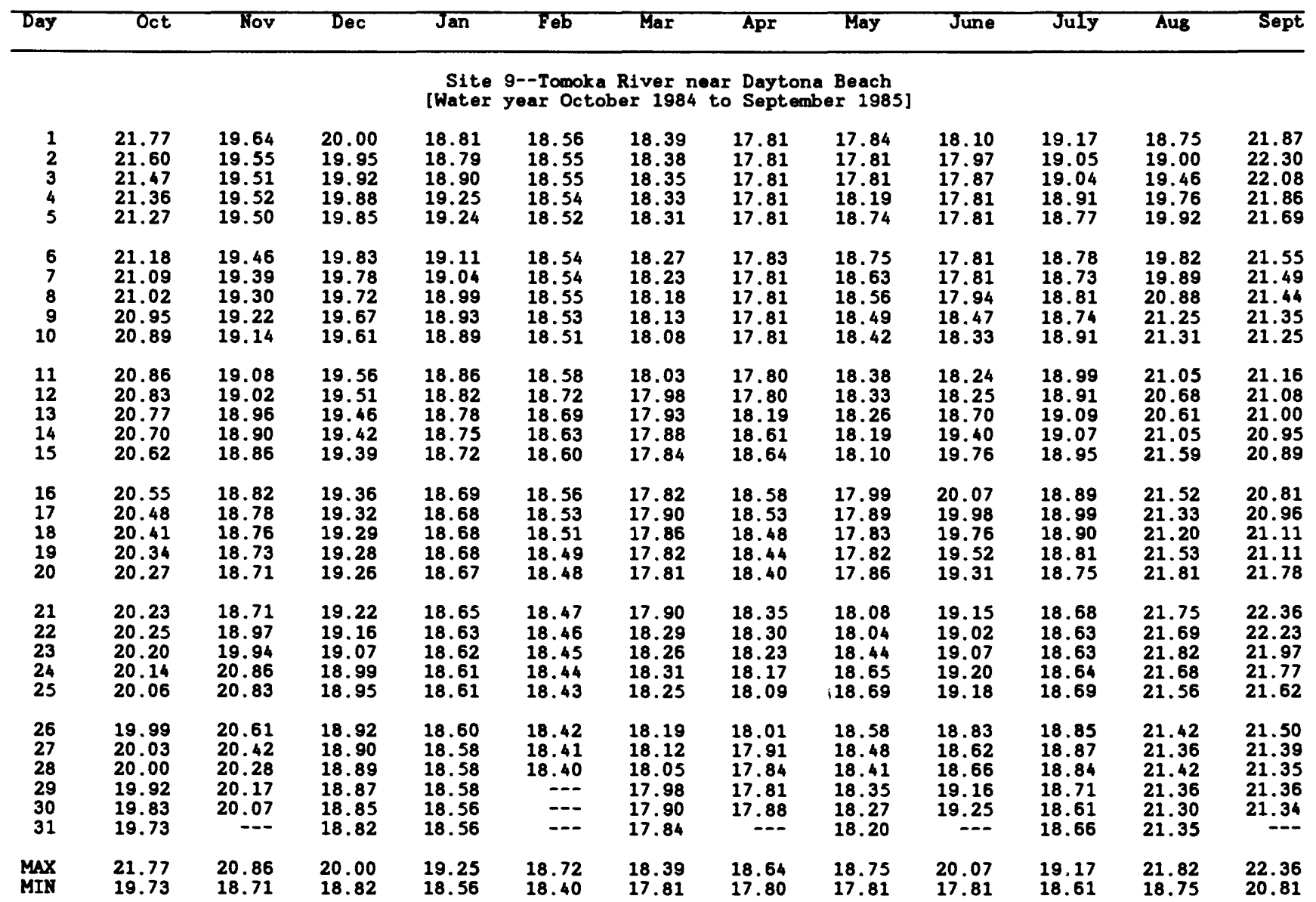

Site 10--B-19 Canal near Daytona Beach [Water year October 1982 to September 1983]

\begin{tabular}{|c|c|c|c|c|c|c|c|c|c|c|c|c|}
\hline $\begin{array}{l}1 \\
2 \\
3 \\
4 \\
5\end{array}$ & $\begin{array}{l}--- \\
--- \\
--- \\
--- \\
---\end{array}$ & $\begin{array}{l}--- \\
--- \\
--- \\
---\end{array}$ & $\begin{array}{l}--- \\
--- \\
---\end{array}$ & $\begin{array}{l}21.39 \\
21.39 \\
21.41 \\
21.38 \\
21.37\end{array}$ & $\begin{array}{l}21.41 \\
22.11 \\
22.38 \\
22.01 \\
21.78\end{array}$ & $\begin{array}{l}21.48 \\
21.43 \\
21.41 \\
21.40 \\
21.40\end{array}$ & $\begin{array}{l}21.95 \\
21.88 \\
21.81 \\
21.74 \\
21.69\end{array}$ & $\begin{array}{l}22.07 \\
22.03 \\
22.00 \\
22.02 \\
22.00\end{array}$ & $\begin{array}{l}21.23 \\
21.03 \\
20.93 \\
20.90 \\
21.01\end{array}$ & $\begin{array}{l}21.11 \\
21.10 \\
21.10 \\
21.09 \\
21.09\end{array}$ & $\begin{array}{l}21.32 \\
21.23 \\
21.20 \\
21.29 \\
21.31\end{array}$ & $\begin{array}{l}21.16 \\
21.66 \\
21.63 \\
21.39 \\
21.22\end{array}$ \\
\hline $\begin{array}{r}6 \\
7 \\
8 \\
9 \\
10\end{array}$ & $\begin{array}{l}--- \\
--- \\
--- \\
-- \\
---\end{array}$ & $\begin{array}{l}--- \\
--- \\
--- \\
---\end{array}$ & $\begin{array}{l}21.38 \\
21.57 \\
21.55 \\
21.49 \\
21.61\end{array}$ & $\begin{array}{l}21.36 \\
21.36 \\
21.36 \\
21.36 \\
21.37\end{array}$ & $\begin{array}{l}21.86 \\
22.04 \\
21.86 \\
21.72 \\
21.70\end{array}$ & $\begin{array}{l}21.39 \\
21.69 \\
21.95 \\
21.78 \\
21.63\end{array}$ & $\begin{array}{l}21.66 \\
21.69 \\
22.13 \\
22.23 \\
22.96\end{array}$ & $\begin{array}{l}21.96 \\
21.93 \\
21.91 \\
21.90 \\
21.89\end{array}$ & $\begin{array}{l}21.16 \\
21.41 \\
22.52 \\
22.31 \\
21.92\end{array}$ & $\begin{array}{l}21.09 \\
21.08 \\
21.09 \\
21.08 \\
21.07\end{array}$ & $\begin{array}{l}21.28 \\
21.31 \\
21.32 \\
21.50 \\
21.80\end{array}$ & $\begin{array}{l}21.13 \\
21.09 \\
21.04 \\
21.02 \\
21.01\end{array}$ \\
\hline $\begin{array}{l}11 \\
12 \\
13 \\
14 \\
15\end{array}$ & $\begin{array}{l}--- \\
--- \\
-- \\
--- \\
--\end{array}$ & $\begin{array}{l}-- \\
\cdots- \\
-\cdots \\
---\end{array}$ & $\begin{array}{l}21.63 \\
21.58 \\
21.53 \\
21.49 \\
21.48\end{array}$ & $\begin{array}{l}21.35 \\
21.34 \\
21.33 \\
21.33 \\
21.32\end{array}$ & $\begin{array}{l}21.77 \\
21.78 \\
23.14 \\
23.48 \\
22.87\end{array}$ & $\begin{array}{l}21.55 \\
21.51 \\
21.48 \\
21.47 \\
21.67\end{array}$ & $\begin{array}{l}22.86 \\
22.58 \\
22.32 \\
22.15 \\
22.75\end{array}$ & $\begin{array}{l}21.90 \\
21.93 \\
21.92 \\
21.91 \\
21.89\end{array}$ & $\begin{array}{l}21.61 \\
21.37 \\
21.28 \\
21.22 \\
21.14\end{array}$ & $\begin{array}{l}21.07 \\
21.07 \\
21.06 \\
21.07 \\
21.07\end{array}$ & $\begin{array}{l}21.54 \\
21.65 \\
21.63 \\
22.23 \\
22.28\end{array}$ & $\begin{array}{l}21.00 \\
20.99 \\
20.99 \\
21.00 \\
21.00\end{array}$ \\
\hline $\begin{array}{l}16 \\
17 \\
18 \\
19 \\
20\end{array}$ & $\begin{array}{l}--- \\
--- \\
-- \\
-- \\
--\end{array}$ & $\begin{array}{l}--- \\
--- \\
---\end{array}$ & $\begin{array}{l}21.50 \\
21.49 \\
21.46 \\
21.45 \\
21.44\end{array}$ & $\begin{array}{l}21.31 \\
21.31 \\
21.30 \\
21.29 \\
21.44\end{array}$ & $\begin{array}{l}22.50 \\
22.39 \\
22.17 \\
21.99 \\
21.83\end{array}$ & $\begin{array}{l}23.82 \\
24.30 \\
24.03 \\
23.26 \\
22.69\end{array}$ & $\begin{array}{l}24.16 \\
23.74 \\
23.21 \\
23.12 \\
22.78\end{array}$ & $\begin{array}{l}21.63 \\
21.24 \\
21.10 \\
20.98 \\
20.94\end{array}$ & $\begin{array}{l}21.05 \\
21.00 \\
20.98 \\
21.02 \\
21.03\end{array}$ & $\begin{array}{l}21.06 \\
21.06 \\
21.06 \\
21.08 \\
21.08\end{array}$ & $\begin{array}{l}21.62 \\
21.38 \\
21.24 \\
21.12 \\
21.06\end{array}$ & $\begin{array}{l}21.00 \\
21.10 \\
21.14 \\
21.13 \\
21.28\end{array}$ \\
\hline $\begin{array}{l}21 \\
22 \\
23 \\
24 \\
25\end{array}$ & $\begin{array}{l}--- \\
--- \\
--- \\
--- \\
---\end{array}$ & $\begin{array}{l}--- \\
--- \\
--- \\
--\end{array}$ & $\begin{array}{l}21.43 \\
21.41 \\
21.42 \\
21.42 \\
21.41\end{array}$ & $\begin{array}{l}21.52 \\
21.43 \\
21.43 \\
21.42 \\
21.40\end{array}$ & $\begin{array}{l}21.72 \\
21.66 \\
21.61 \\
21.56 \\
21.53\end{array}$ & $\begin{array}{l}22.74 \\
22.50 \\
22.20 \\
22.18 \\
22.03\end{array}$ & $\begin{array}{l}22.45 \\
22.21 \\
22.46 \\
23.49 \\
23.17\end{array}$ & $\begin{array}{l}20.91 \\
20.90 \\
20.89 \\
20.87 \\
20.86\end{array}$ & $\begin{array}{l}21.14 \\
21.56 \\
21.52 \\
21.63 \\
21.55\end{array}$ & $\begin{array}{l}21.08 \\
21.06 \\
21.09 \\
21.10 \\
21.09\end{array}$ & $\begin{array}{l}21.00 \\
20.96 \\
20.94 \\
20.92 \\
20.94\end{array}$ & $\begin{array}{l}21.22 \\
21.17 \\
21.12 \\
21.10 \\
21.51\end{array}$ \\
\hline $\begin{array}{l}26 \\
27 \\
28 \\
29 \\
30 \\
31\end{array}$ & $\begin{array}{l}--- \\
--- \\
-- \\
-- \\
-- \\
--\end{array}$ & $\begin{array}{l}-- \\
-- \\
--- \\
--- \\
--\end{array}$ & $\begin{array}{l}21.40 \\
21.40 \\
21.39 \\
21.38 \\
21.37 \\
21.38\end{array}$ & $\begin{array}{l}21.39 \\
21.45 \\
21.50 \\
21.44 \\
21.43 \\
21.42\end{array}$ & $\begin{array}{r}21.50 \\
21.48 \\
21.48 \\
.-- \\
.--\end{array}$ & $\begin{array}{l}21.85 \\
21.85 \\
21.93 \\
21.79 \\
21.70 \\
21.94\end{array}$ & $\begin{array}{l}22.75 \\
22.42 \\
22.21 \\
22.12 \\
22.09 \\
\ldots\end{array}$ & $\begin{array}{l}20.86 \\
20.83 \\
20.80 \\
21.02 \\
21.34 \\
21.42\end{array}$ & $\begin{array}{r}21.41 \\
21.29 \\
21.21 \\
21.15 \\
21.13 \\
-.-\end{array}$ & $\begin{array}{l}21.13 \\
21.15 \\
21.13 \\
21.17 \\
21.17 \\
21.34\end{array}$ & $\begin{array}{l}20.94 \\
20.92 \\
20.91 \\
20.93 \\
20.91 \\
20.89\end{array}$ & $\begin{array}{r}21.28 \\
21.20 \\
21.14 \\
21.11 \\
21.09 \\
---\end{array}$ \\
\hline $\begin{array}{l}\text { MAX } \\
\text { MIN }\end{array}$ & -- & -- & -- & $\begin{array}{l}21.52 \\
21.29\end{array}$ & $\begin{array}{l}23.48 \\
21.41\end{array}$ & $\begin{array}{l}24.30 \\
21.39\end{array}$ & $\begin{array}{l}24.16 \\
21.66\end{array}$ & $\begin{array}{l}22.07 \\
20.80\end{array}$ & $\begin{array}{l}22.52 \\
20.90\end{array}$ & $\begin{array}{l}21.34 \\
21.06\end{array}$ & $\begin{array}{l}22.28 \\
20.89\end{array}$ & $\begin{array}{l}21.66 \\
20.99\end{array}$ \\
\hline
\end{tabular}




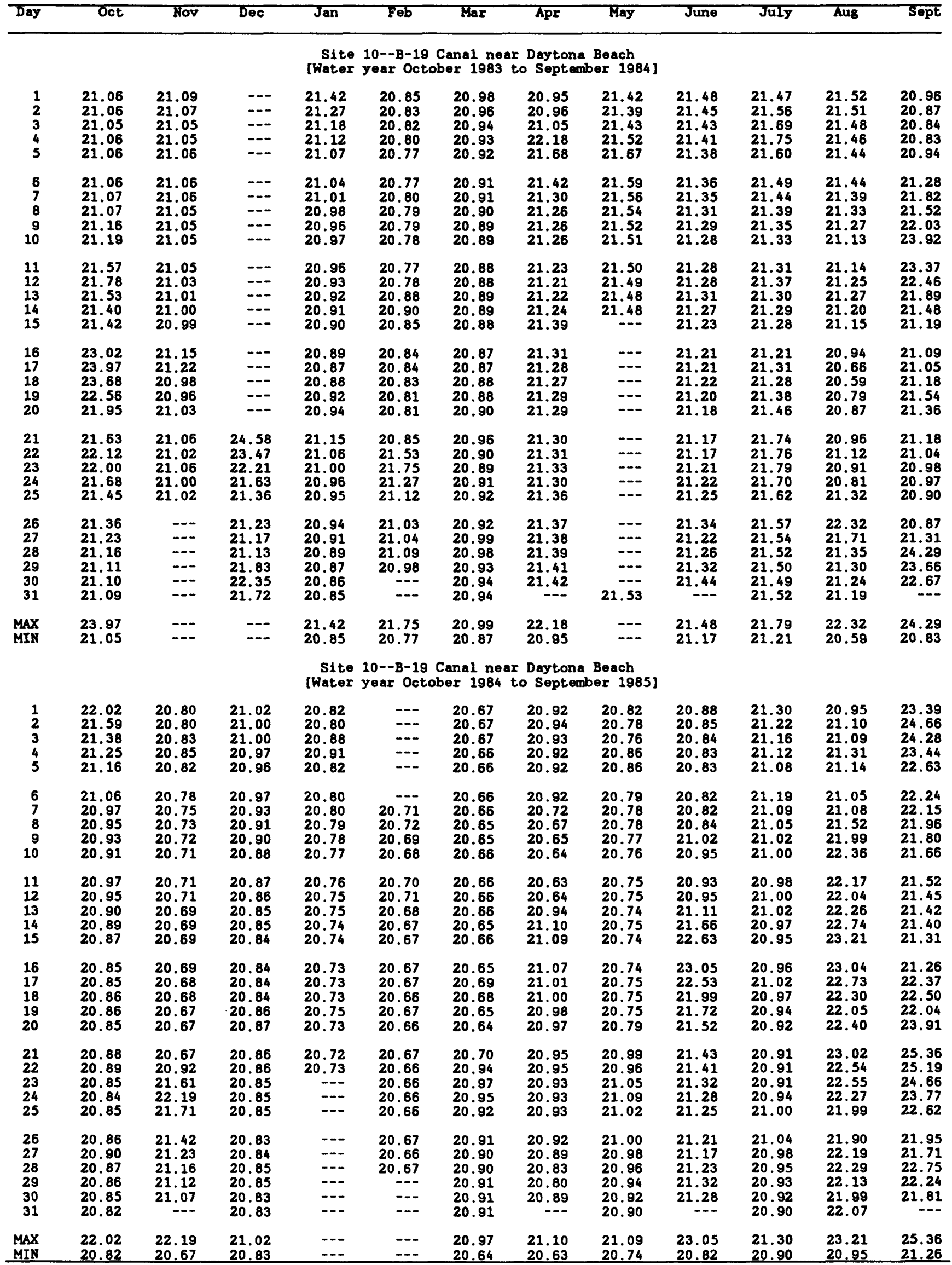


Appendix III 
APPEXDIX III--Daily discharge

[Mean values in cubic feet per second]

\begin{tabular}{|c|c|c|c|c|c|c|c|c|c|c|c|c|}
\hline Day & Oct & Nov & Dec & $\operatorname{Jan}$ & Feb & Mar & Apr & May & June & July & Aug & Sept \\
\hline & \multicolumn{12}{|c|}{$\begin{array}{l}\text { Site 1--Tomoka River near Bolly Bil1 } \\
\text { [Water year October } 1982 \text { to September 1983] }\end{array}$} \\
\hline $\begin{array}{l}1 \\
2 \\
3 \\
4 \\
5\end{array}$ & $\begin{array}{l}194 \\
405 \\
339 \\
272 \\
215\end{array}$ & $\begin{array}{l}78 \\
75 \\
78 \\
78 \\
73\end{array}$ & $\begin{array}{l}14 \\
13 \\
13 \\
12 \\
11\end{array}$ & $\begin{array}{l}8.9 \\
8.6 \\
9.3 \\
9.3 \\
8.9\end{array}$ & $\begin{array}{r}17 \\
59 \\
115 \\
112 \\
88\end{array}$ & $\begin{array}{l}80 \\
74 \\
69 \\
62 \\
56\end{array}$ & $\begin{array}{l}158 \\
139 \\
128 \\
113 \\
101\end{array}$ & $\begin{array}{r}106 \\
96 \\
88 \\
83 \\
79\end{array}$ & $\begin{array}{l}26 \\
16 \\
12 \\
11 \\
17\end{array}$ & $\begin{array}{l}50 \\
51 \\
46 \\
39 \\
34\end{array}$ & $\begin{array}{r}14 \\
7.2 \\
6.0 \\
4.6 \\
5.3\end{array}$ & $\begin{array}{l}14 \\
34 \\
40 \\
46 \\
39\end{array}$ \\
\hline $\begin{array}{r}6 \\
7 \\
8 \\
9 \\
10\end{array}$ & $\begin{array}{l}182 \\
153 \\
138 \\
120 \\
106\end{array}$ & $\begin{array}{l}67 \\
61 \\
58 \\
56 \\
52\end{array}$ & $\begin{array}{l}11 \\
11 \\
11 \\
12 \\
16\end{array}$ & $\begin{array}{l}8.4 \\
8.0 \\
7.8 \\
7.2 \\
6.8\end{array}$ & $\begin{array}{r}73 \\
100 \\
99 \\
82 \\
79\end{array}$ & $\begin{array}{r}53 \\
78 \\
127 \\
115 \\
95\end{array}$ & $\begin{array}{r}93 \\
88 \\
149 \\
161 \\
246\end{array}$ & $\begin{array}{l}70 \\
62 \\
56 \\
50 \\
45\end{array}$ & $\begin{array}{r}25 \\
35 \\
132 \\
110 \\
72\end{array}$ & $\begin{array}{l}30 \\
26 \\
25 \\
21 \\
17\end{array}$ & $\begin{array}{r}4.4 \\
5.5 \\
8.2 \\
9.8 \\
54\end{array}$ & $\begin{array}{l}33 \\
27 \\
23 \\
20 \\
17\end{array}$ \\
\hline $\begin{array}{l}11 \\
12 \\
13 \\
14 \\
15\end{array}$ & $\begin{array}{r}101 \\
104 \\
97 \\
92 \\
97\end{array}$ & $\begin{array}{l}49 \\
46 \\
43 \\
40 \\
37\end{array}$ & $\begin{array}{l}20 \\
16 \\
15 \\
14 \\
13\end{array}$ & $\begin{array}{l}6.5 \\
6.1 \\
5.8 \\
5.5 \\
5.2\end{array}$ & $\begin{array}{r}85 \\
85 \\
227 \\
323 \\
242\end{array}$ & $\begin{array}{l}83 \\
75 \\
69 \\
65 \\
70\end{array}$ & $\begin{array}{l}237 \\
195 \\
165 \\
147 \\
151\end{array}$ & $\begin{array}{l}43 \\
39 \\
35 \\
31 \\
28\end{array}$ & $\begin{array}{l}60 \\
58 \\
65 \\
57 \\
45\end{array}$ & $\begin{array}{r}14 \\
11 \\
8.9 \\
7.6 \\
6.5\end{array}$ & $\begin{array}{l}27 \\
19 \\
31 \\
59 \\
83\end{array}$ & $\begin{array}{r}14 \\
11 \\
9.8 \\
9.1 \\
8.2\end{array}$ \\
\hline $\begin{array}{l}16 \\
17 \\
18 \\
19 \\
20\end{array}$ & $\begin{array}{l}88 \\
81 \\
76 \\
72 \\
70\end{array}$ & $\begin{array}{l}35 \\
33 \\
31 \\
31 \\
29\end{array}$ & $\begin{array}{l}13 \\
14 \\
13 \\
12 \\
12\end{array}$ & $\begin{array}{l}4.9 \\
4.6 \\
4.4 \\
4.1 \\
7.2\end{array}$ & $\begin{array}{l}201 \\
218 \\
205 \\
176 \\
158\end{array}$ & $\begin{array}{l}213 \\
332 \\
325 \\
250 \\
196\end{array}$ & $\begin{array}{l}369 \\
320 \\
260 \\
262 \\
237\end{array}$ & $\begin{array}{l}25 \\
22 \\
20 \\
17 \\
16\end{array}$ & $\begin{array}{l}39 \\
36 \\
31 \\
27 \\
23\end{array}$ & $\begin{array}{l}5.6 \\
4.9 \\
4.4 \\
4.0 \\
3.6\end{array}$ & $\begin{array}{l}79 \\
61 \\
52 \\
46 \\
39\end{array}$ & $\begin{array}{l}7.2 \\
10 \\
30 \\
31 \\
38\end{array}$ \\
\hline $\begin{array}{l}21 \\
22 \\
23 \\
24 \\
25\end{array}$ & $\begin{array}{r}67 \\
64 \\
71 \\
150 \\
169\end{array}$ & $\begin{array}{l}27 \\
24 \\
22 \\
21 \\
19\end{array}$ & $\begin{array}{l}11 \\
11 \\
11 \\
10 \\
10\end{array}$ & $\begin{array}{l}20 \\
16 \\
13 \\
13 \\
13\end{array}$ & $\begin{array}{l}145 \\
134 \\
124 \\
115 \\
105\end{array}$ & $\begin{array}{l}187 \\
173 \\
154 \\
149 \\
141\end{array}$ & $\begin{array}{l}205 \\
176 \\
167 \\
233 \\
226\end{array}$ & $\begin{array}{r}13 \\
12 \\
10 \\
8.9 \\
8.0\end{array}$ & $\begin{array}{l}24 \\
56 \\
57 \\
78 \\
86\end{array}$ & $\begin{array}{l}3.4 \\
3.3 \\
3.2 \\
3.1 \\
2.9\end{array}$ & $\begin{array}{l}35 \\
34 \\
32 \\
30 \\
27\end{array}$ & $\begin{array}{l}40 \\
37 \\
31 \\
25 \\
46\end{array}$ \\
\hline $\begin{array}{l}26 \\
27 \\
28 \\
29 \\
30 \\
31\end{array}$ & $\begin{array}{r}146 \\
122 \\
106 \\
96 \\
89 \\
83\end{array}$ & $\begin{array}{l}20 \\
20 \\
19 \\
17 \\
16 \\
--\end{array}$ & $\begin{array}{l}9.8 \\
9.6 \\
9.3 \\
9.1 \\
8.9 \\
8.9\end{array}$ & $\begin{array}{l}13 \\
14 \\
18 \\
19 \\
18 \\
17\end{array}$ & $\begin{array}{r}95 \\
88 \\
82 \\
--- \\
-- \\
---\end{array}$ & $\begin{array}{l}128 \\
121 \\
129 \\
119 \\
109 \\
145\end{array}$ & $\begin{array}{l}190 \\
160 \\
139 \\
126 \\
117 \\
---\end{array}$ & $\begin{array}{c}7.6 \\
6.3 \\
5.6 \\
7.2 \\
28 \\
45\end{array}$ & $\begin{array}{r}77 \\
62 \\
50 \\
43 \\
43 \\
---\end{array}$ & $\begin{array}{l}2.9 \\
4.1 \\
3.7 \\
4.5 \\
6.3 \\
7.8\end{array}$ & $\begin{array}{l}28 \\
27 \\
22 \\
19 \\
16 \\
12\end{array}$ & $\begin{array}{r}54 \\
45 \\
35 \\
28 \\
25 \\
---\end{array}$ \\
\hline
\end{tabular}

Site 1--Tomoka River near Bolly Hill

[Water year October 1983 to September 1984]

\begin{tabular}{|c|c|c|c|c|c|c|c|c|c|c|c|c|}
\hline $\begin{array}{l}1 \\
2 \\
3 \\
4 \\
5\end{array}$ & $\begin{array}{l}28 \\
22 \\
18 \\
16 \\
14\end{array}$ & $\begin{array}{l}78 \\
74 \\
69 \\
65 \\
62\end{array}$ & $\begin{array}{l}45 \\
41 \\
40 \\
38 \\
37\end{array}$ & $\begin{array}{l}300 \\
260 \\
220 \\
200 \\
180\end{array}$ & $\begin{array}{l}66 \\
62 \\
59 \\
57 \\
53\end{array}$ & $\begin{array}{r}130 \\
116 \\
106 \\
97 \\
90\end{array}$ & $\begin{array}{r}19 \\
18 \\
22 \\
303 \\
332\end{array}$ & $\begin{array}{l}24 \\
22 \\
20 \\
20 \\
42\end{array}$ & $\begin{array}{l}48 \\
42 \\
36 \\
31 \\
27\end{array}$ & $\begin{array}{r}42 \\
138 \\
167 \\
208 \\
165\end{array}$ & $\begin{array}{l}34 \\
36 \\
35 \\
35 \\
32\end{array}$ & $\begin{array}{l}56 \\
46 \\
37 \\
32 \\
48\end{array}$ \\
\hline $\begin{array}{r}6 \\
7 \\
8 \\
9 \\
10\end{array}$ & $\begin{array}{l}12 \\
11 \\
12 \\
27 \\
22\end{array}$ & $\begin{array}{l}61 \\
57 \\
56 \\
53 \\
54\end{array}$ & $\begin{array}{l}35 \\
32 \\
30 \\
28 \\
27\end{array}$ & $\begin{array}{l}160 \\
150 \\
140 \\
135 \\
145\end{array}$ & $\begin{array}{l}49 \\
45 \\
41 \\
39 \\
36\end{array}$ & $\begin{array}{l}83 \\
77 \\
69 \\
63 \\
58\end{array}$ & $\begin{array}{l}228 \\
171 \\
142 \\
123 \\
120\end{array}$ & $\begin{array}{l}28 \\
22 \\
18 \\
16 \\
14\end{array}$ & $\begin{array}{l}23 \\
20 \\
18 \\
15 \\
13\end{array}$ & $\begin{array}{r}123 \\
93 \\
73 \\
58 \\
50\end{array}$ & $\begin{array}{l}30 \\
29 \\
28 \\
26 \\
24\end{array}$ & $\begin{array}{l}138 \\
322 \\
301 \\
343 \\
735\end{array}$ \\
\hline $\begin{array}{l}11 \\
12 \\
13 \\
14 \\
15\end{array}$ & $\begin{array}{l}23 \\
43 \\
59 \\
52 \\
43\end{array}$ & $\begin{array}{l}53 \\
52 \\
51 \\
49 \\
49\end{array}$ & $\begin{array}{r}27 \\
377 \\
375 \\
270 \\
235\end{array}$ & $\begin{array}{l}152 \\
146 \\
139 \\
124 \\
110\end{array}$ & $\begin{array}{r}34 \\
33 \\
49 \\
118 \\
106\end{array}$ & $\begin{array}{l}55 \\
51 \\
49 \\
45 \\
40\end{array}$ & $\begin{array}{r}108 \\
96 \\
86 \\
80 \\
120\end{array}$ & $\begin{array}{r}12 \\
10 \\
9.1 \\
8.4 \\
7.9\end{array}$ & $\begin{array}{c}13 \\
12 \\
12 \\
12 \\
9.3\end{array}$ & $\begin{array}{l}47 \\
47 \\
48 \\
52 \\
51\end{array}$ & $\begin{array}{l}22 \\
21 \\
25 \\
22 \\
19\end{array}$ & $\begin{array}{l}556 \\
410 \\
324 \\
270 \\
224\end{array}$ \\
\hline $\begin{array}{l}16 \\
17 \\
18 \\
19 \\
20\end{array}$ & $\begin{array}{l}175 \\
282 \\
317 \\
230 \\
182\end{array}$ & $\begin{array}{l}52 \\
51 \\
49 \\
46 \\
46\end{array}$ & $\begin{array}{l}216 \\
201 \\
239 \\
278 \\
368\end{array}$ & $\begin{array}{r}103 \\
96 \\
90 \\
100 \\
99\end{array}$ & $\begin{array}{l}90 \\
78 \\
69 \\
64 \\
61\end{array}$ & $\begin{array}{l}36 \\
33 \\
31 \\
28 \\
27\end{array}$ & $\begin{array}{r}107 \\
88 \\
75 \\
65 \\
59\end{array}$ & $\begin{array}{l}7.4 \\
6.9 \\
6.3 \\
5.9 \\
5.6\end{array}$ & $\begin{array}{r}8.2 \\
7.8 \\
8.9 \\
10 \\
8.5\end{array}$ & $\begin{array}{l}50 \\
46 \\
41 \\
39 \\
49\end{array}$ & $\begin{array}{l}18 \\
18 \\
19 \\
18 \\
23\end{array}$ & $\begin{array}{l}183 \\
161 \\
160 \\
207 \\
216\end{array}$ \\
\hline $\begin{array}{l}21 \\
22 \\
23 \\
24 \\
25\end{array}$ & $\begin{array}{l}148 \\
181 \\
235 \\
212 \\
173\end{array}$ & $\begin{array}{l}55 \\
52 \\
49 \\
47 \\
50\end{array}$ & $\begin{array}{l}816 \\
648 \\
520 \\
400 \\
340\end{array}$ & $\begin{array}{l}122 \\
142 \\
134 \\
122 \\
112\end{array}$ & $\begin{array}{r}61 \\
109 \\
266 \\
237 \\
197\end{array}$ & $\begin{array}{l}30 \\
29 \\
27 \\
25 \\
23\end{array}$ & $\begin{array}{l}55 \\
51 \\
49 \\
48 \\
44\end{array}$ & $\begin{array}{r}5.4 \\
5.5 \\
96 \\
155 \\
94\end{array}$ & $\begin{array}{c}7.8 \\
7.3 \\
8.4 \\
13 \\
11\end{array}$ & $\begin{array}{r}70 \\
99 \\
101 \\
91 \\
70\end{array}$ & $\begin{array}{l}28 \\
36 \\
33 \\
35 \\
51\end{array}$ & $\begin{array}{l}195 \\
169 \\
149 \\
134 \\
120\end{array}$ \\
\hline $\begin{array}{l}26 \\
27 \\
28 \\
29 \\
30 \\
31\end{array}$ & $\begin{array}{r}151 \\
132 \\
109 \\
97 \\
88 \\
82\end{array}$ & $\begin{array}{r}58 \\
48 \\
42 \\
44 \\
49 \\
---\end{array}$ & $\begin{array}{l}270 \\
220 \\
190 \\
300 \\
400 \\
350\end{array}$ & $\begin{array}{r}105 \\
98 \\
91 \\
83 \\
77 \\
72\end{array}$ & $\begin{array}{l}165 \\
143 \\
157 \\
146 \\
--- \\
---\end{array}$ & $\begin{array}{l}22 \\
23 \\
25 \\
23 \\
22 \\
20\end{array}$ & $\begin{array}{r}39 \\
35 \\
32 \\
28 \\
26 \\
--0\end{array}$ & $\begin{array}{r}82 \\
108 \\
106 \\
80 \\
63 \\
55\end{array}$ & $\begin{array}{l}22 \\
25 \\
24 \\
25 \\
29 \\
---\end{array}$ & $\begin{array}{l}56 \\
50 \\
44 \\
37 \\
32 \\
33\end{array}$ & $\begin{array}{l}87 \\
97 \\
87 \\
73 \\
62 \\
59\end{array}$ & $\begin{array}{l}107 \\
114 \\
649 \\
579 \\
446 \\
---\end{array}$ \\
\hline
\end{tabular}


APPENDIX III--Daily discharge--Continued

[Mean values in cubic feet per second]

\begin{tabular}{|c|c|c|c|c|c|c|c|c|c|c|c|c|}
\hline Day & Oct & Tov & Dec & $\operatorname{Jan}$ & Feb & Mar & $\overline{\text { Apr }}$ & May & June & July & Alus & Sept \\
\hline \multicolumn{13}{|c|}{$\begin{array}{l}\text { Site 1--Tomoka River near Bolly Bill } \\
\text { [Water year October } 1984 \text { to September 1985] }\end{array}$} \\
\hline $\begin{array}{l}1 \\
2 \\
3 \\
4 \\
5\end{array}$ & $\begin{array}{l}356 \\
290 \\
243 \\
213 \\
189\end{array}$ & $\begin{array}{l}26 \\
24 \\
24 \\
24 \\
23\end{array}$ & $\begin{array}{l}42 \\
39 \\
37 \\
36 \\
37\end{array}$ & $\begin{array}{l}8.5 \\
8.2 \\
11 \\
25 \\
19\end{array}$ & $\begin{array}{l}4.6 \\
4.5 \\
4.5 \\
4.3 \\
4.3\end{array}$ & $\begin{array}{l}2.7 \\
2.6 \\
2.5 \\
2.5 \\
2.4\end{array}$ & $\begin{array}{l}1.6 \\
1.8 \\
1.7 \\
1.6 \\
1.5\end{array}$ & $\begin{array}{r}2.9 \\
2.0 \\
1.7 \\
5.1 \\
21\end{array}$ & $\begin{array}{l}1.5 \\
1.4 \\
1.3 \\
1.2 \\
1.1\end{array}$ & $\begin{array}{l}11 \\
9.1 \\
6.9 \\
5.8 \\
5.0\end{array}$ & $\begin{array}{l}14 \\
33 \\
24 \\
35 \\
30\end{array}$ & $\begin{array}{l}450 \\
712 \\
449 \\
335 \\
283\end{array}$ \\
\hline $\begin{array}{r}6 \\
7 \\
8 \\
9 \\
10\end{array}$ & $\begin{array}{l}169 \\
153 \\
141 \\
135 \\
136\end{array}$ & $\begin{array}{l}21 \\
19 \\
17 \\
16 \\
15\end{array}$ & $\begin{array}{l}35 \\
32 \\
30 \\
27 \\
25\end{array}$ & $\begin{array}{c}16 \\
13 \\
11 \\
9.6 \\
9.1\end{array}$ & $\begin{array}{l}5.0 \\
5.6 \\
5.6 \\
5.1 \\
4.5\end{array}$ & $\begin{array}{l}2.3 \\
2.2 \\
2.1 \\
2.0 \\
2.0\end{array}$ & $\begin{array}{l}1.7 \\
3.1 \\
2.5 \\
1.9 \\
1.7\end{array}$ & $\begin{array}{r}10 \\
5.9 \\
3.9 \\
3.0 \\
2.6\end{array}$ & $\begin{array}{l}1.0 \\
1.0 \\
1.2 \\
2.0 \\
3.6\end{array}$ & $\begin{array}{c}8.9 \\
8.6 \\
17 \\
10 \\
6.2\end{array}$ & $\begin{array}{r}27 \\
26 \\
119 \\
188 \\
175\end{array}$ & $\begin{array}{l}255 \\
242 \\
218 \\
189 \\
170\end{array}$ \\
\hline $\begin{array}{l}11 \\
12 \\
13 \\
14 \\
15\end{array}$ & $\begin{array}{r}133 \\
127 \\
111 \\
92 \\
80\end{array}$ & $\begin{array}{c}14 \\
13 \\
12 \\
11 \\
9.9\end{array}$ & $\begin{array}{l}24 \\
23 \\
21 \\
20 \\
19\end{array}$ & $\begin{array}{l}8.4 \\
7.8 \\
7.2 \\
6.9 \\
6.6\end{array}$ & $\begin{array}{l}4.5 \\
5.0 \\
4.6 \\
4.2 \\
4.1\end{array}$ & $\begin{array}{l}2.0 \\
1.9 \\
1.8 \\
1.8 \\
1.8\end{array}$ & $\begin{array}{r}1.6 \\
1.6 \\
6.0 \\
14.9 \\
7.9\end{array}$ & $\begin{array}{l}2.4 \\
2.3 \\
2.0 \\
2.2 \\
2.0\end{array}$ & $\begin{array}{l}2.4 \\
1.8 \\
6.7 \\
20 \\
44\end{array}$ & $\begin{array}{l}5.1 \\
6.1 \\
9.2 \\
8.0 \\
6.5\end{array}$ & $\begin{array}{l}173 \\
165 \\
117 \\
113 \\
179\end{array}$ & $\begin{array}{l}155 \\
141 \\
130 \\
127 \\
117\end{array}$ \\
\hline $\begin{array}{l}16 \\
17 \\
18 \\
19 \\
20\end{array}$ & $\begin{array}{l}72 \\
66 \\
60 \\
55 \\
51\end{array}$ & $\begin{array}{l}9.3 \\
8.8 \\
8.2 \\
7.8 \\
7.4\end{array}$ & $\begin{array}{l}18 \\
17 \\
16 \\
16 \\
15\end{array}$ & $\begin{array}{l}6.2 \\
6.1 \\
6.1 \\
6.4 \\
6.1\end{array}$ & $\begin{array}{l}3.9 \\
3.8 \\
3.6 \\
3.7 \\
3.5\end{array}$ & $\begin{array}{l}1.7 \\
2.5 \\
2.9 \\
2.2 \\
1.8\end{array}$ & $\begin{array}{l}5.4 \\
3.7 \\
2.9 \\
2.6 \\
2.3\end{array}$ & $\begin{array}{l}1.9 \\
1.9 \\
2.0 \\
1.9 \\
3.0\end{array}$ & $\begin{array}{l}83 \\
48 \\
30 \\
20 \\
15\end{array}$ & $\begin{array}{l}7.3 \\
19 \\
16 \\
13 \\
11\end{array}$ & $\begin{array}{l}201 \\
179 \\
154 \\
184 \\
269\end{array}$ & $\begin{array}{l}106 \\
125 \\
168 \\
158 \\
280\end{array}$ \\
\hline $\begin{array}{l}21 \\
22 \\
23 \\
24 \\
25\end{array}$ & $\begin{array}{l}50 \\
53 \\
47 \\
44 \\
39\end{array}$ & $\begin{array}{c}7.2 \\
14 \\
57 \\
114 \\
103\end{array}$ & $\begin{array}{l}14 \\
14 \\
13 \\
12 \\
12\end{array}$ & $\begin{array}{l}5.6 \\
5.4 \\
5.4 \\
5.5 \\
5.8\end{array}$ & $\begin{array}{l}3.4 \\
3.4 \\
3.3 \\
3.2 \\
3.0\end{array}$ & $\begin{array}{c}2.5 \\
11 \\
7.1 \\
3.8 \\
2.8\end{array}$ & $\begin{array}{l}2.1 \\
2.2 \\
2.1 \\
2.0 \\
1.9\end{array}$ & $\begin{array}{l}9.2 \\
5.4 \\
8.0 \\
23 \\
12\end{array}$ & $\begin{array}{c}12 \\
9.4 \\
8.5 \\
14 \\
19\end{array}$ & $\begin{array}{c}9.3 \\
8.1 \\
7.2 \\
16 \\
47\end{array}$ & $\begin{array}{l}270 \\
243 \\
293 \\
248 \\
214\end{array}$ & $\begin{array}{l}700 \\
611 \\
416 \\
316 \\
269\end{array}$ \\
\hline $\begin{array}{l}26 \\
27 \\
28 \\
29 \\
30 \\
31\end{array}$ & $\begin{array}{l}36 \\
37 \\
36 \\
33 \\
30 \\
28\end{array}$ & $\begin{array}{l}85 \\
70 \\
59 \\
52 \\
47 \\
---\end{array}$ & $\begin{array}{r}11 \\
11 \\
11 \\
10 \\
9.5 \\
8.9\end{array}$ & $\begin{array}{l}5.2 \\
4.9 \\
4.9 \\
4.8 \\
4.6 \\
4.7\end{array}$ & $\begin{array}{l}2.9 \\
2.8 \\
2.8 \\
--- \\
--- \\
---\end{array}$ & $\begin{array}{l}2.3 \\
2.0 \\
1.9 \\
1.8 \\
1.7 \\
1.6\end{array}$ & $\begin{array}{l}1.8 \\
1.8 \\
1.7 \\
1.6 \\
2.1 \\
-.-\end{array}$ & $\begin{array}{l}5.8 \\
3.7 \\
2.7 \\
2.1 \\
1.9 \\
1.7\end{array}$ & $\begin{array}{c}19 \\
11 \\
7.9 \\
11 \\
11 \\
-\end{array}$ & $\begin{array}{c}30 \\
22 \\
18 \\
14 \\
12 \\
9.7\end{array}$ & $\begin{array}{l}188 \\
176 \\
199 \\
185 \\
169 \\
181\end{array}$ & $\begin{array}{l}234 \\
201 \\
206 \\
194 \\
187 \\
---\end{array}$ \\
\hline
\end{tabular}

Site 2--Eleventh Street Canal near Holly Bill

[Water year October 1982 to September 1983]

\begin{tabular}{|c|c|c|c|c|c|c|c|c|c|c|}
\hline $\begin{array}{l}-- \\
-- \\
\cdots- \\
--\end{array}$ & $\begin{array}{l}--- \\
--- \\
--- \\
---\end{array}$ & $\begin{array}{l}--- \\
--. \\
2.8 \\
2.7 \\
2.7\end{array}$ & $\begin{array}{l}2.5 \\
2.5 \\
2.4 \\
2.4 \\
2.4\end{array}$ & $\begin{array}{r}2.7 \\
12 \\
7.6 \\
5.9 \\
5.2\end{array}$ & $\begin{array}{l}4.5 \\
4.3 \\
4.1 \\
3.9 \\
3.9\end{array}$ & $\begin{array}{c}10 \\
8.8 \\
8.0 \\
6.9 \\
6.2\end{array}$ & $\begin{array}{l}3.9 \\
3.7 \\
3.6 \\
3.8 \\
---\end{array}$ & $\begin{array}{l}4.5 \\
3.9 \\
3.7 \\
3.6 \\
5.2\end{array}$ & $\begin{array}{l}3.3 \\
3.2 \\
3.1 \\
3.0 \\
2.9\end{array}$ & $\begin{array}{r}0.76 \\
.34 \\
.32 \\
.24 \\
.25\end{array}$ \\
\hline $\begin{array}{l}--- \\
\cdots- \\
\cdots-\end{array}$ & $\begin{array}{l}--- \\
-- \\
--- \\
---\end{array}$ & $\begin{array}{l}2.7 \\
2.9 \\
2.8 \\
3.0 \\
3.2\end{array}$ & $\begin{array}{l}2.4 \\
2.4 \\
2.4 \\
2.3 \\
2.3\end{array}$ & $\begin{array}{l}6.4 \\
6.4 \\
5.5 \\
5.0 \\
5.3\end{array}$ & $\begin{array}{l}3.7 \\
8.5 \\
8.8 \\
7.2 \\
6.3\end{array}$ & $\begin{array}{l}5.6 \\
6.3 \\
12 \\
11 \\
22\end{array}$ & $\begin{array}{l}--- \\
--- \\
-- \\
3.4 \\
3.3\end{array}$ & $\begin{array}{c}5.5 \\
6.7 \\
10 \\
7.7 \\
6.2\end{array}$ & $\begin{array}{l}2.9 \\
2.8 \\
2.9 \\
2.8 \\
2.7\end{array}$ & $\begin{array}{l}.18 \\
.29 \\
2.3 \\
3.2 \\
4.2\end{array}$ \\
\hline $\begin{array}{l}--- \\
-\cdots \\
--- \\
--\end{array}$ & $\begin{array}{l}--- \\
--- \\
--- \\
---\end{array}$ & $\begin{array}{l}3.1 \\
3.0 \\
3.1 \\
3.0 \\
2.9\end{array}$ & $\begin{array}{l}2.3 \\
2.3 \\
2.3 \\
2.3 \\
2.3\end{array}$ & $\begin{array}{l}5.4 \\
5.3 \\
26 \\
21 \\
13\end{array}$ & $\begin{array}{l}5.6 \\
5.1 \\
4.7 \\
4.5 \\
5.8\end{array}$ & $\begin{array}{c}15 \\
11 \\
9.2 \\
7.9 \\
8.1\end{array}$ & $\begin{array}{l}3.2 \\
3.2 \\
3.2 \\
3.1 \\
3.0\end{array}$ & $\begin{array}{l}5.4 \\
5.0 \\
4.6 \\
4.3 \\
3.9\end{array}$ & $\begin{array}{l}2.6 \\
2.5 \\
2.6 \\
1.2 \\
.37\end{array}$ & $\begin{array}{l}3.8 \\
3.2 \\
3.9 \\
4.2 \\
4.1\end{array}$ \\
\hline $\begin{array}{l}--- \\
-- \\
--- \\
--- \\
--\end{array}$ & $\begin{array}{l}-- \\
-- \\
-- \\
--\end{array}$ & $\begin{array}{l}2.8 \\
2.8 \\
2.7 \\
2.7 \\
2.7\end{array}$ & $\begin{array}{l}2.2 \\
4.1 \\
3.2 \\
2.8 \\
2.9\end{array}$ & $\begin{array}{c}12 \\
13 \\
11 \\
9.1 \\
8.1\end{array}$ & $\begin{array}{l}20 \\
35 \\
25 \\
16 \\
13\end{array}$ & $\begin{array}{l}9.1 \\
7.4 \\
6.8 \\
9.2 \\
7.4\end{array}$ & $\begin{array}{l}3.0 \\
3.0 \\
2.9 \\
2.9 \\
2.9\end{array}$ & $\begin{array}{l}3.7 \\
3.7 \\
3.5 \\
3.4 \\
3.3\end{array}$ & $\begin{array}{l}.32 \\
.25 \\
.21 \\
.20 \\
.19\end{array}$ & $\begin{array}{l}3.7 \\
3.6 \\
3.4 \\
3.2 \\
3.1\end{array}$ \\
\hline $\begin{array}{l}m- \\
\cdots- \\
-- \\
-- \\
\end{array}$ & $\begin{array}{l}-- \\
-- \\
-- \\
--\end{array}$ & $\begin{array}{l}2.6 \\
2.6 \\
2.5 \\
2.6 \\
2.5\end{array}$ & $\begin{array}{l}2.8 \\
2.7 \\
2.7 \\
3.4 \\
3.1\end{array}$ & $\begin{array}{l}7.3 \\
6.7 \\
6.1 \\
5.6 \\
5.1\end{array}$ & $\begin{array}{c}13 \\
10 \\
8.7 \\
9.8 \\
8.5\end{array}$ & $\begin{array}{l}6.4 \\
5.7 \\
6.8 \\
8.4 \\
6.6\end{array}$ & $\begin{array}{l}2.8 \\
2.7 \\
2.7 \\
2.6 \\
2.6\end{array}$ & $\begin{array}{l}4.1 \\
7.5 \\
5.9 \\
6.3 \\
5.2\end{array}$ & $\begin{array}{l}.15 \\
.11 \\
.10 \\
.09 \\
.07\end{array}$ & $\begin{array}{l}2.8 \\
2.6 \\
2.7 \\
2.8 \\
2.8\end{array}$ \\
\hline $\begin{array}{l}\cdots \\
\cdots \\
\cdots \\
\cdots\end{array}$ & $\begin{array}{l}--- \\
--- \\
--- \\
--- \\
--\end{array}$ & $\begin{array}{l}2.5 \\
2.5 \\
2.5 \\
2.5 \\
2.6 \\
2.6\end{array}$ & $\begin{array}{l}--- \\
--. \\
2.9 \\
2.9 \\
2.8 \\
2.8\end{array}$ & $\begin{array}{l}4.7 \\
4.5 \\
4.5 \\
-.- \\
---\end{array}$ & $\begin{array}{r}7.4 \\
7.3 \\
7.4 \\
6.4 \\
5.8 \\
11\end{array}$ & $\begin{array}{l}5.5 \\
4.8 \\
4.5 \\
4.2 \\
4.1 \\
-.-\end{array}$ & $\begin{array}{l}2.7 \\
2.6 \\
2.5 \\
3.3 \\
6.0 \\
6.5\end{array}$ & $\begin{array}{l}4.5 \\
4.0 \\
3.7 \\
3.5 \\
3.3 \\
---\end{array}$ & $\begin{array}{l}.11 \\
.15 \\
.14 \\
.34 \\
.38 \\
.43\end{array}$ & $\begin{array}{l}3.1 \\
2.6 \\
2.4 \\
2.7 \\
2.9 \\
2.6\end{array}$ \\
\hline
\end{tabular}




\begin{tabular}{|c|c|c|c|c|c|c|c|c|c|c|c|c|}
\hline Day & oct & lov & Dec & $\operatorname{Jan}$ & leb & Mar & $\overline{A p r}$ & May & $\operatorname{Jun} \theta$ & July & Aus & Sept \\
\hline \multicolumn{13}{|c|}{$\begin{array}{l}\text { Site 2--Eleventh Street Canal near Holly Hill } \\
\text { [Water year October } 1983 \text { to September 1984] }\end{array}$} \\
\hline $\begin{array}{l}1 \\
2 \\
3 \\
4 \\
5\end{array}$ & $\begin{array}{l}2.7 \\
2.5 \\
2.4 \\
2.5 \\
2.4\end{array}$ & $\begin{array}{l}3.5 \\
3.3 \\
3.1 \\
3.0 \\
3.0\end{array}$ & $\begin{array}{l}3.0 \\
2.8 \\
2.7 \\
2.5 \\
2.7\end{array}$ & $\begin{array}{l}--- \\
--- \\
--- \\
-- \\
---\end{array}$ & $\begin{array}{l}4.1 \\
3.9 \\
3.7 \\
3.6 \\
3.5\end{array}$ & $\begin{array}{l}4.6 \\
4.2 \\
4.2 \\
3.8 \\
3.7\end{array}$ & $\begin{array}{l}2.6 \\
2.6 \\
4.0 \\
64 \\
38\end{array}$ & $\begin{array}{l}3.2 \\
3.3 \\
3.2 \\
3.6 \\
7.3\end{array}$ & $\begin{array}{l}--- \\
--- \\
--- \\
-- \\
---\end{array}$ & $\begin{array}{l}6.1 \\
20 \\
27 \\
29 \\
19\end{array}$ & $\begin{array}{l}3.4 \\
3.2 \\
3.0 \\
2.9 \\
2.8\end{array}$ & $\begin{array}{l}2.6 \\
2.5 \\
2.4 \\
2.5 \\
4.2\end{array}$ \\
\hline $\begin{array}{r}6 \\
7 \\
8 \\
9 \\
10\end{array}$ & $\begin{array}{l}2.3 \\
2.3 \\
2.4 \\
3.3 \\
2.6\end{array}$ & $\begin{array}{l}2.9 \\
2.9 \\
3.1 \\
2.9 \\
2.9\end{array}$ & $\begin{array}{l}2.8 \\
2.5 \\
2.4 \\
2.6 \\
2.5\end{array}$ & $\begin{array}{l}--- \\
--- \\
--- \\
--\overline{9} \\
6.9\end{array}$ & $\begin{array}{l}3.4 \\
3.3 \\
3.3 \\
3.3 \\
3.1\end{array}$ & $\begin{array}{l}3.7 \\
3.5 \\
3.2 \\
3.2 \\
3.1\end{array}$ & $\begin{array}{r}19 \\
13 \\
9.7 \\
8.7 \\
8.6\end{array}$ & $\begin{array}{l}4.4 \\
3.7 \\
3.5 \\
3.4 \\
3.2\end{array}$ & $\begin{array}{l}--- \\
3.0 \\
2.9 \\
2.9 \\
2.8\end{array}$ & $\begin{array}{r}14 \\
11 \\
8.7 \\
7.3 \\
6.4\end{array}$ & $\begin{array}{l}2.7 \\
2.7 \\
2.6 \\
2.5 \\
2.5\end{array}$ & $\begin{array}{c}7.4 \\
19 \\
18 \\
41 \\
136\end{array}$ \\
\hline $\begin{array}{l}11 \\
12 \\
13 \\
14 \\
15\end{array}$ & $\begin{array}{l}2.9 \\
3.2 \\
2.8 \\
2.7 \\
2.6\end{array}$ & $\begin{array}{l}2.9 \\
2.9 \\
2.7 \\
2.7 \\
3.0\end{array}$ & $\begin{array}{l}2.8 \\
35 \\
12 \\
8.0 \\
7.2\end{array}$ & $\begin{array}{l}6.2 \\
5.6 \\
5.6 \\
5.4 \\
5.0\end{array}$ & $\begin{array}{l}3.1 \\
3.0 \\
4.7 \\
6.9 \\
5.2\end{array}$ & $\begin{array}{l}3.0 \\
3.1 \\
3.4 \\
3.4 \\
3.2\end{array}$ & $\begin{array}{l}7.5 \\
6.8 \\
6.3 \\
6.3 \\
9.6\end{array}$ & $\begin{array}{l}3.0 \\
2.9 \\
2.9 \\
2.8 \\
2.7\end{array}$ & $\begin{array}{l}2.9 \\
2.9 \\
2.8 \\
2.8 \\
2.7\end{array}$ & $\begin{array}{l}5.7 \\
5.2 \\
4.9 \\
4.6 \\
4.3\end{array}$ & $\begin{array}{l}2.4 \\
2.4 \\
2.4 \\
2.3 \\
2.3\end{array}$ & $\begin{array}{l}52 \\
24 \\
15 \\
12 \\
10\end{array}$ \\
\hline $\begin{array}{l}16 \\
17 \\
18 \\
19 \\
20\end{array}$ & $\begin{array}{l}5.6 \\
6.7 \\
6.5 \\
5.1 \\
4.3\end{array}$ & $\begin{array}{l}3.1 \\
3.0 \\
2.8 \\
2.7 \\
2.7\end{array}$ & $\begin{array}{r}6.3 \\
5.7 \\
8.1 \\
8.0 \\
37\end{array}$ & $\begin{array}{l}4.9 \\
4.8 \\
4.6 \\
5.6 \\
5.4\end{array}$ & $\begin{array}{l}4.7 \\
4.2 \\
3.8 \\
3.6 \\
3.5\end{array}$ & $\begin{array}{l}3.0 \\
3.0 \\
2.9 \\
2.8 \\
2.8\end{array}$ & $\begin{array}{l}8.1 \\
7.1 \\
6.2 \\
5.7 \\
5.4\end{array}$ & $\begin{array}{l}2.7 \\
2.6 \\
2.5 \\
2.4 \\
2.4\end{array}$ & $\begin{array}{l}2.6 \\
2.6 \\
3.2 \\
4.0 \\
3.3\end{array}$ & $\begin{array}{l}3.8 \\
3.5 \\
3.3 \\
3.4 \\
4.1\end{array}$ & $\begin{array}{l}2.2 \\
2.3 \\
2.5 \\
2.3 \\
2.6\end{array}$ & $\begin{array}{r}9.2 \\
8.6 \\
9.8 \\
11 \\
9.4\end{array}$ \\
\hline $\begin{array}{l}21 \\
22 \\
23 \\
24 \\
25\end{array}$ & $\begin{array}{r}3.9 \\
11 \\
9.2 \\
6.9 \\
5.5\end{array}$ & $\begin{array}{l}3.0 \\
2.9 \\
2.7 \\
2.5 \\
2.6\end{array}$ & $\begin{array}{l}77 \\
35 \\
19 \\
14 \\
10\end{array}$ & $\begin{array}{l}6.9 \\
6.6 \\
5.9 \\
5.9 \\
5.8\end{array}$ & $\begin{array}{l}3.6 \\
6.0 \\
7.2 \\
5.7 \\
5.0\end{array}$ & $\begin{array}{l}3.3 \\
2.9 \\
2.8 \\
2.8 \\
2.7\end{array}$ & $\begin{array}{l}5.0 \\
4.5 \\
4.4 \\
4.5 \\
4.3\end{array}$ & $\begin{array}{l}2.3 \\
2.3 \\
2.3 \\
-.- \\
---\end{array}$ & $\begin{array}{l}3.0 \\
2.9 \\
3.2 \\
4.0 \\
3.2\end{array}$ & $\begin{array}{l}5.6 \\
7.2 \\
7.7 \\
6.9 \\
5.9\end{array}$ & $\begin{array}{l}2.9 \\
2.9 \\
2.4 \\
2.4 \\
4.4\end{array}$ & $\begin{array}{l}8.1 \\
7.3 \\
6.8 \\
6.4 \\
5.9\end{array}$ \\
\hline $\begin{array}{l}26 \\
27 \\
28 \\
29 \\
30 \\
31\end{array}$ & $\begin{array}{l}4.8 \\
4.3 \\
3.9 \\
3.5 \\
3.3 \\
3.3\end{array}$ & $\begin{array}{l}2.4 \\
2.4 \\
2.4 \\
3.1 \\
3.3 \\
-.--\end{array}$ & $\begin{array}{c}9.0 \\
8.2 \\
7.8 \\
27 \\
41 \\
26\end{array}$ & $\begin{array}{l}5.7 \\
5.3 \\
4.7 \\
4.4 \\
4.4 \\
4.4\end{array}$ & $\begin{array}{l}4.4 \\
4.5 \\
6.0 \\
5.2 \\
-.- \\
---\end{array}$ & $\begin{array}{l}2.7 \\
2.9 \\
3.1 \\
2.8 \\
2.7 \\
2.7\end{array}$ & $\begin{array}{l}3.9 \\
3.6 \\
3.5 \\
3.4 \\
3.3 \\
--\end{array}$ & $\begin{array}{l}-- \\
--- \\
--- \\
-- \\
---\end{array}$ & $\begin{array}{l}3.2 \\
3.0 \\
2.9 \\
3.0 \\
3.8 \\
---\end{array}$ & $\begin{array}{l}5.0 \\
4.4 \\
4.0 \\
3.7 \\
3.5 \\
3.5\end{array}$ & $\begin{array}{l}5.6 \\
3.9 \\
3.3 \\
3.0 \\
2.8 \\
2.8\end{array}$ & $\begin{array}{l}5.6 \\
7.6 \\
56 \\
27 \\
16 \\
--\end{array}$ \\
\hline
\end{tabular}

Site 2--Eleventh Street Canal near Holly Hill [Water year October 1984 to September 1985]

\begin{tabular}{|c|c|c|c|c|c|c|c|c|c|c|c|c|}
\hline $\begin{array}{l}1 \\
2 \\
3 \\
4 \\
5\end{array}$ & $\begin{array}{c}13 \\
10 \\
9.1 \\
8.3 \\
7.5\end{array}$ & $\begin{array}{l}2.7 \\
2.8 \\
3.0 \\
3.1 \\
2.9\end{array}$ & $\begin{array}{l}3.7 \\
3.6 \\
3.5 \\
3.3 \\
3.8\end{array}$ & $\begin{array}{l}2.3 \\
2.3 \\
3.3 \\
3.4 \\
2.6\end{array}$ & $\begin{array}{l}1.9 \\
1.9 \\
1.9 \\
1.8 \\
1.8\end{array}$ & $\begin{array}{l}1.6 \\
1.6 \\
1.6 \\
1.6 \\
1.6\end{array}$ & $\begin{array}{l}1.5 \\
1.5 \\
1.5 \\
1.5 \\
1.5\end{array}$ & $\begin{array}{l}1.5 \\
1.4 \\
1.4 \\
2.7 \\
2.0\end{array}$ & $\begin{array}{l}1.4 \\
1.4 \\
1.4 \\
1.4 \\
1.4\end{array}$ & $\begin{array}{l}1.8 \\
1.7 \\
1.6 \\
1.6 \\
1.6\end{array}$ & $\begin{array}{l}3.3 \\
4.6 \\
3.7 \\
4.1 \\
3.4\end{array}$ & $\begin{array}{c}42 \\
57 \\
21 \\
12 \\
9.0\end{array}$ \\
\hline $\begin{array}{r}6 \\
7 \\
8 \\
9 \\
10\end{array}$ & $\begin{array}{l}6.9 \\
6.4 \\
6.3 \\
5.8 \\
5.5\end{array}$ & $\begin{array}{l}2.8 \\
2.7 \\
2.6 \\
2.6 \\
2.5\end{array}$ & $\begin{array}{l}3.7 \\
3.3 \\
3.2 \\
3.1 \\
3.0\end{array}$ & $\begin{array}{l}2.4 \\
2.4 \\
2.4 \\
2.3 \\
2.3\end{array}$ & $\begin{array}{l}2.0 \\
2.0 \\
2.0 \\
1.8 \\
1.8\end{array}$ & $\begin{array}{l}1.6 \\
1.6 \\
1.5 \\
1.5 \\
1.5\end{array}$ & $\begin{array}{l}1.5 \\
1.6 \\
1.5 \\
1.5 \\
1.4\end{array}$ & $\begin{array}{l}1.6 \\
1.5 \\
1.4 \\
1.4 \\
1.4\end{array}$ & $\begin{array}{l}1.3 \\
1.3 \\
1.3 \\
1.4 \\
1.4\end{array}$ & $\begin{array}{l}1.9 \\
1.9 \\
2.2 \\
1.9 \\
1.7\end{array}$ & $\begin{array}{c}3.0 \\
3.1 \\
8.7 \\
11 \\
9.1\end{array}$ & $\begin{array}{l}7.8 \\
7.5 \\
6.7 \\
6.0 \\
5.6\end{array}$ \\
\hline $\begin{array}{l}11 \\
12 \\
13 \\
14 \\
15\end{array}$ & $\begin{array}{l}5.3 \\
4.9 \\
4.6 \\
4.4 \\
4.1\end{array}$ & $\begin{array}{l}2.5 \\
2.4 \\
2.4 \\
2.4 \\
2.3\end{array}$ & $\begin{array}{l}3.0 \\
2.9 \\
2.9 \\
2.8 \\
2.7\end{array}$ & $\begin{array}{l}2.2 \\
2.2 \\
2.2 \\
2.2 \\
2.1\end{array}$ & $\begin{array}{l}1.8 \\
1.9 \\
1.8 \\
1.8 \\
1.8\end{array}$ & $\begin{array}{l}1.5 \\
1.5 \\
1.5 \\
1.5 \\
1.5\end{array}$ & $\begin{array}{l}1.4 \\
1.4 \\
2.7 \\
1.9 \\
1.6\end{array}$ & $\begin{array}{l}1.4 \\
1.4 \\
1.4 \\
1.4 \\
1.4\end{array}$ & $\begin{array}{l}1.4 \\
1.4 \\
1.8 \\
3.3 \\
4.1\end{array}$ & $\begin{array}{l}1.7 \\
1.7 \\
1.8 \\
1.7 \\
1.7\end{array}$ & $\begin{array}{l}12 \\
17 \\
10 \\
9.0 \\
9.4\end{array}$ & $\begin{array}{l}5.2 \\
4.9 \\
4.8 \\
5.3 \\
5.0\end{array}$ \\
\hline $\begin{array}{l}16 \\
17 \\
18 \\
19 \\
20\end{array}$ & $\begin{array}{l}3.9 \\
3.8 \\
3.6 \\
3.5 \\
3.4\end{array}$ & $\begin{array}{l}2.3 \\
2.2 \\
2.2 \\
2.2 \\
2.2\end{array}$ & $\begin{array}{l}2.7 \\
2.7 \\
2.7 \\
2.9 \\
2.9\end{array}$ & $\begin{array}{l}2.1 \\
2.1 \\
2.1 \\
2.1 \\
2.1\end{array}$ & $\begin{array}{l}1.8 \\
1.8 \\
1.8 \\
1.8 \\
1.8\end{array}$ & $\begin{array}{l}1.5 \\
1.7 \\
1.6 \\
1.5 \\
1.5\end{array}$ & $\begin{array}{l}1.6 \\
1.5 \\
1.5 \\
1.5 \\
1.5\end{array}$ & $\begin{array}{l}1.4 \\
1.4 \\
1.4 \\
1.4 \\
1.5\end{array}$ & $\begin{array}{l}3.5 \\
3.0 \\
2.5 \\
2.1 \\
2.0\end{array}$ & $\begin{array}{l}1.8 \\
2.6 \\
2.0 \\
1.8 \\
1.8\end{array}$ & $\begin{array}{l}8.3 \\
6.9 \\
6.1 \\
5.6 \\
5.2\end{array}$ & $\begin{array}{r}4.6 \\
5.7 \\
7.5 \\
8.1 \\
21\end{array}$ \\
\hline $\begin{array}{l}21 \\
22 \\
23 \\
24 \\
25\end{array}$ & $\begin{array}{l}3.9 \\
4.0 \\
3.5 \\
3.4 \\
3.2\end{array}$ & $\begin{array}{l}2.2 \\
3.3 \\
8.4 \\
8.2 \\
5.8\end{array}$ & $\begin{array}{l}2.8 \\
2.7 \\
2.6 \\
2.6 \\
2.6\end{array}$ & $\begin{array}{l}2.1 \\
2.1 \\
2.1 \\
2.1 \\
2.0\end{array}$ & $\begin{array}{l}1.7 \\
1.7 \\
1.7 \\
1.7 \\
1.7\end{array}$ & $\begin{array}{l}1.6 \\
2.4 \\
1.7 \\
1.6 \\
1.6\end{array}$ & $\begin{array}{l}1.5 \\
1.5 \\
1.4 \\
1.4 \\
1.4\end{array}$ & $\begin{array}{l}2.3 \\
1.5 \\
1.6 \\
2.3 \\
1.6\end{array}$ & $\begin{array}{l}1.9 \\
1.8 \\
1.8 \\
1.7 \\
1.7\end{array}$ & $\begin{array}{r}1.7 \\
1.7 \\
1.7 \\
6.7 \\
15\end{array}$ & $\begin{array}{l}4.9 \\
4.7 \\
4.7 \\
4.3 \\
4.0\end{array}$ & $\begin{array}{l}58 \\
40 \\
19 \\
13 \\
11\end{array}$ \\
\hline $\begin{array}{l}26 \\
27 \\
28 \\
29 \\
30 \\
31\end{array}$ & $\begin{array}{l}3.1 \\
3.1 \\
3.0 \\
2.9 \\
2.9 \\
2.9\end{array}$ & $\begin{array}{l}5.1 \\
4.7 \\
4.4 \\
4.2 \\
3.9 \\
. .-\end{array}$ & $\begin{array}{l}2.5 \\
2.5 \\
2.5 \\
2.4 \\
2.4 \\
2.3\end{array}$ & $\begin{array}{l}2.0 \\
2.0 \\
2.0 \\
2.0 \\
1.9 \\
1.9\end{array}$ & \begin{tabular}{l}
1.7 \\
1.6 \\
1.6 \\
\hdashline- \\
\hdashline$\ldots$
\end{tabular} & $\begin{array}{l}1.5 \\
1.5 \\
1.5 \\
1.5 \\
1.5 \\
1.5\end{array}$ & $\begin{array}{l}1.4 \\
1.4 \\
1.4 \\
1.4 \\
1.5 \\
-\end{array}$ & $\begin{array}{l}1.5 \\
1.5 \\
1.4 \\
1.4 \\
1.4 \\
1.4\end{array}$ & $\begin{array}{l}1.7 \\
1.6 \\
1.9 \\
2.1 \\
1.9 \\
-\end{array}$ & $\begin{array}{l}7.2 \\
5.2 \\
4.3 \\
3.7 \\
3.3 \\
3.0\end{array}$ & $\begin{array}{l}3.7 \\
3.9 \\
4.5 \\
4.2 \\
4.1 \\
5.9\end{array}$ & $\begin{array}{l}9.1 \\
8.3 \\
8.0 \\
7.5 \\
7.1 \\
-\end{array}$ \\
\hline
\end{tabular}


APPENDIX III--Daily discharge--Continued

[Mean values in cubic feet per second]

\begin{tabular}{|c|c|c|c|c|c|c|c|c|c|c|c|c|}
\hline Day & Oct & Iov & Dec & $\operatorname{Jan}$ & Fबb & Mar & Apr & May & June & July & Aug & Sept \\
\hline \multicolumn{13}{|c|}{$\begin{array}{l}\text { Site 3--Williamson Boulevard ditch at Daytona Beach } \\
\text { [Water year October } 1982 \text { to September 1983] }\end{array}$} \\
\hline $\begin{array}{l}1 \\
2 \\
3 \\
4 \\
5\end{array}$ & $\begin{array}{l}\cdots \\
\cdots \\
\cdots \\
\cdots-\end{array}$ & \begin{tabular}{l}
$-\ldots$ \\
$-\cdots$ \\
\hdashline-- \\
$-\cdots$
\end{tabular} & \begin{tabular}{l}
$\overline{-}$ \\
$\overline{-}$ \\
\hdashline- \\
--
\end{tabular} & \begin{tabular}{l}
$-\cdots$ \\
$\overline{-}$ \\
\hdashline-- \\
--
\end{tabular} & \begin{tabular}{l}
--- \\
\hdashline- \\
\hdashline- \\
---
\end{tabular} & $\begin{array}{l}--- \\
\overline{--} \\
--- \\
---\end{array}$ & \begin{tabular}{l}
-- \\
-- \\
\hdashline- \\
--
\end{tabular} & $\begin{array}{l}-\cdots \\
\cdots- \\
\cdots- \\
\cdots-\end{array}$ & \begin{tabular}{l}
-- \\
$\cdots-$ \\
\hdashline- \\
-
\end{tabular} & $\begin{array}{r}0.46 \\
.44 \\
.41 \\
.39 \\
.47\end{array}$ & $\begin{array}{c}0.12 \\
.0 \\
.0 \\
.0 \\
.0\end{array}$ & $\begin{array}{c}0.51 \\
.86 \\
1.2 \\
1.2 \\
.69\end{array}$ \\
\hline $\begin{array}{r}6 \\
7 \\
8 \\
9 \\
10\end{array}$ & $\begin{array}{l}-.- \\
\overline{-} \\
\overline{-} \\
\overline{-}\end{array}$ & $\begin{array}{l}--- \\
-\cdots \\
-\cdots \\
-\cdots\end{array}$ & $\begin{array}{l}\cdots- \\
\cdots- \\
\cdots- \\
\cdots-\end{array}$ & $\begin{array}{l}-\cdots \\
\overline{-} \\
\overline{-} \\
-\cdots\end{array}$ & \begin{tabular}{l}
$\cdots-$ \\
\hdashline- \\
\hdashline- \\
\hdashline- \\
\end{tabular} & $\begin{array}{l}--- \\
--- \\
--- \\
-\cdots\end{array}$ & $\begin{array}{l}-- \\
- \\
- \\
- \\
-\end{array}$ & $\begin{array}{l}\cdots- \\
\cdots- \\
\cdots- \\
\cdots\end{array}$ & $\begin{array}{l}--- \\
2.3 \\
1.6 \\
1.1\end{array}$ & $\begin{array}{r}.41 \\
.35 \\
.43 \\
.39 \\
.28\end{array}$ & $\begin{array}{l}.0 \\
.10 \\
.06 \\
.84 \\
.84\end{array}$ & $\begin{array}{l}.45 \\
.33 \\
.27 \\
.25 \\
.21\end{array}$ \\
\hline $\begin{array}{l}11 \\
12 \\
13 \\
14 \\
15\end{array}$ & $\overline{-}$ & $\begin{array}{l}-\cdots \\
\cdots- \\
\cdots-\end{array}$ & $\begin{array}{c}-- \\
\cdots \\
\cdots \\
\cdots\end{array}$ & \begin{tabular}{l}
$-\cdots$ \\
$\overline{-}$ \\
\hdashline- \\
$-\cdots$
\end{tabular} & $\begin{array}{l}\cdots \\
\cdots- \\
\cdots- \\
\cdots-\end{array}$ & \begin{tabular}{l}
--- \\
$-\cdots$ \\
\hdashline- \\
$-\cdots$
\end{tabular} & $\begin{array}{l}-- \\
- \\
- \\
-\end{array}$ & $\begin{array}{l}-\cdots \\
-\cdots \\
-\cdots \\
-\cdots\end{array}$ & $\begin{array}{l}.84 \\
.69 \\
.75 \\
.59 \\
.46\end{array}$ & $\begin{array}{l}.22 \\
.21 \\
.23 \\
.23 \\
.20\end{array}$ & $\begin{array}{r}.43 \\
.37 \\
1.2 \\
1.6 \\
1.4\end{array}$ & $\begin{array}{l}.10 \\
.09 \\
.03 \\
.09 \\
.11\end{array}$ \\
\hline $\begin{array}{l}16 \\
17 \\
18 \\
19 \\
20\end{array}$ & ב- & $\begin{array}{l}-\cdots \\
-\cdots \\
-\cdots \\
-\cdots\end{array}$ & $\begin{array}{l}\cdots \\
\cdots \\
\cdots- \\
\cdots-\end{array}$ & $\begin{array}{l}\cdots \\
\cdots \\
\cdots- \\
\cdots\end{array}$ & $\begin{array}{l}-\cdots \\
-\cdots \\
-\cdots \\
---\end{array}$ & $\begin{array}{l}-\cdots \\
-\cdots \\
-\cdots \\
-\cdots\end{array}$ & ב- & $\begin{array}{l}--- \\
--- \\
--- \\
---\end{array}$ & $\begin{array}{l}.41 \\
.36 \\
.35 \\
.34 \\
.35\end{array}$ & $\begin{array}{l}.16 \\
.13 \\
.10 \\
.11 \\
.10\end{array}$ & $\begin{array}{l}.88 \\
.64 \\
.51 \\
.42 \\
.36\end{array}$ & $\begin{array}{l}.04 \\
.41 \\
.42 \\
.43 \\
1.1\end{array}$ \\
\hline $\begin{array}{l}21 \\
22 \\
23 \\
24 \\
25\end{array}$ & $\begin{array}{l}-\cdots \\
\cdots \\
\cdots \\
-\cdots\end{array}$ & \begin{tabular}{l}
--- \\
$-\cdots$ \\
\hdashline- \\
$-\cdots$
\end{tabular} & $\begin{array}{l}\cdots- \\
\cdots- \\
\cdots \\
\cdots-\end{array}$ & \begin{tabular}{l}
$-\cdots$ \\
$\cdots-$ \\
$\cdots-$ \\
\hdashline-
\end{tabular} & \begin{tabular}{l}
$-\cdots$ \\
$\cdots-$ \\
\hdashline- \\
$-\cdots$
\end{tabular} & $\begin{array}{l}-\cdots \\
--- \\
-\cdots \\
-\cdots\end{array}$ & $\begin{array}{l}-- \\
- \\
- \\
-\end{array}$ & $\begin{array}{l}--- \\
-\cdots \\
-\cdots \\
---\end{array}$ & $\begin{array}{l}.85 \\
1.5 \\
1.6 \\
1.8 \\
1.2\end{array}$ & $\begin{array}{l}.06 \\
.06 \\
.07 \\
.05 \\
.02\end{array}$ & $\begin{array}{l}.31 \\
.26 \\
.19 \\
.14 \\
.31\end{array}$ & $\begin{array}{r}.81 \\
.63 \\
.44 \\
.36 \\
1.2\end{array}$ \\
\hline $\begin{array}{l}26 \\
27 \\
28 \\
29 \\
30 \\
31\end{array}$ & 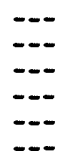 & \begin{tabular}{l}
$-\cdots$ \\
$\cdots-$ \\
\hdashline- \\
\hdashline-
\end{tabular} & 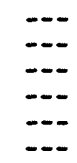 & $\overline{-}$ & \begin{tabular}{l}
.-- \\
\hdashline- \\
\hdashline- \\
\hdashline- \\
$-\cdots$
\end{tabular} & $\begin{array}{l}-- \\
\cdots- \\
--- \\
-\cdots\end{array}$ & $\overline{-}$ & $\begin{array}{l}-- \\
\cdots- \\
\cdots- \\
-\cdots \\
-\cdots\end{array}$ & $\begin{array}{l}.88 \\
.62 \\
.41 \\
.40 \\
.42 \\
-.-\end{array}$ & $\begin{array}{l}.05 \\
.04 \\
.0 \\
.21 \\
.04 \\
.32\end{array}$ & $\begin{array}{l}.24 \\
.04 \\
.12 \\
.10 \\
.02 \\
.01\end{array}$ & $\begin{array}{l}.89 \\
.69 \\
.50 \\
.40 \\
.34 \\
-\end{array}$ \\
\hline
\end{tabular}

Site 3--Williamson Boulevard ditch at Daytona Beach [Water year October 1983 to September 1984]

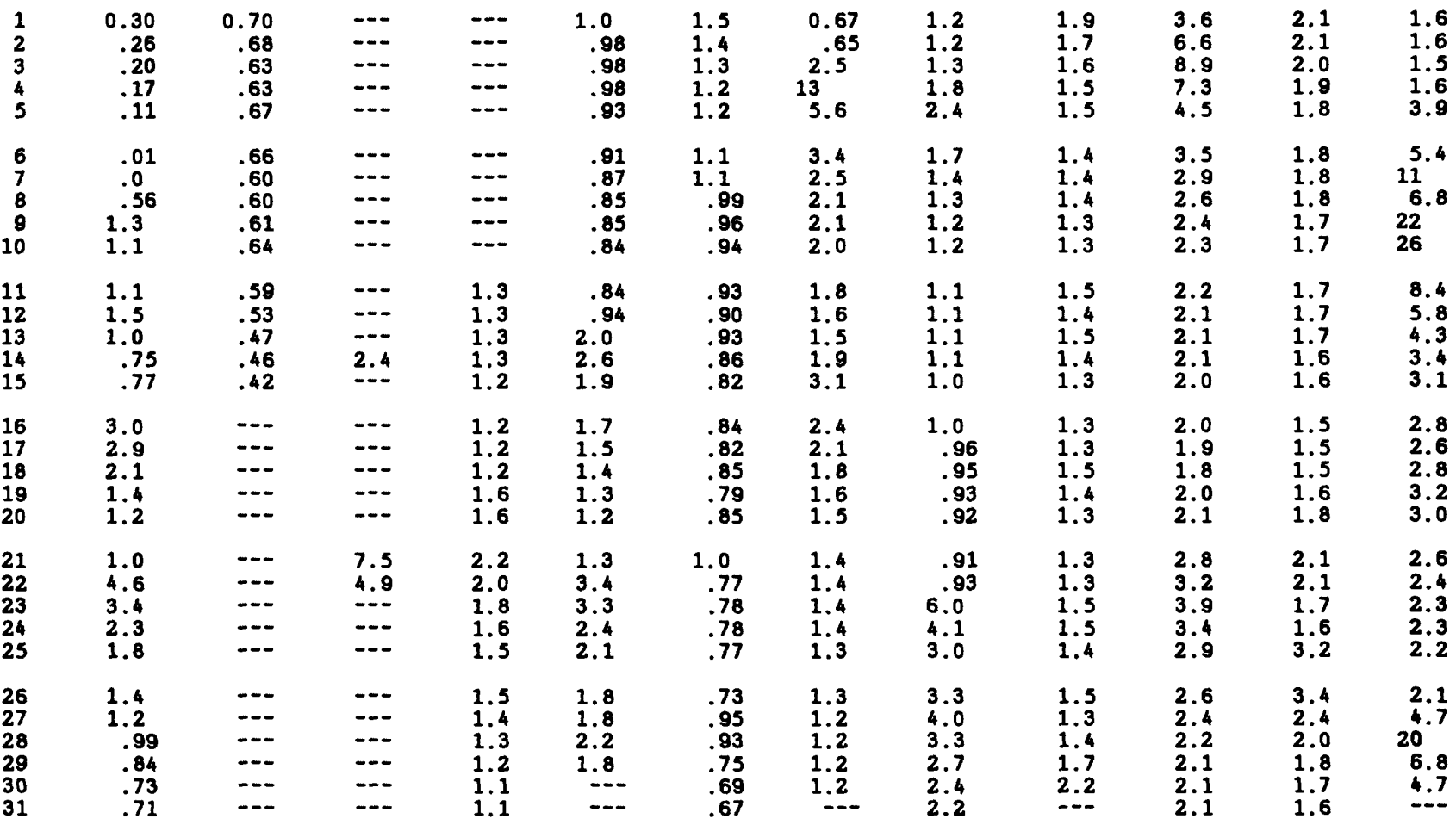




\begin{tabular}{|c|c|c|c|c|c|c|c|c|c|c|c|c|}
\hline Day & Oct & Nov & Dec & Jan & Feb & Mar & Apr & May & June & July & Aug & Sept \\
\hline \multicolumn{13}{|c|}{$\begin{array}{l}\text { Site 3--Williamson Boulevard ditch at Daytona Beach } \\
\text { [Water year October 1984 to September 1985] }\end{array}$} \\
\hline $\begin{array}{l}11 \\
12 \\
13 \\
14 \\
15\end{array}$ & $\begin{array}{l}2.3 \\
2.3 \\
2.2 \\
2.2 \\
2.2\end{array}$ & $\begin{array}{l}2.0 \\
1.9 \\
1.9 \\
1.9 \\
1.9\end{array}$ & $\begin{array}{l}2.0 \\
2.0 \\
2.0 \\
2.0 \\
1.9\end{array}$ & $\begin{array}{l}1.8 \\
1.8 \\
1.8 \\
1.8 \\
1.8\end{array}$ & $\begin{array}{l}1.8 \\
1.7 \\
1.7 \\
1.7 \\
1.7\end{array}$ & $\begin{array}{l}1.5 \\
1.4 \\
1.4 \\
1.4 \\
1.4\end{array}$ & $\begin{array}{l}1.2 \\
1.2 \\
1.8 \\
1.5 \\
1.5\end{array}$ & $\begin{array}{l}1.1 \\
1.1 \\
1.1 \\
1.1 \\
1.1\end{array}$ & $\begin{array}{l}1.2 \\
1.4 \\
1.8 \\
2.3 \\
4.2\end{array}$ & $\begin{array}{l}1.9 \\
2.3 \\
2.6 \\
2.4 \\
2.2\end{array}$ & $\begin{array}{r}11 \\
9.5 \\
5.8 \\
5.4 \\
5.3\end{array}$ & $\begin{array}{l}3.4 \\
3.2 \\
3.2 \\
3.4 \\
3.1\end{array}$ \\
\hline $\begin{array}{l}21 \\
22 \\
23 \\
24 \\
25\end{array}$ & $\begin{array}{l}2.2 \\
2.2 \\
2.1 \\
2.1 \\
2.1\end{array}$ & $\begin{array}{l}1.9 \\
2.5 \\
4.6 \\
3.7 \\
2.8\end{array}$ & $\begin{array}{l}1.8 \\
1.8 \\
1.8 \\
1.8 \\
1.8\end{array}$ & $\begin{array}{l}1.7 \\
1.7 \\
1.8 \\
1.8 \\
1.8\end{array}$ & $\begin{array}{l}1.7 \\
1.6 \\
1.6 \\
1.6 \\
1.6\end{array}$ & $\begin{array}{l}1.7 \\
1.7 \\
1.6 \\
1.4 \\
1.4\end{array}$ & $\begin{array}{l}1.2 \\
1.1 \\
1.2 \\
1.1 \\
1.1\end{array}$ & $\begin{array}{l}1.7 \\
1.4 \\
2.1 \\
2.7 \\
2.0\end{array}$ & $\begin{array}{l}2.0 \\
2.0 \\
1.9 \\
1.8 \\
1.8\end{array}$ & $\begin{array}{l}2.1 \\
2.1 \\
2.1 \\
3.5 \\
5.1\end{array}$ & $\begin{array}{l}3.5 \\
3.5 \\
3.6 \\
3.3 \\
3.1\end{array}$ & $\begin{array}{c}25 \\
10 \\
6.6 \\
5.2 \\
4.6\end{array}$ \\
\hline $\begin{array}{l}26 \\
27 \\
28 \\
29 \\
30 \\
31\end{array}$ & $\begin{array}{l}2.1 \\
2.1 \\
2.1 \\
2.0 \\
2.0 \\
2.0\end{array}$ & $\begin{array}{l}2.7 \\
2.6 \\
2.5 \\
2.4 \\
2.3 \\
-.-\end{array}$ & $\begin{array}{l}1.8 \\
1.8 \\
1.8 \\
1.8 \\
1.8 \\
1.8\end{array}$ & $\begin{array}{l}1.7 \\
1.8 \\
1.8 \\
1.8 \\
1.8 \\
1.8\end{array}$ & $\begin{array}{l}1.6 \\
1.5 \\
1.5 \\
-- \\
---\end{array}$ & $\begin{array}{l}1.4 \\
1.3 \\
1.4 \\
1.4 \\
1.3 \\
1.3\end{array}$ & $\begin{array}{l}1.2 \\
1.1 \\
1.1 \\
1.0 \\
1.2 \\
-\end{array}$ & $\begin{array}{l}1.7 \\
1.6 \\
1.5 \\
1.5 \\
1.5 \\
1.5\end{array}$ & $\begin{array}{l}1.8 \\
1.8 \\
1.9 \\
2.0 \\
2.0 \\
\end{array}$ & $\begin{array}{l}3.6 \\
3.0 \\
2.8 \\
2.6 \\
2.5 \\
2.5\end{array}$ & $\begin{array}{l}2.9 \\
3.9 \\
4.4 \\
3.7 \\
3.6 \\
8.4\end{array}$ & $\begin{array}{r}4.3 \\
4.0 \\
6.6 \\
5.4 \\
4.7 \\
\end{array}$ \\
\hline
\end{tabular}

Site 4--Wally Boffmeyer Canal at Daytona Beach [Water year October 1982 to September 1983]

\begin{tabular}{|c|c|c|c|c|c|c|c|c|c|c|c|c|}
\hline $\begin{array}{l}1 \\
2 \\
3 \\
4 \\
5\end{array}$ & $\begin{array}{l}-- \\
-- \\
-- \\
--\end{array}$ & $\begin{array}{l}--- \\
--- \\
--- \\
--- \\
---\end{array}$ & $\begin{array}{l}2.0 \\
2.0 \\
1.9 \\
1.9 \\
2.0\end{array}$ & $\begin{array}{l}1.9 \\
1.8 \\
1.9 \\
1.9 \\
1.7\end{array}$ & $\begin{array}{r}1.6 \\
20 \\
4.3 \\
3.4 \\
2.8\end{array}$ & $\begin{array}{l}3.6 \\
3.2 \\
3.0 \\
3.0 \\
3.0\end{array}$ & $\begin{array}{l}4.3 \\
3.6 \\
3.4 \\
2.8 \\
2.6\end{array}$ & $\begin{array}{l}2.4 \\
2.3 \\
2.3 \\
2.9 \\
2.6\end{array}$ & $\begin{array}{l}2.7 \\
2.3 \\
2.1 \\
2.2 \\
5.0\end{array}$ & $\begin{array}{l}2.3 \\
2.1 \\
2.0 \\
1.9 \\
1.9\end{array}$ & $\begin{array}{l}2.5 \\
2.2 \\
1.9 \\
2.2 \\
2.0\end{array}$ & $\begin{array}{l}4.4 \\
7.7 \\
5.9 \\
3.6 \\
2.6\end{array}$ \\
\hline $\begin{array}{r}6 \\
7 \\
8 \\
9 \\
10\end{array}$ & $\begin{array}{l}--- \\
--- \\
--- \\
---\end{array}$ & $\begin{array}{l}--- \\
--- \\
--- \\
--- \\
---\end{array}$ & $\begin{array}{l}1.9 \\
3.1 \\
3.0 \\
2.4 \\
5.7\end{array}$ & $\begin{array}{l}1.6 \\
1.7 \\
1.6 \\
1.6 \\
1.6\end{array}$ & $\begin{array}{l}5.1 \\
3.7 \\
2.8 \\
2.8 \\
3.9\end{array}$ & $\begin{array}{r}3.0 \\
12 \\
6.0 \\
4.0 \\
3.5\end{array}$ & $\begin{array}{c}2.6 \\
7.6 \\
12 \\
7.9 \\
13\end{array}$ & $\begin{array}{l}2.3 \\
2.2 \\
2.2 \\
2.2 \\
2.1\end{array}$ & $\begin{array}{c}3.9 \\
15 \\
16 \\
4.7 \\
3.5\end{array}$ & $\begin{array}{l}2.0 \\
1.9 \\
1.9 \\
1.9 \\
1.8\end{array}$ & $\begin{array}{c}1.6 \\
2.1 \\
2.5 \\
12 \\
9.3\end{array}$ & $\begin{array}{l}2.3 \\
2.2 \\
2.1 \\
2.0 \\
2.0\end{array}$ \\
\hline $\begin{array}{l}11 \\
12 \\
13 \\
14 \\
15\end{array}$ & $\begin{array}{l}--- \\
--- \\
--- \\
---\end{array}$ & $\begin{array}{l}--- \\
--- \\
--- \\
--- \\
--\end{array}$ & $\begin{array}{l}3.3 \\
2.7 \\
2.4 \\
2.0 \\
1.9\end{array}$ & $\begin{array}{l}1.6 \\
1.6 \\
1.6 \\
1.6 \\
1.6\end{array}$ & $\begin{array}{r}3.7 \\
4.2 \\
25 \\
8.1 \\
4.9\end{array}$ & $\begin{array}{l}3.2 \\
3.2 \\
2.9 \\
2.8 \\
8.8\end{array}$ & $\begin{array}{l}5.9 \\
4.2 \\
3.4 \\
3.3 \\
9.0\end{array}$ & $\begin{array}{l}2.1 \\
2.1 \\
2.0 \\
2.0 \\
2.0\end{array}$ & $\begin{array}{l}3.0 \\
2.7 \\
6.0 \\
3.5 \\
2.7\end{array}$ & $\begin{array}{l}1.7 \\
1.7 \\
1.7 \\
1.7 \\
1.7\end{array}$ & $\begin{array}{l}2.9 \\
3.1 \\
8.0 \\
9.5 \\
5.9\end{array}$ & $\begin{array}{l}1.9 \\
1.9 \\
1.9 \\
1.9 \\
2.0\end{array}$ \\
\hline $\begin{array}{l}16 \\
17 \\
18 \\
19 \\
20\end{array}$ & $\begin{array}{l}--- \\
--- \\
-- \\
--\end{array}$ & $\begin{array}{l}--- \\
--- \\
--- \\
---\end{array}$ & $\begin{array}{l}2.0 \\
2.5 \\
2.0 \\
2.0 \\
1.9\end{array}$ & $\begin{array}{l}1.6 \\
1.6 \\
1.6 \\
1.6 \\
7.0\end{array}$ & $\begin{array}{l}5.5 \\
5.7 \\
4.4 \\
3.8 \\
3.6\end{array}$ & $\begin{array}{c}21 \\
26 \\
9.8 \\
5.1 \\
4.1\end{array}$ & $\begin{array}{l}6.6 \\
3.9 \\
3.7 \\
7.5 \\
4.6\end{array}$ & $\begin{array}{l}2.0 \\
2.0 \\
1.9 \\
2.0 \\
2.0\end{array}$ & $\begin{array}{l}2.5 \\
2.4 \\
2.2 \\
2.1 \\
2.1\end{array}$ & $\begin{array}{l}1.7 \\
1.6 \\
1.6 \\
1.6 \\
1.6\end{array}$ & $\begin{array}{l}3.4 \\
2.8 \\
2.5 \\
2.3 \\
2.2\end{array}$ & $\begin{array}{l}1.8 \\
3.5 \\
3.4 \\
3.6 \\
5.9\end{array}$ \\
\hline $\begin{array}{l}21 \\
22 \\
23 \\
24 \\
25\end{array}$ & $\begin{array}{l}--- \\
--- \\
--- \\
---\end{array}$ & $\begin{array}{l}--- \\
--- \\
--- \\
--- \\
---\end{array}$ & $\begin{array}{l}1.8 \\
1.7 \\
1.8 \\
1.7 \\
1.7\end{array}$ & $\begin{array}{l}4.6 \\
2.6 \\
2.5 \\
2.3 \\
2.1\end{array}$ & $\begin{array}{l}3.4 \\
3.5 \\
3.2 \\
3.3 \\
3.3\end{array}$ & $\begin{array}{l}5.9 \\
2.9 \\
4.4 \\
5.0 \\
3.5\end{array}$ & $\begin{array}{l}3.4 \\
3.0 \\
7.0 \\
5.7 \\
3.8\end{array}$ & $\begin{array}{l}1.9 \\
1.8 \\
1.8 \\
1.9 \\
1.8\end{array}$ & $\begin{array}{l}5.0 \\
6.4 \\
4.1 \\
4.7 \\
3.0\end{array}$ & $\begin{array}{l}1.6 \\
1.6 \\
1.6 \\
1.6 \\
1.6\end{array}$ & $\begin{array}{l}2.1 \\
2.0 \\
2.0 \\
1.9 \\
2.3\end{array}$ & $\begin{array}{l}3.0 \\
2.6 \\
2.2 \\
2.1 \\
8.7\end{array}$ \\
\hline $\begin{array}{l}26 \\
27 \\
28 \\
29 \\
30 \\
31\end{array}$ & $\begin{array}{l}--- \\
-- \\
-- \\
-- \\
--- \\
--\end{array}$ & $\begin{array}{l}--- \\
--- \\
--- \\
--0 \\
---\end{array}$ & $\begin{array}{l}1.7 \\
1.7 \\
1.7 \\
1.7 \\
2.0 \\
2.1\end{array}$ & $\begin{array}{l}2.1 \\
2.7 \\
3.0 \\
2.2 \\
2.1 \\
1.9\end{array}$ & $\begin{array}{l}3.1 \\
3.1 \\
3.2 \\
--- \\
---\end{array}$ & $\begin{array}{l}3.0 \\
4.2 \\
4.1 \\
3.2 \\
2.9 \\
9.3\end{array}$ & $\begin{array}{l}3.1 \\
2.8 \\
2.6 \\
2.5 \\
2.4 \\
--\end{array}$ & $\begin{array}{l}2.1 \\
1.8 \\
1.7 \\
6.4 \\
9.3\end{array}$ & $\begin{array}{l}2.5 \\
2.3 \\
2.2 \\
2.1 \\
2.1 \\
-. .\end{array}$ & $\begin{array}{l}2.4 \\
2.4 \\
1.6 \\
3.3 \\
2.0 \\
3.6\end{array}$ & $\begin{array}{l}2.4 \\
1.9 \\
2.0 \\
2.4 \\
1.9 \\
1.8\end{array}$ & $\begin{array}{l}3.2 \\
2.8 \\
2.4 \\
2.2 \\
2.1\end{array}$ \\
\hline
\end{tabular}


APPENDIX III--Daily discharge--Continued

[Mean values in cubic feet per second]

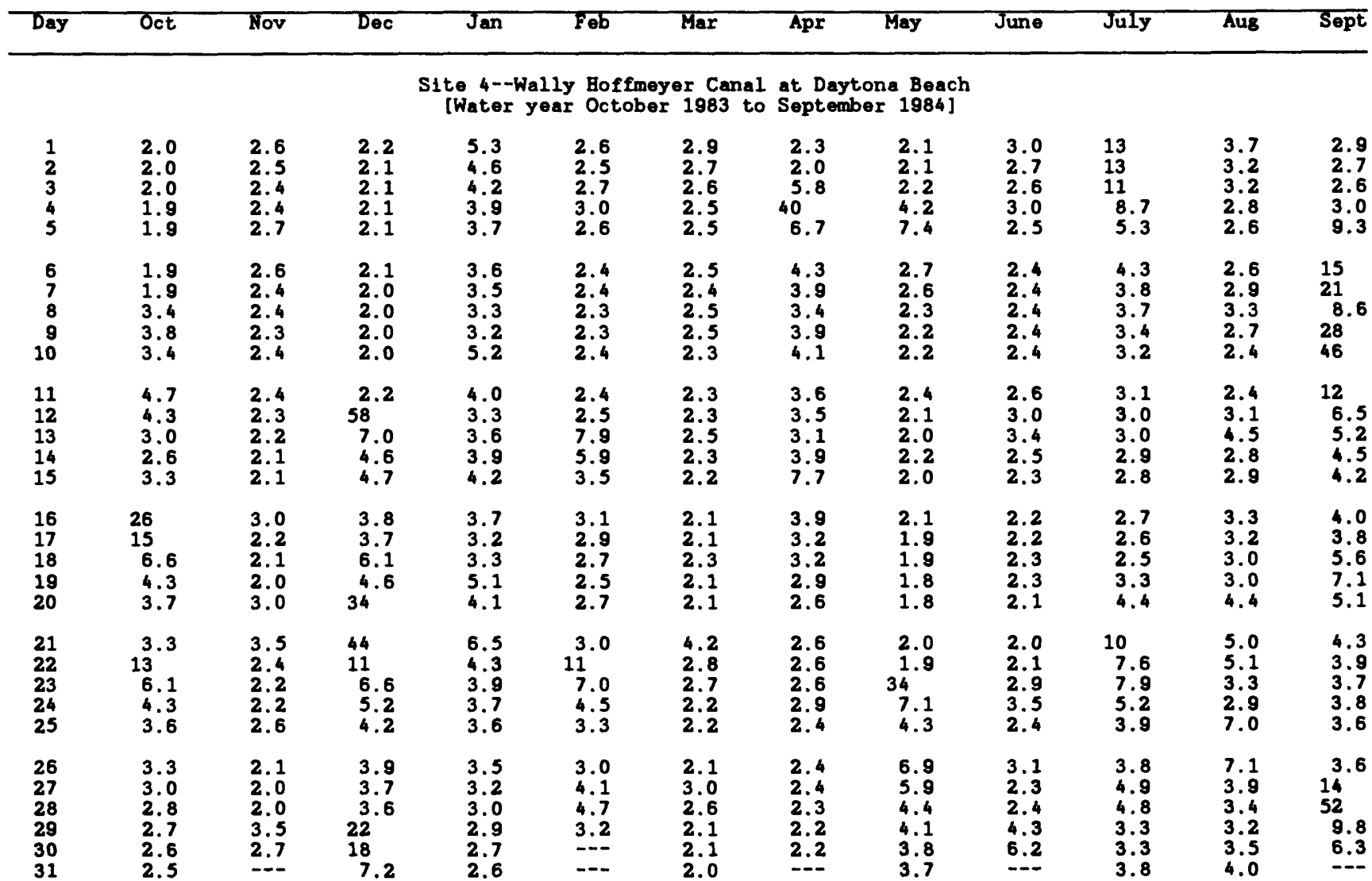

Site 4--Wally Hoffmeyer Canal at Daytona Beach [Water year October 1984 to September 1985]

\begin{tabular}{|c|c|c|c|c|c|c|c|c|c|c|c|c|}
\hline $\begin{array}{l}1 \\
2 \\
3 \\
4 \\
5\end{array}$ & $\begin{array}{l}5.3 \\
4.5 \\
4.1 \\
4.3 \\
3.8\end{array}$ & $\begin{array}{l}2.8 \\
2.8 \\
3.0 \\
3.5 \\
2.9\end{array}$ & $\begin{array}{l}3.2 \\
3.1 \\
3.2 \\
3.2 \\
3.4\end{array}$ & $\begin{array}{l}2.4 \\
2.4 \\
5.5 \\
5.0 \\
3.1\end{array}$ & $\begin{array}{l}2.2 \\
2.1 \\
2.1 \\
2.1 \\
2.1\end{array}$ & $\begin{array}{l}1.8 \\
1.8 \\
1.8 \\
1.8 \\
1.8\end{array}$ & $\begin{array}{l}1.7 \\
1.9 \\
1.7 \\
1.6 \\
1.6\end{array}$ & $\begin{array}{l}2.3 \\
1.9 \\
1.8 \\
9.9 \\
5.9\end{array}$ & $\begin{array}{l}2.0 \\
2.0 \\
2.0 \\
2.0 \\
1.9\end{array}$ & $\begin{array}{l}3.4 \\
2.7 \\
2.4 \\
2.4 \\
2.4\end{array}$ & $\begin{array}{l}6.3 \\
7.8 \\
4.8 \\
6.6 \\
3.9\end{array}$ & $\begin{array}{l}55 \\
28 \\
8.9 \\
6.4 \\
5.7\end{array}$ \\
\hline $\begin{array}{r}6 \\
7 \\
8 \\
9 \\
10\end{array}$ & $\begin{array}{l}3.7 \\
3.7 \\
3.6 \\
3.5 \\
3.8\end{array}$ & $\begin{array}{l}2.7 \\
2.6 \\
2.5 \\
2.5 \\
2.5\end{array}$ & $\begin{array}{l}3.3 \\
3.0 \\
2.9 \\
2.8 \\
2.9\end{array}$ & $\begin{array}{l}2.7 \\
2.6 \\
2.5 \\
2.5 \\
2.4\end{array}$ & $\begin{array}{l}2.4 \\
2.6 \\
2.6 \\
2.2 \\
2.0\end{array}$ & $\begin{array}{l}1.8 \\
1.8 \\
1.7 \\
1.7 \\
1.7\end{array}$ & $\begin{array}{l}2.0 \\
3.0 \\
2.0 \\
1.7 \\
1.7\end{array}$ & $\begin{array}{l}3.3 \\
2.6 \\
2.4 \\
2.3 \\
2.3\end{array}$ & $\begin{array}{l}2.0 \\
2.2 \\
2.0 \\
3.7 \\
2.2\end{array}$ & $\begin{array}{l}4.0 \\
3.3 \\
5.2 \\
3.0 \\
2.6\end{array}$ & $\begin{array}{c}3.6 \\
6.1 \\
19 \\
11 \\
6.4\end{array}$ & $\begin{array}{l}6.7 \\
6.9 \\
5.4 \\
4.9 \\
5.1\end{array}$ \\
\hline $\begin{array}{l}11 \\
12 \\
13 \\
14 \\
15\end{array}$ & $\begin{array}{l}3.9 \\
3.7 \\
3.4 \\
3.3 \\
3.2\end{array}$ & $\begin{array}{l}2.5 \\
2.5 \\
2.4 \\
2.4 \\
2.4\end{array}$ & $\begin{array}{l}2.9 \\
2.8 \\
2.8 \\
2.8 \\
2.8\end{array}$ & $\begin{array}{l}2.4 \\
2.4 \\
2.3 \\
2.3 \\
2.3\end{array}$ & $\begin{array}{l}2.1 \\
2.5 \\
2.1 \\
2.0 \\
2.0\end{array}$ & $\begin{array}{l}1.7 \\
1.7 \\
1.7 \\
1.7 \\
1.7\end{array}$ & $\begin{array}{l}1.6 \\
1.6 \\
6.0 \\
4.0 \\
3.0\end{array}$ & $\begin{array}{l}2.1 \\
2.0 \\
2.3 \\
2.1 \\
2.0\end{array}$ & $\begin{array}{r}1.9 \\
2.2 \\
5.5 \\
7.9 \\
17\end{array}$ & $\begin{array}{l}2.5 \\
2.9 \\
3.7 \\
2.7 \\
2.4\end{array}$ & $\begin{array}{l}9.3 \\
8.0 \\
5.8 \\
6.9 \\
6.6\end{array}$ & $\begin{array}{r}4.6 \\
4.5 \\
4.8 \\
5.9 \\
4.6\end{array}$ \\
\hline $\begin{array}{l}16 \\
17 \\
18 \\
19 \\
20\end{array}$ & $\begin{array}{l}3.2 \\
3.2 \\
3.2 \\
3.1 \\
3.0\end{array}$ & $\begin{array}{l}2.4 \\
2.4 \\
2.4 \\
2.4 \\
2.4\end{array}$ & $\begin{array}{l}2.7 \\
2.8 \\
2.8 \\
2.6 \\
2.7\end{array}$ & $\begin{array}{l}2.3 \\
2.3 \\
2.3 \\
2.6 \\
2.3\end{array}$ & $\begin{array}{l}2.0 \\
1.9 \\
1.9 \\
1.9 \\
1.9\end{array}$ & $\begin{array}{l}1.7 \\
2.4 \\
2.3 \\
1.8 \\
1.7\end{array}$ & $\begin{array}{l}2.3 \\
2.1 \\
2.0 \\
1.9 \\
1.9\end{array}$ & $\begin{array}{l}2.0 \\
1.9 \\
1.8 \\
1.8 \\
3.1\end{array}$ & $\begin{array}{l}6.7 \\
4.2 \\
3.4 \\
3.1 \\
2.9\end{array}$ & $\begin{array}{l}3.9 \\
5.6 \\
3.2 \\
2.7 \\
2.6\end{array}$ & $\begin{array}{l}5.3 \\
4.6 \\
4.7 \\
5.3 \\
4.9\end{array}$ & $\begin{array}{c}4.2 \\
12 \\
9.8 \\
7.3 \\
31\end{array}$ \\
\hline $\begin{array}{l}21 \\
22 \\
23 \\
24 \\
25\end{array}$ & $\begin{array}{l}3.4 \\
3.6 \\
3.4 \\
3.3 \\
2.9\end{array}$ & $\begin{array}{r}2.4 \\
6.4 \\
17 \\
7.3 \\
4.3\end{array}$ & $\begin{array}{l}2.6 \\
2.6 \\
2.5 \\
2.5 \\
2.5\end{array}$ & $\begin{array}{l}2.2 \\
2.3 \\
2.6 \\
2.7 \\
2.3\end{array}$ & $\begin{array}{l}1.9 \\
1.9 \\
1.9 \\
1.9 \\
1.9\end{array}$ & $\begin{array}{l}2.1 \\
5.5 \\
2.4 \\
2.0 \\
1.9\end{array}$ & $\begin{array}{l}1.9 \\
1.9 \\
1.9 \\
1.9 \\
1.8\end{array}$ & $\begin{array}{l}5.4 \\
2.5 \\
6.1 \\
9.0 \\
3.2\end{array}$ & $\begin{array}{l}2.9 \\
2.8 \\
2.6 \\
2.5 \\
2.5\end{array}$ & $\begin{array}{l}2.5 \\
2.5 \\
2.5 \\
3.7 \\
7.1\end{array}$ & $\begin{array}{c}6.2 \\
8.8 \\
10 \\
5.3 \\
4.6\end{array}$ & $\begin{array}{c}51 \\
16 \\
9.0 \\
6.6 \\
5.8\end{array}$ \\
\hline $\begin{array}{l}26 \\
27 \\
28 \\
29 \\
30 \\
31\end{array}$ & $\begin{array}{l}3.4 \\
3.5 \\
3.0 \\
2.9 \\
2.9 \\
2.8\end{array}$ & $\begin{array}{l}3.8 \\
3.7 \\
3.6 \\
3.5 \\
3.3 \\
-.-\end{array}$ & $\begin{array}{l}2.5 \\
2.5 \\
2.6 \\
2.4 \\
2.4 \\
2.4\end{array}$ & $\begin{array}{l}2.2 \\
2.2 \\
2.2 \\
2.2 \\
2.2 \\
2.2\end{array}$ & $\begin{array}{l}1.9 \\
1.9 \\
1.9 \\
--- \\
---\end{array}$ & $\begin{array}{l}1.8 \\
1.7 \\
1.7 \\
1.7 \\
1.7 \\
1.7\end{array}$ & $\begin{array}{l}1.9 \\
1.8 \\
1.7 \\
1.8 \\
2.6 \\
-.-\end{array}$ & $\begin{array}{l}2.5 \\
2.4 \\
2.3 \\
2.2 \\
2.2 \\
2.1\end{array}$ & $\begin{array}{l}2.5 \\
2.5 \\
3.2 \\
4.0 \\
3.2 \\
-.-\end{array}$ & $\begin{array}{l}4.9 \\
3.4 \\
3.0 \\
2.9 \\
2.8 \\
3.0\end{array}$ & $\begin{array}{r}4.3 \\
7.2 \\
7.2 \\
5.4 \\
4.9\end{array}$ & $\begin{array}{r}5.3 \\
4.9 \\
12 \\
7.8 \\
6.6\end{array}$ \\
\hline
\end{tabular}


APPENDIX III--Daily discharge--Continued

[Mean values in cubic feet per second]

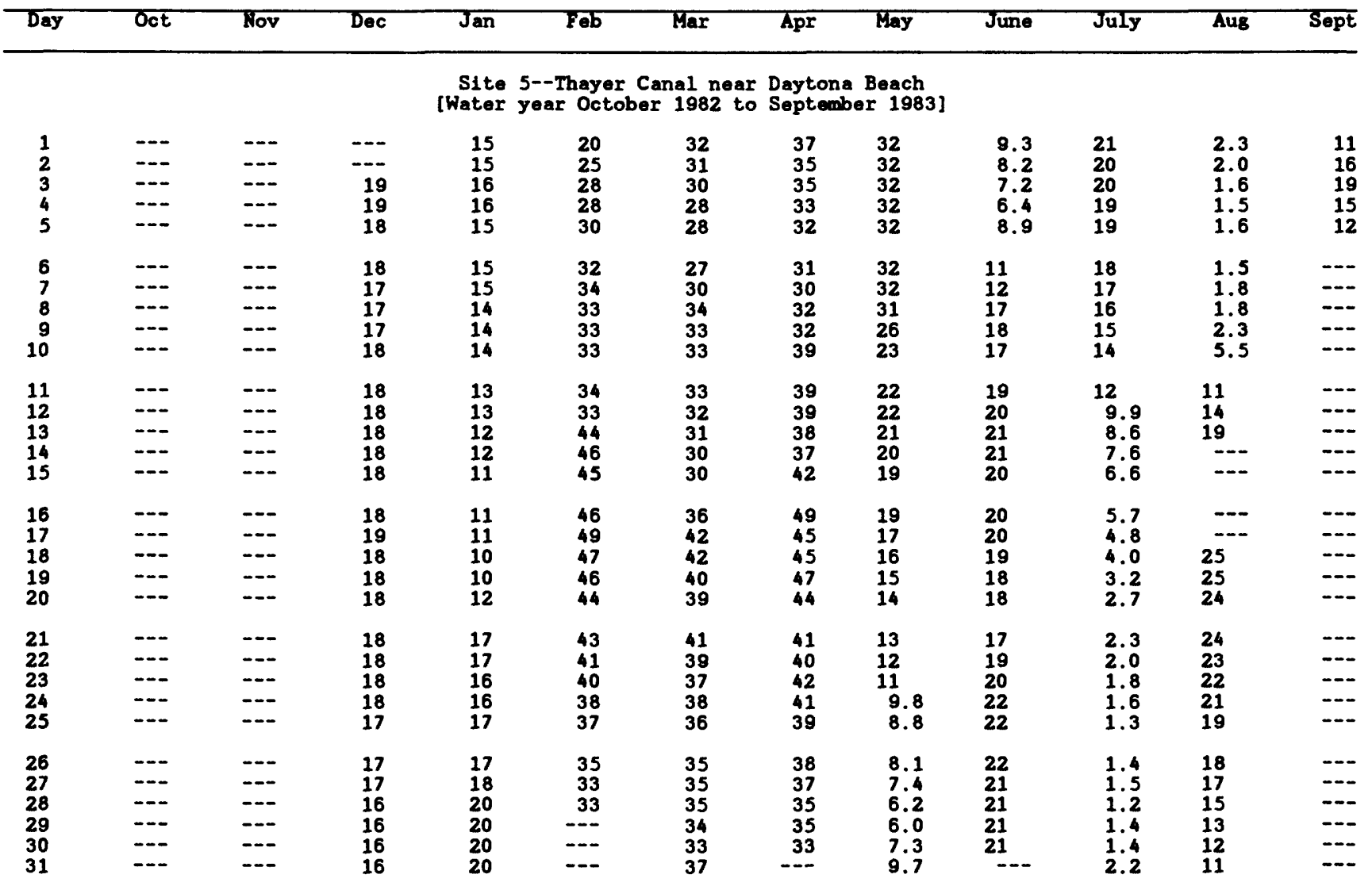

Site 5--Thayer Canal near Daytona Beach

[Water year October 1983 to September 1984]

\begin{tabular}{|c|c|c|c|c|c|c|c|c|c|c|c|c|}
\hline $\begin{array}{l}1 \\
2 \\
3 \\
4 \\
5\end{array}$ & $\overline{---}$ & $\begin{array}{l}29 \\
28 \\
27 \\
26 \\
26\end{array}$ & $\begin{array}{l}22 \\
21 \\
21 \\
21 \\
20\end{array}$ & $\begin{array}{l}52 \\
50 \\
48 \\
45 \\
43\end{array}$ & $\begin{array}{l}26 \\
25 \\
25 \\
24 \\
24\end{array}$ & $\begin{array}{l}34 \\
33 \\
33 \\
32 \\
31\end{array}$ & $\begin{array}{l}15 \\
15 \\
15 \\
41 \\
42\end{array}$ & $\begin{array}{l}18 \\
16 \\
15 \\
14 \\
16\end{array}$ & $\begin{array}{l}24 \\
23 \\
22 \\
21 \\
19\end{array}$ & $\begin{array}{l}7.5 \\
10 \\
14 \\
18 \\
20\end{array}$ & $\begin{array}{l}24 \\
26 \\
27 \\
27 \\
27\end{array}$ & $\begin{array}{l}26 \\
25 \\
23 \\
22 \\
29\end{array}$ \\
\hline $\begin{array}{r}6 \\
7 \\
8 \\
9 \\
10\end{array}$ & $\begin{array}{l}12 \\
12 \\
12 \\
14 \\
15\end{array}$ & $\begin{array}{l}26 \\
25 \\
25 \\
24 \\
25\end{array}$ & $\begin{array}{l}20 \\
20 \\
19 \\
18 \\
18\end{array}$ & $\begin{array}{l}41 \\
39 \\
37 \\
36 \\
35\end{array}$ & $\begin{array}{l}23 \\
22 \\
22 \\
21 \\
21\end{array}$ & $\begin{array}{l}30 \\
29 \\
28 \\
27 \\
25\end{array}$ & $\begin{array}{l}37 \\
35 \\
33 \\
32 \\
32\end{array}$ & $\begin{array}{l}17 \\
16 \\
14 \\
12 \\
11\end{array}$ & $\begin{array}{l}17 \\
15 \\
14 \\
12 \\
11\end{array}$ & $\begin{array}{l}26 \\
30 \\
32 \\
32 \\
32\end{array}$ & $\begin{array}{l}26 \\
25 \\
25 \\
23 \\
22\end{array}$ & $\begin{array}{l}40 \\
53 \\
47 \\
61 \\
79\end{array}$ \\
\hline $\begin{array}{l}11 \\
12 \\
13 \\
14 \\
15\end{array}$ & $\begin{array}{l}15 \\
15 \\
13 \\
12 \\
13\end{array}$ & $\begin{array}{l}25 \\
26 \\
27 \\
27 \\
26\end{array}$ & $\begin{array}{l}17 \\
39 \\
39 \\
40 \\
42\end{array}$ & $\begin{array}{l}35 \\
33 \\
33 \\
32 \\
31\end{array}$ & $\begin{array}{l}20 \\
20 \\
23 \\
28 \\
28\end{array}$ & $\begin{array}{l}25 \\
24 \\
23 \\
23 \\
22\end{array}$ & $\begin{array}{l}30 \\
28 \\
28 \\
27 \\
28\end{array}$ & $\begin{array}{l}9.6 \\
8.5 \\
7.5 \\
6.6 \\
5.9\end{array}$ & $\begin{array}{l}9.3 \\
8.3 \\
7.6 \\
7.2 \\
6.3\end{array}$ & $\begin{array}{l}32 \\
32 \\
32 \\
32 \\
30\end{array}$ & $\begin{array}{l}21 \\
19 \\
19 \\
18 \\
18\end{array}$ & $\begin{array}{l}63 \\
56 \\
51 \\
47 \\
45\end{array}$ \\
\hline $\begin{array}{l}16 \\
17 \\
18 \\
19 \\
20\end{array}$ & $\begin{array}{l}26 \\
43 \\
42 \\
37 \\
35\end{array}$ & $\begin{array}{l}26 \\
24 \\
23 \\
23 \\
23\end{array}$ & $\begin{array}{l}42 \\
41 \\
45 \\
47 \\
56\end{array}$ & $\begin{array}{l}30 \\
30 \\
29 \\
30 \\
30\end{array}$ & $\begin{array}{l}28 \\
29 \\
28 \\
28 \\
27\end{array}$ & $\begin{array}{l}21 \\
21 \\
20 \\
20 \\
19\end{array}$ & $\begin{array}{l}27 \\
26 \\
27 \\
26 \\
26\end{array}$ & $\begin{array}{l}5.2 \\
4.6 \\
4.1 \\
3.7 \\
3.3\end{array}$ & $\begin{array}{l}5.5 \\
4.8 \\
4.3 \\
3.8 \\
3.4\end{array}$ & $\begin{array}{l}29 \\
28 \\
26 \\
26 \\
28\end{array}$ & $\begin{array}{l}18 \\
19 \\
19 \\
19 \\
20\end{array}$ & $\begin{array}{l}42 \\
41 \\
45 \\
49 \\
45\end{array}$ \\
\hline $\begin{array}{l}21 \\
22 \\
23 \\
24 \\
25\end{array}$ & $\begin{array}{l}33 \\
39 \\
39 \\
37 \\
37\end{array}$ & $\begin{array}{l}24 \\
23 \\
23 \\
23 \\
23\end{array}$ & $\begin{array}{l}74 \\
64 \\
58 \\
54 \\
50\end{array}$ & $\begin{array}{l}31 \\
31 \\
31 \\
31 \\
31\end{array}$ & $\begin{array}{l}27 \\
33 \\
37 \\
37 \\
37\end{array}$ & $\begin{array}{l}20 \\
19 \\
18 \\
18 \\
17\end{array}$ & $\begin{array}{l}25 \\
25 \\
24 \\
24 \\
23\end{array}$ & $\begin{array}{l}3.0 \\
2.7 \\
11 \\
19 \\
20\end{array}$ & $\begin{array}{l}3.0 \\
2.7 \\
2.7 \\
2.8 \\
3.1\end{array}$ & $\begin{array}{l}30 \\
30 \\
32 \\
29 \\
27\end{array}$ & $\begin{array}{l}22 \\
24 \\
26 \\
27 \\
31\end{array}$ & $\begin{array}{l}43 \\
41 \\
40 \\
39 \\
37\end{array}$ \\
\hline $\begin{array}{l}26 \\
27 \\
28 \\
29 \\
30 \\
31\end{array}$ & $\begin{array}{l}36 \\
35 \\
33 \\
32 \\
31 \\
30\end{array}$ & $\begin{array}{l}22 \\
22 \\
22 \\
22 \\
22 \\
-\end{array}$ & $\begin{array}{l}47 \\
44 \\
43 \\
54 \\
64 \\
55\end{array}$ & $\begin{array}{l}31 \\
31 \\
30 \\
29 \\
28 \\
27\end{array}$ & $\begin{array}{r}36 \\
36 \\
37 \\
35 \\
--- \\
--\end{array}$ & $\begin{array}{l}17 \\
17 \\
18 \\
18 \\
16 \\
16\end{array}$ & $\begin{array}{r}22 \\
22 \\
21 \\
20 \\
19 \\
-\end{array}$ & $\begin{array}{l}21 \\
26 \\
27 \\
26 \\
26 \\
25\end{array}$ & $\begin{array}{l}4.5 \\
4.2 \\
4.2 \\
5.0 \\
6.3 \\
\end{array}$ & $\begin{array}{l}26 \\
25 \\
24 \\
24 \\
23 \\
24\end{array}$ & $\begin{array}{l}35 \\
34 \\
32 \\
31 \\
29 \\
28\end{array}$ & $\begin{array}{r}36 \\
45 \\
90 \\
70 \\
62 \\
-\end{array}$ \\
\hline
\end{tabular}


APPENDIX III--Daily discharge--Continued

[Mean values in cubic feet per second]

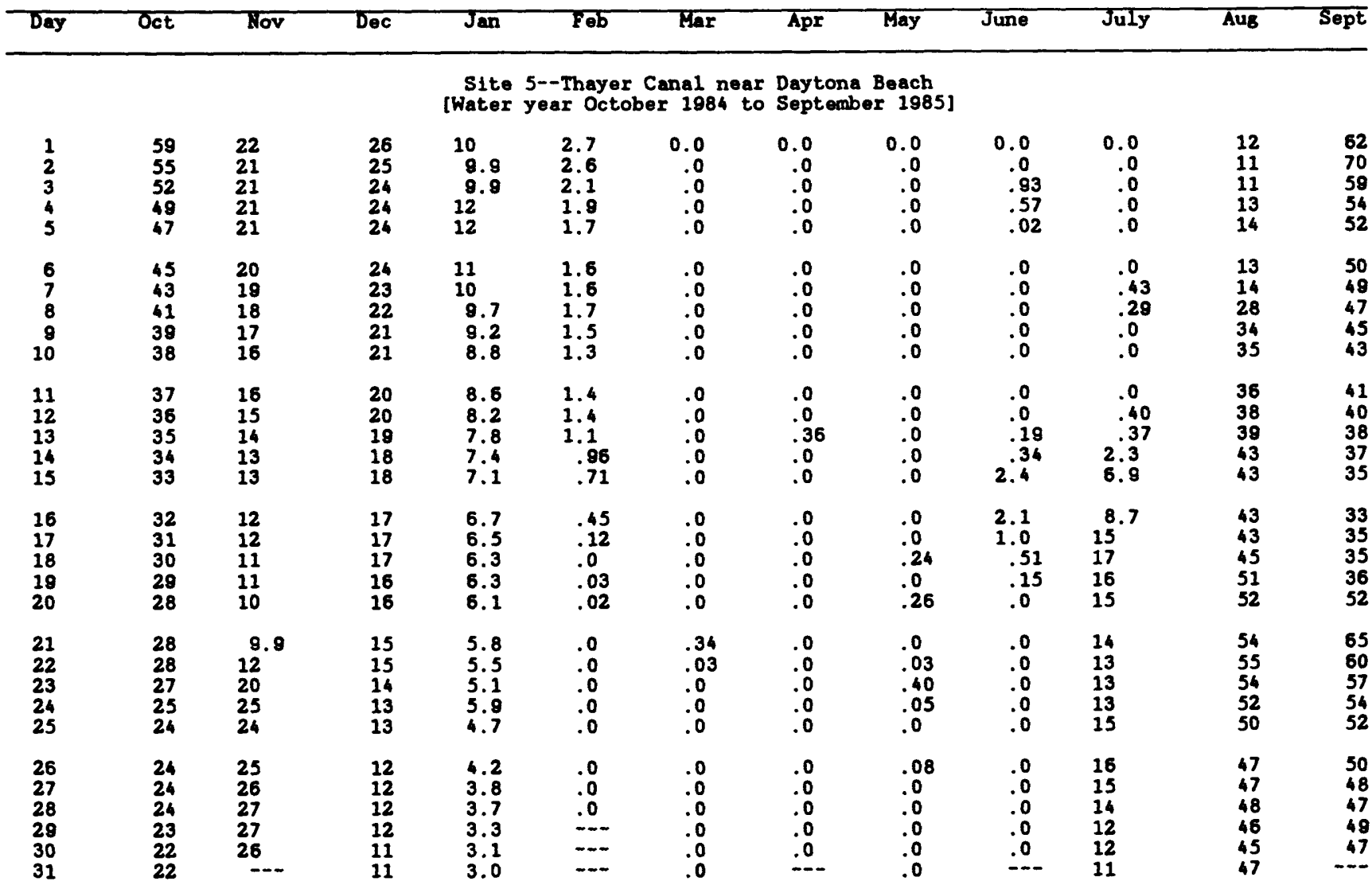

Site 6--Bayless Boulevard Canal at Daytona Beach [Water year October 1982 to September 1983]

\begin{tabular}{|c|c|c|c|c|c|c|c|c|c|c|c|c|}
\hline $\begin{array}{l}1 \\
2 \\
3 \\
4 \\
5\end{array}$ & $\begin{array}{l}-\infty \\
-- \\
-- \\
-\infty \\
--\end{array}$ & $\begin{array}{l}m- \\
--- \\
\cdots- \\
\cdots-\end{array}$ & $\begin{array}{l}-\infty \\
-\infty \\
-\infty \\
-\infty\end{array}$ & $\begin{array}{r}0.53 \\
.62 \\
.75 \\
.59 \\
.59\end{array}$ & $\begin{array}{r}1.8 \\
12 \\
3.6 \\
2.5 \\
1.7\end{array}$ & $\begin{array}{l}1.5 \\
1.6 \\
1.3 \\
1.2 \\
1.2\end{array}$ & $\begin{array}{l}3.2 \\
2.7 \\
2.5 \\
2.1 \\
1.9\end{array}$ & $\begin{array}{l}1.5 \\
1.5 \\
1.5 \\
1.6 \\
1.7\end{array}$ & $\begin{array}{c}1.3 \\
.90 \\
.80 \\
.85 \\
2.5\end{array}$ & $\begin{array}{l}-- \\
-- \\
-- \\
--\end{array}$ & $\begin{array}{l}--\infty \\
--- \\
-\infty \\
---\end{array}$ & $\begin{array}{l}1.8 \\
2.7 \\
2.8 \\
1.5 \\
.88\end{array}$ \\
\hline $\begin{array}{r}6 \\
7 \\
8 \\
9 \\
10\end{array}$ & $\begin{array}{l}-- \\
-- \\
-- \\
--\end{array}$ & $m$ & $\begin{array}{r}.66 \\
.81 \\
1.1 \\
.80 \\
1.9\end{array}$ & $\begin{array}{r}.56 \\
.51 \\
.57 \\
.58 \\
.59\end{array}$ & $\begin{array}{l}2.9 \\
2.5 \\
1.9 \\
1.6 \\
2.0\end{array}$ & $\begin{array}{l}1.2 \\
5.5 \\
3.4 \\
2.5 \\
2.1\end{array}$ & $\begin{array}{l}1.8 \\
3.3 \\
4.9 \\
3.7 \\
6.2\end{array}$ & $\begin{array}{l}1.5 \\
1.3 \\
1.2 \\
1.2 \\
1.1\end{array}$ & $\begin{array}{l}2.4 \\
14 \\
16 \\
3.3 \\
2.1\end{array}$ & $\begin{array}{l}m- \\
-\infty \\
m- \\
-\infty\end{array}$ & $\frac{--}{--}$ & $\begin{array}{r}.69 \\
.59 \\
.51 \\
.47 \\
.42\end{array}$ \\
\hline $\begin{array}{l}11 \\
12 \\
13 \\
14 \\
15\end{array}$ & $\begin{array}{l}-\infty \\
--- \\
--- \\
-- \\
---\end{array}$ & $\begin{array}{l}-\cdots \\
-\cdots \\
-\cdots\end{array}$ & $\begin{array}{r}1.3 \\
1.1 \\
.94 \\
.80 \\
.75\end{array}$ & $\begin{array}{r}.56 \\
.51 \\
.44 \\
.54 \\
.57\end{array}$ & $\begin{array}{r}2.9 \\
3.7 \\
21 \\
5.1 \\
3.3\end{array}$ & $\begin{array}{l}1.7 \\
1.5 \\
1.5 \\
1.4 \\
5.6\end{array}$ & $\begin{array}{l}3.4 \\
2.9 \\
2.5 \\
2.2 \\
7.5\end{array}$ & $\begin{array}{r}1.2 \\
1.1 \\
1.1 \\
.98 \\
.97\end{array}$ & $\begin{array}{l}1.6 \\
1.2 \\
7.6 \\
1.9 \\
1.4\end{array}$ & $\begin{array}{l}m \\
m- \\
m- \\
m-\end{array}$ & $\begin{array}{l}1.6 \\
1.7 \\
6.6 \\
7.4 \\
4.5\end{array}$ & $\begin{array}{l}.37 \\
.29 \\
.29 \\
.29 \\
.29\end{array}$ \\
\hline $\begin{array}{l}16 \\
17 \\
18 \\
18 \\
20\end{array}$ & $\begin{array}{l}--- \\
--- \\
--- \\
-- \\
--\end{array}$ & 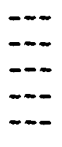 & $\begin{array}{l}.81 \\
.80 \\
.73 \\
.70 \\
.66\end{array}$ & $\begin{array}{r}.51 \\
.45 \\
.44 \\
.44 \\
2.1\end{array}$ & $\begin{array}{l}3.4 \\
3.3 \\
2.6 \\
2.3 \\
2.0\end{array}$ & $\begin{array}{l}12 \\
16 \\
5.0 \\
3.4 \\
2.9\end{array}$ & $\begin{array}{l}4.9 \\
3.1 \\
2.9 \\
4.6 \\
3.0\end{array}$ & $\begin{array}{l}.92 \\
.84 \\
.79 \\
.79 \\
.79\end{array}$ & $\begin{array}{l}1.1 \\
1.1 \\
1.0 \\
.81 \\
.67\end{array}$ & $\begin{array}{l}-- \\
-- \\
-- \\
--\end{array}$ & $\begin{array}{l}2.6 \\
1.7 \\
1.3 \\
.98 \\
.80\end{array}$ & $\begin{array}{l}.22 \\
3.1 \\
1.7 \\
1.2 \\
2.3\end{array}$ \\
\hline $\begin{array}{l}21 \\
22 \\
23 \\
24 \\
25\end{array}$ & $\begin{array}{l}-- \\
-- \\
-- \\
-- \\
--\end{array}$ & $\overline{-}$ & $\begin{array}{l}.65 \\
.64 \\
.59 \\
.59 \\
.58\end{array}$ & $\begin{array}{l}2.0 \\
1.0 \\
1.0 \\
1.8 \\
1.7\end{array}$ & $\begin{array}{l}1.8 \\
1.8 \\
1.7 \\
1.6 \\
1.5\end{array}$ & $\begin{array}{l}3.7 \\
2.7 \\
2.3 \\
2.9 \\
2.3\end{array}$ & $\begin{array}{l}2.5 \\
2.2 \\
3.8 \\
4.1 \\
2.8\end{array}$ & $\begin{array}{l}.78 \\
.76 \\
.72 \\
.69 \\
.70\end{array}$ & $\begin{array}{l}3.2 \\
3.3 \\
2.4 \\
3.3 \\
2.6\end{array}$ & $\begin{array}{l}m- \\
m- \\
-\cdots \\
-- \\
m-\end{array}$ & $\begin{array}{r}.67 \\
.54 \\
.47 \\
.46 \\
.51\end{array}$ & $\begin{array}{r}1.2 \\
.91 \\
.72 \\
.86 \\
6.6\end{array}$ \\
\hline $\begin{array}{l}26 \\
27 \\
28 \\
29 \\
30 \\
31\end{array}$ & $\begin{array}{l}--- \\
--- \\
--- \\
--- \\
---\end{array}$ & $\begin{array}{l}--- \\
-\ldots \\
-\cdots \\
-- \\
--\end{array}$ & $\begin{array}{l}.58 \\
.58 \\
.53 \\
.51 \\
.51 \\
.51\end{array}$ & $\begin{array}{l}1.3 \\
1.5 \\
1.6 \\
1.1 \\
1.0 \\
1.3\end{array}$ & $\begin{array}{l}1.4 \\
1.3 \\
1.4 \\
\ldots- \\
-\ldots\end{array}$ & $\begin{array}{l}2.0 \\
2.5 \\
2.8 \\
2.1 \\
1.9 \\
5.2\end{array}$ & $\begin{array}{l}2.4 \\
2.1 \\
1.9 \\
1.7 \\
1.6 \\
---\end{array}$ & \begin{tabular}{c}
.84 \\
.79 \\
\hdashline- \\
2.1
\end{tabular} & $\begin{array}{r}1.6 \\
.96 \\
.72 \\
.65 \\
-.-\end{array}$ & 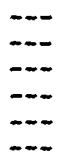 & $\begin{array}{l}.57 \\
.42 \\
.38 \\
.41 \\
.33 \\
.29\end{array}$ & $\begin{array}{r}1.6 \\
1.2 \\
.90 \\
.73 \\
.62 \\
-\end{array}$ \\
\hline
\end{tabular}


APPENDIX III--Daily discharge--Continued

[Mean values in cubic feet per second]

\begin{tabular}{|c|c|c|c|c|c|c|c|c|c|c|c|c|}
\hline Day & Oct & Kov & Dec & $\operatorname{Jan}$ & Fकb & Mar & Apr & May & June & July & Aus & Sept \\
\hline \multicolumn{13}{|c|}{$\begin{array}{l}\text { Site 6--Bayless Boulevard Canal at Daytona Beach } \\
\text { [Water year October } 1983 \text { to September 1984] }\end{array}$} \\
\hline $\begin{array}{l}1 \\
2 \\
3 \\
4 \\
5\end{array}$ & $\begin{array}{r}0.53 \\
.44 \\
.42 \\
.37 \\
.37\end{array}$ & $\begin{array}{r}0.75 \\
.73 \\
.68 \\
.74 \\
.86\end{array}$ & $\begin{array}{r}0.44 \\
.41 \\
.37 \\
.37 \\
.37\end{array}$ & $\begin{array}{l}2.1 \\
1.7 \\
1.5 \\
1.3 \\
1.2\end{array}$ & $\begin{array}{r}0.59 \\
.59 \\
.59 \\
.59 \\
.52\end{array}$ & $\begin{array}{r}0.95 \\
.69 \\
.66 \\
.66 \\
.67\end{array}$ & $\begin{array}{r}0.51 \\
.51 \\
4.3 \\
21 \\
2.9\end{array}$ & $\begin{array}{l}--- \\
--- \\
--- \\
---\end{array}$ & $\begin{array}{r}0.61 \\
.52 \\
.49 \\
.44 \\
.44\end{array}$ & $\begin{array}{l}7.4 \\
7.6 \\
6.8 \\
4.4 \\
2.4\end{array}$ & $\begin{array}{r}0.75 \\
.68 \\
.42 \\
.35 \\
.35\end{array}$ & $\begin{array}{r}0.26 \\
.20 \\
.17 \\
.13 \\
8.4\end{array}$ \\
\hline $\begin{array}{r}6 \\
7 \\
8 \\
9 \\
10\end{array}$ & $\begin{array}{r}.37 \\
.36 \\
.35 \\
1.1 \\
.78\end{array}$ & $\begin{array}{l}.73 \\
.66 \\
.66 \\
.62 \\
.63\end{array}$ & $\begin{array}{l}.37 \\
.37 \\
.37 \\
.37 \\
.37\end{array}$ & $\begin{array}{c}1.1 \\
.95 \\
.85 \\
.80 \\
1.1\end{array}$ & $\begin{array}{l}.51 \\
.51 \\
.48 \\
.44 \\
.44\end{array}$ & $\begin{array}{l}.63 \\
.59 \\
.59 \\
.59 \\
.59\end{array}$ & $\begin{array}{c}1.7 \\
1.2 \\
.99 \\
1.0 \\
.87\end{array}$ & $\begin{array}{l}-.- \\
-.- \\
-.- \\
.44 \\
.40\end{array}$ & $\begin{array}{l}.44 \\
.44 \\
.44 \\
.44 \\
.44\end{array}$ & $\begin{array}{r}1.6 \\
1.3 \\
1.1 \\
.79 \\
.68\end{array}$ & $\begin{array}{l}.24 \\
.23 \\
.20 \\
.15 \\
.12\end{array}$ & $\begin{array}{c}14 \\
21 \\
3.9 \\
22 \\
27\end{array}$ \\
\hline $\begin{array}{l}11 \\
12 \\
13 \\
14 \\
15\end{array}$ & $\begin{array}{c}1.8 \\
1.5 \\
.94 \\
.74 \\
1.7\end{array}$ & $\begin{array}{l}.59 \\
.59 \\
.54 \\
.51 \\
.51\end{array}$ & $\begin{array}{l}40.76 \\
5.4 \\
2.7 \\
2.6\end{array}$ & $\begin{array}{r}1.1 \\
.89 \\
.84 \\
.76 \\
.73\end{array}$ & $\begin{array}{l}.44 \\
.47 \\
5.1 \\
2.5 \\
1.2\end{array}$ & $\begin{array}{l}.59 \\
.59 \\
.59 \\
.59 \\
.59\end{array}$ & $\begin{array}{r}.66 \\
.61 \\
.59 \\
1.7 \\
2.9\end{array}$ & $\begin{array}{l}.37 \\
.37 \\
.37 \\
.37 \\
.37\end{array}$ & $\begin{array}{l}.44 \\
.44 \\
.44 \\
.44 \\
.44\end{array}$ & $\begin{array}{l}.66 \\
.60 \\
.67 \\
.65 \\
1.1\end{array}$ & $\begin{array}{l}.09 \\
1.4 \\
1.2 \\
.30 \\
.15\end{array}$ & $\begin{array}{l}4.9 \\
2.5 \\
1.7 \\
1.3 \\
1.2\end{array}$ \\
\hline $\begin{array}{l}16 \\
17 \\
18 \\
19 \\
20\end{array}$ & $\begin{array}{l}39 \\
31 \\
8.4 \\
3.0 \\
2.1\end{array}$ & $\begin{array}{l}.61 \\
.51 \\
.46 \\
.44 \\
.92\end{array}$ & $\begin{array}{l}2.1 \\
1.7 \\
2.7 \\
2.0 \\
25\end{array}$ & $\begin{array}{l}.72 \\
.66 \\
.69 \\
1.5 \\
1.4\end{array}$ & $\begin{array}{l}.86 \\
.68 \\
.60 \\
.59 \\
.59\end{array}$ & $\begin{array}{l}.59 \\
.59 \\
.59 \\
.59 \\
.64\end{array}$ & $\begin{array}{l}1.0 \\
.75 \\
.73 \\
.66 \\
.66\end{array}$ & $\begin{array}{l}.37 \\
.37 \\
.37 \\
.37 \\
.37\end{array}$ & $\begin{array}{l}.44 \\
.44 \\
.44 \\
.44 \\
.42\end{array}$ & $\begin{array}{l}.57 \\
1.1 \\
1.2 \\
1.5 \\
1.8\end{array}$ & $\begin{array}{l}.08 \\
.06 \\
.06 \\
.41 \\
.78\end{array}$ & $\begin{array}{l}-- \\
--- \\
--- \\
--- \\
--\end{array}$ \\
\hline $\begin{array}{l}21 \\
22 \\
23 \\
24 \\
25\end{array}$ & $\begin{array}{l}1.7 \\
5.9 \\
2.8 \\
2.0 \\
1.6\end{array}$ & $\begin{array}{l}.86 \\
.48 \\
.44 \\
.44 \\
.45\end{array}$ & $\begin{array}{r}24 \\
5.0 \\
2.9 \\
2.3 \\
1.8\end{array}$ & $\begin{array}{r}2.8 \\
1.7 \\
1.3 \\
1.0 \\
.93\end{array}$ & $\begin{array}{l}.62 \\
9.6 \\
3.0 \\
1.6 \\
1.1\end{array}$ & $\begin{array}{r}1.1 \\
.59 \\
.59 \\
.54 \\
.51\end{array}$ & $\begin{array}{l}.66 \\
.61 \\
.60 \\
.65 \\
.59\end{array}$ & $\begin{array}{r}.32 \\
24^{.29} \\
4.6 \\
2.3\end{array}$ & $\begin{array}{l}.39 \\
.37 \\
.49 \\
.45 \\
.47\end{array}$ & $\begin{array}{l}6.0 \\
3.1 \\
3.8 \\
2.3 \\
1.3\end{array}$ & $\begin{array}{r}1.9 \\
1.2 \\
.50 \\
.34 \\
3.9\end{array}$ & $\begin{array}{l}--- \\
--- \\
--- \\
--- \\
---\end{array}$ \\
\hline $\begin{array}{l}26 \\
27 \\
28 \\
29 \\
30 \\
31\end{array}$ & $\begin{array}{l}1.3 \\
1.1 \\
.95 \\
.86 \\
.80 \\
.75\end{array}$ & $\begin{array}{l}.41 \\
.37 \\
.37 \\
.95 \\
.63 \\
-.--\end{array}$ & $\begin{array}{r}1.5 \\
1.5 \\
1.5 \\
19 \\
7.6 \\
2.8\end{array}$ & $\begin{array}{l}.87 \\
.85 \\
.71 \\
.66 \\
.62 \\
.59\end{array}$ & $\begin{array}{l}.82 \\
1.2 \\
2.0 \\
1.3 \\
--- \\
---\end{array}$ & $\begin{array}{r}.51 \\
.59 \\
.54 \\
.51 \\
.51 \\
.51\end{array}$ & $\begin{array}{l}.59 \\
.59 \\
.59 \\
.59 \\
---- \\
---\end{array}$ & $\begin{array}{l}2.6 \\
2.8 \\
1.9 \\
1.2 \\
1.0 \\
.81\end{array}$ & $\begin{array}{l}.79 \\
.37 \\
.45 \\
1.5 \\
2.2 \\
-.-\end{array}$ & $\begin{array}{l}.89 \\
.69 \\
.57 \\
.48 \\
.43 \\
.67\end{array}$ & $\begin{array}{r}3.0 \\
1.1 \\
.74 \\
.56 \\
.45 \\
.33\end{array}$ & $\begin{array}{l}--- \\
--- \\
--- \\
-- \\
--\end{array}$ \\
\hline
\end{tabular}

Site 6--Bayless Boulevard Canal at Daytona Beach [Water year October 1984 to September 1985]

\begin{tabular}{|c|c|c|c|c|c|c|c|c|c|c|c|c|}
\hline $\begin{array}{l}1 \\
2 \\
3 \\
4 \\
5\end{array}$ & $\begin{array}{l}--- \\
--- \\
--- \\
--- \\
---\end{array}$ & $\begin{array}{r}0.0 \\
.0 \\
.0 \\
.0 \\
.0\end{array}$ & $\begin{array}{r}0.13 \\
.11 \\
.08 \\
.07 \\
.09\end{array}$ & $\begin{array}{r}0.0 \\
.0 \\
2.3 \\
.50 \\
.0\end{array}$ & $\begin{array}{r}0.0 \\
.0 \\
.0 \\
.0 \\
.0\end{array}$ & $\begin{array}{r}0.0 \\
.0 \\
.0 \\
.0 \\
.0\end{array}$ & $\begin{array}{r}0.0 \\
.0 \\
.0 \\
.0 \\
.0\end{array}$ & $\begin{array}{r}0.0 \\
.0 \\
.0 \\
3.5 \\
.34\end{array}$ & $\begin{array}{r}0.0 \\
.0 \\
.0 \\
.0 \\
.0\end{array}$ & $\begin{array}{l}0.11 \\
.0 \\
.0 \\
.0 \\
.02\end{array}$ & $\begin{array}{r}4.1 \\
2.4 \\
1.5 \\
2.7 \\
.75\end{array}$ & $\begin{array}{r}36 \\
19 \\
4.8 \\
2.5 \\
1.6\end{array}$ \\
\hline $\begin{array}{r}6 \\
7 \\
8 \\
9 \\
10\end{array}$ & $\begin{array}{l}--- \\
--- \\
--- \\
---\end{array}$ & $\begin{array}{l}.0 \\
.0 \\
.0 \\
.0 \\
.0\end{array}$ & $\begin{array}{l}.02 \\
.0 \\
.0 \\
.0 \\
.0\end{array}$ & $\begin{array}{l}.0 \\
.0 \\
.0 \\
.0 \\
.0\end{array}$ & $\begin{array}{l}.03 \\
.0 \\
.01 \\
.0 \\
.0\end{array}$ & $\begin{array}{l}.0 \\
.0 \\
.0 \\
.0 \\
.0\end{array}$ & $\begin{array}{l}.03 \\
.0 \\
.0 \\
.0 \\
.0\end{array}$ & $\begin{array}{l}.0 \\
.0 \\
.0 \\
.0 \\
.0\end{array}$ & $\begin{array}{l}.0 \\
.0 \\
.58 \\
.65 \\
.0\end{array}$ & $\begin{array}{c}.55 \\
.43 \\
1.2 \\
.01 \\
.0\end{array}$ & $\begin{array}{c}8.35 \\
19 \\
8.5 \\
3.8\end{array}$ & $\begin{array}{l}2.8 \\
2.4 \\
1.6 \\
1.0 \\
.98\end{array}$ \\
\hline $\begin{array}{l}11 \\
12 \\
13 \\
14 \\
15\end{array}$ & $\begin{array}{l}--- \\
--- \\
--- \\
---\end{array}$ & $\begin{array}{l}.0 \\
.0 \\
.0 \\
.0 \\
.0\end{array}$ & $\begin{array}{l}.0 \\
.0 \\
.0 \\
.0 \\
.0\end{array}$ & $\begin{array}{l}.0 \\
.0 \\
.0 \\
.0 \\
.0\end{array}$ & $\begin{array}{l}.0 \\
.0 \\
.0 \\
.0 \\
.0\end{array}$ & $\begin{array}{l}.0 \\
.0 \\
.0 \\
.0 \\
.0\end{array}$ & $\begin{array}{c}.0 \\
.0 \\
1.7 \\
.12 \\
.0\end{array}$ & $\begin{array}{l}.0 \\
.0 \\
.0 \\
.0 \\
.0\end{array}$ & $\begin{array}{r}.0 \\
.46 \\
.88 \\
2.5\end{array}$ & $\begin{array}{l}.0 \\
.18 \\
.30 \\
.0 \\
.0\end{array}$ & $\begin{array}{l}2.5 \\
2.7 \\
2.2 \\
2.8 \\
3.6\end{array}$ & $\begin{array}{l}.63 \\
.38 \\
.24 \\
.44 \\
.14\end{array}$ \\
\hline $\begin{array}{l}16 \\
17 \\
18 \\
19 \\
20\end{array}$ & $\begin{array}{l}--- \\
--- \\
--- \\
--- \\
---\end{array}$ & $\begin{array}{l}.0 \\
.0 \\
.0 \\
.0 \\
.0\end{array}$ & $\begin{array}{l}.0 \\
.0 \\
.0 \\
.0 \\
.0\end{array}$ & $\begin{array}{l}.0 \\
.0 \\
.0 \\
.0 \\
.0\end{array}$ & $\begin{array}{l}.0 \\
.0 \\
.0 \\
.0 \\
.0\end{array}$ & $\begin{array}{l}.0 \\
.11 \\
.01 \\
.0 \\
.0\end{array}$ & $\begin{array}{l}.0 \\
.0 \\
.0 \\
.0 \\
.0\end{array}$ & $\begin{array}{l}.0 \\
.0 \\
.0 \\
.0 \\
.48\end{array}$ & $\begin{array}{r}4.4 \\
1.5 \\
.54 \\
.24 \\
.06\end{array}$ & $\begin{array}{l}1.0 \\
.73 \\
.01 \\
.0 \\
.0\end{array}$ & $\begin{array}{l}3.0 \\
2.1 \\
1.6 \\
4.5 \\
3.2\end{array}$ & $\begin{array}{l}.04 \\
6.2 \\
2.4 \\
1.8 \\
21\end{array}$ \\
\hline $\begin{array}{l}21 \\
22 \\
23 \\
24 \\
25\end{array}$ & $\begin{array}{l}--- \\
--- \\
--- \\
---\end{array}$ & $\begin{array}{r}1.0 \\
1.5 \\
2.1 \\
.84\end{array}$ & $\begin{array}{l}.0 \\
.0 \\
.0 \\
.0 \\
.0\end{array}$ & $\begin{array}{l}.0 \\
.0 \\
.0 \\
.0 \\
.0\end{array}$ & $\begin{array}{l}.0 \\
.0 \\
.0 \\
.0 \\
.0\end{array}$ & $\begin{array}{l}.31 \\
.78 \\
.0 \\
.0 \\
.0\end{array}$ & $\begin{array}{l}.0 \\
.0 \\
.0 \\
.0 \\
.0\end{array}$ & $\begin{array}{l}.39 \\
.01 \\
1.9 \\
1.5 \\
.0\end{array}$ & $\begin{array}{l}.0 \\
.0 \\
.0 \\
.0 \\
.0\end{array}$ & $\begin{array}{l}.0 \\
.0 \\
.0 \\
.10 \\
.83\end{array}$ & $\begin{array}{l}2.8 \\
8.2 \\
4.9 \\
2.1 \\
1.4\end{array}$ & $\begin{array}{r}27 \\
6.6 \\
3.2 \\
2.0 \\
1.5\end{array}$ \\
\hline $\begin{array}{l}26 \\
27 \\
28 \\
29 \\
30 \\
31\end{array}$ & $\begin{array}{l}--- \\
--- \\
--- \\
-0 \\
0\end{array}$ & $\begin{array}{l}.61 \\
.50 \\
.44 \\
.32 \\
.20 \\
.--\end{array}$ & $\begin{array}{l}.0 \\
.0 \\
.0 \\
.0 \\
.0 \\
.0\end{array}$ & $\begin{array}{l}.0 \\
.0 \\
.0 \\
.0 \\
.0 \\
.0\end{array}$ & $\begin{array}{l}.0 \\
.0 \\
.0 \\
--- \\
---\end{array}$ & $\begin{array}{l}.0 \\
.0 \\
.0 \\
.0 \\
.0 \\
.0\end{array}$ & $\begin{array}{l}.0 \\
.0 \\
.0 \\
.0 \\
.0 \\
-0-\end{array}$ & $\begin{array}{l}.0 \\
.0 \\
.0 \\
.0 \\
.0 \\
.0\end{array}$ & $\begin{array}{l}.0 \\
.0 \\
.61 \\
.21 \\
.13 \\
.--\end{array}$ & $\begin{array}{l}.25 \\
.0 \\
.0 \\
.0 \\
.0 \\
.89\end{array}$ & $\begin{array}{l}1.1 \\
2.5 \\
3.3 \\
2.5 \\
1.4 \\
7.0\end{array}$ & $\begin{array}{l}1.1 \\
.86 \\
3.6 \\
1.5 \\
1.3 \\
---\end{array}$ \\
\hline
\end{tabular}


APPENDIX III--Daily discharge--Continued

[Mean values in cubic feet per second]

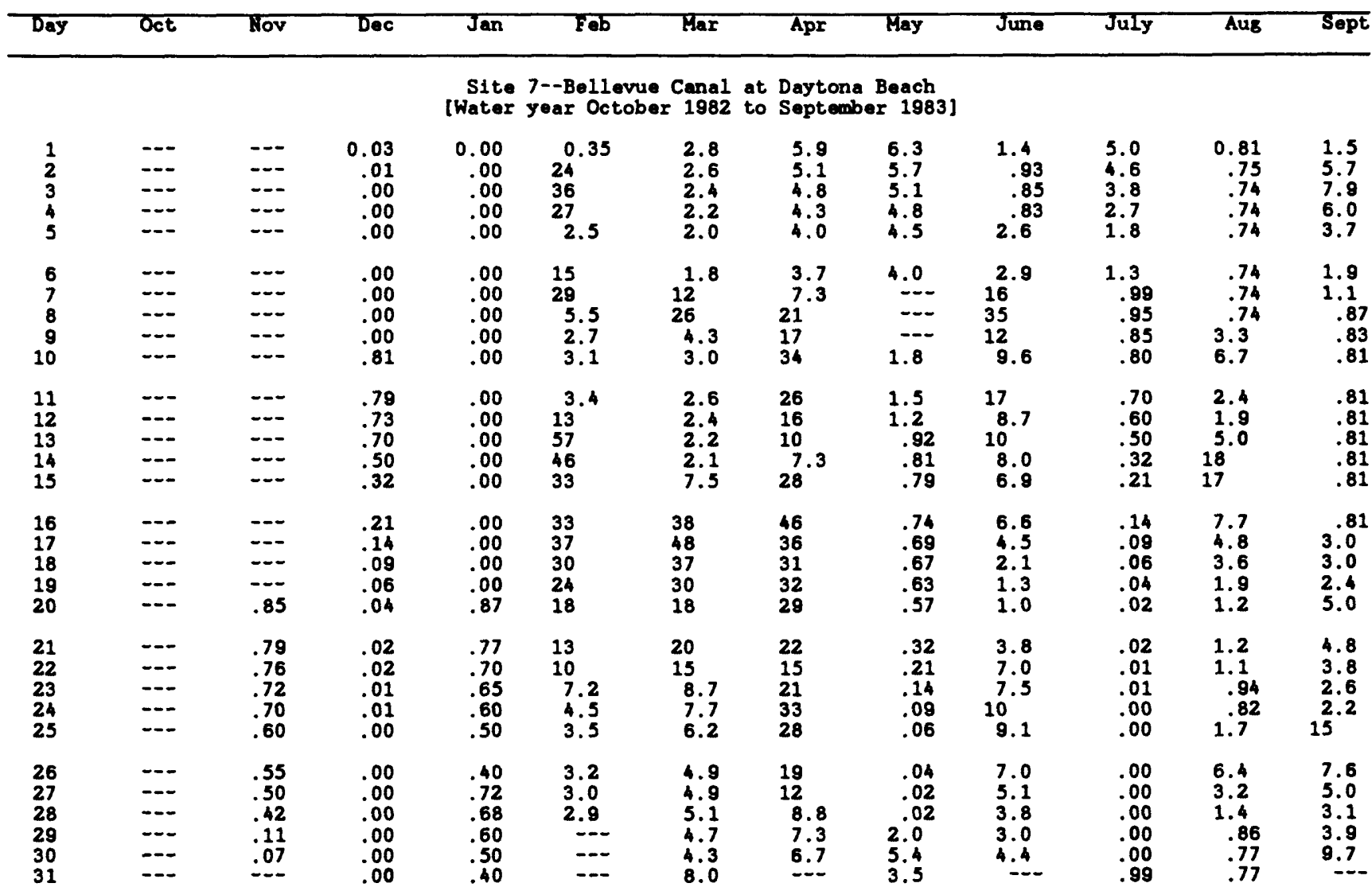

Site 7--Bellevre Canal at Daytona Beach

[Water year October 1983 to September 1984]

\begin{tabular}{|c|c|c|c|c|c|c|c|c|c|c|c|}
\hline $\begin{array}{r}4.5 \\
1.8 \\
1.1 \\
.84 \\
.81\end{array}$ & $\begin{array}{l}5.0 \\
4.7 \\
4.3 \\
3.8 \\
3.4\end{array}$ & $\begin{array}{c}1.6 \\
1.7 \\
1.4 \\
1.0 \\
.87\end{array}$ & $\begin{array}{l}36 \\
33 \\
31 \\
30 \\
29\end{array}$ & $\begin{array}{c}11 \\
10 \\
9.9 \\
9.4 \\
8.6\end{array}$ & $\begin{array}{l}18 \\
16 \\
14 \\
12 \\
11\end{array}$ & $\begin{array}{r}0.68 \\
.62 \\
4.3 \\
38\end{array}$ & $\begin{array}{r}0.87 \\
.86 \\
.85 \\
1.6 \\
2.4\end{array}$ & $\begin{array}{l}5.3 \\
4.5 \\
3.8 \\
3.3 \\
2.7\end{array}$ & $\begin{array}{l}12 \\
20 \\
30 \\
31 \\
19\end{array}$ & $\begin{array}{r}2.4 \\
2.7 \\
2.3 \\
1.5 \\
.99\end{array}$ & $\begin{array}{l}-- \\
--- \\
-- \\
-- \\
--\end{array}$ \\
\hline $\begin{array}{l}.77 \\
.70 \\
.65 \\
.91 \\
.82\end{array}$ & $\begin{array}{l}3.3 \\
3.2 \\
2.5 \\
2.1 \\
2.3\end{array}$ & $\begin{array}{l}.80 \\
.77 \\
.75 \\
.69 \\
.62\end{array}$ & $\begin{array}{r}27 \\
26 \\
25 \\
23 \\
---\end{array}$ & $\begin{array}{l}7.8 \\
6.8 \\
6.1 \\
5.7 \\
5.2\end{array}$ & $\begin{array}{r}11 \\
9.7 \\
8.9 \\
8.2 \\
7.6\end{array}$ & $\begin{array}{l}28 \\
22 \\
17 \\
14 \\
13\end{array}$ & $\begin{array}{l}.86 \\
.77 \\
.65 \\
.60 \\
.58\end{array}$ & $\begin{array}{l}2.2 \\
1.6 \\
1.3 \\
1.2 \\
1.1\end{array}$ & $\begin{array}{r}11 \\
7.7 \\
5.4 \\
3.4 \\
2.7\end{array}$ & $\begin{array}{l}.88 \\
.80 \\
.78 \\
.70 \\
.58\end{array}$ & $\begin{array}{l}--- \\
--- \\
-- \\
--\end{array}$ \\
\hline $\begin{array}{l}4.3 \\
8.5 \\
7.7 \\
5.6 \\
5.9\end{array}$ & $\begin{array}{l}2.1 \\
1.6 \\
1.3 \\
1.0 \\
1.0\end{array}$ & $\begin{array}{l}3.2 \\
80 \\
49 \\
40 \\
43\end{array}$ & $\begin{array}{r}--- \\
32 \\
28 \\
18 \\
15\end{array}$ & $\begin{array}{r}4.9 \\
4.9 \\
14 \\
23 \\
15\end{array}$ & $\begin{array}{l}6.9 \\
6.0 \\
5.5 \\
4.8 \\
4.0\end{array}$ & $\begin{array}{l}12 \\
11 \\
10 \\
10 \\
17\end{array}$ & $\begin{array}{l}.50 \\
.40 \\
.25 \\
.06 \\
.04\end{array}$ & $\begin{array}{r}1.1 \\
1.1 \\
1.0 \\
.85 \\
.70\end{array}$ & $\begin{array}{l}3.4 \\
3.8 \\
4.1 \\
4.8 \\
5.0\end{array}$ & $\begin{array}{r}.57 \\
1.1 \\
1.8 \\
.84 \\
.79\end{array}$ & $\begin{array}{l}-- \\
-- \\
-- \\
-- \\
--\end{array}$ \\
\hline $\begin{array}{l}49 \\
65 \\
49 \\
35 \\
26\end{array}$ & $\begin{array}{l}1.8 \\
1.8 \\
1.6 \\
1.3 \\
1.9\end{array}$ & $\begin{array}{l}42 \\
42 \\
49 \\
43 \\
59\end{array}$ & $\begin{array}{l}12 \\
11 \\
11 \\
13 \\
13\end{array}$ & $\begin{array}{c}11 \\
10 \\
9.6 \\
9.3 \\
9.0\end{array}$ & $\begin{array}{l}3.2 \\
2.5 \\
2.0 \\
1.5 \\
1.5\end{array}$ & $\begin{array}{r}13 \\
11 \\
9.3 \\
8.2 \\
7.4\end{array}$ & $\begin{array}{l}.02 \\
.01 \\
.00 \\
.00 \\
.00\end{array}$ & $\begin{array}{r}.63 \\
.57 \\
.53 \\
.50 \\
.47\end{array}$ & $\begin{array}{l}4.7 \\
3.9 \\
2.9 \\
2.9 \\
5.3\end{array}$ & $\begin{array}{l}.74 \\
.64 \\
.59 \\
-.-2 \\
-.-\end{array}$ & $\begin{array}{l}--- \\
--- \\
--- \\
---\end{array}$ \\
\hline $\begin{array}{l}17 \\
32 \\
40 \\
34 \\
25\end{array}$ & $\begin{array}{r}2.8 \\
2.3 \\
1.9 \\
3.5 \\
11\end{array}$ & $\begin{array}{l}94 \\
67 \\
45 \\
37 \\
34\end{array}$ & $\begin{array}{l}25 \\
25 \\
21 \\
17 \\
18\end{array}$ & $\begin{array}{l}9.1 \\
30 \\
41 \\
37 \\
34\end{array}$ & $\begin{array}{l}2.9 \\
1.5 \\
1.0 \\
.89 \\
.86\end{array}$ & $\begin{array}{l}7.0 \\
6.4 \\
6.0 \\
5.8 \\
4.7\end{array}$ & $\begin{array}{l}.00 \\
26 \\
18 \\
11\end{array}$ & $\begin{array}{r}.43 \\
.37 \\
2.35 \\
12.6\end{array}$ & $\begin{array}{r}12 \\
13 \\
12 \\
9.5 \\
7.4\end{array}$ & $\begin{array}{l}--- \\
--- \\
--- \\
--\end{array}$ & $\begin{array}{l}-- \\
-- \\
-- \\
-- \\
--\end{array}$ \\
\hline $\begin{array}{l}26 \\
11 \\
8.5 \\
7.1 \\
6.1 \\
5.4\end{array}$ & $\begin{array}{l}3.9 \\
1.9 \\
1.2 \\
2.1 \\
1.9 \\
---\end{array}$ & $\begin{array}{l}31 \\
30 \\
28 \\
43 \\
50 \\
40\end{array}$ & $\begin{array}{l}17 \\
16 \\
15 \\
13 \\
12 \\
11\end{array}$ & $\begin{array}{l}28 \\
22 \\
25 \\
21 \\
-- \\
--\end{array}$ & $\begin{array}{r}.85 \\
1.0 \\
.96 \\
.85 \\
.79 \\
.75\end{array}$ & $\begin{array}{l}3.7 \\
2.7 \\
1.9 \\
1.4 \\
.99 \\
-.--\end{array}$ & $\begin{array}{l}12 \\
13 \\
10 \\
7.9 \\
6.5 \\
6.0\end{array}$ & $\begin{array}{r}14 \\
11 \\
8.8 \\
4.4 \\
4.9 \\
-\end{array}$ & $\begin{array}{l}5.6 \\
4.2 \\
2.8 \\
1.8 \\
1.3 \\
1.9\end{array}$ & $\begin{array}{l}--- \\
-\cdots \\
-\cdots \\
---\end{array}$ & $\begin{array}{r}--- \\
--- \\
--- \\
56 \\
43 \\
---\end{array}$ \\
\hline
\end{tabular}


APPENDIX III--Daily discharge--Continued

[Mean values in cubic feet per second]

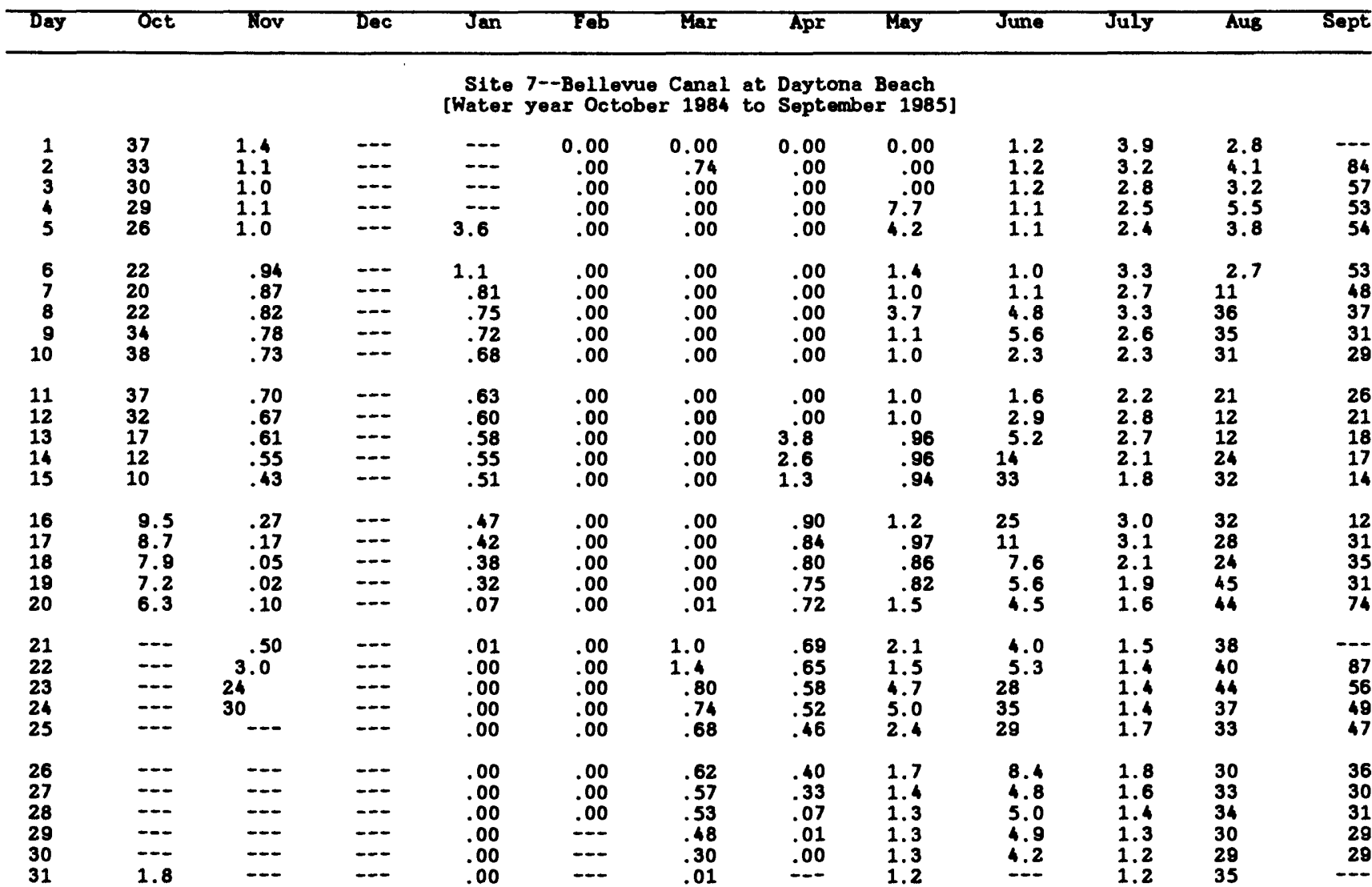

Site 11--B-19 Canal at Willow Run Boulevard at Port Orange [Water year October 1982 to September 1983]

\begin{tabular}{|c|c|c|c|c|c|c|c|c|c|c|c|c|}
\hline $\begin{array}{l}1 \\
2 \\
3 \\
4 \\
5\end{array}$ & $\begin{array}{l}\ldots- \\
-\ldots \\
-\cdots \\
-\cdots\end{array}$ & $\begin{array}{l}-- \\
-- \\
-- \\
--\end{array}$ & $\begin{array}{l}15 \\
14 \\
14 \\
14 \\
14\end{array}$ & $\begin{array}{l}15 \\
15 \\
15 \\
15 \\
14\end{array}$ & $\begin{array}{l}18 \\
32 \\
41 \\
34 \\
29\end{array}$ & $\begin{array}{l}19 \\
21 \\
19 \\
18 \\
18\end{array}$ & $\begin{array}{l}-- \\
-- \\
-- \\
-- \\
--\end{array}$ & $\begin{array}{l}25 \\
24 \\
23 \\
24 \\
23\end{array}$ & $\begin{array}{l}31 \\
30 \\
28 \\
28 \\
31\end{array}$ & $\begin{array}{l}31 \\
32 \\
31 \\
30 \\
29\end{array}$ & $\begin{array}{l}33 \\
32 \\
23 \\
21 \\
20\end{array}$ & $\begin{array}{l}18 \\
31 \\
30 \\
26 \\
21\end{array}$ \\
\hline $\begin{array}{r}6 \\
7 \\
8 \\
9 \\
10\end{array}$ & 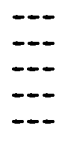 & $\begin{array}{l}--- \\
-\cdots \\
-\cdots \\
---\end{array}$ & $\begin{array}{l}13 \\
20 \\
21 \\
20 \\
22\end{array}$ & $\begin{array}{l}14 \\
14 \\
14 \\
14 \\
14\end{array}$ & $\begin{array}{l}30 \\
34 \\
30 \\
27 \\
26\end{array}$ & $\begin{array}{l}18 \\
24 \\
31 \\
30 \\
26\end{array}$ & $\begin{array}{l}-- \\
--- \\
--- \\
-- \\
--\end{array}$ & $\begin{array}{l}23 \\
22 \\
21 \\
21 \\
20\end{array}$ & $\begin{array}{l}34 \\
37 \\
54 \\
54 \\
49\end{array}$ & $\begin{array}{l}29 \\
28 \\
28 \\
27 \\
26\end{array}$ & $\begin{array}{l}18 \\
20 \\
19 \\
22 \\
34\end{array}$ & $\begin{array}{l}19 \\
17 \\
16 \\
15 \\
14\end{array}$ \\
\hline $\begin{array}{l}11 \\
12 \\
13 \\
14 \\
15\end{array}$ & 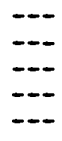 & $\begin{array}{l}18 \\
17 \\
16 \\
16 \\
16\end{array}$ & $\begin{array}{l}24 \\
22 \\
21 \\
20 \\
19\end{array}$ & $\begin{array}{l}14 \\
14 \\
14 \\
14 \\
15\end{array}$ & $\begin{array}{l}28 \\
28 \\
52 \\
56 \\
44\end{array}$ & $\begin{array}{l}23 \\
21 \\
20 \\
20 \\
26\end{array}$ & $\begin{array}{l}-- \\
-- \\
-- \\
-- \\
--\end{array}$ & $\begin{array}{l}20 \\
20 \\
20 \\
20 \\
20\end{array}$ & $\begin{array}{l}44 \\
40 \\
37 \\
37 \\
35\end{array}$ & $\begin{array}{l}26 \\
25 \\
25 \\
24 \\
23\end{array}$ & $\begin{array}{l}28 \\
25 \\
29 \\
71 \\
55\end{array}$ & $\begin{array}{l}13 \\
13 \\
12 \\
11 \\
11\end{array}$ \\
\hline $\begin{array}{l}16 \\
17 \\
18 \\
19 \\
20\end{array}$ & $\overline{-}$ & $\begin{array}{l}16 \\
16 \\
15 \\
15 \\
15\end{array}$ & $\begin{array}{l}19 \\
19 \\
18 \\
18 \\
17\end{array}$ & $\begin{array}{l}16 \\
14 \\
15 \\
14 \\
17\end{array}$ & $\begin{array}{l}38 \\
37 \\
33 \\
30 \\
28\end{array}$ & $\begin{array}{l}61 \\
66 \\
61 \\
49 \\
41\end{array}$ & $\begin{array}{l}-- \\
--- \\
--- \\
--- \\
38\end{array}$ & $\begin{array}{l}20 \\
21 \\
21 \\
21 \\
20\end{array}$ & $\begin{array}{l}33 \\
31 \\
30 \\
29 \\
28\end{array}$ & $\begin{array}{l}23 \\
23 \\
23 \\
23 \\
23\end{array}$ & $\begin{array}{l}32 \\
25 \\
21 \\
19 \\
17\end{array}$ & $\begin{array}{l}11 \\
15 \\
17 \\
16 \\
17\end{array}$ \\
\hline $\begin{array}{l}21 \\
22 \\
23 \\
24 \\
25\end{array}$ & $\begin{array}{l}\ldots- \\
\ldots- \\
\ldots- \\
\ldots-\end{array}$ & $\begin{array}{l}15 \\
15 \\
15 \\
16 \\
15\end{array}$ & $\begin{array}{l}16 \\
16 \\
16 \\
16 \\
15\end{array}$ & $\begin{array}{l}20 \\
19 \\
18 \\
19 \\
18\end{array}$ & $\begin{array}{l}25 \\
24 \\
23 \\
22 \\
22\end{array}$ & $\begin{array}{l}43 \\
39 \\
34 \\
35 \\
33\end{array}$ & $\begin{array}{l}35 \\
31 \\
37 \\
54 \\
47\end{array}$ & $\begin{array}{l}20 \\
19 \\
19 \\
19 \\
19\end{array}$ & $\begin{array}{l}33 \\
42 \\
43 \\
44 \\
43\end{array}$ & $\begin{array}{l}24 \\
23 \\
23 \\
22 \\
22\end{array}$ & $\begin{array}{l}16 \\
15 \\
14 \\
13 \\
13\end{array}$ & $\begin{array}{l}17 \\
16 \\
14 \\
13 \\
23\end{array}$ \\
\hline $\begin{array}{l}26 \\
27 \\
28 \\
29 \\
30 \\
31\end{array}$ & $\begin{array}{l}-- \\
-\cdots \\
\cdots- \\
-\cdots \\
-\cdots\end{array}$ & $\begin{array}{r}15 \\
15 \\
15 \\
16 \\
15 \\
--\end{array}$ & $\begin{array}{l}15 \\
15 \\
15 \\
15 \\
15 \\
15\end{array}$ & $\begin{array}{l}18 \\
20 \\
21 \\
20 \\
19 \\
19\end{array}$ & \begin{tabular}{r}
21 \\
20 \\
19 \\
- \\
\hdashline- \\
$-\ldots$
\end{tabular} & $\begin{array}{r}28 \\
30 \\
31 \\
31 \\
- \\
-\end{array}$ & $\begin{array}{r}40 \\
34 \\
30 \\
28 \\
26 \\
-\end{array}$ & $\begin{array}{l}21 \\
20 \\
20 \\
24 \\
32 \\
33\end{array}$ & $\begin{array}{r}40 \\
38 \\
35 \\
34 \\
32 \\
-\end{array}$ & $\begin{array}{l}26 \\
25 \\
24 \\
27 \\
28 \\
28\end{array}$ & $\begin{array}{l}13 \\
12 \\
12 \\
11 \\
11 \\
11\end{array}$ & $\begin{array}{l}18 \\
17 \\
15 \\
14 \\
13\end{array}$ \\
\hline
\end{tabular}




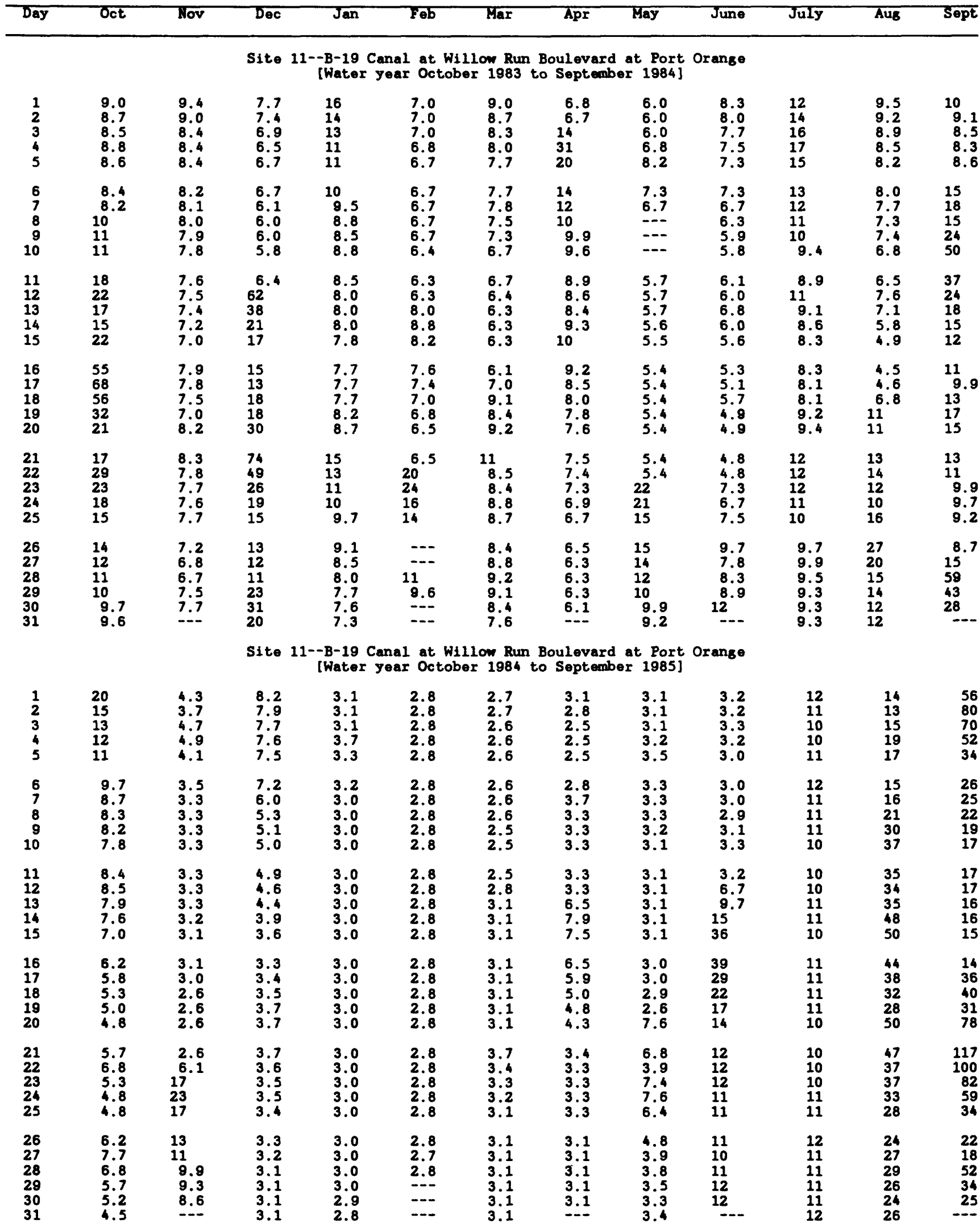


APPENDIX III--Daily discharge--Continued

[Mean values in cubic feet per second]

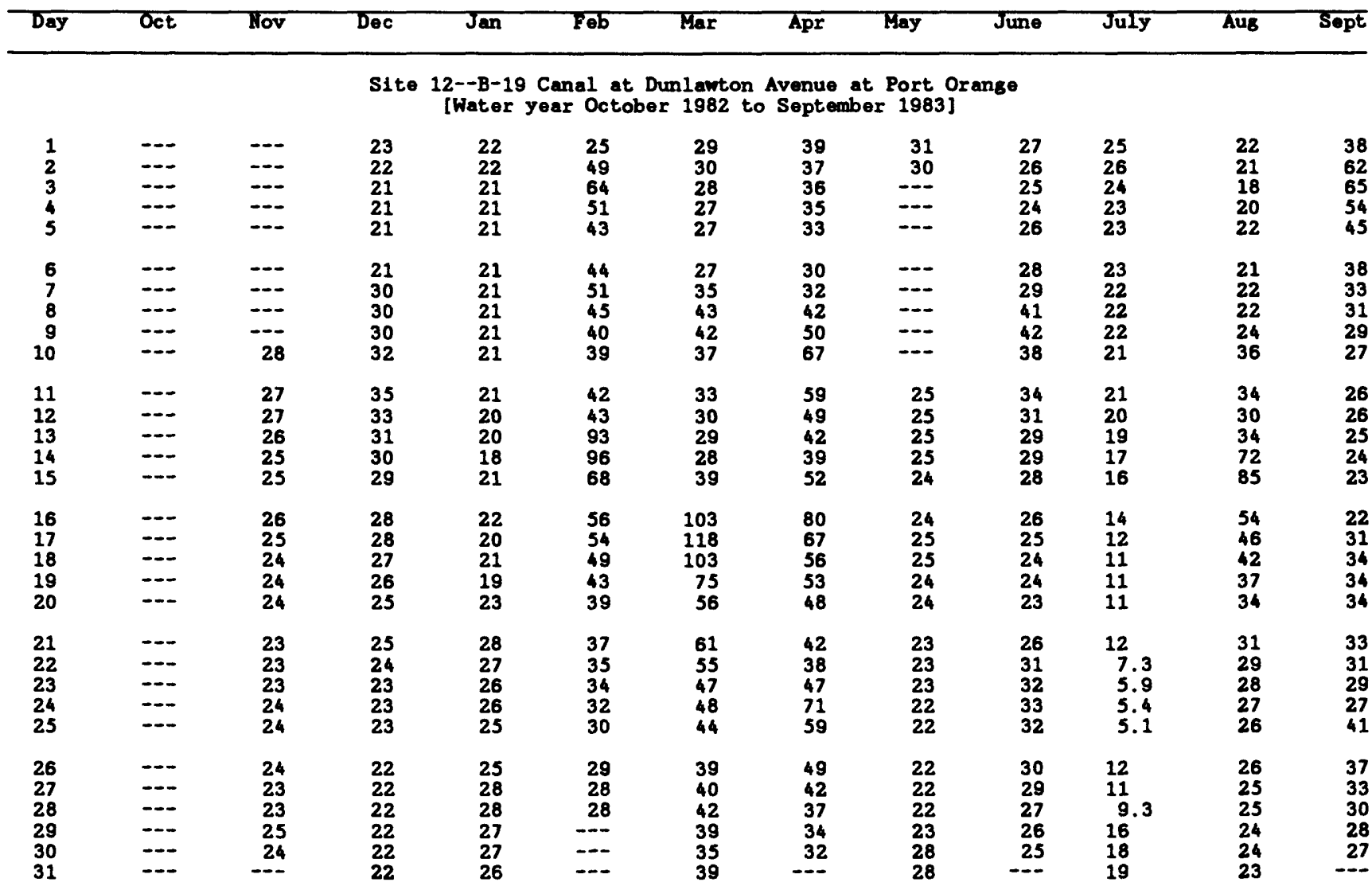

Site 12--B-19 Canal at Dunlawton Avenue at Port Orange [Water year October 1983 to September 1984]

\begin{tabular}{|c|c|c|c|c|c|c|c|c|c|c|c|}
\hline $\begin{array}{l}1 \\
2 \\
3 \\
4 \\
5\end{array}$ & $\begin{array}{l}25 \\
24 \\
24 \\
24 \\
24\end{array}$ & $\begin{array}{l}32 \\
31 \\
30 \\
30 \\
29\end{array}$ & $\begin{array}{l}27 \\
26 \\
26 \\
25 \\
26\end{array}$ & $\begin{array}{l}56 \\
50 \\
46 \\
43 \\
41\end{array}$ & $\begin{array}{l}30 \\
30 \\
30 \\
29 \\
29\end{array}$ & $\begin{array}{l}36 \\
35 \\
34 \\
33 \\
32\end{array}$ & $\begin{array}{l}25 \\
24 \\
41 \\
96 \\
68\end{array}$ & $\begin{array}{l}25 \\
24 \\
23 \\
25 \\
28\end{array}$ & $\begin{array}{l}29 \\
27 \\
26 \\
26 \\
25\end{array}$ & $\begin{array}{l}42 \\
47 \\
47 \\
49 \\
45\end{array}$ & $\begin{array}{l}30 \\
30 \\
27 \\
26 \\
25\end{array}$ \\
\hline $\begin{array}{r}6 \\
7 \\
8 \\
9 \\
10\end{array}$ & $\begin{array}{l}23 \\
22 \\
29 \\
32 \\
32\end{array}$ & $\begin{array}{l}29 \\
29 \\
28 \\
28 \\
28\end{array}$ & $\begin{array}{l}26 \\
25 \\
24 \\
25 \\
24\end{array}$ & $\begin{array}{l}40 \\
38 \\
36 \\
35 \\
36\end{array}$ & $\begin{array}{l}28 \\
28 \\
27 \\
27 \\
27\end{array}$ & $\begin{array}{l}32 \\
32 \\
30 \\
30 \\
29\end{array}$ & $\begin{array}{l}51 \\
43 \\
38 \\
37 \\
36\end{array}$ & $\begin{array}{l}26 \\
26 \\
24 \\
24 \\
23\end{array}$ & $\begin{array}{l}24 \\
24 \\
23 \\
22 \\
22\end{array}$ & $\begin{array}{l}39 \\
36 \\
36 \\
31 \\
30\end{array}$ & $\begin{array}{l}25 \\
24 \\
23 \\
24 \\
23\end{array}$ \\
\hline $\begin{array}{l}11 \\
12 \\
13 \\
14 \\
15\end{array}$ & $\begin{array}{l}50 \\
63 \\
51 \\
43 \\
63\end{array}$ & $\begin{array}{l}27 \\
26 \\
26 \\
25 \\
25\end{array}$ & $\begin{array}{r}25 \\
154 \\
102 \\
66 \\
55\end{array}$ & $\begin{array}{l}-- \\
-- \\
-- \\
-- \\
--\end{array}$ & $\begin{array}{l}27 \\
27 \\
34 \\
37 \\
35\end{array}$ & $\begin{array}{l}29 \\
28 \\
29 \\
28 \\
27\end{array}$ & $\begin{array}{l}34 \\
33 \\
32 \\
34 \\
38\end{array}$ & $\begin{array}{l}22 \\
22 \\
22 \\
23 \\
22\end{array}$ & $\begin{array}{l}22 \\
24 \\
24 \\
22 \\
22\end{array}$ & $\begin{array}{l}28 \\
31 \\
29 \\
27 \\
26\end{array}$ & $\begin{array}{l}24 \\
24 \\
25 \\
22 \\
21\end{array}$ \\
\hline $\begin{array}{l}16 \\
17 \\
18 \\
19 \\
20\end{array}$ & $\begin{array}{r}144 \\
183 \\
139 \\
86 \\
62\end{array}$ & $\begin{array}{l}28 \\
28 \\
27 \\
25 \\
29\end{array}$ & $\begin{array}{l}48 \\
45 \\
56 \\
55 \\
81\end{array}$ & $\begin{array}{l}--- \\
-- \\
--- \\
-- \\
--\end{array}$ & $\begin{array}{l}33 \\
32 \\
31 \\
30 \\
29\end{array}$ & $\begin{array}{l}27 \\
26 \\
26 \\
26 \\
27\end{array}$ & $\begin{array}{l}34 \\
32 \\
31 \\
30 \\
30\end{array}$ & $\begin{array}{l}21 \\
22 \\
21 \\
20 \\
20\end{array}$ & $\begin{array}{l}21 \\
21 \\
23 \\
21 \\
21\end{array}$ & $\begin{array}{l}26 \\
25 \\
25 \\
28 \\
28\end{array}$ & $\begin{array}{l}21 \\
21 \\
24 \\
30 \\
33\end{array}$ \\
\hline $\begin{array}{l}21 \\
22 \\
23 \\
24 \\
25\end{array}$ & $\begin{array}{l}52 \\
78 \\
68 \\
55 \\
48\end{array}$ & $\begin{array}{l}29 \\
28 \\
27 \\
27 \\
27\end{array}$ & $\begin{array}{r}187 \\
129 \\
80 \\
61 \\
51\end{array}$ & $\begin{array}{l}-- \\
-- \\
--\infty \\
--\infty \\
--\infty\end{array}$ & $\begin{array}{l}29 \\
66 \\
81 \\
56 \\
48\end{array}$ & $\begin{array}{l}29 \\
27 \\
26 \\
26 \\
26\end{array}$ & $\begin{array}{l}28 \\
28 \\
29 \\
27 \\
27\end{array}$ & $\begin{array}{l}22 \\
21 \\
51 \\
54 \\
42\end{array}$ & $\begin{array}{l}21 \\
22 \\
26 \\
26 \\
29\end{array}$ & $\begin{array}{l}33 \\
34 \\
35 \\
33 \\
32\end{array}$ & $\begin{array}{l}34 \\
40 \\
34 \\
31 \\
40\end{array}$ \\
\hline $\begin{array}{l}26 \\
27 \\
28 \\
29 \\
30 \\
31\end{array}$ & $\begin{array}{l}43 \\
41 \\
37 \\
35 \\
34 \\
33\end{array}$ & $\begin{array}{r}26 \\
26 \\
25 \\
27 \\
27 \\
---\end{array}$ & $\begin{array}{l}47 \\
44 \\
42 \\
73 \\
96 \\
68\end{array}$ & $\begin{array}{l}37 \\
36 \\
35 \\
33 \\
32 \\
31\end{array}$ & \begin{tabular}{r}
42 \\
41 \\
43 \\
38 \\
\hdashline- \\
-
\end{tabular} & $\begin{array}{l}26 \\
27 \\
27 \\
27 \\
25 \\
25\end{array}$ & $\begin{array}{r}26 \\
25 \\
26 \\
24 \\
24 \\
-\end{array}$ & $\begin{array}{l}41 \\
40 \\
37 \\
33 \\
32 \\
30\end{array}$ & $\begin{array}{r}31 \\
27 \\
28 \\
30 \\
38 \\
-\end{array}$ & $\begin{array}{l}30 \\
29 \\
29 \\
28 \\
28 \\
28\end{array}$ & $\begin{array}{l}58 \\
51 \\
41 \\
37 \\
34 \\
32\end{array}$ \\
\hline
\end{tabular}


APPENDIX III--Daily discharge--Continued [Mean values in cubic feet per second]

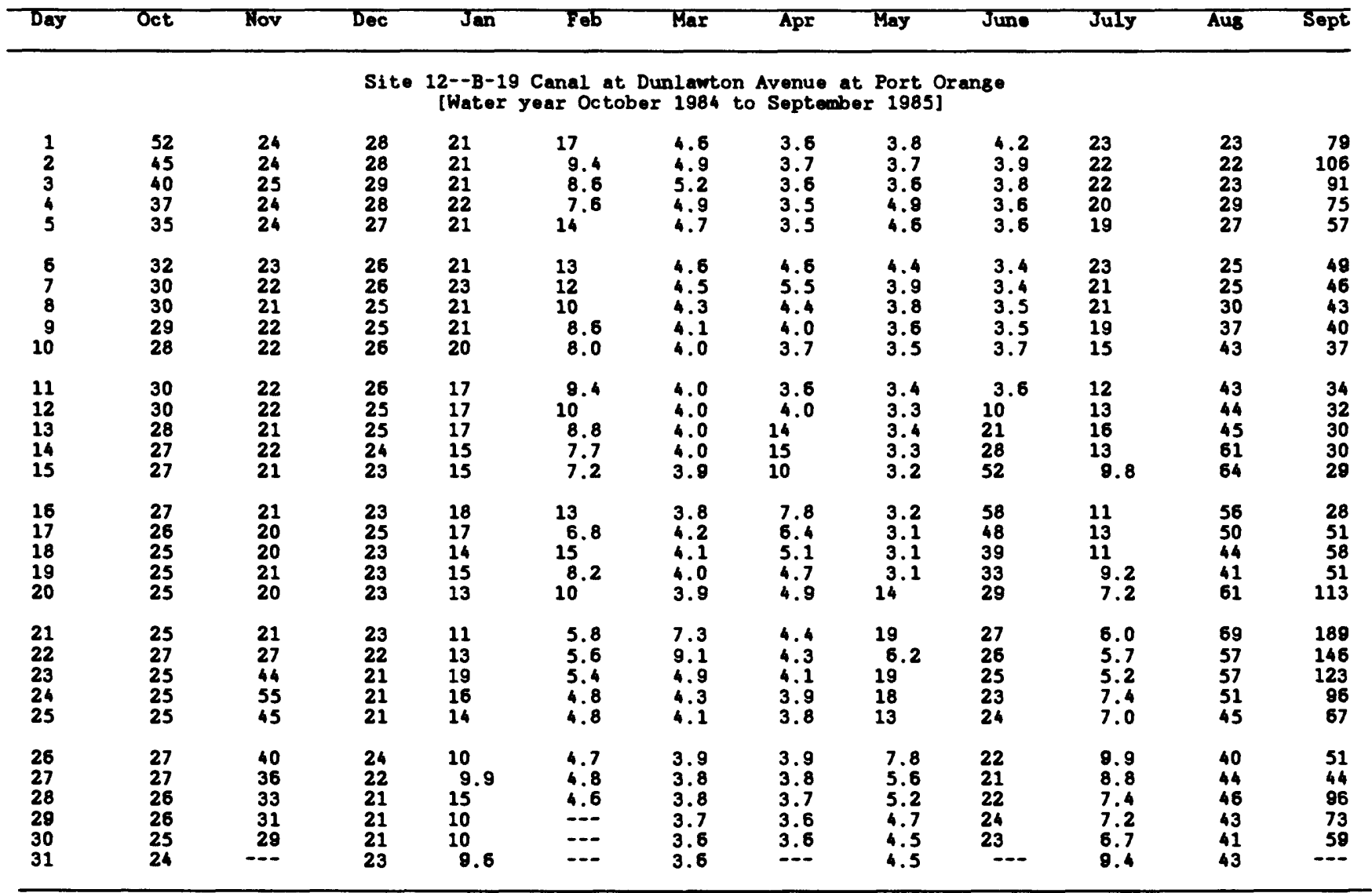




\section{Appendix IV}


APPENDIX IV--Quality of water analyses

[L denotes low flow sample; $C$ denotes a composite sample; $S$ denotes calculated by summing components]

\begin{tabular}{|c|c|c|c|c|c|c|c|c|c|c|c|}
\hline $\begin{array}{l}\text { Site } \\
\text { No. }\end{array}$ & $\begin{array}{c}\text { Date } \\
\text { of } \\
\text { sample }\end{array}$ & $\begin{array}{l}\text { Spe- } \\
\text { cific } \\
\text { con- } \\
\text { duc- } \\
\text { tance } \\
\text { Lab } \\
(\mu \mathrm{S} / \mathrm{cm})\end{array}$ & $\begin{array}{l}\text { Tem- } \\
\text { per- } \\
\text { ature } \\
\left({ }^{\circ} \mathrm{C}\right)\end{array}$ & $\begin{array}{c}\text { pH } \\
\text { Lab } \\
\text { (stand- } \\
\text { ard } \\
\text { units) }\end{array}$ & $\begin{array}{l}\text { Color } \\
\text { (plat- } \\
\text { inum- } \\
\text { cobalt } \\
\text { units) }\end{array}$ & $\begin{array}{l}\text { Hard- } \\
\text { ness } \\
\text { (mg/L } \\
\text { as } \\
\mathrm{CaCO}_{3} \text { ) }\end{array}$ & $\begin{array}{l}\text { Hard- } \\
\text { ness } \\
\text { noncar- } \\
\text { bonate } \\
\text { (mg/L } \\
\text { as } \\
\mathrm{CaCO}_{3} \text { ) }\end{array}$ & $\begin{array}{l}\text { Cal- } \\
\text { cium } \\
\text { dis- } \\
\text { solved } \\
\text { (mg/L } \\
\text { as Ca) }\end{array}$ & $\begin{array}{l}\text { Magne- } \\
\text { sium, } \\
\text { dis- } \\
\text { solved } \\
\text { (mg/L } \\
\text { as mg) }\end{array}$ & $\begin{array}{l}\text { So- } \\
\text { dium } \\
\text { dis- } \\
\text { solved } \\
\text { (mg/L } \\
\text { as } \mathrm{Na} \text { ) }\end{array}$ & $\begin{array}{l}\text { Potas- } \\
\text { sium, } \\
\text { dis-- } \\
\text { solved } \\
\text { (mg/L } \\
\text { as } K \text { ) }\end{array}$ \\
\hline 1 & $\begin{array}{l}03-09-84 L \\
09-28-84\end{array}$ & $\begin{array}{l}77 \\
68\end{array}$ & 14.5 & $\begin{array}{l}6.50 \\
6.50\end{array}$ & $\begin{array}{l}280 \\
160\end{array}$ & $\begin{array}{l}27 \\
22\end{array}$ & $\begin{array}{r}11 \\
8\end{array}$ & $\begin{array}{l}8.7 \\
7.3\end{array}$ & $\begin{array}{l}1.3 \\
1.0\end{array}$ & $\begin{array}{l}7.1 \\
5.2\end{array}$ & $\begin{array}{l}.40 \\
.90\end{array}$ \\
\hline 2 & $\begin{array}{l}08-18-83 L \\
12-12-83 C \\
12-21-83 \\
03-15-84 L \\
09-28-84\end{array}$ & $\begin{array}{l}397 \\
223 \\
121 \\
431 \\
116\end{array}$ & $\begin{array}{r}-- \\
-- \\
19.0 \\
--\end{array}$ & $\begin{array}{l}7.40 \\
7.00 \\
6.10 \\
7.20 \\
6.80\end{array}$ & $\begin{array}{r}70 \\
65 \\
230 \\
95 \\
140\end{array}$ & $\begin{array}{r}180 \\
93 \\
48 \\
190 \\
43\end{array}$ & $\begin{array}{l}32 \\
18 \\
22 \\
32 \\
14\end{array}$ & $\begin{array}{l}65 \\
34 \\
16 \\
70 \\
15\end{array}$ & $\begin{array}{l}3.4 \\
2.0 \\
1.9 \\
3.3 \\
1.4\end{array}$ & $\begin{array}{c}17 \\
9.0 \\
8.4 \\
18 \\
6.7\end{array}$ & $\begin{array}{l}.30 \\
-- \\
.70 \\
.60 \\
.90\end{array}$ \\
\hline 3 & $\begin{array}{l}08-18-83 L \\
12-21-83 \\
02-22-84 \\
03-06-84 L \\
04-04-84 \\
05-23-84 C\end{array}$ & $\begin{array}{r}305 \\
73 \\
213 \\
246 \\
54 \\
67\end{array}$ & $\begin{array}{r}32.5 \\
-. \\
-- \\
26.0 \\
-.-\end{array}$ & $\begin{array}{l}7.40 \\
5.90 \\
6.80 \\
6.60 \\
6.60 \\
7.00\end{array}$ & $\begin{array}{r}50 \\
190 \\
50 \\
60 \\
40 \\
30\end{array}$ & $\begin{array}{r}120 \\
26 \\
83 \\
84 \\
25 \\
27\end{array}$ & $\begin{array}{r}35 \\
10 \\
23 \\
40 \\
6 \\
8\end{array}$ & $\begin{array}{l}38 \\
8.1 \\
26 \\
22 \\
8.7 \\
8.8\end{array}$ & $\begin{array}{l}6.2 \\
1.3 \\
4.3 \\
7.1 \\
.76 \\
1.1\end{array}$ & $\begin{array}{l}16 \\
6.4 \\
12 \\
17 \\
2.2 \\
2.6\end{array}$ & $\begin{array}{l}.10 \\
.50 \\
.30 \\
.30 \\
.50 \\
.40\end{array}$ \\
\hline 4 & $\begin{array}{l}08-10-83 L \\
12-21-83 \\
02-22-84 C \\
03-06-84 L \\
09-28-84\end{array}$ & $\begin{array}{l}175 \\
114 \\
290 \\
395 \\
119\end{array}$ & $\begin{array}{r}26.5 \\
-.- \\
22.0 \\
--\end{array}$ & $\begin{array}{r}7.00 \\
6.40 \\
7.40 \\
7.00 \\
6.90\end{array}$ & $\begin{array}{r}30 \\
170 \\
75 \\
110 \\
120\end{array}$ & $\begin{array}{r}75 \\
49 \\
120 \\
150 \\
46\end{array}$ & $\begin{array}{r}9 \\
12 \\
13 \\
19 \\
10\end{array}$ & $\begin{array}{l}27 \\
17 \\
42 \\
50 \\
16\end{array}$ & $\begin{array}{l}1.7 \\
1.5 \\
2.8 \\
6.4 \\
1.4\end{array}$ & $\begin{array}{l}6.4 \\
7.0 \\
11 \\
22 \\
6.5\end{array}$ & $\begin{array}{l}.40 \\
.50 \\
.90 \\
.70 \\
.70\end{array}$ \\
\hline 5 & $\begin{array}{l}08-18-83 L \\
12-21-83 \\
03-19-84 L \\
09-28-84\end{array}$ & $\begin{array}{l}64 \\
52 \\
49 \\
59\end{array}$ & $\begin{array}{r}24.5 \\
20.0 \\
--\end{array}$ & $\begin{array}{l}4.30 \\
4.00 \\
4.00 \\
4.40\end{array}$ & $\begin{array}{l}360 \\
300 \\
300 \\
250\end{array}$ & $\begin{array}{r}11 \\
8 \\
8 \\
7\end{array}$ & $\begin{array}{l}10 \\
-- \\
--\end{array}$ & $\begin{array}{l}2.9 \\
1.8 \\
2.1 \\
1.7\end{array}$ & $\begin{array}{l}.90 \\
.84 \\
.78 \\
.65\end{array}$ & $\begin{array}{l}6.0 \\
5.7 \\
6.2 \\
4.1\end{array}$ & $\begin{array}{r}.10 \\
.20 \\
<.10 \\
.20\end{array}$ \\
\hline 6 & $\begin{array}{l}08-10-83 L \\
12-20-83 C \\
12-21-83 \\
12-22-83 \\
02-22-84 \\
03-09-84 L\end{array}$ & $\begin{array}{l}191 \\
171 \\
140 \\
218 \\
385 \\
492\end{array}$ & $\begin{array}{r}28.0 \\
-- \\
-- \\
-- \\
-- \\
13.0\end{array}$ & $\begin{array}{l}6.90 \\
6.80 \\
6.60 \\
6.70 \\
6.90 \\
7.50\end{array}$ & $\begin{array}{r}40 \\
100 \\
150 \\
140 \\
70 \\
85\end{array}$ & $\begin{array}{r}69 \\
66 \\
57 \\
87 \\
150 \\
190\end{array}$ & $\begin{array}{r}11 \\
8 \\
12 \\
14 \\
6 \\
17\end{array}$ & $\begin{array}{l}24 \\
23 \\
20 \\
30 \\
53 \\
68\end{array}$ & $\begin{array}{l}2.2 \\
2.1 \\
1.8 \\
2.9 \\
3.7 \\
6.0\end{array}$ & $\begin{array}{l}11 \\
8.5 \\
8.5 \\
11 \\
19 \\
27\end{array}$ & $\begin{array}{r}1.2 \\
1.8 \\
1.4 \\
1.9 \\
1.4 \\
.90\end{array}$ \\
\hline 7 & $\begin{array}{l}08-10-83 L \\
12-21-83 \\
02-22-84 \\
03-19-84 L \\
04-04-84 \\
05-23-84 C\end{array}$ & $\begin{array}{r}176 \\
92 \\
196 \\
430 \\
139 \\
114\end{array}$ & $\begin{array}{r}27.0 \\
-- \\
-- \\
-- \\
-- \\
--\end{array}$ & $\begin{array}{l}7.10 \\
6.40 \\
6.80 \\
7.10 \\
6.90 \\
7.20\end{array}$ & $\begin{array}{r}35 \\
150 \\
70 \\
60 \\
35 \\
40\end{array}$ & $\begin{array}{r}-- \\
40 \\
82 \\
180 \\
60 \\
40\end{array}$ & $\begin{array}{r}-- \\
5 \\
0 \\
8 \\
4 \\
0\end{array}$ & $\begin{array}{l}-- \\
14 \\
30 \\
62 \\
22 \\
14\end{array}$ & $\begin{array}{l}1.6 \\
1.3 \\
1.6 \\
4.9 \\
1.3 \\
1.1\end{array}$ & $\begin{array}{c}5.7 \\
5.6 \\
6.8 \\
22 \\
5.7 \\
5.5\end{array}$ & $\begin{array}{r}.30 \\
.70 \\
1.2 \\
.30 \\
.60 \\
.80\end{array}$ \\
\hline 8 & $\begin{array}{l}12-21-83 \\
03-19-84 \mathrm{~L} \\
09-28-84\end{array}$ & $\begin{array}{l}52 \\
50 \\
53\end{array}$ & 20.5 & $\begin{array}{l}4.00 \\
7.20 \\
4.60\end{array}$ & $\begin{array}{l}300 \\
300 \\
300\end{array}$ & $\begin{array}{r}9 \\
12 \\
9\end{array}$ & 10 & $\begin{array}{l}2.2 \\
3.5 \\
2.4\end{array}$ & $\begin{array}{l}.82 \\
.86 \\
.71\end{array}$ & $\begin{array}{l}5.6 \\
6.9 \\
4.2\end{array}$ & $\begin{array}{r}.20 \\
<.10 \\
.30\end{array}$ \\
\hline 9 & $\begin{array}{l}08-18-83 L \\
12-21-83 \\
03-09-84 L \\
09-28-84\end{array}$ & $\begin{array}{r}145 \\
72 \\
93 \\
59\end{array}$ & 13.5 & $\begin{array}{l}6.40 \\
5.80 \\
6.30 \\
6.10\end{array}$ & $\begin{array}{l}280 \\
300 \\
320 \\
280\end{array}$ & $\begin{array}{l}50 \\
23 \\
33 \\
18\end{array}$ & $\begin{array}{r}-- \\
7 \\
10 \\
9\end{array}$ & $\begin{array}{c}15 \\
6.6 \\
10 \\
5.5\end{array}$ & $\begin{array}{l}2.9 \\
1.5 \\
2.0 \\
1.0\end{array}$ & $\begin{array}{l}8.8 \\
6.5 \\
7.8 \\
4.7\end{array}$ & $\begin{array}{l}5.2 \\
2.5 \\
2.3 \\
1.1\end{array}$ \\
\hline 10 & $\begin{array}{l}08-15-83 L \\
12-21-83 \\
03-13-84 L\end{array}$ & $\begin{array}{r}327 \\
98 \\
673\end{array}$ & $\begin{array}{r}29.0 \\
24.0\end{array}$ & $\begin{array}{l}7.20 \\
6.00 \\
7.60\end{array}$ & $\begin{array}{r}75 \\
340 \\
120\end{array}$ & $\begin{array}{r}130 \\
42 \\
270\end{array}$ & $\begin{array}{l}-- \\
17 \\
16\end{array}$ & $\begin{array}{r}45 \\
14 \\
100\end{array}$ & $\begin{array}{l}3.7 \\
1.7 \\
5.2\end{array}$ & $\frac{19}{7.1}$ & $\begin{array}{r}1.4 \\
.90 \\
.90\end{array}$ \\
\hline 11 & $\begin{array}{l}08-15-83 L \\
12-21-83 \\
03-13-84 \mathrm{~L} \\
04-04-84 \\
06-30-84 \mathrm{C}\end{array}$ & $\begin{array}{l}337 \\
145 \\
588 \\
172 \\
293\end{array}$ & $\begin{array}{r}29.0 \\
- \\
23.5 \\
-- \\
--\end{array}$ & $\begin{array}{l}7.40 \\
6.50 \\
7.60 \\
6.90 \\
7.80\end{array}$ & $\begin{array}{r}120 \\
300 \\
160 \\
140 \\
80\end{array}$ & $\begin{array}{r}130 \\
57 \\
240 \\
67 \\
110\end{array}$ & $\begin{array}{l}-- \\
15 \\
22 \\
14 \\
12\end{array}$ & $\begin{array}{l}45 \\
19 \\
87 \\
24 \\
41\end{array}$ & $\begin{array}{l}4.4 \\
2.2 \\
4.8 \\
1.7 \\
2.6\end{array}$ & $\begin{array}{l}21 \\
10 \\
33 \\
11 \\
16\end{array}$ & $\begin{array}{l}1.7 \\
1.3 \\
1.0 \\
1.8 \\
1.5\end{array}$ \\
\hline 12 & $\begin{array}{l}08-15-83 L \\
12-21-83 \\
03-13-84 L \\
04-04-84 \\
08-20-84 C\end{array}$ & $\begin{array}{l}294 \\
140 \\
536 \\
155 \\
245\end{array}$ & 20.0 & $\begin{array}{l}7.10 \\
6.60 \\
7.60 \\
6.90 \\
7.80\end{array}$ & $\begin{array}{r}100 \\
280 \\
140 \\
85 \\
55\end{array}$ & $\begin{array}{r}-- \\
51 \\
220 \\
58 \\
92\end{array}$ & $\begin{array}{r}-- \\
12 \\
23 \\
10 \\
9\end{array}$ & $\begin{array}{l}-- \\
17 \\
80 \\
21 \\
33\end{array}$ & $\begin{array}{l}3.9 \\
2.1 \\
4.6 \\
1.3 \\
2.2\end{array}$ & $\begin{array}{l}19 \\
10 \\
28 \\
7.5 \\
12\end{array}$ & $\begin{array}{l}1.7 \\
1.3 \\
.90 \\
2.1 \\
1.8\end{array}$ \\
\hline
\end{tabular}


APPENDIX IV--Quality of water analyses--Continued

[L denotes low flow sample; C denotes a composite sample; $S$ denotes calculated by summing components]

\begin{tabular}{|c|c|c|c|c|c|c|c|c|c|c|c|}
\hline $\begin{array}{l}\text { Site } \\
\text { No. }\end{array}$ & $\begin{array}{c}\text { Date } \\
\text { of } \\
\text { sample }\end{array}$ & $\begin{array}{l}\text { Alka- } \\
\text { lin- } \\
\text { ity } \\
\mathrm{Lab} \\
\text { (mg/L } \\
\text { as } \\
\mathrm{CaCO}_{3} \text { ) }\end{array}$ & $\begin{array}{l}\text { Sul- } \\
\text { fate } \\
\text { dis- } \\
\text { solved } \\
\text { (mg/L } \\
\text { as } \\
\mathrm{SO}_{4} \text { ) }\end{array}$ & $\begin{array}{l}\text { Chlo- } \\
\text { ride, } \\
\text { dis- } \\
\text { solved } \\
\text { (mg/L } \\
\text { as Cl) }\end{array}$ & $\begin{array}{l}\text { Fluo- } \\
\text { ride, } \\
\text { dis- } \\
\text { solved } \\
\text { (mg/L } \\
\text { as E) }\end{array}$ & $\begin{array}{l}\text { Sil- } \\
\text { ica, } \\
\text { dis- } \\
\text { solved } \\
\text { (mg/L } \\
\text { as } \\
\mathrm{SiO}_{2} \text { ) }\end{array}$ & $\begin{array}{l}\text { Solids, } \\
\text { resi- } \\
\text { due at } \\
180{ }^{-} \mathrm{C} \\
\text { dis- } \\
\text { solved } \\
\text { (mg/L) }\end{array}$ & $\begin{array}{l}\text { Nitro- } \\
\text { gen, } \\
\text { ni- } \\
\text { trate, } \\
\text { total } \\
\text { (mg/L } \\
\text { as } N \text { ) }\end{array}$ & $\begin{array}{l}\text { Nitro- } \\
\text { gen, } \\
\text { ni- } \\
\text { trite } \\
\text { total } \\
\text { (ms/L } \\
\text { as } N \text { ) }\end{array}$ & $\begin{array}{l}\text { Nitro- } \\
\text { gen, } \\
\text { ammo- } \\
\text { nia, } \\
\text { total } \\
\text { (mg/L } \\
\text { as } N \text { ) }\end{array}$ & $\begin{array}{l}\text { Nitro- } \\
\text { gen, } \\
\text { or- } \\
\text { ganic } \\
\text { total } \\
\text { (mg/L) } \\
\text { as } N \text { ) }\end{array}$ \\
\hline 1 & $\begin{array}{l}03-09-84 L \\
09-28-84\end{array}$ & $\begin{array}{l}16 \\
14\end{array}$ & $\begin{array}{l}0.5 \\
2.3\end{array}$ & $\begin{array}{l}15 \\
14\end{array}$ & $\begin{array}{r}<0.10 \\
<.10\end{array}$ & $\begin{array}{l}1.4 \\
2.7\end{array}$ & $\begin{array}{l}63 \\
77\end{array}$ & $\overline{--}$ & $\begin{array}{l}0.020 \\
<.010\end{array}$ & $\begin{array}{l}0.290 \\
<.010\end{array}$ & 0.61 \\
\hline 2 & $\begin{array}{l}08-18-83 L \\
12-12-83 C \\
12-21-83 \\
03-15-84 L \\
09-28-84\end{array}$ & $\begin{array}{r}145 \\
75 \\
26 \\
157 \\
29\end{array}$ & $\begin{array}{l}24 \\
19 \\
23 \\
30 \\
8.8\end{array}$ & $\begin{array}{l}25 \\
15 \\
14 \\
27 \\
14\end{array}$ & $\begin{array}{r}.20 \\
.10 \\
<.10 \\
<.10 \\
<.10\end{array}$ & $\begin{array}{l}7.0 \\
4.1 \\
4.5 \\
5.3 \\
3.5\end{array}$ & $\begin{array}{r}263 \\
148 \\
136 \\
300 \\
87\end{array}$ & $\begin{array}{l}.00 \\
-- \\
-- \\
--\end{array}$ & $\begin{array}{r}.010 \\
-- \\
-0 \\
.010 \\
.010\end{array}$ & $\begin{array}{r}.020 \\
-- \\
-- \\
<.010 \\
<.010\end{array}$ & $\begin{array}{l}.46 \\
-- \\
-- \\
--\end{array}$ \\
\hline 3 & $\begin{array}{l}08-18-83 L \\
12-21-83 \\
02-22-84 \\
03-06-84 L \\
04-04-84 \\
05-23-84 C\end{array}$ & $\begin{array}{l}86 \\
16 \\
60 \\
44 \\
19 \\
19\end{array}$ & $\begin{array}{l}35 \\
6.1 \\
19 \\
40 \\
4.6 \\
7.7\end{array}$ & $\begin{array}{r}20 \\
11 \\
18 \\
26 \\
3.4 \\
4.0\end{array}$ & $\begin{array}{r}.20 \\
<.10 \\
<.10 \\
<.10 \\
<.10 \\
<.10\end{array}$ & $\begin{array}{l}6.2 \\
4.1 \\
4.9 \\
6.6 \\
1.1 \\
1.0\end{array}$ & $\begin{array}{r}200 \\
93 \\
143 \\
169 \\
47 \\
56\end{array}$ & $\begin{array}{l}-- \\
-- \\
-- \\
-- \\
--\end{array}$ & $\begin{array}{r}<.010 \\
-- \\
- \\
<.010 \\
<.010 \\
.010\end{array}$ & $\begin{array}{r}.020 \\
-- \\
-0 \\
.010 \\
.020 \\
.030\end{array}$ & $\begin{array}{c}.42 \\
-- \\
-- \\
-- \\
.98 \\
--\end{array}$ \\
\hline 4 & $\begin{array}{l}08-10-83 L \\
12-21-83 \\
02-22-84 C \\
03-06-84 L \\
09-28-84\end{array}$ & $\begin{array}{r}66 \\
37 \\
104 \\
133 \\
36\end{array}$ & $\begin{array}{l}5.8 \\
13 \\
13 \\
24 \\
4.7\end{array}$ & $\begin{array}{l}9.3 \\
11 \\
19 \\
34 \\
10\end{array}$ & $\begin{array}{r}.10 \\
<.10 \\
.20 \\
.10 \\
<.10\end{array}$ & $\begin{array}{l}2.2 \\
3.3 \\
5.3 \\
4.9 \\
3.6\end{array}$ & $\begin{array}{r}107 \\
116 \\
194 \\
261 \\
83\end{array}$ & $\begin{array}{l}.01 \\
-- \\
-- \\
--\end{array}$ & $\begin{array}{r}.010 \\
-- \\
-0 \\
<.010 \\
<.010\end{array}$ & $\begin{array}{r}.010 \\
-- \\
-0 \\
<.010 \\
<.010\end{array}$ & $\begin{array}{l}.07 \\
-- \\
-- \\
--\end{array}$ \\
\hline 5 & $\begin{array}{l}08-18-83 L \\
12-21-83 \\
03-19-84 L \\
09-28-84\end{array}$ & $\begin{array}{r}1.0 \\
<1.0 \\
<1.0 \\
<1.0\end{array}$ & $\begin{array}{l}<.1 \\
-- \\
-5\end{array}$ & $\begin{array}{l}10 \\
12 \\
13 \\
10\end{array}$ & $\begin{array}{r}.20 \\
<.10 \\
<.10 \\
<.10\end{array}$ & $\begin{array}{r}3.0 \\
2.5 \\
-- \\
2.1\end{array}$ & $\begin{array}{r}114 \\
108 \\
46 \\
80\end{array}$ & $\begin{array}{l}.00 \\
-- \\
--\end{array}$ & $\begin{array}{r}.020 \\
.010 \\
.010\end{array}$ & $\begin{array}{r}.030 \\
-- \\
.010 \\
.010\end{array}$ & $\begin{array}{r}1.1 \\
-6 \\
.69 \\
.69\end{array}$ \\
\hline 6 & $\begin{array}{l}08-10-83 L \\
12-20-83 C \\
12-21-83 \\
12-22-83 \\
02-22-84 \\
03-09-84 L\end{array}$ & $\begin{array}{r}58 \\
58 \\
46 \\
73 \\
142 \\
178\end{array}$ & $\begin{array}{l}7.4 \\
11 \\
12 \\
14 \\
10 \\
13\end{array}$ & $\begin{array}{l}17 \\
15 \\
13 \\
19 \\
31 \\
42\end{array}$ & $\begin{array}{r}.10 \\
<.10 \\
<.10 \\
<.10 \\
.20 \\
.10\end{array}$ & $\begin{array}{l}3.0 \\
3.5 \\
3.4 \\
4.6 \\
4.8 \\
4.9\end{array}$ & $\begin{array}{l}135 \\
137 \\
127 \\
171 \\
244 \\
322\end{array}$ & $\begin{array}{l}.01 \\
-- \\
-- \\
--\end{array}$ & $\begin{array}{r}.010 \\
=- \\
\overline{-} \\
-\overline{-} \\
<.010\end{array}$ & $\begin{array}{c}.020 \\
-- \\
-- \\
-- \\
.050\end{array}$ & $\begin{array}{l}.30 \\
-- \\
-- \\
\overline{--} \\
.65\end{array}$ \\
\hline 7 & $\begin{array}{l}08-10-83 L \\
12-21-83 \\
02-22-84 \\
03-19-84 L \\
04-04-84 \\
05-23-84 \mathrm{C}\end{array}$ & $\begin{array}{r}-- \\
35 \\
84 \\
168 \\
57 \\
39\end{array}$ & $\begin{array}{l}6.8 \\
11 \\
-- \\
9.0 \\
5.8 \\
5.2\end{array}$ & $\begin{array}{l}7.5 \\
8.0 \\
10 \\
31 \\
8.0 \\
8.2\end{array}$ & $\begin{array}{r}.10 \\
<.10 \\
<.10 \\
.20 \\
<.10 \\
.10\end{array}$ & $\begin{array}{l}3.6 \\
2.8 \\
2.4 \\
3.2 \\
1.7 \\
1.4\end{array}$ & $\begin{array}{r}109 \\
89 \\
122 \\
279 \\
90 \\
89\end{array}$ & $\begin{array}{c}.01 \\
-- \\
-- \\
-- \\
.17\end{array}$ & $\begin{array}{r}.010 \\
-0 \\
-0 \\
<.010 \\
<.010 \\
.030\end{array}$ & $\begin{array}{r}.020 \\
-- \\
-- \\
.050 \\
.100 \\
.190\end{array}$ & $\begin{array}{r}.05 \\
-- \\
-7 \\
.75 \\
.90 \\
1.1\end{array}$ \\
\hline 8 & $\begin{array}{l}12-21-83 \\
03-19-84 L \\
09-28-84\end{array}$ & $\begin{array}{l}-- \\
-- \\
<1.0\end{array}$ & $=-$ & $\begin{array}{l}12 \\
14 \\
12\end{array}$ & $\begin{array}{l}<.10 \\
<.10 \\
<.10\end{array}$ & $\begin{array}{r}2.3 \\
.4 \\
2.1\end{array}$ & $\begin{array}{r}106 \\
56 \\
81\end{array}$ & $\begin{array}{l}-- \\
--\end{array}$ & $\begin{array}{l}.0- \\
.020 \\
.010\end{array}$ & $\begin{array}{l}1.40^{--} \\
.020\end{array}$ & $\begin{array}{r}-- \\
0.00 \\
.78\end{array}$ \\
\hline 9 & $\begin{array}{l}08-18-83 L \\
12-21-83 \\
03-09-84 L \\
09-28-84\end{array}$ & $\begin{array}{l}-- \\
16 \\
23 \\
9.0\end{array}$ & $\begin{array}{r}18 \\
19 \\
1.6\end{array}$ & $\begin{array}{l}14 \\
12 \\
15 \\
11\end{array}$ & $\begin{array}{r}.50 \\
<.10 \\
<.10 \\
<.10\end{array}$ & $\begin{array}{l}5.0 \\
2.4 \\
1.4 \\
3.0\end{array}$ & $\begin{array}{r}152 \\
104 \\
127 \\
90\end{array}$ & $\begin{array}{l}.18 \\
-08 \\
.0\end{array}$ & $\begin{array}{r}.020 \\
.020 \\
.010\end{array}$ & $\begin{array}{r}.050 \\
-- \\
.080 \\
.050\end{array}$ & $\begin{array}{l}1.8 \\
1.0 \\
1.2\end{array}$ \\
\hline 10 & $\begin{array}{l}08-15-83 L \\
12-21-83 \\
03-13-84 L\end{array}$ & $\begin{array}{r}-- \\
25 \\
256\end{array}$ & $\begin{array}{l}17 \\
20 \\
15\end{array}$ & $\begin{array}{l}27 \\
13 \\
57\end{array}$ & $\begin{array}{r}.20 \\
<.10 \\
.20\end{array}$ & $\begin{array}{r}5.7 \\
3.2 \\
13\end{array}$ & $\begin{array}{l}215 \\
126 \\
458\end{array}$ & $\frac{.05}{.-17}$ & $\begin{array}{r}.010 \\
.030\end{array}$ & $\begin{array}{r}.160 \\
-- \\
.230\end{array}$ & $\frac{.79}{.97}$ \\
\hline 11 & $\begin{array}{l}08-15-83 L \\
12-21-83 \\
03-13-84 L \\
04-04-84 \\
06-30-84 C\end{array}$ & $\begin{array}{r}-- \\
42 \\
216 \\
53 \\
102\end{array}$ & $\begin{array}{l}15 \\
15 \\
17 \\
9.8 \\
9.9\end{array}$ & $\begin{array}{l}30 \\
15 \\
50 \\
16 \\
27\end{array}$ & $\begin{array}{r}.20 \\
<.10 \\
.10 \\
.10 \\
.20\end{array}$ & $\begin{array}{c}7.2 \\
2.9 \\
10 \\
1.8 \\
5.6\end{array}$ & $\begin{array}{l}230 \\
136 \\
420 \\
127 \\
204\end{array}$ & $\begin{array}{l}.13 \\
-- \\
.07 \\
.26 \\
.07\end{array}$ & $\begin{array}{l}.020 \\
-- \\
.030 \\
.040 \\
.030\end{array}$ & $\begin{array}{r}.100 \\
-- \\
.250 \\
.140 \\
.060\end{array}$ & $\begin{array}{l}1.9 \\
1.1 \\
1.7 \\
.74\end{array}$ \\
\hline 12 & $\begin{array}{l}08-15-83 L \\
12-21-83 \\
03-13-84 L \\
04-04-84 \\
08-20-84 C\end{array}$ & $\begin{array}{r}-- \\
39 \\
197 \\
48 \\
83\end{array}$ & $\begin{array}{r}13 \\
14 \\
15 \\
9.5 \\
8.1\end{array}$ & $\begin{array}{l}27 \\
15 \\
45 \\
13 \\
20\end{array}$ & $\begin{array}{r}.20 \\
<.10 \\
.10 \\
.10 \\
.10\end{array}$ & $\begin{array}{l}6.4 \\
2.8 \\
9.4 \\
1.6 \\
3.3\end{array}$ & $\begin{array}{l}207 \\
132 \\
378 \\
107 \\
154\end{array}$ & $\begin{array}{l}.10 \\
.07 \\
.36 \\
.17\end{array}$ & $\begin{array}{r}.020 \\
.030 \\
.040 \\
.030\end{array}$ & $\begin{array}{r}.080 \\
-170 \\
.180 \\
<.010\end{array}$ & $\begin{array}{l}2.2 \\
1.0 \\
1.6 \\
-\end{array}$ \\
\hline
\end{tabular}


APPENDIX IV--0uality of water analyses--Continued

[L denotes low flow sample; C denotes a composite sample; S denotes calculated by summing components]

\begin{tabular}{|c|c|c|c|c|c|c|c|c|c|c|c|}
\hline $\begin{array}{l}\text { Site } \\
\text { No. }\end{array}$ & $\begin{array}{l}\text { Date } \\
\text { of } \\
\text { sample }\end{array}$ & $\begin{array}{l}\text { Ni- } \\
\text { tro- } \\
\text { gen, } \\
\text { total } \\
\text { (mg/L } \\
\text { as N) }\end{array}$ & $\begin{array}{l}\text { Phos- } \\
\text { phor- } \\
\text { us, } \\
\text { total } \\
\text { (ms/L } \\
\text { as P) }\end{array}$ & $\begin{array}{l}\text { Ortho- } \\
\text { phos- } \\
\text { phorus, } \\
\text { total } \\
\text { (ms/L } \\
\text { as P) }\end{array}$ & $\begin{array}{l}\text { Alum- } \\
\text { inum, } \\
\text { total } \\
\text { recov- } \\
\text { erable } \\
(\mu B / L \\
\text { as } A 1)\end{array}$ & $\begin{array}{l}\text { Ar- } \\
\text { sen- } \\
\text { ic, } \\
\text { total } \\
\text { ( } \mu \mathrm{g} / \mathrm{L} \\
\text { as As) }\end{array}$ & $\begin{array}{l}\text { Cad- } \\
\text { mium, } \\
\text { total } \\
\text { recov- } \\
\text { erable } \\
(\mu 8 / L \\
\text { as Cd) }\end{array}$ & $\begin{array}{l}\text { Cop- } \\
\text { per, } \\
\text { dis- } \\
\text { solved } \\
(\mu g / L \\
\text { as } \mathrm{Cu})\end{array}$ & $\begin{array}{l}\text { Iron, } \\
\text { total } \\
\text { recov- } \\
\text { erable } \\
(\mu g / L \\
\text { as } F \theta)\end{array}$ & $\begin{array}{l}\text { Iron, } \\
\text { dis- } \\
\text { solved } \\
(\mu g / L \\
\text { as Fe) }\end{array}$ & $\begin{array}{l}\text { Lead, } \\
\text { total } \\
\text { recov- } \\
\text { erable } \\
\text { ( } \mu 8 / L \\
\text { as } \mathrm{Pb})\end{array}$ \\
\hline 1 & $\begin{array}{l}03-09-84 L \\
09-28-84\end{array}$ & 0.925 & $\begin{array}{r}0.050 \\
.080\end{array}$ & $\begin{array}{r}<0.010 \\
.050\end{array}$ & $\begin{array}{l}310 \\
520\end{array}$ & $\begin{array}{l}1 \\
1\end{array}$ & $\begin{array}{l}1 \\
6\end{array}$ & $\begin{array}{r}<1 \\
3\end{array}$ & $\begin{array}{l}640 \\
810\end{array}$ & $\begin{array}{l}540 \\
400\end{array}$ & $\begin{array}{l}1 \\
2\end{array}$ \\
\hline 2 & $\begin{array}{l}08-18-83 L \\
12-12-83 C \\
12-21-83 \\
03-15-84 L \\
09-28-84\end{array}$ & $\begin{array}{c}49 \\
-- \\
-- \\
-- \\
--\end{array}$ & $\begin{array}{r}.050 \\
-- \\
-- \\
.050 \\
.110\end{array}$ & $\begin{array}{r}.010 \\
-- \\
-- \\
.020 \\
.050\end{array}$ & $\begin{array}{r}230 \\
-- \\
-- \\
90 \\
790\end{array}$ & $\begin{array}{r}2 \\
-- \\
-1 \\
1\end{array}$ & $\begin{array}{r}2 \\
-- \\
--1 \\
2\end{array}$ & $\begin{array}{r}<1 \\
-- \\
-- \\
<1 \\
2\end{array}$ & $\begin{array}{r}290 \\
-- \\
-\overline{-} \\
310 \\
660\end{array}$ & $\begin{array}{r}130 \\
-- \\
-- \\
160 \\
240\end{array}$ & $\begin{array}{r}4 \\
-- \\
-1 \\
5\end{array}$ \\
\hline 3 & $\begin{array}{l}08-18-83 L \\
12-21-83 \\
02-22-84 \\
03-06-84 L \\
04-04-84 \\
05-23-84 C\end{array}$ & $\begin{array}{c}.45 \\
-- \\
-- \\
-- \\
1.0 \mathrm{~s} \\
--\end{array}$ & $\begin{array}{r}.010 \\
-- \\
-- \\
<.010 \\
.030 \\
.060\end{array}$ & $\begin{array}{r}<.010 \\
-- \\
-- \\
<.010 \\
.010 \\
.020\end{array}$ & $\begin{array}{r}-- \\
-- \\
190 \\
640 \\
8200\end{array}$ & $\begin{array}{r}-- \\
-- \\
-1 \\
1 \\
<1\end{array}$ & $\begin{array}{r}-- \\
-- \\
-- \\
<1 \\
1\end{array}$ & $\begin{array}{l}-- \\
-- \\
<1 \\
<1 \\
4\end{array}$ & $\begin{array}{r}-- \\
-- \\
750 \\
850 \\
1100\end{array}$ & $\begin{array}{r}-- \\
-- \\
-- \\
410 \\
85 \\
110\end{array}$ & $\begin{array}{r}-- \\
-- \\
-- \\
<1 \\
4 \\
2\end{array}$ \\
\hline 4 & $\begin{array}{l}08-10-83 L \\
12-21-83 \\
02-22-84 \mathrm{C} \\
03-06-84 \mathrm{~L} \\
09-28-84\end{array}$ & $\begin{array}{c}10 \\
-- \\
-- \\
-- \\
--\end{array}$ & $\begin{array}{r}.020 \\
-- \\
-- \\
<.010 \\
.040\end{array}$ & $\begin{array}{r}.010 \\
-- \\
-- \\
<.010 \\
.020\end{array}$ & $\begin{array}{r}-- \\
-- \\
-- \\
170 \\
310\end{array}$ & $\begin{array}{l}-- \\
-- \\
-1 \\
<1\end{array}$ & $\begin{array}{r}-- \\
-- \\
2 \\
1\end{array}$ & $\begin{array}{r}-- \\
-- \\
-- \\
<1\end{array}$ & $\begin{array}{r}-- \\
-- \\
1400 \\
610\end{array}$ & $\begin{array}{r}-- \\
-- \\
550 \\
280\end{array}$ & $\begin{array}{c}-- \\
-- \\
-- \\
2 \\
3\end{array}$ \\
\hline 5 & $\begin{array}{l}08-18-83 L \\
12-21-83 \\
03-19-84 L \\
09-28-84\end{array}$ & $\begin{array}{l}1.1 \\
=715 \\
.715\end{array}$ & $\begin{array}{r}.030 \\
-- \\
<.010 \\
<.010\end{array}$ & $\begin{array}{r}.010 \\
-0 \\
<.010 \\
<.010\end{array}$ & $\begin{array}{r}-- \\
270 \\
300\end{array}$ & $\begin{array}{r}-- \\
-1 \\
<1\end{array}$ & $\begin{array}{l}-- \\
<1 \\
<1\end{array}$ & $\begin{array}{r}-- \\
-- \\
6\end{array}$ & $\begin{array}{l}-- \\
-- \\
310 \\
500\end{array}$ & $\begin{array}{r}-- \\
-- \\
220 \\
390\end{array}$ & $\begin{array}{r}-- \\
-- \\
2 \\
3\end{array}$ \\
\hline 6 & $\begin{array}{l}08-10-83 L \\
12-20-83 C \\
12-21-83 \\
12-22-83 \\
02-22-84 \\
03-09-84 L\end{array}$ & $\begin{array}{c}.34 \\
-- \\
-- \\
-- \\
-- \\
.715\end{array}$ & $\begin{array}{r}.060 \\
-- \\
-- \\
-- \\
-- \\
.030\end{array}$ & $\begin{array}{r}.030 \\
-- \\
-- \\
-- \\
<.010\end{array}$ & $\begin{array}{l}-- \\
-- \\
-- \\
-- \\
-\overline{30}\end{array}$ & $\begin{array}{l}-- \\
-- \\
-- \\
-- \\
1\end{array}$ & $\begin{array}{l}-- \\
-- \\
-- \\
-- \\
-- \\
2\end{array}$ & $\begin{array}{l}-- \\
-- \\
-- \\
-- \\
--\end{array}$ & $\begin{array}{r}-- \\
-- \\
-- \\
-- \\
1800\end{array}$ & $\begin{array}{l}-- \\
-- \\
-- \\
-- \\
750\end{array}$ & $\begin{array}{l}-- \\
-- \\
-- \\
-- \\
-- \\
1\end{array}$ \\
\hline 7 & $\begin{array}{l}08-10-83 L \\
12-21-83 \\
02-22-84 \\
03-19-84 L \\
04-04-84 \\
05-23-84 C\end{array}$ & $\begin{array}{l}.09 \\
-- \\
-. \\
.815 \\
1.1 \\
1.5\end{array}$ & $\begin{array}{r}.040 \\
-- \\
-- \\
.040 \\
.030 \\
.200\end{array}$ & $\begin{array}{r}.020 \\
-- \\
-- \\
<.010 \\
.030 \\
.120\end{array}$ & $\begin{array}{r}-- \\
-- \\
\overline{60} \\
840 \\
1400\end{array}$ & $\begin{array}{l}-- \\
-- \\
1 \\
1 \\
1\end{array}$ & $\begin{array}{r}-- \\
-- \\
-- \\
1 \\
<1 \\
1\end{array}$ & $\begin{array}{r}-- \\
-- \\
-- \\
<1 \\
1 \\
5\end{array}$ & $\begin{array}{r}-- \\
-- \\
1100 \\
1300 \\
2400\end{array}$ & $\begin{array}{r}-- \\
-- \\
-- \\
360 \\
120 \\
160\end{array}$ & $\begin{array}{r}-- \\
-- \\
-- \\
2 \\
8 \\
5\end{array}$ \\
\hline 8 & $\begin{array}{l}12-21-83 \\
03-19-84 \mathrm{~L} \\
09-28-84\end{array}$ & $\begin{array}{l}-- \\
-- \\
.815\end{array}$ & $\begin{array}{r}-- \\
.010 \\
.060\end{array}$ & $\begin{array}{l}.0 \\
.010 \\
.050\end{array}$ & $\begin{array}{r}-- \\
310 \\
280\end{array}$ & $\begin{array}{l}-\frac{1}{<} \\
<1\end{array}$ & $\begin{array}{r}-- \\
1\end{array}$ & $\begin{array}{r}-- \\
2\end{array}$ & $\begin{array}{l}-- \\
460 \\
600\end{array}$ & $\begin{array}{r}-- \\
390 \\
470\end{array}$ & $\begin{array}{r}-- \\
3\end{array}$ \\
\hline 9 & $\begin{array}{l}08-18-83 L \\
12-21-83 \\
03-09-84 L \\
09-28-84\end{array}$ & $\begin{array}{l}2.0 \\
1.2 \\
1.3 \mathrm{~s}\end{array}$ & $\begin{array}{r}.520 \\
-- \\
.260 \\
.060\end{array}$ & $\begin{array}{r}.440 \\
-- \\
.210 \\
.030\end{array}$ & $\begin{array}{r}-- \\
-- \\
250 \\
610\end{array}$ & -- & $\frac{--}{2}$ & $\begin{array}{r}-- \\
-- \\
4\end{array}$ & $\begin{array}{r}-- \\
770 \\
720\end{array}$ & $\begin{array}{r}-- \\
650 \\
460\end{array}$ & $\begin{array}{r}-- \\
-- \\
3\end{array}$ \\
\hline 10 & $\begin{array}{l}08-15-83 L \\
12-21-83 \\
03-13-84 L\end{array}$ & 1.0 & $\begin{array}{r}.120 \\
.180\end{array}$ & $\begin{array}{r}.060 \\
.140\end{array}$ & $\begin{array}{r}-- \\
-- \\
120\end{array}$ & -- & -- & $\begin{array}{l}-- \\
-- \\
<1\end{array}$ & 1300 & $\begin{array}{r}-- \\
-- \\
480\end{array}$ & $\frac{--}{1}$ \\
\hline 11 & $\begin{array}{l}08-15-83 L \\
12-21-83 \\
03-13-84 L \\
04-04-84 \\
06-30-84 C\end{array}$ & $\begin{array}{l}2.2 \\
1.4 \\
2.4 \\
2.1 \\
.90\end{array}$ & $\begin{array}{r}.360 \\
-0 \\
.160 \\
.210 \\
.040\end{array}$ & $\begin{array}{r}.070 \\
-. \\
.130 \\
.190 \\
.050\end{array}$ & $\begin{array}{r}-- \\
-- \\
340 \\
30 \\
830\end{array}$ & $\begin{array}{r}-- \\
-- \\
1 \\
1\end{array}$ & $\begin{array}{r}-- \\
-- \\
<1 \\
<1\end{array}$ & $\begin{array}{r}-- \\
-- \\
<1 \\
10 \\
7\end{array}$ & $\begin{array}{r}-- \\
1100 \\
2100 \\
750\end{array}$ & $\begin{array}{r}-- \\
-- \\
430 \\
430 \\
160\end{array}$ & $\begin{array}{r}-- \\
-- \\
1 \\
2 \\
2\end{array}$ \\
\hline 12 & $\begin{array}{l}08-15-83 L \\
12-21-83 \\
03-13-84 L \\
04-04-84 \\
08-20-84 C\end{array}$ & $\begin{array}{l}2.4 \\
-- \\
1.3 \\
2.2 \\
.80\end{array}$ & $\begin{array}{r}.420 \\
-- \\
.150 \\
.190 \\
.080\end{array}$ & $\begin{array}{r}.070 \\
-- \\
.100 \\
.160 \\
.060\end{array}$ & $\begin{array}{r}1600 \\
-- \\
260 \\
4100 \\
480\end{array}$ & $\begin{array}{r}3 \\
-- \\
1 \\
2\end{array}$ & $\begin{array}{r}2 \\
--1 \\
<1 \\
2\end{array}$ & $\begin{array}{r}5 \\
--1 \\
1 \\
2\end{array}$ & $\begin{array}{r}1800 \\
-- \\
1000 \\
1600 \\
580\end{array}$ & $\begin{array}{r}320 \\
-\overline{4} \\
130 \\
90\end{array}$ & $\begin{array}{r}6 \\
--1 \\
6 \\
2\end{array}$ \\
\hline
\end{tabular}


APPENDIX IV--Quality of water analyses--Continued

[L denotes low flow sample; C denotes a composite sample; $S$ denotes calculated by summing components]

\begin{tabular}{|c|c|c|c|c|c|c|c|c|c|c|c|c|}
\hline $\begin{array}{l}\text { Site } \\
\text { No. }\end{array}$ & $\begin{array}{l}\text { Date } \\
\text { of } \\
\text { sample }\end{array}$ & $\begin{array}{l}\text { Lead, } \\
\text { dis- } \\
\text { solved } \\
(\mu 8 / L \\
\text { as } \mathrm{Pb})\end{array}$ & $\begin{array}{l}\text { Manga- } \\
\text { nese; } \\
\text { totai } \\
\text { recov- } \\
\text { erable } \\
(\mu g / L \\
\text { as } / n)\end{array}$ & $\begin{array}{l}\text { Manga- } \\
\text { nese, } \\
\text { dis- } \\
\text { solved } \\
(\mu g / L \\
\text { as } \mathrm{Mn})\end{array}$ & $\begin{array}{l}\text { Mer- } \\
\text { cury, } \\
\text { total } \\
\text { recov- } \\
\text { erable } \\
\text { ( } \mu 8 / \mathrm{L} \\
\text { as } \mathrm{Hg})\end{array}$ & $\begin{array}{l}\text { Nickel, } \\
\text { total } \\
\text { recov- } \\
\text { erable } \\
(\mu g / L \\
\text { as } N i)\end{array}$ & $\begin{array}{l}\text { Stron- } \\
\text { tium, } \\
\text { dis- } \\
\text { solved } \\
(\mu g / L \\
\text { as } S r)\end{array}$ & $\begin{array}{l}\text { Zinc, } \\
\text { dis- } \\
\text { solved } \\
(\mu g / L \\
\text { as } \mathrm{Zn})\end{array}$ & $\begin{array}{l}\text { DDD, } \\
\text { total } \\
\text { in bot- } \\
\text { tom ma- } \\
\text { terial } \\
\left(\mu 8 / K_{8}\right)\end{array}$ & $\begin{array}{l}\text { Dia- } \\
\text { zinon, } \\
\text { total } \\
(\mu \mathrm{L} / \mathrm{L})\end{array}$ & $\begin{array}{l}\text { Bepta- } \\
\text { chlor, } \\
\text { total } \\
\text { in bot- } \\
\text { tom ma- } \\
\text { terial } \\
(\mu \mathrm{g} / \mathrm{kg})\end{array}$ & $\begin{array}{l}2,4-D, \\
\text { total } \\
(\mu g / L)\end{array}$ \\
\hline 1 & $\begin{array}{l}03-09-84 L \\
09-28-84\end{array}$ & $\begin{array}{l}1 \\
6\end{array}$ & $\begin{array}{r}<10 \\
50\end{array}$ & $\begin{array}{l}3 \\
4\end{array}$ & $\begin{array}{r}0.10 \\
.20\end{array}$ & $\begin{array}{l}1 \\
1\end{array}$ & $\begin{array}{l}43 \\
36\end{array}$ & $\begin{array}{r}18 \\
8\end{array}$ & -- & $=-$ & $\overline{--}$ & $=-$ \\
\hline 2 & $\begin{array}{l}08-18-83 L \\
12-12-83 C \\
12-21-83 \\
03-15-84 L \\
08-28-84\end{array}$ & $\begin{array}{c}4 \\
-- \\
-2 \\
1 \\
5\end{array}$ & $\begin{array}{r}10 \\
-- \\
-- \\
<10 \\
40\end{array}$ & $\begin{array}{l}20 \\
-- \\
-- \\
4 \\
5\end{array}$ & $\begin{array}{r}<.10 \\
-- \\
-- \\
.10 \\
.30\end{array}$ & $\begin{array}{l}<1 \\
-- \\
-- \\
<1 \\
1\end{array}$ & $\begin{array}{r}320 \\
170 \\
76 \\
380 \\
69\end{array}$ & $\begin{array}{l}60 \\
-- \\
-- \\
11 \\
11\end{array}$ & $\begin{array}{l}.3 \\
-- \\
-- \\
--\end{array}$ & $\begin{array}{l}.01 \\
-- \\
-- \\
-- \\
--\end{array}$ & $\begin{array}{l}.2 \\
--- \\
-- \\
--\end{array}$ & $\begin{array}{c}<.01 \\
-- \\
-- \\
--\end{array}$ \\
\hline 3 & $\begin{array}{l}08-18-83 L \\
12-21-83 \\
02-22-84 \\
03-06-84 L \\
04-04-84 \\
05-23-84 C\end{array}$ & $\begin{array}{r}-- \\
-- \\
-- \\
2 \\
2 \\
3\end{array}$ & $\begin{array}{l}-- \\
-- \\
-- \\
10 \\
10 \\
20\end{array}$ & $\begin{array}{r}-- \\
-- \\
10 \\
2 \\
<1\end{array}$ & $\begin{array}{r}-- \\
-- \\
-- \\
<.10 \\
<.10 \\
.50\end{array}$ & $\begin{array}{r}-- \\
-- \\
-- \\
1 \\
2 \\
3\end{array}$ & $\begin{array}{r}180 \\
34 \\
120 \\
120 \\
34 \\
38\end{array}$ & $\begin{array}{r}-- \\
-- \\
-- \\
15 \\
37\end{array}$ & $\begin{array}{l}-- \\
-- \\
-- \\
-- \\
--\end{array}$ & $\begin{array}{l}-- \\
-- \\
-- \\
-- \\
--\end{array}$ & $\begin{array}{l}-- \\
-- \\
=- \\
-- \\
--\end{array}$ & $\begin{array}{l}-- \\
-- \\
-- \\
-- \\
--\end{array}$ \\
\hline 4 & $\begin{array}{l}08-10-83 L \\
12-21-83 \\
02-22-84 C \\
03-06-84 L \\
09-28-84\end{array}$ & $\begin{array}{r}-- \\
-- \\
-- \\
2 \\
6\end{array}$ & $\begin{array}{l}-- \\
-- \\
\overline{10} \\
10\end{array}$ & $\begin{array}{c}-- \\
-- \\
-- \\
7 \\
3\end{array}$ & $\begin{array}{r}-- \\
-- \\
-\overline{10} \\
.20\end{array}$ & $\begin{array}{l}-- \\
-- \\
-- \\
2 \\
<1\end{array}$ & $\begin{array}{r}150 \\
67 \\
190 \\
260 \\
66\end{array}$ & $\begin{array}{l}-- \\
-- \\
-- \\
17 \\
12\end{array}$ & $\begin{array}{l}-- \\
\overline{--} \\
\overline{--}\end{array}$ & $\begin{array}{l}-- \\
-- \\
-- \\
--\end{array}$ & $\begin{array}{l}-- \\
-- \\
-- \\
--\end{array}$ & $\begin{array}{l}-- \\
-- \\
-- \\
--\end{array}$ \\
\hline 5 & $\begin{array}{l}08-18-83 L \\
12-21-83 \\
03-19-84 L \\
09-28-84\end{array}$ & $\begin{array}{c}-- \\
-- \\
1 \\
6\end{array}$ & $\begin{array}{r}-- \\
-- \\
<10\end{array}$ & -- & $\begin{array}{r}-- \\
-\overline{-} \\
<.10 \\
<.10\end{array}$ & $\begin{array}{c}-- \\
-1 \\
3\end{array}$ & $\begin{array}{l}10 \\
12 \\
13 \\
12\end{array}$ & $\begin{array}{r}-- \\
-- \\
32\end{array}$ & $\begin{array}{l}-- \\
-- \\
--\end{array}$ & $\begin{array}{l}-- \\
-- \\
--\end{array}$ & $\begin{array}{l}-- \\
-- \\
-- \\
--\end{array}$ & $\begin{array}{l}-- \\
-- \\
-- \\
--\end{array}$ \\
\hline 6 & $\begin{array}{l}08-10-83 L \\
12-20-83 C \\
12-21-83 \\
12-22-83 \\
02-22-84 \\
03-09-84 L\end{array}$ & $\begin{array}{l}-- \\
-- \\
-- \\
-- \\
<1\end{array}$ & $\begin{array}{l}-- \\
=- \\
=- \\
-- \\
10\end{array}$ & $\begin{array}{l}-- \\
-- \\
-- \\
-- \\
4\end{array}$ & $\begin{array}{c}-- \\
-- \\
-- \\
-- \\
\overline{-} \\
<.10\end{array}$ & $\begin{array}{l}-- \\
-- \\
-- \\
-- \\
-- \\
1\end{array}$ & $\begin{array}{r}150 \\
94 \\
75 \\
130 \\
250 \\
330\end{array}$ & $\begin{array}{l}-- \\
-- \\
-- \\
-- \\
-- \\
12\end{array}$ & $\begin{array}{l}-- \\
-- \\
-- \\
-- \\
--\end{array}$ & $\begin{array}{l}-- \\
-- \\
-- \\
-- \\
--\end{array}$ & $\begin{array}{l}-- \\
-- \\
-- \\
-- \\
--\end{array}$ & $\begin{array}{l}-- \\
-- \\
-- \\
-- \\
-- \\
--\end{array}$ \\
\hline 7 & $\begin{array}{l}08-10-83 L \\
12-21-83 \\
02-22-84 \\
03-19-84 L \\
04-04-84 \\
05-23-84 C\end{array}$ & $\begin{array}{c}-- \\
-- \\
-- \\
2 \\
1 \\
2\end{array}$ & $\begin{array}{l}-- \\
-- \\
-- \\
10 \\
20 \\
40\end{array}$ & $\begin{array}{r}-- \\
-- \\
13 \\
2 \\
<1\end{array}$ & $\begin{array}{r}-- \\
-- \\
-- \\
.20 \\
<.10 \\
.40\end{array}$ & $\begin{array}{c}-- \\
-- \\
-- \\
1 \\
2 \\
3\end{array}$ & $\begin{array}{r}160 \\
67 \\
130 \\
340 \\
110 \\
73\end{array}$ & $\begin{array}{r}-- \\
-- \\
-- \\
5 \\
7 \\
34\end{array}$ & $\begin{array}{l}-- \\
-- \\
-- \\
-- \\
--\end{array}$ & $\begin{array}{l}-- \\
-- \\
=- \\
-- \\
--\end{array}$ & $\begin{array}{l}-- \\
-- \\
-- \\
-- \\
--\end{array}$ & $\begin{array}{l}-- \\
-- \\
-- \\
-- \\
--\end{array}$ \\
\hline 8 & $\begin{array}{l}12-21-83 \\
03-19-84 L \\
09-28-84\end{array}$ & $\begin{array}{r}-- \\
2 \\
10\end{array}$ & $\begin{array}{r}-- \\
<10 \\
20\end{array}$ & $\begin{array}{r}-- \\
12\end{array}$ & $\begin{array}{r}-- \\
<.10 \\
.20\end{array}$ & $\begin{array}{r}-- \\
<1\end{array}$ & $\begin{array}{l}13 \\
20 \\
14\end{array}$ & $\begin{array}{l}-- \\
\overline{16}\end{array}$ & $\begin{array}{l}-- \\
--\end{array}$ & $\begin{array}{l}-- \\
--\end{array}$ & $\begin{array}{l}-- \\
-- \\
--\end{array}$ & $\begin{array}{l}-- \\
--\end{array}$ \\
\hline $\boldsymbol{9}$ & $\begin{array}{l}08-18-83 L \\
12-21-83 \\
03-09-84 L \\
09-28-84\end{array}$ & $\begin{array}{c}-- \\
-- \\
1 \\
6\end{array}$ & $\begin{array}{l}-- \\
\overline{10} \\
20\end{array}$ & $\begin{array}{r}-- \\
-5 \\
7\end{array}$ & $\begin{array}{r}-- \\
-- \\
\times 10 \\
.10\end{array}$ & $\begin{array}{r}-- \\
-- \\
<1 \\
2\end{array}$ & $\begin{array}{r}110 \\
29 \\
45 \\
25\end{array}$ & $\begin{array}{l}-- \\
-- \\
18 \\
27\end{array}$ & $\begin{array}{l}-- \\
-- \\
--\end{array}$ & $\begin{array}{l}-- \\
-- \\
--\end{array}$ & $\begin{array}{l}-- \\
-- \\
--\end{array}$ & $\begin{array}{l}-- \\
-- \\
--\end{array}$ \\
\hline 10 & $\begin{array}{l}08-15-83 L \\
12-21-83 \\
03-13-84 L\end{array}$ & $\begin{array}{l}-- \\
--\end{array}$ & $\overline{--}$ & $\overline{--}$ & $\overline{.-}$ & -- & $\begin{array}{r}280 \\
67 \\
620\end{array}$ & $\overline{--}$ & $\begin{array}{l}-- \\
--\end{array}$ & $\begin{array}{l}-- \\
--\end{array}$ & $\begin{array}{l}-- \\
--\end{array}$ & $\begin{array}{l}-- \\
--\end{array}$ \\
\hline 11 & $\begin{array}{l}08-15-83 L \\
12-21-83 \\
03-13-84 \mathrm{~L} \\
04-04-84 \\
06-30-84 \mathrm{C}\end{array}$ & $\begin{array}{r}-- \\
-- \\
<1 \\
3 \\
1\end{array}$ & $\begin{array}{l}-- \\
-- \\
20 \\
20 \\
10\end{array}$ & $\begin{array}{r}-- \\
-- \\
18 \\
<10 \\
<1\end{array}$ & $\begin{array}{l}-- \\
-. \\
.20 \\
.10 \\
.30\end{array}$ & $\begin{array}{r}-- \\
-- \\
<1 \\
2 \\
1\end{array}$ & $\begin{array}{r}240 \\
94 \\
520 \\
130 \\
230\end{array}$ & $\begin{array}{r}-- \\
-- \\
8 \\
7 \\
13\end{array}$ & $\begin{array}{l}-- \\
-- \\
-- \\
--\end{array}$ & $\begin{array}{l}-- \\
-- \\
-- \\
--\end{array}$ & $\begin{array}{l}-- \\
-- \\
-- \\
--\end{array}$ & $\begin{array}{l}-- \\
-- \\
-- \\
--\end{array}$ \\
\hline 12 & $\begin{array}{l}08-15-83 L \\
12-21-83 \\
03-13-84 L \\
04-04-84 \\
08-20-84 C\end{array}$ & $\begin{array}{r}<1 \\
-- \\
<1 \\
2 \\
2\end{array}$ & $\begin{array}{r}20 \\
-- \\
20 \\
10 \\
<10\end{array}$ & $\begin{array}{r}10 \\
-- \\
16 \\
1 \\
<10\end{array}$ & $\begin{array}{r}<.10 \\
-- \\
<.10 \\
.10 \\
.30\end{array}$ & $\begin{array}{r}4 \\
-- \\
1 \\
3 \\
3\end{array}$ & $\begin{array}{r}240 \\
87 \\
470 \\
100 \\
200\end{array}$ & $\begin{array}{l}20 \\
\overline{11} \\
7 \\
10\end{array}$ & $\begin{array}{l}<.1 \\
-- \\
-- \\
--\end{array}$ & $\begin{array}{l}.23 \\
-- \\
-- \\
-- \\
--\end{array}$ & $\begin{array}{l}<.1 \\
-- \\
-- \\
--\end{array}$ & $\begin{array}{l}.09 \\
--- \\
-- \\
--\end{array}$ \\
\hline
\end{tabular}


Appendix V 


\begin{tabular}{|c|c|c|c|c|c|c|c|c|c|c|c|c|}
\hline Day & Oct & Alov & Dec & $\operatorname{Jan}$ & Fob & Mar & Apr & May & June & July & Aus & Sept \\
\hline & \multicolumn{12}{|c|}{$\begin{array}{l}\text { Site 1--Tomoka River near Bolly Bill } \\
\text { [Water year October } 1982 \text { to September 1983] }\end{array}$} \\
\hline $\begin{array}{l}1 \\
2 \\
3 \\
4 \\
5\end{array}$ & $\begin{array}{l}--- \\
--- \\
--- \\
--- \\
---\end{array}$ & $\begin{array}{l}--- \\
--- \\
--- \\
---\end{array}$ & $\begin{array}{l}--- \\
--- \\
--- \\
---\end{array}$ & $\begin{array}{l}--- \\
--- \\
--- \\
---\end{array}$ & $\begin{array}{l}--- \\
--- \\
--- \\
---\end{array}$ & $\begin{array}{l}16.1 \\
15.9 \\
17.1 \\
17.4 \\
18.5\end{array}$ & $\begin{array}{l}17.0 \\
17.4 \\
17.7 \\
17.1 \\
18.8\end{array}$ & $\begin{array}{l}20.2 \\
20.4 \\
21.7 \\
20.8 \\
19.1\end{array}$ & $\begin{array}{l}24.2 \\
25.0 \\
25.8 \\
26.1 \\
24.5\end{array}$ & $\begin{array}{l}25.7 \\
25.6 \\
25.7 \\
25.9 \\
25.8\end{array}$ & $\begin{array}{l}--- \\
--- \\
--- \\
--- \\
---\end{array}$ & $\begin{array}{l}25.7 \\
25.2 \\
25.1 \\
26.2 \\
27.1\end{array}$ \\
\hline $\begin{array}{r}6 \\
7 \\
8 \\
9 \\
10\end{array}$ & $\begin{array}{l}--- \\
--- \\
--- \\
--- \\
---\end{array}$ & $\begin{array}{l}--- \\
--- \\
--- \\
---\end{array}$ & $\begin{array}{l}--- \\
--- \\
--- \\
---\end{array}$ & $\begin{array}{l}--- \\
--- \\
--- \\
---\end{array}$ & $\begin{array}{l}--- \\
--- \\
-- \\
-- \\
---\end{array}$ & $\begin{array}{l}20.7 \\
20.3 \\
19.3 \\
19.0 \\
16.8\end{array}$ & $\begin{array}{l}20.4 \\
21.2 \\
21.8 \\
22.0 \\
21.0\end{array}$ & $\begin{array}{l}18.9 \\
19.3 \\
19.7 \\
20.5 \\
21.2\end{array}$ & $\begin{array}{l}24.4 \\
24.1 \\
23.8 \\
24.1 \\
24.3\end{array}$ & $\begin{array}{l}25.9 \\
26.1 \\
25.8 \\
26.1 \\
25.8\end{array}$ & $\begin{array}{r}--- \\
--- \\
26.6 \\
26.1 \\
25.4\end{array}$ & $\begin{array}{r}27.0 \\
26.2 \\
- \\
- \\
- \\
--\end{array}$ \\
\hline $\begin{array}{l}11 \\
12 \\
13 \\
14 \\
15\end{array}$ & $\begin{array}{l}--- \\
--- \\
--- \\
--- \\
---\end{array}$ & $\begin{array}{l}--- \\
--- \\
--- \\
---\end{array}$ & $\begin{array}{l}--- \\
--- \\
--- \\
---\end{array}$ & $\begin{array}{l}--- \\
--- \\
--- \\
--- \\
--\end{array}$ & $\begin{array}{l}15.5 \\
14.1 \\
14.5 \\
13.3 \\
13.1\end{array}$ & $\begin{array}{l}13.2 \\
12.6 \\
12.6 \\
13.2 \\
14.9\end{array}$ & $\begin{array}{l}19.6 \\
19.2 \\
19.1 \\
19.9 \\
20.4\end{array}$ & $\begin{array}{l}20.9 \\
21.3 \\
21.3 \\
21.1 \\
21.8\end{array}$ & $\begin{array}{l}24.7 \\
25.2 \\
24.3 \\
24.2 \\
24.3\end{array}$ & $\begin{array}{l}25.4 \\
26.1 \\
26.2 \\
26.5 \\
27.2\end{array}$ & $\begin{array}{l}26.5 \\
26.2 \\
25.2 \\
24.8 \\
24.8\end{array}$ & $\begin{array}{l}--- \\
--- \\
--- \\
--- \\
--\end{array}$ \\
\hline $\begin{array}{l}16 \\
17 \\
18 \\
19 \\
20\end{array}$ & $\begin{array}{l}-- \\
--- \\
--- \\
--- \\
---\end{array}$ & $\begin{array}{l}--- \\
--- \\
--- \\
--- \\
--\end{array}$ & $\begin{array}{l}--- \\
--- \\
--- \\
---\end{array}$ & $\begin{array}{l}--- \\
--- \\
--- \\
---\end{array}$ & $\begin{array}{l}13.7 \\
14.7 \\
14.3 \\
14.3 \\
15.4\end{array}$ & $\begin{array}{l}17.3 \\
18.6 \\
18.3 \\
18.0 \\
18.1\end{array}$ & $\begin{array}{l}19.2 \\
17.6 \\
16.8 \\
16.9 \\
16.4\end{array}$ & $\begin{array}{l}22.8 \\
23.8 \\
23.9 \\
23.8 \\
24.0\end{array}$ & $\begin{array}{l}24.1 \\
24.6 \\
24.2 \\
24.6 \\
24.9\end{array}$ & $\begin{array}{l}27.8 \\
28.2 \\
28.3 \\
27.5 \\
26.3\end{array}$ & $\begin{array}{l}25.1 \\
25.4 \\
25.3 \\
25.7 \\
26.1\end{array}$ & $\begin{array}{l}--- \\
--- \\
--- \\
--- \\
--\end{array}$ \\
\hline $\begin{array}{l}21 \\
22 \\
23 \\
24 \\
25\end{array}$ & $\begin{array}{l}--- \\
--- \\
--- \\
--- \\
---\end{array}$ & $\begin{array}{l}--- \\
--- \\
--- \\
---\end{array}$ & $\begin{array}{l}--- \\
--- \\
--- \\
--- \\
---\end{array}$ & $\begin{array}{l}--- \\
--- \\
--- \\
--- \\
---\end{array}$ & $\begin{array}{l}16.3 \\
17.3 \\
17.9 \\
16.9 \\
16.7\end{array}$ & $\begin{array}{l}18.9 \\
16.3 \\
15.1 \\
16.3 \\
14.6\end{array}$ & $\begin{array}{l}16.2 \\
16.9 \\
18.8 \\
19.1 \\
18.3\end{array}$ & $\begin{array}{l}24.7 \\
24.9 \\
25.3 \\
25.9 \\
25.6\end{array}$ & $\begin{array}{l}25.3 \\
24.9 \\
24.7 \\
24.5 \\
24.6\end{array}$ & $\begin{array}{l}--- \\
--- \\
--- \\
---\end{array}$ & $\begin{array}{l}26.5 \\
26.7 \\
26.4 \\
26.8 \\
26.8\end{array}$ & $\begin{array}{l}--- \\
--- \\
--- \\
--- \\
--\end{array}$ \\
\hline $\begin{array}{l}26 \\
27 \\
28 \\
29 \\
30 \\
31\end{array}$ & $\begin{array}{l}--- \\
--- \\
--- \\
--- \\
--- \\
---\end{array}$ & $\begin{array}{l}--- \\
--- \\
--- \\
--- \\
---\end{array}$ & $\begin{array}{l}--- \\
--- \\
--- \\
--- \\
---\end{array}$ & $\begin{array}{l}--- \\
--- \\
--- \\
--- \\
---\end{array}$ & $\begin{array}{r}14.4 \\
14.2 \\
15.9 \\
-.- \\
-.- \\
---\end{array}$ & $\begin{array}{l}14.5 \\
16.1 \\
17.4 \\
16.3 \\
15.8 \\
17.3\end{array}$ & $\begin{array}{r}17.7 \\
17.7 \\
18.2 \\
18.6 \\
19.1 \\
-\end{array}$ & $\begin{array}{l}25.1 \\
24.9 \\
25.3 \\
24.8 \\
22.9 \\
22.6\end{array}$ & $\begin{array}{r}25.3 \\
25.6 \\
25.2 \\
25.7 \\
25.8 \\
-.-\end{array}$ & $\begin{array}{l}--- \\
--- \\
--- \\
--- \\
--- \\
---\end{array}$ & $\begin{array}{l}26.6 \\
26.4 \\
26.2 \\
26.7 \\
26.9 \\
26.2\end{array}$ & $\begin{array}{l}--- \\
--- \\
--- \\
--- \\
--- \\
---\end{array}$ \\
\hline
\end{tabular}

Site 1--Tomoka River near Bolly Bill

[Water year October 1983 to September 1984]

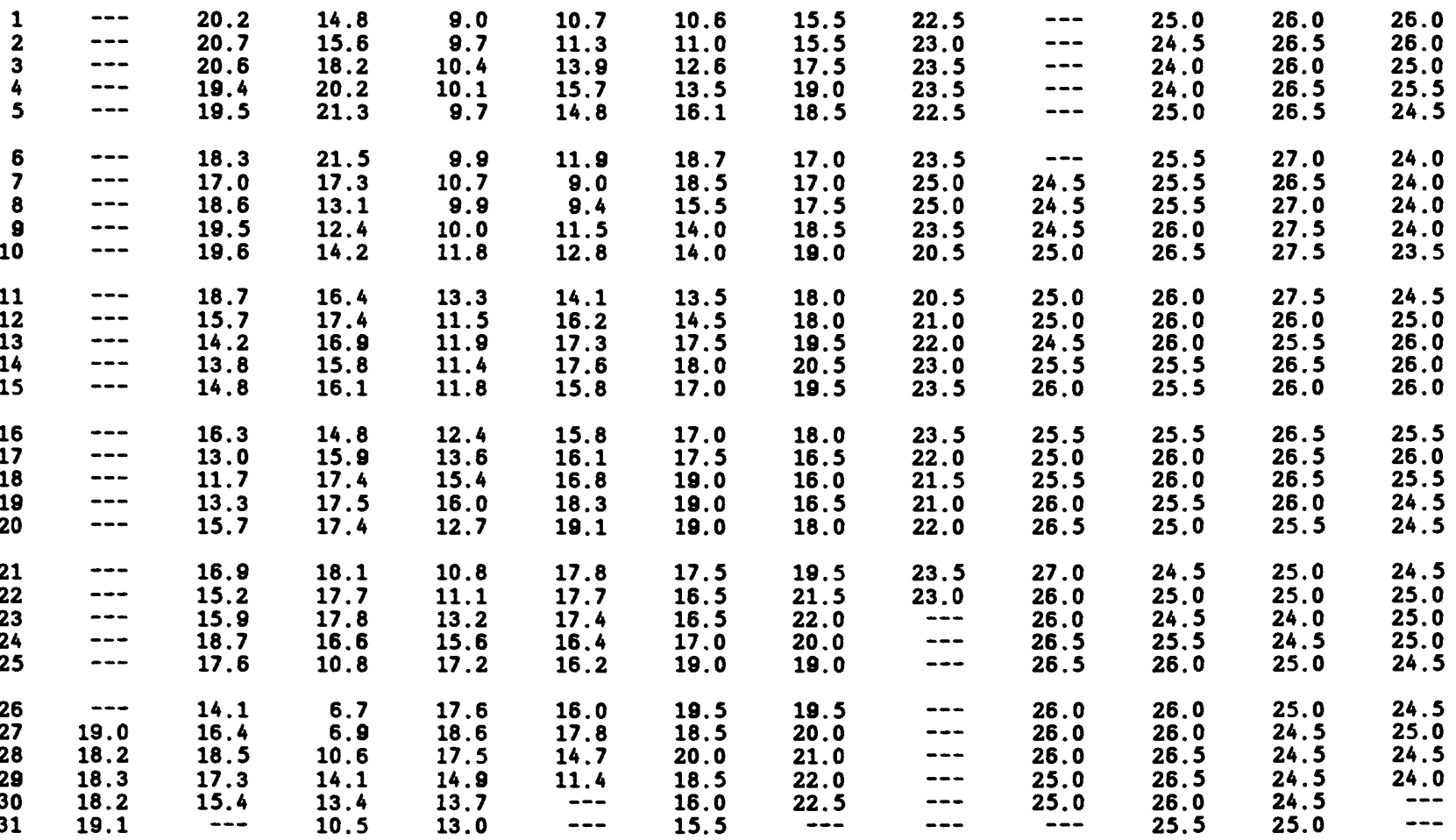


APPEIDIX V--Daily water temperature--Continued [Mean values in degrees Celsius]

\begin{tabular}{|c|c|c|c|c|c|c|c|c|c|c|c|c|}
\hline Day & Oct & Hov & Dec & $\operatorname{Jan}$ & Feb & Mar & Apr & May & June & July & Aus & Sept \\
\hline & \multicolumn{12}{|c|}{$\begin{array}{l}\text { 1--Tomoka River near Bolly Bill } \\
\text { year October } 1984 \text { to September 1985] }\end{array}$} \\
\hline $\begin{array}{l}1 \\
2 \\
3 \\
4 \\
5\end{array}$ & $\begin{array}{l}--- \\
--- \\
--- \\
--- \\
---\end{array}$ & $\begin{array}{l}--- \\
--- \\
--- \\
---\end{array}$ & $\begin{array}{l}--- \\
--- \\
--- \\
---\end{array}$ & $\begin{array}{l}--- \\
--- \\
--- \\
--- \\
---\end{array}$ & $\begin{array}{l}--- \\
-\cdots \\
-\cdots \\
---\end{array}$ & $\begin{array}{l}19.4 \\
19.8 \\
18.8 \\
19.1 \\
21.2\end{array}$ & $\begin{array}{l}20.9 \\
18.5 \\
17.7 \\
18.3 \\
19.7\end{array}$ & $\begin{array}{l}24.0 \\
23.7 \\
23.9 \\
23.1 \\
21.5\end{array}$ & $\begin{array}{l}--- \\
--- \\
--- \\
--- \\
---\end{array}$ & $\begin{array}{l}25.1 \\
25.9 \\
26.2 \\
26.0 \\
26.6\end{array}$ & $\begin{array}{l}25.7 \\
24.8 \\
25.1 \\
26.4 \\
26.7\end{array}$ & $\begin{array}{l}25.0 \\
25.0 \\
25.5 \\
25.6 \\
25.3\end{array}$ \\
\hline $\begin{array}{r}6 \\
7 \\
8 \\
9 \\
10\end{array}$ & $\begin{array}{l}--- \\
--- \\
--- \\
--- \\
---\end{array}$ & $\begin{array}{l}--- \\
--- \\
--- \\
---\end{array}$ & $\begin{array}{l}--- \\
--- \\
--- \\
---\end{array}$ & $\begin{array}{l}--- \\
--- \\
--- \\
--- \\
---\end{array}$ & $\begin{array}{l}--- \\
-- \\
--- \\
--- \\
---\end{array}$ & $\begin{array}{l}20.4 \\
20.3 \\
20.3 \\
20.2 \\
20.3\end{array}$ & $\begin{array}{l}20.3 \\
20.0 \\
20.3 \\
19.2 \\
17.5\end{array}$ & $\begin{array}{r}21.7 \\
21.9 \\
23.0 \\
--- \\
---\end{array}$ & $\begin{array}{l}--- \\
--- \\
--- \\
--- \\
---\end{array}$ & $\begin{array}{l}26.3 \\
26.6 \\
26.0 \\
26.8 \\
27.6\end{array}$ & $\begin{array}{l}26.5 \\
25.6 \\
24.8 \\
25.5 \\
25.5\end{array}$ & $\begin{array}{l}25.5 \\
25.5 \\
25.6 \\
25.4 \\
25.5\end{array}$ \\
\hline $\begin{array}{l}11 \\
12 \\
13 \\
14 \\
15\end{array}$ & $\begin{array}{l}--- \\
--- \\
--- \\
--- \\
---\end{array}$ & $\begin{array}{l}--- \\
--- \\
--- \\
---\end{array}$ & $\begin{array}{l}--- \\
--- \\
--- \\
---\end{array}$ & $\begin{array}{l}--- \\
--- \\
--- \\
--- \\
---\end{array}$ & $\begin{array}{l}-\ldots \\
-\ldots- \\
-\ldots \\
--\end{array}$ & $\begin{array}{l}19.8 \\
21.0 \\
21.4 \\
21.8 \\
21.3\end{array}$ & $\begin{array}{l}18.8 \\
19.5 \\
19.4 \\
19.9 \\
20.3\end{array}$ & $\begin{array}{l}--- \\
--- \\
--- \\
--- \\
---\end{array}$ & $\begin{array}{r}-. . \\
25.5 \\
24.3 \\
22.5\end{array}$ & $\begin{array}{l}27.9 \\
24.2 \\
24.6 \\
25.2 \\
26.1\end{array}$ & $\begin{array}{l}25.5 \\
25.4 \\
26.2 \\
25.5 \\
25.5\end{array}$ & $\begin{array}{l}25.6 \\
25.7 \\
25.4 \\
24.8 \\
24.0\end{array}$ \\
\hline $\begin{array}{l}16 \\
17 \\
18 \\
19 \\
20\end{array}$ & $\begin{array}{l}--- \\
--- \\
--- \\
--- \\
--\end{array}$ & $\begin{array}{l}--- \\
--- \\
--- \\
---\end{array}$ & $\begin{array}{l}--- \\
--- \\
--- \\
---\end{array}$ & $\begin{array}{l}--- \\
--- \\
--- \\
--- \\
---\end{array}$ & $\begin{array}{l}--- \\
--- \\
--- \\
---\end{array}$ & $\begin{array}{l}21.6 \\
20.4 \\
16.7 \\
15.4 \\
16.3\end{array}$ & $\begin{array}{l}20.5 \\
21.0 \\
21.4 \\
22.4 \\
22.4\end{array}$ & $\begin{array}{l}--- \\
--- \\
--- \\
--- \\
---\end{array}$ & $\begin{array}{l}24.1 \\
25.0 \\
26.2 \\
26.3 \\
26.5\end{array}$ & $\begin{array}{l}24.5 \\
24.8 \\
25.9 \\
25.7 \\
26.1\end{array}$ & $\begin{array}{l}25.4 \\
25.5 \\
25.9 \\
25.3 \\
25.5\end{array}$ & $\begin{array}{l}23.8 \\
22.6 \\
23.1 \\
23.4 \\
22.7\end{array}$ \\
\hline $\begin{array}{l}21 \\
22 \\
23 \\
24 \\
25\end{array}$ & $\begin{array}{l}--- \\
--- \\
--- \\
--- \\
---\end{array}$ & $\begin{array}{l}--- \\
--- \\
--- \\
---\end{array}$ & $\begin{array}{l}--- \\
--- \\
--- \\
---\end{array}$ & $\begin{array}{l}--- \\
--- \\
--- \\
--- \\
---\end{array}$ & $\begin{array}{l}--- \\
--- \\
--- \\
---\end{array}$ & $\begin{array}{l}17.4 \\
18.8 \\
19.0 \\
18.6 \\
19.1\end{array}$ & $\begin{array}{l}22.2 \\
23.3 \\
23.4 \\
24.2 \\
24.0\end{array}$ & $\begin{array}{l}=- \\
--- \\
--- \\
---\end{array}$ & $\begin{array}{l}25.8 \\
26.1 \\
26.2 \\
25.7 \\
25.8\end{array}$ & $\begin{array}{l}26.2 \\
25.8 \\
25.6 \\
25.1 \\
24.8\end{array}$ & $\begin{array}{l}25.5 \\
25.4 \\
25.3 \\
25.4 \\
25.4\end{array}$ & $\begin{array}{l}23.1 \\
24.8 \\
25.2 \\
25.3 \\
25.5\end{array}$ \\
\hline $\begin{array}{l}26 \\
27 \\
28 \\
29 \\
30 \\
31\end{array}$ & $\begin{array}{l}--- \\
-- \\
--- \\
-- \\
-- \\
--\end{array}$ & $\begin{array}{l}--- \\
--- \\
--- \\
--- \\
---\end{array}$ & $\begin{array}{l}--- \\
--- \\
--- \\
--- \\
---\end{array}$ & $\begin{array}{l}--- \\
--- \\
--- \\
-- \\
--- \\
--\end{array}$ & $\begin{array}{r}--- \\
19.9 \\
19.9 \\
--- \\
---\end{array}$ & $\begin{array}{l}18.6 \\
18.5 \\
19.4 \\
20.5 \\
21.3 \\
21.8\end{array}$ & $\begin{array}{r}24.0 \\
23.9 \\
24.0 \\
24.6 \\
24.8 \\
-\end{array}$ & $\begin{array}{l}--- \\
--- \\
--- \\
--- \\
--- \\
---\end{array}$ & $\begin{array}{r}25.9 \\
26.4 \\
25.3 \\
25.0 \\
25.4 \\
-\end{array}$ & $\begin{array}{l}25.5 \\
26.3 \\
26.4 \\
26.5 \\
26.3 \\
26.3\end{array}$ & $\begin{array}{l}26.0 \\
25.4 \\
25.7 \\
26.1 \\
26.0 \\
25.3\end{array}$ & $\begin{array}{r}25.5 \\
25.5 \\
25.2 \\
25.1 \\
25.4 \\
-\end{array}$ \\
\hline
\end{tabular}

Site 2--Eleventh Street Canal near Bolly Bill

[Water year October 1983 to September 1984]

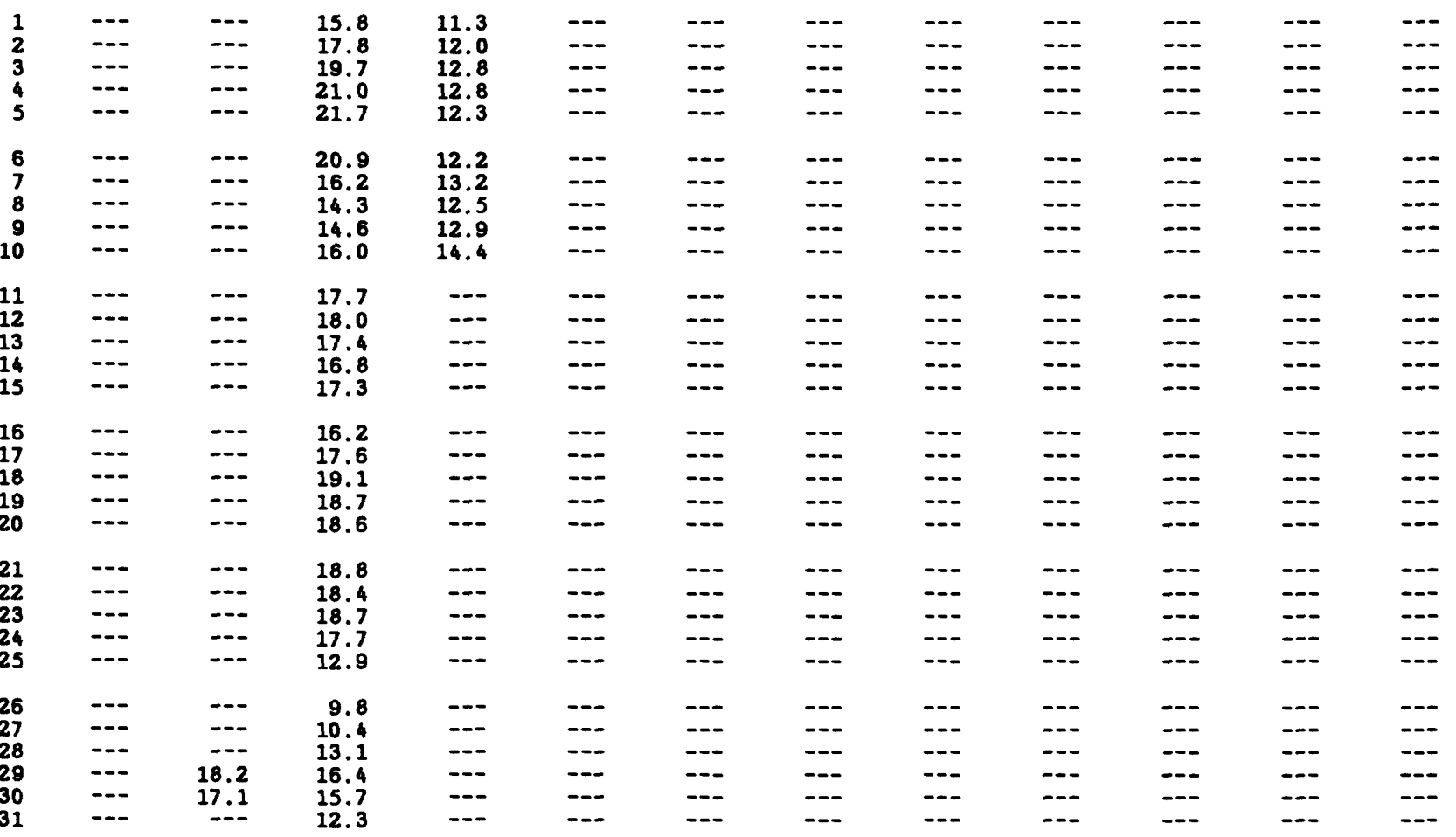


APPENDIX V--Daily water temperature--Continued

[Mean values in degrees Celsius]

\begin{tabular}{|c|c|c|c|c|c|c|c|c|c|c|c|c|}
\hline Day & Oct & Rov & Dec & $\operatorname{Jan}$ & Feb & Mar & Apr & May & June & July & Aus & Sept \\
\hline & \multicolumn{12}{|c|}{$\begin{array}{l}\text { Site 3--Williamson Boulevard Ditch at Daytona Beach } \\
\text { [Water year October } 1983 \text { to September 1984] }\end{array}$} \\
\hline $\begin{array}{l}1 \\
2 \\
3 \\
4 \\
5\end{array}$ & $\begin{array}{l}-- \\
-- \\
-- \\
-- \\
--\end{array}$ & $\begin{array}{l}--- \\
-- \\
-- \\
--\end{array}$ & $\begin{array}{l}--- \\
-- \\
--- \\
---\end{array}$ & $\begin{array}{l}--- \\
--- \\
--- \\
---\end{array}$ & $\begin{array}{l}--- \\
--- \\
--- \\
---\end{array}$ & $\begin{array}{l}--- \\
--- \\
--- \\
--- \\
--\end{array}$ & $\begin{array}{l}-- \\
-- \\
--- \\
-- \\
--\end{array}$ & $\begin{array}{l}-- \\
-- \\
-- \\
-- \\
--\end{array}$ & $\begin{array}{l}-- \\
-- \\
-- \\
-- \\
--\end{array}$ & $\begin{array}{l}-- \\
-- \\
-- \\
-- \\
--\end{array}$ & $\begin{array}{l}-- \\
--- \\
--\infty \\
--\infty \\
--\infty\end{array}$ & $\begin{array}{l}28.2 \\
28.0 \\
27.2 \\
27.1 \\
26.3\end{array}$ \\
\hline $\begin{array}{r}6 \\
7 \\
8 \\
9 \\
10\end{array}$ & $\begin{array}{l}--- \\
-- \\
--- \\
--- \\
---\end{array}$ & $\begin{array}{l}--- \\
--- \\
-- \\
---\end{array}$ & $\begin{array}{l}--- \\
--- \\
--- \\
---\end{array}$ & $\begin{array}{l}--- \\
--- \\
--- \\
---\end{array}$ & $\begin{array}{l}--- \\
-\cdots \\
--- \\
-\cdots\end{array}$ & $\begin{array}{l}--- \\
-\cdots \\
--- \\
---\end{array}$ & $\begin{array}{l}-- \\
-- \\
-- \\
--\end{array}$ & $\begin{array}{l}-- \\
-\infty \\
-\infty \\
--\infty \\
-\infty\end{array}$ & $\begin{array}{l}-- \\
-- \\
--- \\
--- \\
---\end{array}$ & $\begin{array}{l}-- \\
-- \\
-- \\
-- \\
--\end{array}$ & $\begin{array}{l}-- \\
--- \\
--- \\
--- \\
---\end{array}$ & $\begin{array}{l}25.5 \\
25.9 \\
25.6 \\
24.8 \\
24.6\end{array}$ \\
\hline $\begin{array}{l}11 \\
12 \\
13 \\
14 \\
15\end{array}$ & $\begin{array}{l}-- \\
-- \\
-- \\
-- \\
--\end{array}$ & $\begin{array}{l}--- \\
--- \\
--- \\
---\end{array}$ & $\begin{array}{l}--- \\
--- \\
-- \\
---\end{array}$ & $\begin{array}{l}--- \\
--- \\
--- \\
---\end{array}$ & $\begin{array}{l}--- \\
--- \\
--- \\
---\end{array}$ & $\begin{array}{l}--- \\
--- \\
--- \\
---\end{array}$ & $\begin{array}{l}-- \\
-- \\
-- \\
-- \\
--\end{array}$ & $\begin{array}{l}--- \\
--- \\
--- \\
--- \\
---\end{array}$ & $\begin{array}{l}--- \\
--- \\
--- \\
--- \\
---\end{array}$ & $\begin{array}{l}-- \\
-- \\
-- \\
-- \\
--\end{array}$ & $\begin{array}{l}-- \\
--- \\
--- \\
-- \\
--\end{array}$ & $\begin{array}{l}26.0 \\
26.9 \\
27.3 \\
27.6 \\
27.1\end{array}$ \\
\hline $\begin{array}{l}16 \\
17 \\
18 \\
19 \\
20\end{array}$ & $\begin{array}{l}-- \\
-- \\
-- \\
--- \\
---\end{array}$ & $\begin{array}{l}--- \\
--- \\
--- \\
---\end{array}$ & $\begin{array}{l}--- \\
--- \\
--- \\
---\end{array}$ & $\begin{array}{l}--- \\
-- \\
--- \\
---\end{array}$ & $\begin{array}{l}--- \\
--- \\
--- \\
---\end{array}$ & $\begin{array}{l}--- \\
--- \\
--- \\
---\end{array}$ & $\begin{array}{l}-- \\
-- \\
-- \\
-- \\
--\end{array}$ & $\begin{array}{l}-- \\
--- \\
--- \\
--- \\
---\end{array}$ & $\begin{array}{l}-- \\
--- \\
-- \\
--- \\
---\end{array}$ & $\begin{array}{l}-- \\
--- \\
--- \\
--- \\
---\end{array}$ & $\begin{array}{l}28.3 \\
28.3 \\
26.5 \\
25.4 \\
26.3\end{array}$ & $\begin{array}{l}27.1 \\
27.0 \\
26.0 \\
25.3 \\
25.7\end{array}$ \\
\hline $\begin{array}{l}21 \\
22 \\
23 \\
24 \\
25\end{array}$ & $\begin{array}{l}-- \\
--- \\
-- \\
--- \\
---\end{array}$ & $\begin{array}{l}--- \\
--- \\
--- \\
---\end{array}$ & $\begin{array}{l}--- \\
--- \\
--- \\
---\end{array}$ & $\begin{array}{l}--- \\
--- \\
--- \\
---\end{array}$ & $\begin{array}{l}--- \\
--- \\
--- \\
---\end{array}$ & $\begin{array}{l}--- \\
--- \\
--- \\
---\end{array}$ & $\begin{array}{l}-- \\
-- \\
--- \\
-- \\
--\end{array}$ & $\begin{array}{l}-- \\
--- \\
-- \\
--- \\
---\end{array}$ & $\begin{array}{l}-- \\
--- \\
-- \\
--- \\
---\end{array}$ & $\begin{array}{l}-- \\
--- \\
--- \\
--- \\
---\end{array}$ & $\begin{array}{l}26.2 \\
26.8 \\
26.6 \\
27.1 \\
27.1\end{array}$ & $\begin{array}{l}26.0 \\
26.2 \\
26.0 \\
25.6 \\
24.6\end{array}$ \\
\hline $\begin{array}{l}26 \\
27 \\
28 \\
29 \\
30 \\
31\end{array}$ & $\begin{array}{l}-- \\
-- \\
--- \\
--- \\
-- \\
--\end{array}$ & $\begin{array}{l}--- \\
--- \\
--- \\
--- \\
--- \\
--\end{array}$ & $\begin{array}{l}-- \\
-- \\
-- \\
-- \\
-- \\
--\end{array}$ & $\begin{array}{l}-- \\
--- \\
--- \\
-- \\
--- \\
--\end{array}$ & $\begin{array}{l}--- \\
--- \\
--- \\
--- \\
--- \\
--\end{array}$ & $\begin{array}{l}-- \\
-- \\
--- \\
--- \\
--- \\
--\end{array}$ & $\begin{array}{l}-- \\
-- \\
-- \\
-- \\
-- \\
--\end{array}$ & $\begin{array}{l}--- \\
--- \\
--- \\
-- \\
-- \\
--\end{array}$ & $\begin{array}{l}--- \\
-- \\
--- \\
-- \\
-- \\
--\end{array}$ & $\begin{array}{l}--- \\
-- \\
--- \\
--- \\
---\end{array}$ & $\begin{array}{l}27.6 \\
27.5 \\
27.5 \\
27.5 \\
27.4 \\
27.8\end{array}$ & $\begin{array}{r}25.5 \\
24.9 \\
24.2 \\
24.9 \\
24.9 \\
-\end{array}$ \\
\hline
\end{tabular}

Site 6--Bayless Boulevard Canal at Daytona Beach [Water year October 1983 to September 1984 ]

\begin{tabular}{|c|c|c|c|c|c|c|c|c|c|c|c|c|}
\hline $\begin{array}{l}1 \\
2 \\
3 \\
4 \\
5\end{array}$ & $\begin{array}{l}--- \\
--- \\
--- \\
--- \\
---\end{array}$ & $\begin{array}{l}--- \\
--- \\
--- \\
---\end{array}$ & $\begin{array}{l}15.9 \\
14.5 \\
16.0 \\
20.2 \\
21.0\end{array}$ & $\begin{array}{l}10.8 \\
12.0 \\
12.5 \\
12.0 \\
11.7\end{array}$ & $\begin{array}{l}11.4 \\
11.8 \\
14.3 \\
14.6 \\
12.1\end{array}$ & $\begin{array}{l}12.3 \\
11.9 \\
13.5 \\
15.0 \\
18.0\end{array}$ & $\begin{array}{l}--- \\
--- \\
--- \\
---\end{array}$ & $\begin{array}{l}--- \\
-- \\
-- \\
-- \\
---\end{array}$ & $\begin{array}{l}--- \\
--- \\
--- \\
--- \\
---\end{array}$ & $\begin{array}{l}--- \\
--- \\
-\cdots \\
---\end{array}$ & $\begin{array}{l}--\overline{-} \\
--\overline{-} \\
--- \\
--\overline{-}\end{array}$ & $\begin{array}{l}-- \\
-- \\
-- \\
-- \\
--\end{array}$ \\
\hline $\begin{array}{r}6 \\
7 \\
8 \\
9 \\
10\end{array}$ & $\begin{array}{l}--- \\
--- \\
--- \\
--- \\
---\end{array}$ & $\begin{array}{l}--- \\
--- \\
--- \\
--- \\
---\end{array}$ & $\begin{array}{l}21.3 \\
16.3 \\
13.4 \\
12.9 \\
14.3\end{array}$ & $\begin{array}{l}11.9 \\
12.6 \\
11.6 \\
12.3 \\
15.6\end{array}$ & $\begin{array}{r}11.0 \\
11.8 \\
12.8\end{array}$ & $\begin{array}{r}19.8 \\
19.6 \\
15.4 \\
13.3 \\
-\end{array}$ & $\begin{array}{l}--- \\
--- \\
--- \\
--- \\
---\end{array}$ & $\begin{array}{l}--- \\
--- \\
--- \\
---\end{array}$ & $\begin{array}{l}--- \\
--- \\
--- \\
---\end{array}$ & $\begin{array}{l}--- \\
--- \\
--- \\
---\end{array}$ & $\begin{array}{l}--- \\
-- \\
-- \\
--\end{array}$ & $\begin{array}{l}--- \\
--- \\
--- \\
--- \\
---\end{array}$ \\
\hline $\begin{array}{l}11 \\
12 \\
13 \\
14 \\
15\end{array}$ & $\begin{array}{l}--- \\
-- \\
-- \\
-- \\
---\end{array}$ & $\begin{array}{l}--- \\
--- \\
--- \\
---\end{array}$ & $\begin{array}{l}14.7 \\
15.6 \\
13.3 \\
14.5 \\
13.3\end{array}$ & $\begin{array}{r}15.2 \\
--0 \\
--2 \\
13.2 \\
13.8\end{array}$ & $\begin{array}{l}14.0 \\
16.2 \\
18.1 \\
18.7 \\
17.1\end{array}$ & $\begin{array}{l}--- \\
--- \\
-- \\
-- \\
---\end{array}$ & $\begin{array}{l}--- \\
--\overline{-} \\
--- \\
--\overline{-}\end{array}$ & $\begin{array}{l}--- \\
--- \\
--- \\
---\end{array}$ & $\begin{array}{l}--- \\
-\cdots \\
-- \\
--- \\
--\end{array}$ & $\begin{array}{l}--- \\
--- \\
--- \\
--\overline{-}\end{array}$ & $\begin{array}{l}-- \\
-- \\
-- \\
--\end{array}$ & $\begin{array}{l}--- \\
--- \\
--- \\
--\end{array}$ \\
\hline $\begin{array}{l}16 \\
17 \\
18 \\
19 \\
20\end{array}$ & $\begin{array}{l}--- \\
-- \\
--- \\
--- \\
---\end{array}$ & $\begin{array}{l}-- \\
-- \\
-- \\
--\end{array}$ & $\begin{array}{l}15.8 \\
14.5 \\
17.8 \\
15.4 \\
16.3\end{array}$ & $\begin{array}{l}14.3 \\
15.5 \\
14.6 \\
13.6 \\
13.9\end{array}$ & $\begin{array}{l}16.7 \\
16.5 \\
16.5 \\
17.6 \\
19.0\end{array}$ & $\begin{array}{l}--- \\
--- \\
--- \\
--- \\
---\end{array}$ & $\begin{array}{l}--- \\
--- \\
--- \\
---\end{array}$ & $\begin{array}{l}-- \\
-- \\
-- \\
--\end{array}$ & $\begin{array}{l}--- \\
--- \\
-- \\
--\end{array}$ & $\begin{array}{l}--- \\
--- \\
--- \\
---\end{array}$ & $\begin{array}{l}--- \\
-- \\
-- \\
--\end{array}$ & $\begin{array}{l}--- \\
--- \\
--- \\
---\end{array}$ \\
\hline $\begin{array}{l}21 \\
22 \\
23 \\
24 \\
25\end{array}$ & $\begin{array}{l}-- \\
--- \\
--- \\
--- \\
---\end{array}$ & $\begin{array}{r}16.4 \\
14.1 \\
16.9 \\
15.3\end{array}$ & $\begin{array}{l}16.3 \\
17.0 \\
18.7 \\
15.1 \\
10.3\end{array}$ & $\begin{array}{l}12.0 \\
12.5 \\
13.3 \\
14.4 \\
15.1\end{array}$ & $\begin{array}{l}18.6 \\
18.7 \\
18.6 \\
17.2 \\
16.9\end{array}$ & $\begin{array}{l}--- \\
--- \\
--- \\
--- \\
---\end{array}$ & $\begin{array}{l}--- \\
--- \\
--- \\
---\end{array}$ & $\begin{array}{l}-- \\
--- \\
--- \\
---\end{array}$ & $\begin{array}{l}--- \\
-- \\
-- \\
--\end{array}$ & $\begin{array}{l}--- \\
-- \\
-- \\
--\end{array}$ & $\begin{array}{l}--- \\
--- \\
--- \\
---\end{array}$ & $\begin{array}{l}--- \\
--- \\
--- \\
--- \\
---\end{array}$ \\
\hline $\begin{array}{l}26 \\
27 \\
28 \\
29 \\
30 \\
31\end{array}$ & $\begin{array}{l}--- \\
--- \\
--- \\
--- \\
--- \\
---\end{array}$ & $\begin{array}{r}14.4 \\
14.3 \\
15.9 \\
14.0 \\
16.3 \\
-\end{array}$ & $\begin{array}{r}7.8 \\
9.7 \\
14.5 \\
16.4 \\
13.5 \\
11.7\end{array}$ & $\begin{array}{l}15.2 \\
17.2 \\
13.5 \\
14.9 \\
14.1 \\
13.4\end{array}$ & $\begin{array}{r}16.2 \\
18.6 \\
17.0 \\
13.3 \\
- \\
-\end{array}$ & $\begin{array}{l}--- \\
--- \\
--- \\
--- \\
--- \\
--\end{array}$ & $\begin{array}{l}--- \\
--- \\
--- \\
--- \\
--\end{array}$ & $\begin{array}{l}-- \\
-- \\
-- \\
-- \\
-- \\
--\end{array}$ & $\begin{array}{l}--- \\
--- \\
--- \\
--- \\
-\cdots\end{array}$ & $\begin{array}{l}--- \\
--- \\
--- \\
--- \\
--- \\
---\end{array}$ & $\begin{array}{l}--- \\
--- \\
--- \\
--- \\
--- \\
--\end{array}$ & $\begin{array}{l}--- \\
-- \\
-- \\
-- \\
--\end{array}$ \\
\hline
\end{tabular}


APPENDIX V--Daily water temperature--Continued

[Mean values in degrees Celsius]

\begin{tabular}{|c|c|c|c|c|c|c|c|c|c|c|c|c|}
\hline Day & Oct & Nov & $\overline{D_{e c}}$ & Jan & $\overline{\text { Feb }}$ & Mar & $\overline{\text { Apr }}$ & May & June & July & Aus & Sept \\
\hline & \multicolumn{12}{|c|}{$\begin{array}{l}\text { 12--B-19 Canal at Dunlawton Avenue at Port Orange } \\
\text { [Water year October } 1982 \text { to September 1983] }\end{array}$} \\
\hline $\begin{array}{l}1 \\
2 \\
3 \\
4 \\
5\end{array}$ & $\begin{array}{l}-\infty \\
-- \\
-- \\
-\infty\end{array}$ & $m$ & $\begin{array}{l}-- \\
-- \\
-- \\
-- \\
--\end{array}$ & $\begin{array}{l}-- \\
--- \\
-- \\
--\end{array}$ & $\begin{array}{l}-- \\
-- \\
-- \\
-- \\
--\end{array}$ & $\begin{array}{r}17.2 \\
-17.9 \\
18.4 \\
19.4\end{array}$ & $\begin{array}{l}19.4 \\
19.2 \\
19.5 \\
19.6 \\
20.8\end{array}$ & $\begin{array}{l}22.1 \\
22.3 \\
23.1 \\
21.9 \\
20.7\end{array}$ & $\begin{array}{l}24.5 \\
25.4 \\
25.9 \\
26.1 \\
24.9\end{array}$ & $\begin{array}{l}26.9 \\
26.6 \\
26.2 \\
26.8 \\
26.8\end{array}$ & $\begin{array}{l}27.5 \\
28.5 \\
29.0 \\
28.0 \\
26.9\end{array}$ & $\begin{array}{l}26.0 \\
27.1 \\
27.4 \\
28.9 \\
29.6\end{array}$ \\
\hline $\begin{array}{r}6 \\
7 \\
8 \\
9 \\
10\end{array}$ & $\begin{array}{l}-\infty \\
-\infty \\
-\infty \\
-\infty\end{array}$ & $m$ & $\begin{array}{l}-\infty \\
-- \\
-\infty \\
--\infty \\
-\infty\end{array}$ & $\begin{array}{l}-- \\
--- \\
--- \\
--\end{array}$ & $\begin{array}{l}-- \\
-- \\
-- \\
--\end{array}$ & $\begin{array}{l}21.0 \\
20.3 \\
19.7 \\
19.9 \\
18.2\end{array}$ & $\begin{array}{l}21.8 \\
22.3 \\
23.0 \\
22.8 \\
22.0\end{array}$ & $\begin{array}{l}21.0 \\
21.4 \\
21.6 \\
22.0 \\
22.5\end{array}$ & $\begin{array}{l}24.8 \\
25.1 \\
25.1 \\
26.4 \\
26.1\end{array}$ & $\begin{array}{l}27.0 \\
27.1 \\
26.8 \\
27.0 \\
26.6\end{array}$ & $\begin{array}{l}27.8 \\
27.4 \\
27.1 \\
27.2 \\
27.5\end{array}$ & $\begin{array}{r}29.5 \\
28.4 \\
28.1 \\
28.4\end{array}$ \\
\hline $\begin{array}{l}11 \\
12 \\
13 \\
14 \\
15\end{array}$ & $\begin{array}{l}-\infty \\
-- \\
-\infty \\
-\infty\end{array}$ & $=-$ & $\begin{array}{l}-\infty \\
-- \\
-- \\
-- \\
--\end{array}$ & $-\infty$ & $\begin{array}{l}16.7 \\
15.8 \\
15.3 \\
14.0 \\
14.2\end{array}$ & $\begin{array}{l}15.2 \\
14.8 \\
15.0 \\
15.6 \\
17.0\end{array}$ & $\begin{array}{l}21.6 \\
21.4 \\
21.4 \\
21.7 \\
21.2\end{array}$ & $\begin{array}{l}22.6 \\
22.8 \\
22.7 \\
22.5 \\
22.9\end{array}$ & $\begin{array}{l}26.3 \\
26.6 \\
25.6 \\
25.4 \\
25.6\end{array}$ & $\begin{array}{l}26.2 \\
26.4 \\
26.6 \\
26.6 \\
27.1\end{array}$ & $\begin{array}{l}28.5 \\
28.4 \\
27.1 \\
26.4 \\
26.3\end{array}$ & $\begin{array}{l}28.0 \\
28.0 \\
27.3 \\
26.1 \\
26.0\end{array}$ \\
\hline $\begin{array}{l}16 \\
17 \\
18 \\
19 \\
20\end{array}$ & $-\infty$ & $=-$ & $\begin{array}{l}-- \\
-- \\
--- \\
-- \\
--\end{array}$ & $=$ & $\begin{array}{l}15.1 \\
15.5 \\
15.3 \\
15.7 \\
16.9\end{array}$ & $\begin{array}{l}18.0 \\
19.1 \\
19.0 \\
19.0 \\
19.5\end{array}$ & $\begin{array}{l}20.8 \\
20.1 \\
19.4 \\
19.0 \\
19.3\end{array}$ & $\begin{array}{l}23.5 \\
24.3 \\
24.7 \\
24.1 \\
24.0\end{array}$ & $\begin{array}{l}25.5 \\
25.7 \\
25.6 \\
25.5 \\
25.6\end{array}$ & $\begin{array}{r}27.6 \\
27.9 \\
27.9 \\
27.7 \\
-\end{array}$ & $\begin{array}{l}26.4 \\
26.0 \\
25.7 \\
26.0 \\
26.1\end{array}$ & $\begin{array}{l}26.3 \\
25.0 \\
25.5 \\
26.8 \\
26.7\end{array}$ \\
\hline $\begin{array}{l}21 \\
22 \\
23 \\
24 \\
25\end{array}$ & $\begin{array}{l}-- \\
-- \\
-- \\
--\end{array}$ & -- & $\begin{array}{l}-- \\
-- \\
-- \\
--\end{array}$ & $\begin{array}{l}-- \\
-- \\
-- \\
-- \\
--\end{array}$ & $\begin{array}{l}17.6 \\
18.4 \\
18.8 \\
18.1 \\
17.9\end{array}$ & $\begin{array}{l}20.1 \\
18.4 \\
17.8 \\
18.4 \\
17.3\end{array}$ & $\begin{array}{l}19.4 \\
20.1 \\
20.7 \\
20.8 \\
20.5\end{array}$ & $\begin{array}{l}24.6 \\
24.8 \\
25.0 \\
25.3 \\
24.9\end{array}$ & $\begin{array}{l}25.6 \\
25.5 \\
26.1 \\
26.0 \\
26.3\end{array}$ & $\begin{array}{l}27.5 \\
27.6 \\
27.3 \\
27.9 \\
28.3\end{array}$ & $\begin{array}{r}26.2 \\
26.1 \\
26.1 \\
27.2\end{array}$ & $\begin{array}{l}27.3 \\
26.1 \\
24.4 \\
24.2 \\
24.3\end{array}$ \\
\hline $\begin{array}{l}26 \\
27 \\
28 \\
29 \\
30 \\
31\end{array}$ & 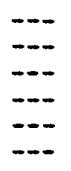 & $=-$ & $\begin{array}{l}-- \\
-- \\
-- \\
-- \\
--\end{array}$ & $\begin{array}{l}--- \\
-- \\
-- \\
-- \\
--\end{array}$ & $\begin{array}{r}15.7 \\
15.4 \\
17.2 \\
- \\
-\ldots\end{array}$ & $\begin{array}{l}17.3 \\
17.9 \\
19.2 \\
18.8 \\
18.6 \\
19.5\end{array}$ & $\begin{array}{l}20.4 \\
20.5 \\
20.9 \\
21.1 \\
21.3 \\
-\end{array}$ & $\begin{array}{l}24.7 \\
24.4 \\
24.6 \\
24.0 \\
23.0 \\
23.3\end{array}$ & $\begin{array}{r}27.0 \\
27.3 \\
27.0 \\
27.3 \\
27.3 \\
-.-\end{array}$ & $\begin{array}{l}27.8 \\
27.1 \\
27.6 \\
27.7 \\
27.2 \\
26.8\end{array}$ & $\begin{array}{l}26.4 \\
27.0 \\
27.0 \\
26.7 \\
26.9 \\
26.3\end{array}$ & $\begin{array}{r}24.3 \\
24.8 \\
23.9 \\
23.8 \\
24.0 \\
. .-\end{array}$ \\
\hline
\end{tabular}

Site 12--B-19 Canal at Dunlawton Avenue at Port Orange [Water year October 1983 to September 1984]

\begin{tabular}{|c|c|c|c|c|c|c|c|c|c|c|c|}
\hline $\begin{array}{l}24.4 \\
25.1 \\
25.0 \\
24.9 \\
24.0\end{array}$ & $\begin{array}{l}22.2 \\
22.5 \\
22.2 \\
21.3 \\
21.0\end{array}$ & $\begin{array}{l}17.5 \\
18.5 \\
20.9 \\
22.3 \\
22.6\end{array}$ & $\begin{array}{l}--- \\
--- \\
--- \\
---\end{array}$ & $=-$ & $\begin{array}{l}12.2 \\
13.2 \\
15.1 \\
16.3 \\
18.3\end{array}$ & -- & $\begin{array}{l}25.6 \\
26.1 \\
26.2 \\
25.9 \\
25.9\end{array}$ & $\begin{array}{l}-- \\
-- \\
-- \\
-- \\
--\end{array}$ & $\begin{array}{l}27.7 \\
27.4 \\
26.5 \\
26.5 \\
27.7\end{array}$ & $\begin{array}{l}--- \\
--- \\
--\end{array}$ & $\begin{array}{l}28.2 \\
28.0 \\
27.2 \\
27.1 \\
26.3\end{array}$ \\
\hline $\begin{array}{l}25.1 \\
25.3 \\
24.8 \\
25.6 \\
26.0\end{array}$ & $\begin{array}{l}20.1 \\
19.3 \\
21.0 \\
21.5 \\
21.6\end{array}$ & $\begin{array}{l}22.6 \\
18.1 \\
15.1 \\
15.5 \\
17.4\end{array}$ & $\begin{array}{l}--- \\
-- \\
-- \\
--\end{array}$ & $\begin{array}{r}-.- \\
13.1 \\
15.2 \\
16.4\end{array}$ & $\begin{array}{l}20.1 \\
19.3 \\
16.0 \\
15.2 \\
15.8\end{array}$ & -- & $\begin{array}{l}27.0 \\
28.0 \\
27.5 \\
24.9 \\
23.1\end{array}$ & $\begin{array}{r}-.- \\
26.8 \\
27.0\end{array}$ & $\begin{array}{l}28.3 \\
27.9 \\
28.2 \\
29.1 \\
29.2\end{array}$ & $=$ & $\begin{array}{l}25.5 \\
25.9 \\
25.6 \\
24.8 \\
24.6\end{array}$ \\
\hline $\begin{array}{l}25.1 \\
25.7 \\
26.0 \\
25.4 \\
25.1\end{array}$ & $\begin{array}{l}20.5 \\
17.8 \\
17.0 \\
17.0 \\
18.3\end{array}$ & $\begin{array}{l}19.2 \\
18.5 \\
18.0 \\
17.5 \\
17.8\end{array}$ & $\begin{array}{l}-- \\
-- \\
-- \\
--\end{array}$ & $\begin{array}{l}17.5 \\
19.1 \\
19.5 \\
19.3 \\
18.2\end{array}$ & $\begin{array}{r}15.6 \\
16.4 \\
\ldots \\
\ldots-\end{array}$ & $\begin{array}{r}-. . \\
23.1 \\
23.6 \\
22.0\end{array}$ & $\begin{array}{l}23.7 \\
24.9 \\
25.4 \\
25.9 \\
26.3\end{array}$ & $\begin{array}{l}26.4 \\
26.5 \\
25.9 \\
26.1 \\
27.1\end{array}$ & $\begin{array}{l}28.5 \\
28.4 \\
27.8 \\
27.7 \\
27.7\end{array}$ & $\begin{array}{l}-- \\
-- \\
-- \\
--\end{array}$ & $\begin{array}{l}26.0 \\
26.9 \\
27.3 \\
27.4 \\
27.1\end{array}$ \\
\hline $\begin{array}{l}24.9 \\
24.8 \\
24.6 \\
24.9 \\
25.3\end{array}$ & $\begin{array}{l}19.1 \\
15.8 \\
15.5 \\
17.4 \\
18.9\end{array}$ & $\begin{array}{l}16.7 \\
18.2 \\
19.3 \\
18.7 \\
18.5\end{array}$ & $\begin{array}{l}-\infty \\
--\infty \\
--\infty \\
--\infty\end{array}$ & $\begin{array}{l}18.0 \\
18.2 \\
18.7 \\
19.9 \\
20.8\end{array}$ & $\begin{array}{l}-m \\
--- \\
-\cdots \\
-\cdots\end{array}$ & $\begin{array}{l}21.2 \\
20.3 \\
20.4 \\
21.3 \\
22.8\end{array}$ & $\begin{array}{l}25.7 \\
24.2 \\
23.8 \\
23.8 \\
24.6\end{array}$ & $\begin{array}{l}26.8 \\
26.2 \\
26.6 \\
27.7 \\
28.2\end{array}$ & $\begin{array}{l}28.2 \\
28.5 \\
28.1 \\
26.9 \\
26.1\end{array}$ & $\begin{array}{l}28.3 \\
28.3 \\
26.5 \\
25.4 \\
26.3\end{array}$ & $\begin{array}{l}27.1 \\
27.0 \\
26.0 \\
25.3 \\
25.7\end{array}$ \\
\hline $\begin{array}{l}25.0 \\
24.6 \\
24.7 \\
24.8 \\
23.0\end{array}$ & $\begin{array}{l}19.4 \\
18.0 \\
19.0 \\
21.4 \\
19.3\end{array}$ & $\begin{array}{l}18.7 \\
18.5 \\
19.1 \\
17.8 \\
12.5\end{array}$ & $\begin{array}{l}-\infty \\
--\infty \\
-- \\
-- \\
-\infty\end{array}$ & $\begin{array}{l}19.9 \\
18.7 \\
18.6 \\
17.4 \\
17.5\end{array}$ & $\begin{array}{l}-- \\
--- \\
--\infty \\
--\infty\end{array}$ & $\begin{array}{l}23.8 \\
25.3 \\
24.3 \\
23.3 \\
22.9\end{array}$ & $\begin{array}{l}26.0 \\
26.3 \\
24.0 \\
24.4 \\
26.2\end{array}$ & $\begin{array}{l}28.6 \\
27.3 \\
26.4 \\
27.4 \\
27.4\end{array}$ & $\begin{array}{l}25.8 \\
26.7 \\
26.8 \\
28.0 \\
28.3\end{array}$ & $\begin{array}{l}26.2 \\
26.8 \\
26.6 \\
27.1 \\
27.1\end{array}$ & $\begin{array}{l}26.0 \\
26.2 \\
26.0 \\
25.6 \\
24.6\end{array}$ \\
\hline $\begin{array}{r}--- \\
21.2 \\
21.0 \\
21.1 \\
21.3 \\
21.9\end{array}$ & $\begin{array}{r}16.7 \\
19.5 \\
21.0 \\
19.0 \\
17.8 \\
-\end{array}$ & $\begin{array}{r}10.4 \\
12.2 \\
16.2 \\
16.7 \\
- \\
--\end{array}$ & $\begin{array}{l}-- \\
-- \\
-- \\
-- \\
--\end{array}$ & $\begin{array}{r}17.0 \\
18.6 \\
16.3 \\
12.7 \\
-.-0 \\
-.-\end{array}$ & $\begin{array}{l}-- \\
-- \\
-- \\
--\end{array}$ & $\begin{array}{l}23.5 \\
24.1 \\
25.1 \\
25.8 \\
25.6 \\
-\end{array}$ & $\begin{array}{l}25.4 \\
26.5 \\
27.1 \\
26.8 \\
25.5 \\
\end{array}$ & $\begin{array}{r}27.7 \\
28.3 \\
27.4 \\
26.3 \\
26.3 \\
-\end{array}$ & $\begin{array}{r}28.1 \\
27.7 \\
27.9 \\
27.8 \\
27.4 \\
-\ldots\end{array}$ & $\begin{array}{l}27.6 \\
27.5 \\
27.5 \\
27.5 \\
27.4 \\
27.8\end{array}$ & $\begin{array}{r}25.0 \\
24.8 \\
24.2 \\
24.9 \\
24.9 \\
-.0\end{array}$ \\
\hline
\end{tabular}


APPENDIX V--Daily water temperature--Continued [Mean values in degrees Celsius]

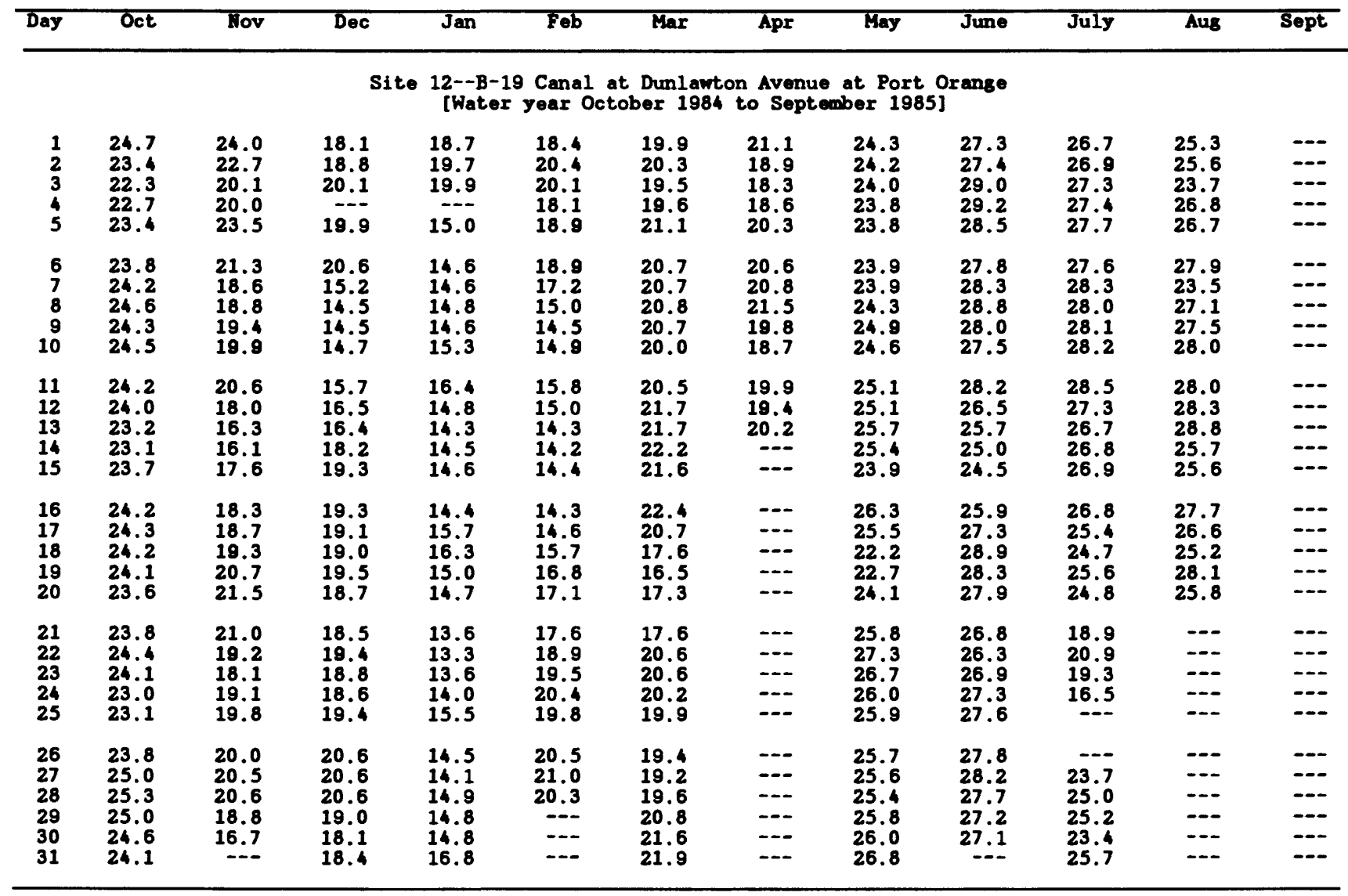


Appendix VI 
APPENDIX VI--Daily specific conductance

IMean values in microsiemens per centimeter at 25 degrees Celsius

\begin{tabular}{|c|c|c|c|c|c|c|c|c|c|c|c|c|}
\hline$\overline{\text { Day }}$ & Oct & Kov & Dec & Jan & Feb & Mar & Apr & May & June & July & Aug & Sept \\
\hline & \multicolumn{12}{|c|}{$\begin{array}{l}\text { Site 1--Tomoka River near Holly Bill } \\
\text { [Water year October } 1982 \text { to September 1983] }\end{array}$} \\
\hline $\begin{array}{l}1 \\
2 \\
3 \\
4 \\
5\end{array}$ & $\begin{array}{l}--- \\
-\cdots \\
--- \\
-- \\
--\end{array}$ & $\begin{array}{l}-- \\
--- \\
--- \\
-- \\
--\end{array}$ & $\begin{array}{l}-- \\
--- \\
--- \\
-- \\
--\end{array}$ & 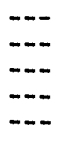 & 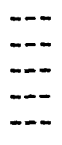 & $\begin{array}{l}84 \\
86 \\
86 \\
86 \\
87\end{array}$ & $\begin{array}{l}91 \\
84 \\
84 \\
82 \\
81\end{array}$ & $\frac{--}{--}$ & $\begin{array}{l}176 \\
210 \\
231 \\
253 \\
225\end{array}$ & $\begin{array}{l}83 \\
79 \\
78 \\
83 \\
86\end{array}$ & $\begin{array}{l}-- \\
--- \\
--- \\
-- \\
--\end{array}$ & $\begin{array}{l}183 \\
171 \\
162 \\
151 \\
133\end{array}$ \\
\hline $\begin{array}{r}6 \\
7 \\
8 \\
9 \\
10\end{array}$ & $\begin{array}{l}-- \\
-- \\
-- \\
-- \\
--\end{array}$ & $\begin{array}{l}--- \\
--- \\
-- \\
--\end{array}$ & $\begin{array}{l}--- \\
--- \\
-- \\
-- \\
--\end{array}$ & $m$ & $m$ & $\begin{array}{r}89 \\
93 \\
117 \\
128 \\
107\end{array}$ & $\begin{array}{r}80 \\
79 \\
101 \\
84 \\
93\end{array}$ & $\begin{array}{l}71 \\
71 \\
71 \\
73 \\
78\end{array}$ & $\begin{array}{r}214 \\
176 \\
123 \\
96 \\
107\end{array}$ & $\begin{array}{r}96 \\
102 \\
102 \\
124 \\
144\end{array}$ & $\begin{array}{l}--- \\
--- \\
293 \\
192 \\
136\end{array}$ & $\begin{array}{l}136 \\
136 \\
-- \\
---\end{array}$ \\
\hline $\begin{array}{l}11 \\
12 \\
13 \\
14 \\
15\end{array}$ & $\begin{array}{l}-- \\
-- \\
-- \\
--\end{array}$ & $\begin{array}{l}-- \\
-- \\
-- \\
--- \\
---\end{array}$ & $\begin{array}{l}-- \\
-- \\
--- \\
-- \\
--\end{array}$ & 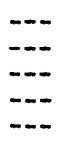 & $\begin{array}{r}107 \\
99 \\
110 \\
105 \\
93\end{array}$ & $\begin{array}{l}97 \\
93 \\
89 \\
87 \\
85\end{array}$ & $\begin{array}{l}85 \\
79 \\
75 \\
72 \\
72\end{array}$ & $\begin{array}{l}75 \\
79 \\
82 \\
91 \\
89\end{array}$ & $\begin{array}{l}116 \\
136 \\
136 \\
135 \\
131\end{array}$ & $\begin{array}{l}156 \\
196 \\
200 \\
152 \\
-\end{array}$ & $\begin{array}{l}177 \\
164 \\
148 \\
122 \\
121\end{array}$ & $\begin{array}{l}-- \\
-- \\
-- \\
--\end{array}$ \\
\hline $\begin{array}{l}16 \\
17 \\
18 \\
19 \\
20\end{array}$ & 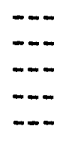 & $\begin{array}{l}-- \\
-- \\
-- \\
-- \\
--\end{array}$ & $\begin{array}{l}-- \\
-- \\
-- \\
-- \\
--\end{array}$ & $m$ & $\begin{array}{l}84 \\
93 \\
93 \\
83 \\
79\end{array}$ & $\begin{array}{l}97 \\
85 \\
78 \\
80 \\
81\end{array}$ & $\begin{array}{l}-- \\
-- \\
-- \\
-- \\
--\end{array}$ & $\begin{array}{r}87 \\
103 \\
114 \\
128 \\
142\end{array}$ & $\begin{array}{l}135 \\
137 \\
142 \\
147 \\
146\end{array}$ & $\begin{array}{l}-- \\
--- \\
-- \\
-- \\
--\end{array}$ & $\begin{array}{l}106 \\
106 \\
113 \\
122 \\
119\end{array}$ & $\begin{array}{l}-- \\
-- \\
-- \\
--\end{array}$ \\
\hline $\begin{array}{l}21 \\
22 \\
23 \\
24 \\
25\end{array}$ & $\begin{array}{l}-- \\
--- \\
-- \\
-- \\
---\end{array}$ & $\begin{array}{l}-- \\
--- \\
--- \\
--- \\
---\end{array}$ & $\begin{array}{l}-- \\
--- \\
--- \\
--- \\
---\end{array}$ & $\begin{array}{l}\cdots- \\
\cdots- \\
\cdots-\end{array}$ & $\begin{array}{l}77 \\
77 \\
78 \\
78 \\
79\end{array}$ & $\begin{array}{l}85 \\
84 \\
81 \\
83 \\
86\end{array}$ & $\begin{array}{r}-- \\
--- \\
---\end{array}$ & $\begin{array}{l}154 \\
170 \\
195 \\
202 \\
193\end{array}$ & $\begin{array}{l}137 \\
141 \\
114 \\
117 \\
105\end{array}$ & $\begin{array}{l}-- \\
-- \\
-- \\
-- \\
--\end{array}$ & $\begin{array}{l}117 \\
116 \\
112 \\
112 \\
113\end{array}$ & $\begin{array}{l}-- \\
-- \\
-- \\
--\end{array}$ \\
\hline $\begin{array}{l}26 \\
27 \\
28 \\
29 \\
30 \\
31\end{array}$ & $\begin{array}{l}--- \\
-- \\
-- \\
-- \\
--\end{array}$ & $\begin{array}{l}--- \\
-- \\
--- \\
-- \\
-- \\
--\end{array}$ & $\begin{array}{l}-- \\
--- \\
-- \\
-- \\
-- \\
--\end{array}$ & $\begin{array}{l}--- \\
--- \\
--- \\
--- \\
---\end{array}$ & $\begin{array}{r}80 \\
79 \\
80 \\
-- \\
-- \\
--\end{array}$ & $\begin{array}{l}81 \\
81 \\
91 \\
85 \\
82 \\
87\end{array}$ & $\begin{array}{l}-- \\
-- \\
--- \\
-- \\
--\end{array}$ & $\begin{array}{r}186 \\
117 \\
198 \\
93 \\
188 \\
160\end{array}$ & $\begin{array}{r}98 \\
96 \\
95 \\
94 \\
91 \\
---\end{array}$ & $\begin{array}{l}--- \\
--- \\
--- \\
-- \\
--\end{array}$ & $\begin{array}{l}130 \\
142 \\
140 \\
148 \\
163 \\
176\end{array}$ & $\begin{array}{l}-- \\
-- \\
-- \\
--\end{array}$ \\
\hline
\end{tabular}

Site 1--Tomoka River near Holly Hill

[Water year October 1983 to September 1984]

\begin{tabular}{|c|c|c|c|c|c|c|c|c|c|c|c|c|}
\hline $\begin{array}{l}1 \\
2 \\
3 \\
4 \\
5\end{array}$ & $\begin{array}{l}182 \\
182 \\
185 \\
187 \\
187\end{array}$ & $\begin{array}{l}94 \\
96 \\
96 \\
96 \\
97\end{array}$ & $\begin{array}{l}120 \\
113 \\
114 \\
117 \\
119\end{array}$ & $\begin{array}{l}70 \\
70 \\
71 \\
71 \\
71\end{array}$ & $\begin{array}{l}93 \\
94 \\
93 \\
97 \\
98\end{array}$ & $\begin{array}{l}81 \\
80 \\
79 \\
79 \\
79\end{array}$ & $\begin{array}{l}152 \\
161 \\
160 \\
112 \\
101\end{array}$ & $\begin{array}{l}133 \\
154 \\
158 \\
159 \\
161\end{array}$ & $\begin{array}{l}--- \\
--- \\
--- \\
--- \\
--\infty\end{array}$ & $\begin{array}{l}161 \\
102 \\
111 \\
116 \\
127\end{array}$ & $\begin{array}{r}104 \\
106 \\
94 \\
93 \\
86\end{array}$ & $\begin{array}{l}99 \\
97 \\
92 \\
91 \\
94\end{array}$ \\
\hline $\begin{array}{r}6 \\
7 \\
8 \\
9 \\
10\end{array}$ & $\begin{array}{l}194 \\
200 \\
192 \\
173 \\
185\end{array}$ & $\begin{array}{l}101 \\
101 \\
103 \\
102 \\
101\end{array}$ & $\begin{array}{l}121 \\
121 \\
120 \\
120 \\
122\end{array}$ & $\begin{array}{l}71 \\
71 \\
73 \\
73 \\
75\end{array}$ & $\begin{array}{l}101 \\
108 \\
114 \\
123 \\
124\end{array}$ & $\begin{array}{l}80 \\
80 \\
80 \\
81 \\
81\end{array}$ & $\begin{array}{r}107 \\
96 \\
92 \\
90 \\
93\end{array}$ & $\begin{array}{l}155 \\
155 \\
183 \\
207 \\
217\end{array}$ & $\begin{array}{l}-159 \\
159 \\
179 \\
199\end{array}$ & $\begin{array}{r}120 \\
110 \\
106 \\
101 \\
98\end{array}$ & $\begin{array}{l}85 \\
83 \\
87 \\
93 \\
89\end{array}$ & $\begin{array}{r}105 \\
86 \\
79 \\
81 \\
77\end{array}$ \\
\hline $\begin{array}{l}11 \\
12 \\
13 \\
14 \\
15\end{array}$ & $\begin{array}{l}183 \\
183 \\
161 \\
166 \\
166\end{array}$ & $\begin{array}{r}101 \\
100 \\
97 \\
94 \\
93\end{array}$ & $\begin{array}{r}124 \\
102 \\
78 \\
82 \\
91\end{array}$ & $\begin{array}{r}92 \\
101 \\
104 \\
105 \\
94\end{array}$ & $\begin{array}{l}127 \\
132 \\
128 \\
129 \\
120\end{array}$ & $\begin{array}{l}82 \\
82 \\
86 \\
94 \\
95\end{array}$ & $\begin{array}{l}91 \\
88 \\
86 \\
82 \\
97\end{array}$ & $\begin{array}{l}236 \\
258 \\
285 \\
295 \\
310\end{array}$ & $\begin{array}{l}199 \\
212 \\
228 \\
223 \\
147\end{array}$ & $\begin{array}{l}93 \\
86 \\
82 \\
81 \\
78\end{array}$ & $\begin{array}{r}89 \\
96 \\
122 \\
133 \\
125\end{array}$ & $\begin{array}{l}70 \\
72 \\
75 \\
80 \\
80\end{array}$ \\
\hline $\begin{array}{l}16 \\
17 \\
18 \\
19 \\
20\end{array}$ & $\begin{array}{r}132 \\
113 \\
93 \\
96 \\
100\end{array}$ & $\begin{array}{r}98 \\
102 \\
97 \\
94 \\
94\end{array}$ & $\begin{array}{r}105 \\
103 \\
107 \\
101 \\
90\end{array}$ & $\begin{array}{r}89 \\
87 \\
88 \\
94 \\
101\end{array}$ & $\begin{array}{r}108 \\
103 \\
99 \\
95 \\
93\end{array}$ & $\begin{array}{r}94 \\
98 \\
109 \\
117 \\
118\end{array}$ & $\begin{array}{l}92 \\
91 \\
88 \\
87 \\
89\end{array}$ & $\begin{array}{l}330 \\
337 \\
351 \\
365 \\
375\end{array}$ & $\begin{array}{l}--- \\
--- \\
238 \\
186 \\
135\end{array}$ & $\begin{array}{l}78 \\
77 \\
79 \\
84 \\
99\end{array}$ & $\begin{array}{l}126 \\
120 \\
128 \\
111 \\
115\end{array}$ & $\begin{array}{l}75 \\
72 \\
71 \\
80 \\
71\end{array}$ \\
\hline $\begin{array}{l}21 \\
22 \\
23 \\
24 \\
25\end{array}$ & $\begin{array}{l}102 \\
106 \\
108 \\
108 \\
105\end{array}$ & $\begin{array}{l}114 \\
110 \\
102 \\
103 \\
105\end{array}$ & $\begin{array}{r}78 \\
75 \\
76 \\
77 \\
162\end{array}$ & $\begin{array}{r}101 \\
105 \\
101 \\
100 \\
99\end{array}$ & $\begin{array}{r}94 \\
102 \\
100 \\
106 \\
110\end{array}$ & $\begin{array}{l}128 \\
135 \\
130 \\
126 \\
132\end{array}$ & $\begin{array}{l}89 \\
87 \\
86 \\
88 \\
97\end{array}$ & $\begin{array}{l}379 \\
385 \\
--- \\
--- \\
---\end{array}$ & $\begin{array}{l}--- \\
--- \\
181 \\
296 \\
223\end{array}$ & $\begin{array}{r}96 \\
101 \\
98 \\
103 \\
92\end{array}$ & $\begin{array}{r}124 \\
124 \\
100 \\
89 \\
97\end{array}$ & $\begin{array}{l}72 \\
72 \\
72 \\
72 \\
72\end{array}$ \\
\hline $\begin{array}{l}26 \\
27 \\
28 \\
29 \\
30 \\
31\end{array}$ & $\begin{array}{r}102 \\
103 \\
100 \\
96 \\
94 \\
94\end{array}$ & $\begin{array}{l}128 \\
126 \\
119 \\
113 \\
129 \\
---\end{array}$ & $\begin{array}{r}163 \\
161 \\
143 \\
129 \\
78 \\
70\end{array}$ & $\begin{array}{l}98 \\
97 \\
96 \\
94 \\
92 \\
93\end{array}$ & $\begin{array}{r}111 \\
97 \\
100 \\
85 \\
---\end{array}$ & $\begin{array}{l}133 \\
142 \\
156 \\
150 \\
150 \\
149\end{array}$ & $\begin{array}{r}99 \\
99 \\
103 \\
113 \\
121 \\
---\end{array}$ & $\begin{array}{l}--- \\
--- \\
--- \\
--- \\
--- \\
---\end{array}$ & $\begin{array}{l}246 \\
193 \\
188 \\
189 \\
189 \\
-\cdots\end{array}$ & $\begin{array}{r}91 \\
96 \\
107 \\
105 \\
94 \\
100\end{array}$ & $\begin{array}{r}115 \\
98 \\
87 \\
80 \\
79 \\
88\end{array}$ & $\begin{array}{r}72 \\
72 \\
71 \\
-- \\
--- \\
--\end{array}$ \\
\hline
\end{tabular}


APPENDIX VI--Daily specific conductance--Continued

[Mean values in microsiemens per centimeter at 25 degrees Celsius]

\begin{tabular}{|c|c|c|c|c|c|c|c|c|c|c|c|c|}
\hline Day & Oct & Iov & Dec & Jan & $F \Theta b$ & Mar & Apr & May & June & July & Aus & Sept \\
\hline \multicolumn{13}{|c|}{$\begin{array}{l}\text { Site 1--Tomoka River near Holly Bill } \\
\text { [Water year October } 1984 \text { to September 1985] }\end{array}$} \\
\hline $\begin{array}{l}1 \\
2 \\
3 \\
4 \\
5\end{array}$ & $\begin{array}{l}-\cdots \\
\cdots- \\
\cdots- \\
-\cdots\end{array}$ & $\begin{array}{l}-\cdots \\
\cdots-- \\
\cdots-- \\
\cdots-\end{array}$ & $\begin{array}{l}-\cdots \\
--- \\
-\cdots \\
-\cdots\end{array}$ & $\begin{array}{l}--- \\
-\cdots \\
-\cdots \\
-\cdots\end{array}$ & $\begin{array}{l}-- \\
-- \\
--- \\
---\end{array}$ & $\begin{array}{l}--- \\
--- \\
--- \\
-\cdots\end{array}$ & $\begin{array}{l}-- \\
-\cdots \\
-\cdots \\
-\cdots\end{array}$ & $\begin{array}{l}--- \\
-\cdots \\
340 \\
229\end{array}$ & $\begin{array}{l}-\cdots \\
-\cdots \\
\cdots-- \\
\cdots-\end{array}$ & $\begin{array}{r}223 \\
233 \\
237 \\
130 \\
80\end{array}$ & $\begin{array}{l}202 \\
162 \\
183 \\
175 \\
178\end{array}$ & $\begin{array}{l}--. \\
--- \\
-- \\
--\end{array}$ \\
\hline $\begin{array}{r}6 \\
7 \\
8 \\
9 \\
10\end{array}$ & \begin{tabular}{l}
$-\cdots$ \\
$-\cdots$ \\
\hdashline-- \\
$-\cdots$
\end{tabular} & $\begin{array}{l}-\cdots \\
-\cdots \\
-\cdots \\
-\cdots\end{array}$ & $\begin{array}{l}-\cdots \\
\cdots-- \\
\cdots-- \\
-\cdots\end{array}$ & $\begin{array}{l}\cdots- \\
\cdots- \\
\cdots- \\
\cdots-\end{array}$ & $\begin{array}{l}-- \\
-- \\
--- \\
---\end{array}$ & \begin{tabular}{l}
$-\cdots$ \\
\hdashline-- \\
\hdashline-- \\
$-\cdots$
\end{tabular} & \begin{tabular}{l}
$-\cdots$ \\
$\overline{-}$ \\
\hdashline-- \\
$-\cdots$
\end{tabular} & \begin{tabular}{l}
243 \\
170 \\
\hdashline-- \\
\hdashline--
\end{tabular} & \begin{tabular}{l}
$-\cdots$ \\
$\cdots--$ \\
\hdashline-- \\
$-\cdots$
\end{tabular} & $\begin{array}{l}244 \\
233 \\
213 \\
215 \\
178\end{array}$ & $\begin{array}{l}147 \\
147 \\
111 \\
110 \\
112\end{array}$ & $\begin{array}{l}--- \\
--- \\
\cdots-- \\
-\cdots\end{array}$ \\
\hline $\begin{array}{l}11 \\
12 \\
13 \\
14 \\
15\end{array}$ & $\begin{array}{l}-- \\
\cdots- \\
-\cdots\end{array}$ & $\begin{array}{l}\cdots \\
\cdots \\
\cdots-\end{array}$ & $\begin{array}{l}-- \\
-\cdots \\
\cdots-\end{array}$ & $\begin{array}{l}-- \\
-\cdots \\
\cdots-- \\
-\cdots\end{array}$ & $\begin{array}{l}-- \\
-- \\
\cdots-\end{array}$ & $\begin{array}{l}--- \\
\cdots-- \\
\cdots--\end{array}$ & $\begin{array}{l}-2 \\
167 \\
238 \\
250\end{array}$ & $\begin{array}{l}-- \\
-\cdots \\
-\cdots\end{array}$ & $\begin{array}{l}-- \\
-187 \\
198 \\
163\end{array}$ & $\begin{array}{l}123 \\
159 \\
245 \\
254 \\
235\end{array}$ & $\begin{array}{l}105 \\
104 \\
113 \\
109 \\
109\end{array}$ & $\bar{m}$ \\
\hline $\begin{array}{l}16 \\
17 \\
18 \\
19 \\
20\end{array}$ & $\begin{array}{l}\cdots- \\
\cdots- \\
\cdots- \\
\cdots-\end{array}$ & $\begin{array}{l}--- \\
--- \\
-\cdots \\
---\end{array}$ & $\begin{array}{l}\cdots \\
\cdots \\
\cdots \\
\cdots\end{array}$ & $\begin{array}{l}--- \\
-\cdots \\
--- \\
-\cdots\end{array}$ & $\begin{array}{l}-- \\
\cdots \\
\cdots- \\
\cdots-\end{array}$ & $\begin{array}{l}-- \\
\cdots \\
-\cdots \\
-\cdots\end{array}$ & $\begin{array}{l}145 \\
-- \\
-- \\
--\end{array}$ & $\begin{array}{l}--- \\
-\cdots \\
--- \\
---\end{array}$ & $\begin{array}{l}146 \\
152 \\
164 \\
201 \\
230\end{array}$ & $\begin{array}{l}167 \\
214 \\
187 \\
153 \\
169\end{array}$ & $\begin{array}{r}101 \\
95 \\
89 \\
83 \\
-\end{array}$ & $\begin{array}{r}--- \\
85 \\
76 \\
81\end{array}$ \\
\hline $\begin{array}{l}21 \\
22 \\
23 \\
24 \\
25\end{array}$ & $\begin{array}{l}--- \\
\cdots-- \\
-\cdots \\
-\cdots\end{array}$ & $\begin{array}{l}\cdots- \\
\cdots \\
\cdots-- \\
\cdots\end{array}$ & $\begin{array}{l}\cdots- \\
\cdots-- \\
\cdots-- \\
\cdots\end{array}$ & $\begin{array}{l}--- \\
--- \\
-\cdots \\
-\cdots\end{array}$ & $\begin{array}{l}\cdots- \\
\cdots- \\
\cdots- \\
\cdots-\end{array}$ & $\begin{array}{l}--- \\
327 \\
220 \\
---\end{array}$ & \begin{tabular}{l}
-- \\
-- \\
\hdashline- \\
$-\cdots$
\end{tabular} & $\begin{array}{l}--- \\
--- \\
--- \\
---\end{array}$ & $\begin{array}{l}250 \\
255 \\
268 \\
245 \\
212\end{array}$ & $\begin{array}{l}176 \\
178 \\
183 \\
163 \\
146\end{array}$ & $\begin{array}{l}\cdots- \\
\cdots-- \\
\cdots--\end{array}$ & $\begin{array}{l}\cdots- \\
\cdots \\
\cdots-\end{array}$ \\
\hline $\begin{array}{l}26 \\
27 \\
28 \\
29 \\
30 \\
31\end{array}$ & $\begin{array}{l}\cdots \\
-\cdots \\
\cdots- \\
\cdots- \\
-\cdots\end{array}$ & $\begin{array}{l}\cdots \\
--- \\
\cdots \\
\cdots \\
\cdots\end{array}$ & $\begin{array}{l}--- \\
\cdots- \\
\cdots- \\
\cdots- \\
\cdots\end{array}$ & $\begin{array}{l}--- \\
-\cdots \\
-\cdots \\
--- \\
-\cdots\end{array}$ & $\begin{array}{l}\cdots- \\
\cdots- \\
\cdots- \\
\cdots-\end{array}$ & $\begin{array}{l}--- \\
--- \\
--- \\
-\cdots \\
\cdots-\end{array}$ & $\begin{array}{l}\cdots \\
\cdots- \\
\cdots- \\
\cdots\end{array}$ & $\begin{array}{l}--- \\
--- \\
-\cdots \\
--- \\
-\cdots\end{array}$ & $\begin{array}{l}208 \\
231 \\
259 \\
271 \\
255 \\
-\cdots\end{array}$ & $\begin{array}{l}198 \\
204 \\
204 \\
207 \\
210 \\
215\end{array}$ & $\begin{array}{l}-\cdots \\
-\cdots \\
\cdots- \\
\cdots- \\
-\cdots\end{array}$ & $\begin{array}{l}-\cdot- \\
-- \\
-- \\
-- \\
-- \\
--\end{array}$ \\
\hline
\end{tabular}

Site 2--Eleventh Street Canal near Bolly Bill [Water year October 1983 to September 1984]

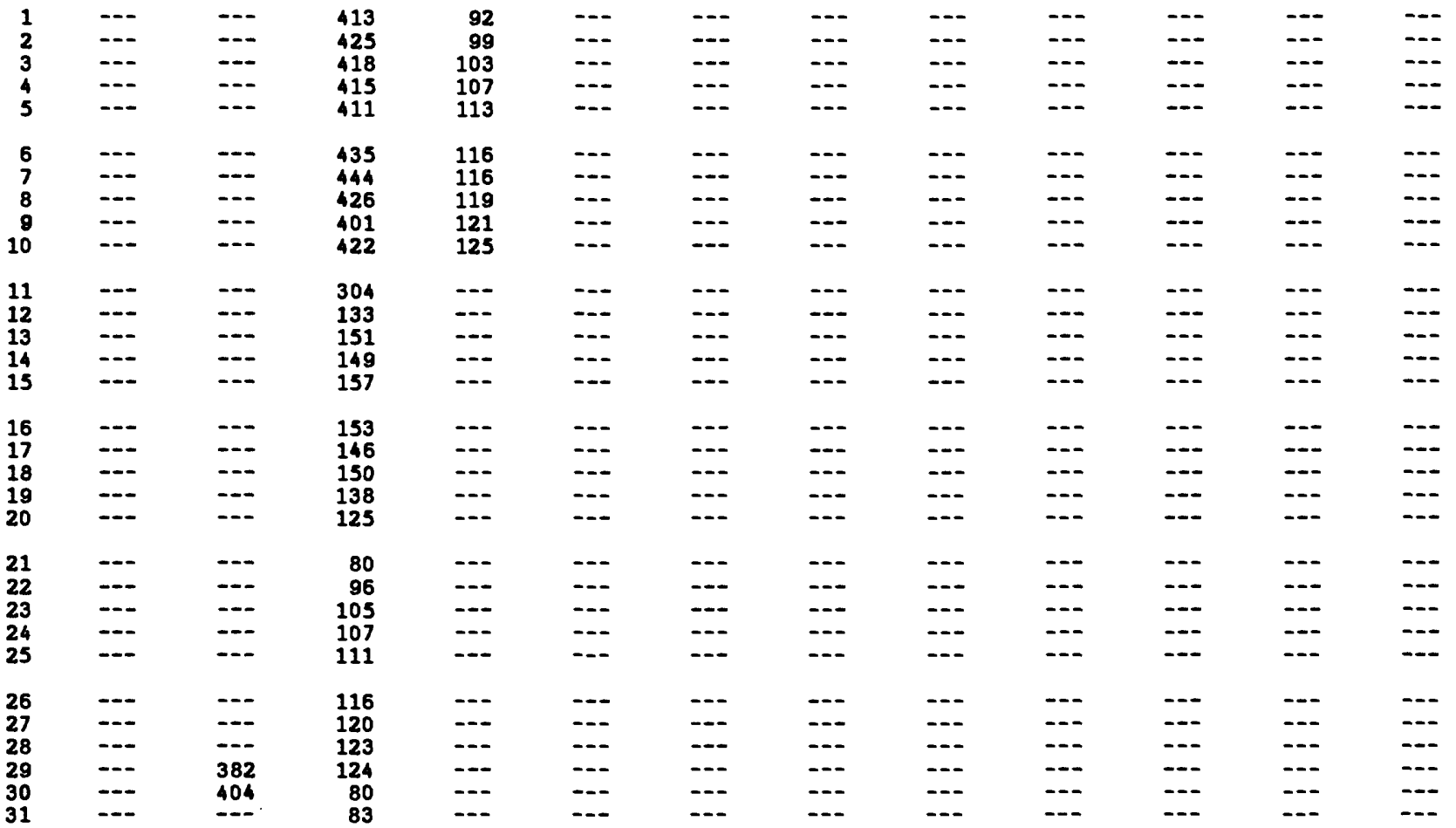


APPENDIX VI--Daily specific conductance--Continued

[Mean values in microsiemens per centimeter at 25 degrees Celsius]

\begin{tabular}{|c|c|c|c|c|c|c|c|c|c|c|c|c|}
\hline Day & Oct & Hov & Dec & Jan & $\overline{F e b}$ & Mar & $\overline{\text { Apr }}$ & May & June & July & Aug & Sept \\
\hline \multicolumn{13}{|c|}{$\begin{array}{l}\text { Site 3--Williamson Boulevard Ditch at Daytona Beach } \\
\text { [Water year October } 1983 \text { to September 1984] }\end{array}$} \\
\hline 1 & -- & --- & --- & --- & --- & --- & --- & --- & --- & --- & --- & 464 \\
\hline 2 & -- & -- & -- & -- & -- & -- & --- & -- & --- & -- & -- & 481 \\
\hline 3 & -- & --- & --- & --- & -- & -- & -- & -- & --- & --- & --- & 508 \\
\hline 4 & -- & -- & -- & -- & -- & --- & -- & -- & -- & -- & -- & 510 \\
\hline 5 & -- & --- & -- & --- & -- & -- & -- & -- & -- & -- & -- & 480 \\
\hline 6 & -- & -- & -- & -- & -- & --- & --- & -- & -- & -- & -- & 486 \\
\hline 7 & --- & --- & --- & --- & --- & --- & -- & -- & --- & --- & --- & 490 \\
\hline 8 & -- & -- & -- & -- & -- & --- & -- & -- & -- & -- & -- & 469 \\
\hline 9 & --- & -- & -- & -- & -- & --- & -- & -- & --- & -- & --- & 465 \\
\hline 10 & $\cdots$ & -- & -- & -- & -- & -- & -- & $\cdots$ & -- & -- & -- & 316 \\
\hline 11 & --- & -- & --- & -- & --- & --- & -- & -- & --- & -- & -- & 259 \\
\hline 12 & --- & -- & -- & -- & -- & --- & -- & -- & -- & -- & --- & 270 \\
\hline 13 & -- & -- & --- & --- & -- & -- & --- & -- & --- & --- & --- & 311 \\
\hline 14 & --- & -- & --- & -- & -- & --- & -- & -- & -- & -- & --- & 366 \\
\hline 15 & -- & --- & -- & --- & -- & --- & -- & -- & -- & -- & --- & 380 \\
\hline 16 & --- & --- & --- & --- & --- & --- & -- & -- & --- & --- & 532 & 397 \\
\hline 17 & -- & --- & -- & -- & - & --- & - & - & --- & -- & 547 & 412 \\
\hline 18 & -- & $-\cdot$ & -- & -- & -- & --- & -- & -- & -- & -- & 808 & 424 \\
\hline 19 & --- & -- & -- & -- & -- & --- & -- & -- & -- & -- & 865 & 408 \\
\hline 20 & -- & -- & --- & --- & --- & --- & -- & -- & -- & --- & 713 & 397 \\
\hline 21 & -- & -- & --- & -- & -- & -- & -- & -- & --- & -- & 487 & 377 \\
\hline 22 & --- & --- & --- & --- & --- & -- & --- & -- & --- & --- & 422 & 374 \\
\hline 23 & -- & -- & -- & -- & -- & --- & -- & -- & -- & --- & 422 & 382 \\
\hline 24 & -- & --- & --- & --- & -- & --- & -- & -- & -- & --- & 433 & 415 \\
\hline 25 & -- & -- & -- & -- & -- & --- & --- & --- & -- & -- & 433 & 502 \\
\hline 26 & -- & -- & --- & --- & --- & --- & --- & --- & -- & $=--$ & 448 & \\
\hline 27 & -- & --- & --- & --- & --- & -- & -- & --- & --- & -- & 404 & 486 \\
\hline 28 & -- & -- & -- & --- & --- & -- & -- & -- & -- & -- & 406 & 246 \\
\hline 29 & --- & --- & --- & --- & --- & --- & -- & --- & --- & --- & 426 & 182 \\
\hline 30 & -- & -- & -- & -- & -- & --- & -- & -- & -- & $\cdots$ & 458 & 185 \\
\hline 31 & --- & --- & --- & --- & -- & -- & $\ldots$ & -- & -- & -- & 449 & - \\
\hline
\end{tabular}

Site 4--Wally Hoffmeyer Canal at Daytona Beach [Water year October 1984 to September 1985]

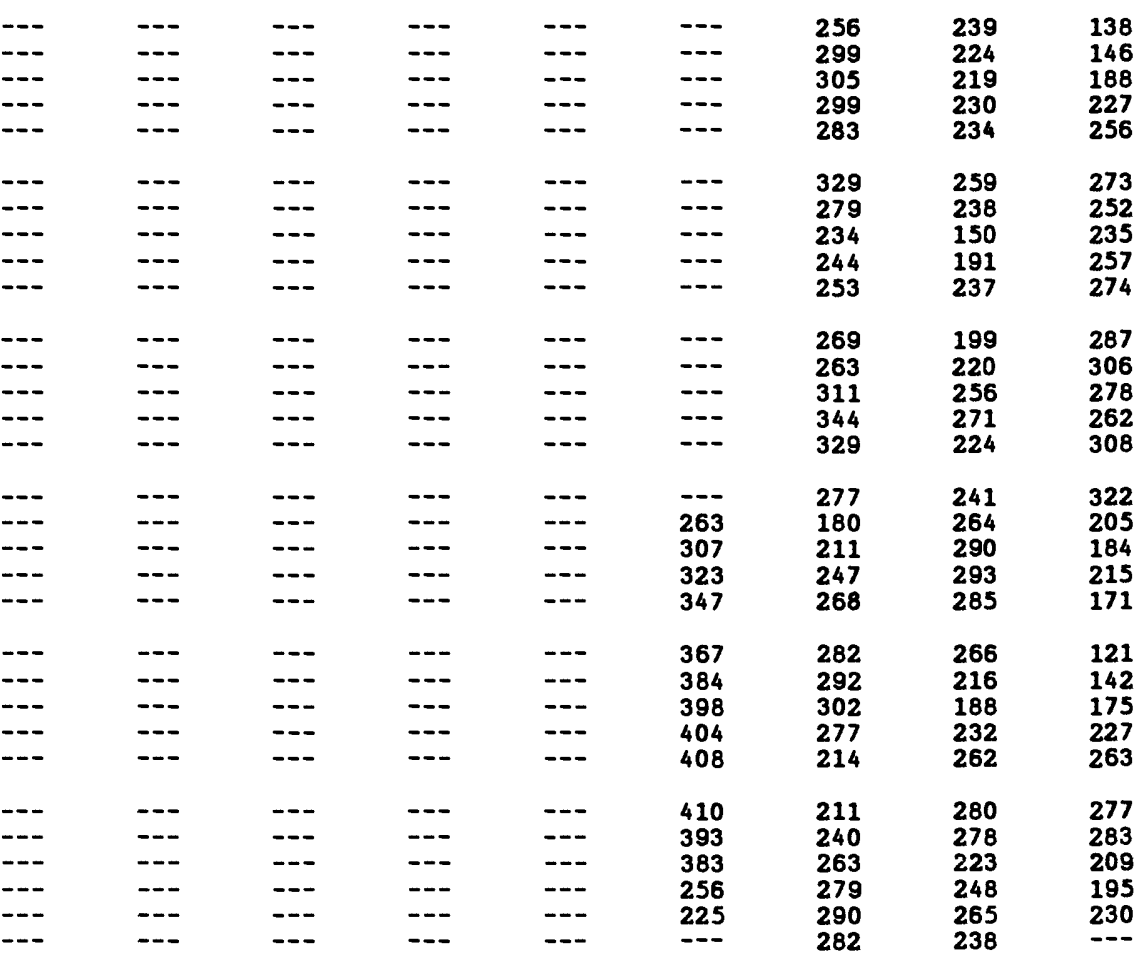


APPENDIX VI--Daily specific conductance--Continued

[Mean values in microsiemens per centimeter at 25 degrees Celsius]

\begin{tabular}{|c|c|c|c|c|c|c|c|c|c|c|c|c|}
\hline$\overline{\text { Day }}$ & Oct & Nov & $\overline{D e c}$ & $\operatorname{Jan}$ & Fab & Mar & Apr & May & June & July & Aug & Sept \\
\hline & \multicolumn{12}{|c|}{$\begin{array}{l}\text { Site 6--Bayless Boulevard Canal at Daytona Beach } \\
\text { [Water year October } 1983 \text { to September 1984] }\end{array}$} \\
\hline $\begin{array}{l}1 \\
2 \\
3 \\
4 \\
5\end{array}$ & $\begin{array}{l}-- \\
--- \\
--- \\
--- \\
---\end{array}$ & $\begin{array}{l}\cdots- \\
\cdots- \\
\cdots- \\
\cdots-\end{array}$ & $\begin{array}{l}427 \\
340 \\
355 \\
437 \\
467\end{array}$ & $\begin{array}{l}266 \\
283 \\
296 \\
309 \\
322\end{array}$ & $\begin{array}{l}458 \\
464 \\
471 \\
329 \\
-\end{array}$ & $\begin{array}{l}335 \\
370 \\
403 \\
417 \\
407\end{array}$ & $\begin{array}{l}-- \\
-- \\
--- \\
-- \\
--\end{array}$ & $\begin{array}{l}--- \\
-- \\
-- \\
--\end{array}$ & $\begin{array}{l}-- \\
--- \\
--- \\
--- \\
---\end{array}$ & $\begin{array}{l}--- \\
-\cdots \\
-\cdots \\
--\end{array}$ & $\overline{--}$ & $\begin{array}{l}\ldots \\
\ldots \\
\cdots \\
\cdots\end{array}$ \\
\hline $\begin{array}{r}6 \\
7 \\
8 \\
9 \\
10\end{array}$ & $\begin{array}{l}-- \\
-- \\
-- \\
-- \\
--\end{array}$ & $\overline{--}$ & $\begin{array}{l}481 \\
485 \\
484 \\
485 \\
493\end{array}$ & $\begin{array}{l}334 \\
347 \\
359 \\
372 \\
355\end{array}$ & $\begin{array}{l}--- \\
--- \\
492 \\
492 \\
494\end{array}$ & $\begin{array}{l}431 \\
451 \\
461 \\
464 \\
-\end{array}$ & $\begin{array}{l}-- \\
-- \\
--- \\
--- \\
--\end{array}$ & $\overline{--}$ & $\begin{array}{l}-- \\
--- \\
--- \\
---\end{array}$ & $\begin{array}{l}-\ldots \\
\ldots- \\
\ldots- \\
\ldots-\end{array}$ & $\overline{--}$ & $\begin{array}{l}-\cdots \\
-- \\
-\cdots \\
-\cdots\end{array}$ \\
\hline $\begin{array}{l}11 \\
12 \\
13 \\
14 \\
15\end{array}$ & $\begin{array}{l}-- \\
--- \\
--- \\
-- \\
--\end{array}$ & $\begin{array}{l}-\cdots \\
--- \\
--- \\
--- \\
--\end{array}$ & $\begin{array}{l}461 \\
105 \\
190 \\
245 \\
283\end{array}$ & $\begin{array}{l}283 \\
268 \\
328 \\
397 \\
416\end{array}$ & $\begin{array}{l}495 \\
492 \\
371 \\
233 \\
264\end{array}$ & $\begin{array}{l}-- \\
-\infty \\
-- \\
-- \\
-\infty\end{array}$ & $\begin{array}{l}-- \\
-- \\
-- \\
-- \\
--\end{array}$ & -- & $\begin{array}{l}-- \\
-- \\
-- \\
--- \\
--\end{array}$ & $\begin{array}{l}--- \\
-- \\
-\cdots \\
--\end{array}$ & $\ldots$ & $\begin{array}{l}--- \\
-- \\
-\ldots \\
--\end{array}$ \\
\hline $\begin{array}{l}16 \\
17 \\
18 \\
19 \\
20\end{array}$ & $\begin{array}{l}-- \\
-- \\
-- \\
-- \\
--\end{array}$ & $\begin{array}{c}--- \\
--- \\
-\cdots \\
-\cdots\end{array}$ & $\begin{array}{l}281 \\
302 \\
273 \\
263 \\
195\end{array}$ & $\begin{array}{l}427 \\
439 \\
434 \\
386 \\
287\end{array}$ & $\begin{array}{l}326 \\
367 \\
398 \\
422 \\
439\end{array}$ & $\begin{array}{l}--- \\
--- \\
--- \\
--- \\
---\end{array}$ & $\begin{array}{l}-- \\
-- \\
-- \\
-- \\
--\end{array}$ & $\begin{array}{l}--- \\
--- \\
--- \\
---\end{array}$ & $\begin{array}{l}-- \\
--- \\
-- \\
-- \\
---\end{array}$ & $\begin{array}{l}--- \\
--- \\
-\cdots \\
-\cdots\end{array}$ & $\begin{array}{l}--- \\
--- \\
\cdots- \\
---\end{array}$ & $\begin{array}{l}--- \\
-- \\
\cdots- \\
-\cdots \\
--\end{array}$ \\
\hline $\begin{array}{l}21 \\
22 \\
23 \\
24 \\
25\end{array}$ & $\begin{array}{l}--- \\
-- \\
--- \\
--- \\
---\end{array}$ & $\begin{array}{l}--- \\
267 \\
271 \\
345 \\
432\end{array}$ & $\begin{array}{l}150 \\
211 \\
247 \\
267 \\
288\end{array}$ & $\begin{array}{l}273 \\
276 \\
251 \\
254 \\
316\end{array}$ & $\begin{array}{l}443 \\
262 \\
229 \\
276 \\
327\end{array}$ & $\begin{array}{l}--- \\
--- \\
-- \\
-- \\
--\end{array}$ & $\begin{array}{l}-- \\
-- \\
-- \\
-- \\
--\end{array}$ & $\begin{array}{c}-\cdots \\
-\cdots \\
-\cdots\end{array}$ & $\begin{array}{l}-- \\
-- \\
-- \\
-- \\
--\end{array}$ & $\begin{array}{l}--- \\
-- \\
-- \\
--\end{array}$ & $\begin{array}{c}-\cdots \\
-\cdots \\
-\cdots\end{array}$ & $\begin{array}{l}--- \\
-- \\
-- \\
-- \\
--\end{array}$ \\
\hline $\begin{array}{l}26 \\
27 \\
28 \\
29 \\
30 \\
31\end{array}$ & $\begin{array}{l}\ldots \\
\cdots \\
\cdots \\
\cdots \\
\cdots\end{array}$ & $\begin{array}{l}450 \\
464 \\
485 \\
455 \\
435 \\
---\end{array}$ & $\begin{array}{l}305 \\
317 \\
323 \\
220 \\
202 \\
242\end{array}$ & $\begin{array}{l}393 \\
400 \\
411 \\
421 \\
434 \\
449\end{array}$ & $\begin{array}{l}370 \\
383 \\
334 \\
312 \\
--- \\
---\end{array}$ & $\begin{array}{l}--- \\
\cdots- \\
\cdots- \\
\cdots- \\
\cdots- \\
--\end{array}$ & $\begin{array}{l}--- \\
-\cdots \\
-- \\
-- \\
-\cdots\end{array}$ & 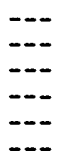 & $\begin{array}{l}--- \\
-\cdots \\
--- \\
-- \\
-\cdots \\
--\end{array}$ & $\begin{array}{l}--- \\
-- \\
--- \\
-- \\
--\end{array}$ & $\begin{array}{c}-\cdots \\
\cdots- \\
\cdots- \\
\cdots-\end{array}$ & $\begin{array}{c}--- \\
-\cdots \\
\cdots- \\
-\cdots \\
-\cdots\end{array}$ \\
\hline
\end{tabular}

Site 12--B-19 Canal at Dunlawton Avenue at Port Orange [Water year October 1982 to September 1983]

\begin{tabular}{|c|c|c|c|c|c|c|c|c|c|c|c|}
\hline $\begin{array}{l}-- \\
-- \\
-- \\
-- \\
--\end{array}$ & $\begin{array}{l}\cdots- \\
\cdots- \\
\cdots-\end{array}$ & $\begin{array}{l}-- \\
--- \\
-\cdots \\
---\end{array}$ & $\begin{array}{c}-- \\
-- \\
-- \\
-- \\
--\end{array}$ & $\begin{array}{l}\cdots- \\
\cdots- \\
-\cdots \\
-\cdots\end{array}$ & $\begin{array}{l}488 \\
--- \\
417 \\
455 \\
472\end{array}$ & $\begin{array}{l}496 \\
530 \\
527 \\
503 \\
471\end{array}$ & $\begin{array}{l}408 \\
424 \\
454 \\
488 \\
500\end{array}$ & $\begin{array}{l}243 \\
290 \\
320 \\
342 \\
286\end{array}$ & $\begin{array}{l}491 \\
459 \\
391 \\
453 \\
501\end{array}$ & $\begin{array}{l}526 \\
538 \\
527 \\
491 \\
446\end{array}$ & $\begin{array}{l}489 \\
404 \\
306 \\
326 \\
360\end{array}$ \\
\hline $\begin{array}{l}-- \\
-- \\
-- \\
-- \\
--\end{array}$ & $\begin{array}{l}\cdots- \\
-\cdots \\
-\cdots \\
--\end{array}$ & $\begin{array}{l}--- \\
--- \\
--- \\
---\end{array}$ & $\begin{array}{l}--- \\
-\cdots \\
-- \\
-- \\
--\end{array}$ & $\overline{-}$ & $\begin{array}{l}488 \\
478 \\
423 \\
408 \\
401\end{array}$ & $\begin{array}{l}448 \\
431 \\
429 \\
458 \\
417\end{array}$ & $\begin{array}{l}508 \\
517 \\
554 \\
594 \\
632\end{array}$ & $\begin{array}{l}223 \\
250 \\
227 \\
371 \\
375\end{array}$ & $\begin{array}{l}511 \\
503 \\
512 \\
525 \\
536\end{array}$ & $\begin{array}{l}495 \\
494 \\
481 \\
487 \\
382\end{array}$ & \begin{tabular}{l}
386 \\
\hdashline 424 \\
451 \\
457
\end{tabular} \\
\hline $\begin{array}{l}\cdots- \\
\cdots- \\
\cdots- \\
\cdots-\end{array}$ & 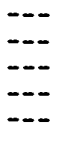 & $\begin{array}{l}--- \\
--- \\
--- \\
---\end{array}$ & $\begin{array}{l}-- \\
-- \\
-- \\
-- \\
--\end{array}$ & $\begin{array}{l}366 \\
371 \\
313 \\
267 \\
332\end{array}$ & $\begin{array}{l}403 \\
421 \\
434 \\
444 \\
427\end{array}$ & $\begin{array}{l}385 \\
423 \\
472 \\
450 \\
381\end{array}$ & $\begin{array}{l}567 \\
475 \\
481 \\
503 \\
500\end{array}$ & $\begin{array}{l}399 \\
415 \\
430 \\
436 \\
449\end{array}$ & $\begin{array}{l}539 \\
543 \\
547 \\
553 \\
555\end{array}$ & $\begin{array}{l}369 \\
440 \\
445 \\
353 \\
305\end{array}$ & $\begin{array}{l}465 \\
492 \\
505 \\
508 \\
509\end{array}$ \\
\hline $\begin{array}{l}-\cdots \\
-\cdots \\
-\cdots \\
-\cdots\end{array}$ & $\overline{--}$ & $\begin{array}{l}-- \\
-- \\
-- \\
-- \\
--\end{array}$ & $\begin{array}{l}--- \\
--- \\
--- \\
---\end{array}$ & $\begin{array}{l}380 \\
421 \\
449 \\
455 \\
475\end{array}$ & $\begin{array}{l}209 \\
201 \\
195 \\
210 \\
250\end{array}$ & $\begin{array}{l}199 \\
293 \\
315 \\
329 \\
261\end{array}$ & $\begin{array}{l}507 \\
516 \\
517 \\
514 \\
526\end{array}$ & $\begin{array}{l}462 \\
477 \\
491 \\
500 \\
506\end{array}$ & $\begin{array}{l}558 \\
569 \\
561 \\
548 \\
--\end{array}$ & $\begin{array}{l}320 \\
429 \\
653 \\
708 \\
713\end{array}$ & $\begin{array}{l}512 \\
427 \\
361 \\
353 \\
364\end{array}$ \\
\hline $\begin{array}{l}--- \\
--- \\
--- \\
---\end{array}$ & $\begin{array}{l}\cdots- \\
\cdots- \\
\cdots- \\
\cdots-\end{array}$ & $\begin{array}{l}--- \\
-\cdots \\
-- \\
--\end{array}$ & $\begin{array}{l}--- \\
--- \\
--- \\
---\end{array}$ & $\begin{array}{l}500 \\
553 \\
614 \\
646 \\
545\end{array}$ & $\begin{array}{l}262 \\
261 \\
284 \\
298 \\
326\end{array}$ & $\begin{array}{l}287 \\
333 \\
367 \\
249 \\
276\end{array}$ & $\begin{array}{l}531 \\
524 \\
524 \\
528 \\
529\end{array}$ & $\begin{array}{l}459 \\
302 \\
370 \\
414 \\
429\end{array}$ & $\begin{array}{l}549 \\
524 \\
574 \\
579 \\
585\end{array}$ & $\begin{array}{l}705 \\
687 \\
666 \\
--2 \\
417\end{array}$ & $\begin{array}{l}377 \\
384 \\
398 \\
420 \\
398\end{array}$ \\
\hline $\begin{array}{l}--- \\
--- \\
--- \\
--- \\
--- \\
---\end{array}$ & $\begin{array}{c}--- \\
--- \\
--- \\
-\cdots \\
--\end{array}$ & $\begin{array}{c}--- \\
-\cdots \\
-\cdots \\
\cdots- \\
-\cdots\end{array}$ & $\begin{array}{c}--- \\
-- \\
\cdots- \\
\cdots- \\
-\cdots\end{array}$ & $\begin{array}{l}437 \\
443 \\
469 \\
--- \\
--- \\
---\end{array}$ & $\begin{array}{l}369 \\
391 \\
401 \\
408 \\
426 \\
468\end{array}$ & $\begin{array}{l}380 \\
404 \\
386 \\
391 \\
400 \\
\ldots\end{array}$ & $\begin{array}{l}522 \\
531 \\
528 \\
479 \\
325 \\
259\end{array}$ & $\begin{array}{l}436 \\
448 \\
457 \\
471 \\
482 \\
-\end{array}$ & $\begin{array}{l}523 \\
450 \\
525 \\
447 \\
518 \\
493\end{array}$ & $\begin{array}{l}440 \\
451 \\
460 \\
470 \\
488 \\
494\end{array}$ & $\begin{array}{l}391 \\
405 \\
431 \\
435 \\
443 \\
-\end{array}$ \\
\hline
\end{tabular}


APPENDIX VI--Daily specific conductance--Continued

[Mean values in microsiemens per centimeter at 25 degrees Celsius]

\begin{tabular}{|c|c|c|c|c|c|c|c|c|c|c|c|c|}
\hline Day & Oct & Nov & Dec & Jan & $F \otimes b$ & Mar & Apr & May & June & July & Aug & Sept \\
\hline \multicolumn{13}{|c|}{$\begin{array}{l}\text { Site 12--B-19 Canal at Dunlawton Avenue at Port Orange } \\
\text { [Water year October } 1983 \text { to September 1984] }\end{array}$} \\
\hline $\begin{array}{l}1 \\
2 \\
3 \\
4 \\
5\end{array}$ & $\begin{array}{l}452 \\
462 \\
474 \\
479 \\
598\end{array}$ & $\begin{array}{l}431 \\
437 \\
448 \\
455 \\
448\end{array}$ & $\begin{array}{l}464 \\
452 \\
474 \\
483 \\
472\end{array}$ & $\begin{array}{l}--- \\
--- \\
--- \\
---\end{array}$ & $\begin{array}{l}--- \\
--- \\
--- \\
---\end{array}$ & $\begin{array}{l}=-- \\
=-- \\
=-- \\
---\end{array}$ & $\begin{array}{l}--- \\
-=- \\
--- \\
--\end{array}$ & $\begin{array}{l}577 \\
583 \\
581 \\
573 \\
495\end{array}$ & $\begin{array}{l}--- \\
--- \\
--- \\
---\end{array}$ & $\begin{array}{l}539 \\
496 \\
445 \\
402 \\
381\end{array}$ & $\begin{array}{l}=-- \\
=-- \\
=-- \\
---\end{array}$ & $\begin{array}{l}464 \\
481 \\
508 \\
510 \\
480\end{array}$ \\
\hline $\begin{array}{r}6 \\
7 \\
8 \\
9 \\
10\end{array}$ & $\begin{array}{l}479 \\
491 \\
487 \\
430 \\
414\end{array}$ & $\begin{array}{l}453 \\
451 \\
471 \\
453 \\
455\end{array}$ & $\begin{array}{l}463 \\
481 \\
493 \\
471 \\
479\end{array}$ & $\begin{array}{l}--- \\
--- \\
--- \\
---\end{array}$ & $\begin{array}{l}--- \\
--- \\
491 \\
504 \\
513\end{array}$ & $\begin{array}{l}--- \\
--- \\
--- \\
--- \\
---\end{array}$ & $\begin{array}{l}--- \\
--- \\
--- \\
---\end{array}$ & $\begin{array}{l}508 \\
565 \\
560 \\
581 \\
571\end{array}$ & $\begin{array}{l}--- \\
--- \\
-556 \\
556\end{array}$ & $\begin{array}{l}373 \\
391 \\
401 \\
425 \\
443\end{array}$ & $\begin{array}{l}=-- \\
=-- \\
=-- \\
---\end{array}$ & $\begin{array}{l}486 \\
490 \\
469 \\
465 \\
316\end{array}$ \\
\hline $\begin{array}{l}11 \\
12 \\
13 \\
14 \\
15\end{array}$ & $\begin{array}{l}340 \\
261 \\
310 \\
311 \\
326\end{array}$ & $\begin{array}{l}474 \\
482 \\
491 \\
497 \\
501\end{array}$ & $\begin{array}{l}471 \\
176 \\
179 \\
222 \\
252\end{array}$ & $\begin{array}{l}--- \\
--- \\
--- \\
---\end{array}$ & $\begin{array}{l}517 \\
511 \\
500 \\
473 \\
498\end{array}$ & $\begin{array}{l}--- \\
--- \\
--- \\
--- \\
---\end{array}$ & $\begin{array}{l}--- \\
-- \\
437 \\
412 \\
364\end{array}$ & $\begin{array}{l}557 \\
587 \\
593 \\
603 \\
606\end{array}$ & $\begin{array}{l}558 \\
558 \\
558 \\
553 \\
561\end{array}$ & $\begin{array}{l}479 \\
524 \\
513 \\
530 \\
531\end{array}$ & $\begin{array}{l}=-- \\
=-- \\
--- \\
---\end{array}$ & $\begin{array}{l}259 \\
270 \\
311 \\
366 \\
380\end{array}$ \\
\hline $\begin{array}{l}16 \\
17 \\
18 \\
19 \\
20\end{array}$ & $\begin{array}{l}189 \\
217 \\
207 \\
249 \\
296\end{array}$ & $\begin{array}{l}447 \\
441 \\
427 \\
466 \\
415\end{array}$ & $\begin{array}{l}275 \\
299 \\
276 \\
260 \\
234\end{array}$ & $\begin{array}{l}--- \\
--- \\
--- \\
---\end{array}$ & $\begin{array}{l}\mathbf{5 2 3} \\
\mathbf{5 1 8} \\
\mathbf{5 1 2} \\
\mathbf{5 8 2} \\
\mathbf{6 2 6}\end{array}$ & $\begin{array}{l}--- \\
--- \\
--- \\
---\end{array}$ & $\begin{array}{l}391 \\
414 \\
436 \\
475 \\
492\end{array}$ & $\begin{array}{l}604 \\
599 \\
605 \\
639 \\
634\end{array}$ & $\begin{array}{l}570 \\
578 \\
572 \\
568 \\
571\end{array}$ & $\begin{array}{l}545 \\
540 \\
566 \\
518 \\
506\end{array}$ & $\begin{array}{l}532 \\
547 \\
808 \\
865 \\
713\end{array}$ & $\begin{array}{l}397 \\
412 \\
424 \\
408 \\
397\end{array}$ \\
\hline $\begin{array}{l}21 \\
22 \\
23 \\
24 \\
25\end{array}$ & $\begin{array}{l}338 \\
330 \\
343 \\
319 \\
316\end{array}$ & $\begin{array}{l}403 \\
425 \\
453 \\
452 \\
455\end{array}$ & $\begin{array}{l}144 \\
150 \\
197 \\
222 \\
245\end{array}$ & $\begin{array}{l}--- \\
--- \\
--- \\
---\end{array}$ & $\begin{array}{l}626 \\
--- \\
--- \\
---\end{array}$ & $\begin{array}{l}-- \\
--- \\
--- \\
---\end{array}$ & $\begin{array}{l}503 \\
505 \\
513 \\
537 \\
574\end{array}$ & $\begin{array}{l}659 \\
627 \\
522 \\
352 \\
347\end{array}$ & $\begin{array}{l}578 \\
610 \\
458 \\
473 \\
483\end{array}$ & $\begin{array}{l}501 \\
473 \\
445 \\
437 \\
441\end{array}$ & $\begin{array}{l}487 \\
422 \\
422 \\
433 \\
433\end{array}$ & $\begin{array}{l}377 \\
374 \\
382 \\
415 \\
502\end{array}$ \\
\hline $\begin{array}{l}26 \\
27 \\
28 \\
29 \\
30 \\
31\end{array}$ & $\begin{array}{l}368 \\
382 \\
396 \\
411 \\
415\end{array}$ & $\begin{array}{l}462 \\
472 \\
480 \\
458 \\
451 \\
---\end{array}$ & $\begin{array}{l}314 \\
336 \\
349 \\
196 \\
--- \\
---\end{array}$ & $\begin{array}{l}--- \\
=- \\
=- \\
-- \\
--\end{array}$ & $\begin{array}{l}--- \\
--- \\
--- \\
--- \\
--\end{array}$ & $\begin{array}{l}--- \\
=-- \\
--- \\
--- \\
---\end{array}$ & $\begin{array}{l}592 \\
587 \\
587 \\
585 \\
591 \\
---\end{array}$ & $\begin{array}{l}358 \\
351 \\
352 \\
369 \\
380 \\
-\end{array}$ & $\begin{array}{l}437 \\
485 \\
501 \\
526 \\
563 \\
-\end{array}$ & $\begin{array}{l}458 \\
472 \\
484 \\
493 \\
503 \\
---\end{array}$ & $\begin{array}{l}448 \\
404 \\
406 \\
426 \\
458 \\
449\end{array}$ & $\begin{array}{l}458 \\
486 \\
246 \\
182 \\
185 \\
---\end{array}$ \\
\hline
\end{tabular}

Site 12--B-19 Canal at Dunlawton Avenue at Port Orange [Water year October 1984 to September 1985]

\begin{tabular}{|c|c|c|c|c|c|c|c|c|c|c|c|c|}
\hline $\begin{array}{l}1 \\
2 \\
3 \\
4 \\
5\end{array}$ & $\begin{array}{l}209 \\
237 \\
252 \\
261 \\
270\end{array}$ & $\begin{array}{l}373 \\
370 \\
367 \\
367 \\
355\end{array}$ & \begin{tabular}{l}
323 \\
322 \\
323 \\
\hdashline 408
\end{tabular} & $\begin{array}{l}586 \\
544 \\
512 \\
-20 \\
523\end{array}$ & $\begin{array}{l}583 \\
569 \\
587 \\
620 \\
594\end{array}$ & $\begin{array}{l}587 \\
595 \\
593 \\
644 \\
670\end{array}$ & $\begin{array}{l}615 \\
626 \\
617 \\
610 \\
630\end{array}$ & $\begin{array}{l}629 \\
648 \\
629 \\
657 \\
661\end{array}$ & $\begin{array}{l}581 \\
688 \\
582 \\
605 \\
589\end{array}$ & $\begin{array}{l}476 \\
477 \\
473 \\
477 \\
489\end{array}$ & $\begin{array}{l}703 \\
624 \\
567 \\
420 \\
433\end{array}$ & $\begin{array}{l}--- \\
--- \\
-- \\
-- \\
--\end{array}$ \\
\hline $\begin{array}{r}6 \\
7 \\
8 \\
9 \\
10\end{array}$ & $\begin{array}{l}281 \\
284 \\
301 \\
315 \\
315\end{array}$ & $\begin{array}{l}344 \\
349 \\
348 \\
351 \\
362\end{array}$ & $\begin{array}{l}402 \\
411 \\
436 \\
434 \\
426\end{array}$ & $\begin{array}{l}520 \\
542 \\
550 \\
541 \\
557\end{array}$ & $\begin{array}{l}607 \\
608 \\
588 \\
571 \\
569\end{array}$ & $\begin{array}{l}666 \\
656 \\
677 \\
666 \\
667\end{array}$ & $\begin{array}{l}635 \\
637 \\
614 \\
574 \\
596\end{array}$ & $\begin{array}{l}635 \\
633 \\
629 \\
598 \\
630\end{array}$ & $\begin{array}{l}619 \\
634 \\
610 \\
587 \\
609\end{array}$ & $\begin{array}{l}466 \\
472 \\
471 \\
471 \\
484\end{array}$ & $\begin{array}{l}410 \\
399 \\
372 \\
371 \\
347\end{array}$ & $\begin{array}{l}--- \\
--- \\
-- \\
--- \\
--\end{array}$ \\
\hline $\begin{array}{l}11 \\
12 \\
13 \\
14 \\
15\end{array}$ & $\begin{array}{l}314 \\
335 \\
334 \\
333 \\
328\end{array}$ & $\begin{array}{l}358 \\
355 \\
354 \\
354 \\
353\end{array}$ & $\begin{array}{l}444 \\
438 \\
440 \\
442 \\
455\end{array}$ & $\begin{array}{l}576 \\
574 \\
558 \\
586 \\
563\end{array}$ & $\begin{array}{l}548 \\
552 \\
574 \\
564 \\
550\end{array}$ & $\begin{array}{l}646 \\
652 \\
655 \\
642 \\
649\end{array}$ & $\begin{array}{l}576 \\
577 \\
574 \\
\ldots- \\
\ldots\end{array}$ & $\begin{array}{l}584 \\
583 \\
597 \\
612 \\
641\end{array}$ & $\begin{array}{l}620 \\
647 \\
585 \\
539 \\
447\end{array}$ & $\begin{array}{l}493 \\
496 \\
491 \\
503 \\
501\end{array}$ & $\begin{array}{l}329 \\
317 \\
300 \\
239 \\
224\end{array}$ & $\begin{array}{l}--- \\
--- \\
--- \\
---\end{array}$ \\
\hline $\begin{array}{l}16 \\
17 \\
18 \\
19 \\
20\end{array}$ & $\begin{array}{l}330 \\
340 \\
350 \\
340 \\
355\end{array}$ & $\begin{array}{l}351 \\
347 \\
353 \\
354 \\
378\end{array}$ & $\begin{array}{l}450 \\
446 \\
471 \\
472 \\
462\end{array}$ & $\begin{array}{l}577 \\
558 \\
594 \\
573 \\
585\end{array}$ & $\begin{array}{l}544 \\
566 \\
548 \\
550 \\
572\end{array}$ & $\begin{array}{l}655 \\
650 \\
624 \\
617 \\
633\end{array}$ & $\begin{array}{l}--- \\
--- \\
--- \\
--- \\
---\end{array}$ & $\begin{array}{l}626 \\
630 \\
624 \\
593 \\
607\end{array}$ & $\begin{array}{l}439 \\
439 \\
401 \\
--- \\
---\end{array}$ & $\begin{array}{l}496 \\
498 \\
504 \\
509 \\
505\end{array}$ & $\begin{array}{l}254 \\
284 \\
319 \\
350 \\
326\end{array}$ & $\begin{array}{l}-- \\
--- \\
-- \\
--- \\
--\end{array}$ \\
\hline $\begin{array}{l}21 \\
22 \\
23 \\
24 \\
25\end{array}$ & $\begin{array}{l}360 \\
372 \\
390 \\
454 \\
477\end{array}$ & $\begin{array}{l}364 \\
367 \\
368 \\
374 \\
374\end{array}$ & $\begin{array}{l}472 \\
486 \\
490 \\
495 \\
490\end{array}$ & $\begin{array}{l}558 \\
555 \\
552 \\
540 \\
556\end{array}$ & $\begin{array}{l}563 \\
563 \\
579 \\
574 \\
587\end{array}$ & $\begin{array}{l}622 \\
612 \\
639 \\
637 \\
605\end{array}$ & $\begin{array}{l}--- \\
--- \\
--- \\
--- \\
---\end{array}$ & $\begin{array}{l}603 \\
604 \\
580 \\
574 \\
560\end{array}$ & $\begin{array}{l}--- \\
--- \\
--2 \\
--- \\
493\end{array}$ & $\begin{array}{l}524 \\
514 \\
514 \\
525 \\
518\end{array}$ & $\begin{array}{l}-- \\
-- \\
--- \\
--\infty \\
--\infty\end{array}$ & $\begin{array}{l}--- \\
--- \\
--- \\
-- \\
--\end{array}$ \\
\hline $\begin{array}{l}26 \\
27 \\
28 \\
29 \\
30 \\
31\end{array}$ & $\begin{array}{l}530 \\
348 \\
338 \\
353 \\
360 \\
369\end{array}$ & $\begin{array}{l}353 \\
358 \\
345 \\
339 \\
323 \\
---\end{array}$ & $\begin{array}{l}482 \\
481 \\
497 \\
566 \\
571 \\
656\end{array}$ & $\begin{array}{l}565 \\
567 \\
573 \\
567 \\
567 \\
572\end{array}$ & $\begin{array}{l}579 \\
570 \\
571 \\
--- \\
--- \\
---\end{array}$ & $\begin{array}{l}619 \\
623 \\
627 \\
628 \\
626 \\
643\end{array}$ & $\begin{array}{l}-- \\
-- \\
-- \\
-- \\
-- \\
--\end{array}$ & $\begin{array}{l}551 \\
546 \\
538 \\
545 \\
575 \\
576\end{array}$ & $\begin{array}{l}484 \\
493 \\
494 \\
494 \\
478 \\
---\end{array}$ & $\begin{array}{l}514 \\
517 \\
510 \\
515 \\
521 \\
524\end{array}$ & $\begin{array}{l}-- \\
-- \\
--- \\
-- \\
-- \\
--\end{array}$ & $\begin{array}{l}--- \\
--- \\
--- \\
--- \\
-- \\
--\end{array}$ \\
\hline
\end{tabular}


Appendix VII 
APPENDIX VII--Daily dissolved oxysen

[Mean values, milligrams per liter]

\begin{tabular}{|c|c|c|c|c|c|c|c|c|c|c|c|c|}
\hline$\overline{\text { Day }}$ & Oct & Nov & Dec & Jan & $\overline{\text { Feb }}$ & Mar & $\overline{A p r}$ & May & June & July & Aug & Sept \\
\hline & \multicolumn{12}{|c|}{$\begin{array}{l}\text { Site 1--Tomoka River near Bolly Bill } \\
\text { [Water year October } 1982 \text { to September 1983] }\end{array}$} \\
\hline $\begin{array}{l}1 \\
2 \\
3 \\
4 \\
5\end{array}$ & $\begin{array}{l}--- \\
--- \\
--- \\
--- \\
---\end{array}$ & $\begin{array}{l}--- \\
--- \\
--- \\
--- \\
---\end{array}$ & $\begin{array}{l}--- \\
--- \\
--- \\
--- \\
---\end{array}$ & $\begin{array}{l}--- \\
--- \\
--- \\
--- \\
---\end{array}$ & $\begin{array}{l}--- \\
--- \\
--- \\
---\end{array}$ & $\begin{array}{l}7.0 \\
7.3 \\
7.0 \\
6.8 \\
6.4\end{array}$ & $\begin{array}{l}7.1 \\
7.1 \\
7.2 \\
7.4 \\
6.9\end{array}$ & $\begin{array}{l}5.9 \\
5.7 \\
5.4 \\
5.2 \\
5.9\end{array}$ & $\begin{array}{l}5.2 \\
4.7 \\
4.8 \\
4.6 \\
4.4\end{array}$ & $\begin{array}{l}4.3 \\
4.3 \\
4.2 \\
4.0 \\
3.9\end{array}$ & $\begin{array}{l}--- \\
--- \\
--- \\
--- \\
---\end{array}$ & $\begin{array}{l}4.2 \\
4.5 \\
4.9 \\
4.7 \\
4.4\end{array}$ \\
\hline $\begin{array}{r}6 \\
7 \\
8 \\
9 \\
10\end{array}$ & $\begin{array}{l}--- \\
--- \\
--- \\
--- \\
---\end{array}$ & $\begin{array}{l}--- \\
--- \\
--- \\
--- \\
---\end{array}$ & $\begin{array}{l}--- \\
--- \\
--- \\
--- \\
---\end{array}$ & $\begin{array}{l}--- \\
--- \\
--- \\
--- \\
---\end{array}$ & $\begin{array}{l}--- \\
--- \\
--- \\
---\end{array}$ & $\begin{array}{l}5.7 \\
5.4 \\
5.7 \\
6.1 \\
6.7\end{array}$ & $\begin{array}{l}6.3 \\
6.0 \\
5.5 \\
5.6 \\
5.9\end{array}$ & $\begin{array}{l}6.0 \\
5.9 \\
5.8 \\
5.5 \\
5.4\end{array}$ & $\begin{array}{c}4.6 \\
5.0 \\
-.-2 \\
4.9 \\
4.7\end{array}$ & $\begin{array}{l}3.9 \\
3.8 \\
3.8 \\
3.9 \\
4.1\end{array}$ & $\begin{array}{l}--- \\
--- \\
--- \\
6.8 \\
4.8\end{array}$ & $\begin{array}{l}4.2 \\
3.9 \\
3.9 \\
3.8\end{array}$ \\
\hline $\begin{array}{l}11 \\
12 \\
13 \\
14 \\
15\end{array}$ & $\begin{array}{l}-- \\
--- \\
--- \\
--- \\
---\end{array}$ & $\begin{array}{l}--- \\
--- \\
--- \\
--- \\
---\end{array}$ & $\begin{array}{l}--- \\
--- \\
--- \\
--- \\
---\end{array}$ & $\begin{array}{l}--- \\
--- \\
--- \\
--- \\
---\end{array}$ & $\begin{array}{l}--- \\
7.8 \\
7.9 \\
7.7 \\
8.0\end{array}$ & $\begin{array}{l}7.9 \\
8.5 \\
8.6 \\
8.6 \\
7.8\end{array}$ & $\begin{array}{l}6.3 \\
6.5 \\
6.6 \\
6.4 \\
6.3\end{array}$ & $\begin{array}{l}5.3 \\
5.2 \\
5.2 \\
5.3 \\
5.0\end{array}$ & $\begin{array}{l}4.7 \\
4.6 \\
4.7 \\
4.8 \\
4.8\end{array}$ & $\begin{array}{l}4.1 \\
4.2 \\
4.2 \\
7.1 \\
8.0\end{array}$ & $\begin{array}{l}4.5 \\
4.2 \\
4.6 \\
4.9 \\
5.0\end{array}$ & $\begin{array}{l}3.6 \\
3.6 \\
4.8 \\
7.8 \\
8.4\end{array}$ \\
\hline $\begin{array}{l}16 \\
17 \\
18 \\
19 \\
20\end{array}$ & $\begin{array}{l}--- \\
--- \\
--- \\
-- \\
---\end{array}$ & $\begin{array}{l}--- \\
--- \\
--- \\
--- \\
---\end{array}$ & $\begin{array}{l}--- \\
--- \\
--- \\
--- \\
---\end{array}$ & $\begin{array}{l}--- \\
--- \\
--- \\
--- \\
---\end{array}$ & $\begin{array}{l}7.8 \\
7.4 \\
7.6 \\
7.6 \\
7.4\end{array}$ & $\begin{array}{l}7.3 \\
6.7 \\
6.4 \\
6.6 \\
6.5\end{array}$ & $\begin{array}{l}6.7 \\
7.1 \\
7.4 \\
7.5 \\
7.6\end{array}$ & $\begin{array}{l}4.5 \\
4.4 \\
4.4 \\
4.5 \\
4.5\end{array}$ & $\begin{array}{l}4.9 \\
4.7 \\
4.8 \\
4.7 \\
4.4\end{array}$ & $\begin{array}{l}7.6 \\
7.1 \\
7.0 \\
7.0 \\
---\end{array}$ & $\begin{array}{l}4.9 \\
4.6 \\
4.6 \\
4.5 \\
4.3\end{array}$ & $\begin{array}{l}8.5 \\
7.0 \\
4.4 \\
4.5 \\
4.5\end{array}$ \\
\hline $\begin{array}{l}21 \\
22 \\
23 \\
24 \\
25\end{array}$ & $\begin{array}{l}--- \\
--- \\
--- \\
--- \\
---\end{array}$ & $\begin{array}{l}--- \\
--- \\
--- \\
-- \\
---\end{array}$ & $\begin{array}{l}--- \\
--- \\
--- \\
--- \\
---\end{array}$ & $\begin{array}{l}--- \\
--- \\
--- \\
--- \\
---\end{array}$ & $\begin{array}{l}7.1 \\
6.8 \\
6.5 \\
6.9 \\
7.0\end{array}$ & $\begin{array}{l}6.4 \\
7.1 \\
7.8 \\
7.5 \\
8.0\end{array}$ & $\begin{array}{l}7.8 \\
7.2 \\
6.2 \\
6.0 \\
6.2\end{array}$ & $\begin{array}{l}4.3 \\
4.3 \\
4.4 \\
6.0 \\
8.0\end{array}$ & $\begin{array}{l}4.1 \\
4.4 \\
4.6 \\
4.7 \\
4.7\end{array}$ & $\begin{array}{l}--- \\
--- \\
--- \\
--- \\
---\end{array}$ & $\begin{array}{l}4.2 \\
4.1 \\
4.1 \\
4.2 \\
4.3\end{array}$ & $\begin{array}{l}4.4 \\
4.4 \\
4.7 \\
4.6 \\
4.8\end{array}$ \\
\hline $\begin{array}{l}26 \\
27 \\
28 \\
29 \\
30 \\
31\end{array}$ & $\begin{array}{l}--- \\
--- \\
--- \\
--- \\
---\end{array}$ & $\begin{array}{l}--- \\
--- \\
--- \\
--- \\
--- \\
---\end{array}$ & $\begin{array}{l}--- \\
--- \\
--- \\
--- \\
--- \\
---\end{array}$ & $\begin{array}{l}--- \\
--- \\
--- \\
--- \\
---\end{array}$ & $\begin{array}{l}7.4 \\
7.8 \\
7.4 \\
--- \\
--- \\
---\end{array}$ & $\begin{array}{l}9.0 \\
8.9 \\
8.6 \\
9.1 \\
6.0 \\
8.2\end{array}$ & $\begin{array}{l}6.4 \\
6.5 \\
6.4 \\
6.2 \\
6.2 \\
---\end{array}$ & $\begin{array}{l}8.3 \\
9.0 \\
7.1 \\
6.5 \\
4.8 \\
5.3\end{array}$ & $\begin{array}{l}4.6 \\
4.5 \\
4.4 \\
4.2 \\
4.2 \\
-.-\end{array}$ & $\begin{array}{l}--- \\
--- \\
--- \\
--- \\
--- \\
---\end{array}$ & $\begin{array}{l}4.4 \\
4.4 \\
4.3 \\
4.2 \\
3.8 \\
4.0\end{array}$ & $\begin{array}{l}5.0 \\
4.8 \\
4.8 \\
4.8 \\
4.7 \\
-.-\end{array}$ \\
\hline
\end{tabular}

Site 1--Tomoka River near Bolly Bill

[Water year October 1983 to September 1984]

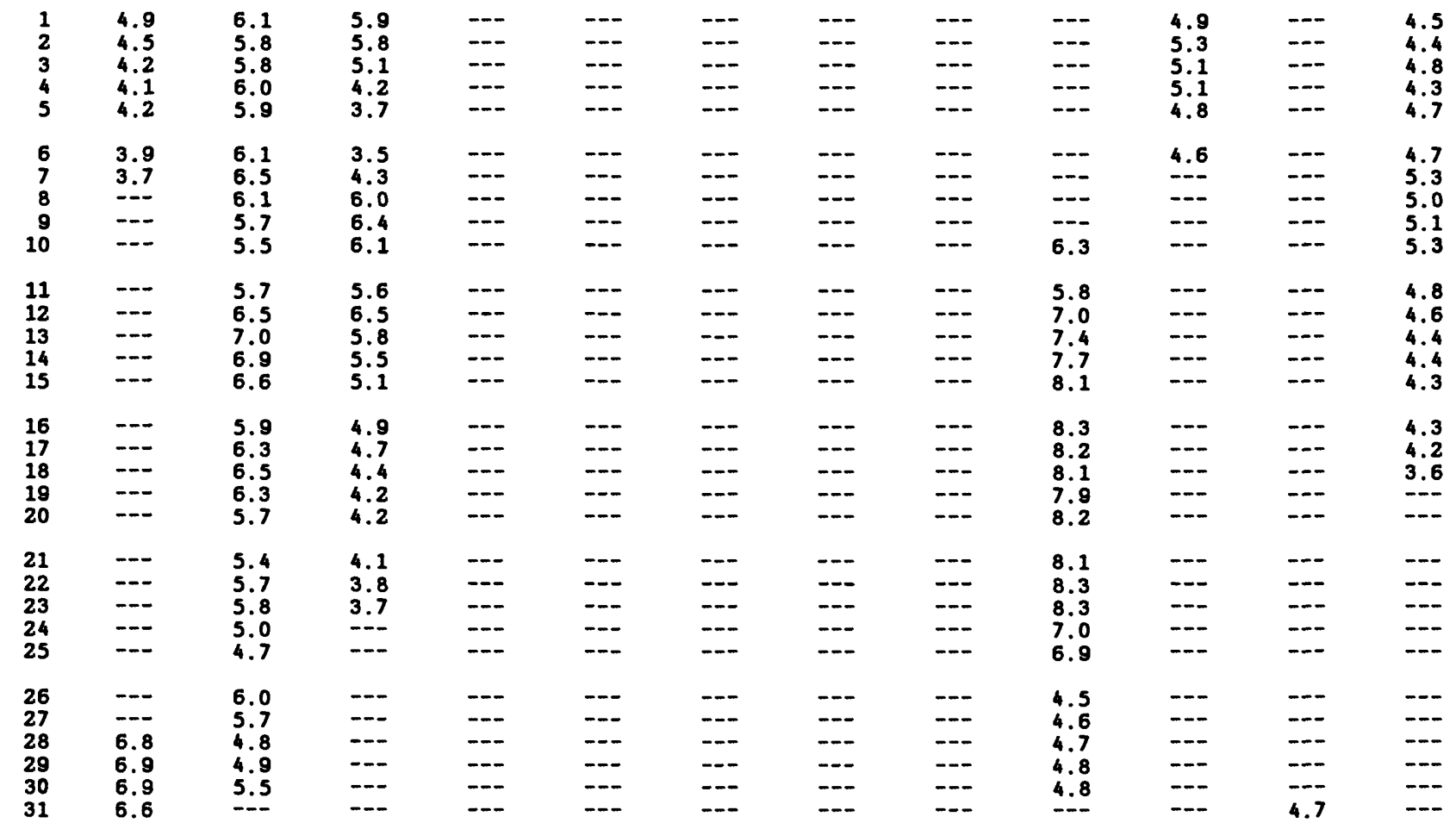


APPENDIX VII--Daily dissolved oxrgen--Continued

[Mean values, milligrams per liter]

\begin{tabular}{|c|c|c|c|c|c|c|c|c|c|c|c|c|}
\hline Day & Oct & Dov & Dec & Jan & Feb & Mar & Apr & May & June & July & Aug & Sept \\
\hline \multicolumn{13}{|c|}{$\begin{array}{l}\text { Site 12--B-19 Canal at Dunlawton Avenue near Port Orange } \\
\text { [Water year October } 1982 \text { to September 1983] }\end{array}$} \\
\hline $\begin{array}{l}1 \\
2 \\
3 \\
4 \\
5\end{array}$ & \begin{tabular}{l}
--- \\
-- \\
\hdashline-- \\
---
\end{tabular} & $\begin{array}{l}--- \\
-- \\
--- \\
---\end{array}$ & $\begin{array}{l}--- \\
\overline{-} \\
--- \\
---\end{array}$ & $\begin{array}{l}--- \\
--- \\
--- \\
---\end{array}$ & $\begin{array}{l}--- \\
--- \\
--- \\
---\end{array}$ & $\begin{array}{l}5.8 \\
6.7 \\
6.6 \\
6.5 \\
6.2\end{array}$ & $\begin{array}{l}6.2 \\
5.8 \\
6.0 \\
6.1 \\
5.7\end{array}$ & $\begin{array}{l}--- \\
--- \\
--- \\
---\end{array}$ & $\begin{array}{l}--- \\
--- \\
--- \\
---\end{array}$ & $\begin{array}{l}--- \\
--- \\
--- \\
--- \\
--\end{array}$ & $\begin{array}{l}--- \\
--- \\
--- \\
--\end{array}$ & $\begin{array}{l}--- \\
\overline{--} \\
\overline{--} \\
---\end{array}$ \\
\hline $\begin{array}{r}6 \\
7 \\
8 \\
9 \\
10\end{array}$ & $\begin{array}{l}--- \\
--- \\
-- \\
---\end{array}$ & $\begin{array}{l}--- \\
\overline{--} \\
\overline{--} \\
--\end{array}$ & $\begin{array}{l}--- \\
\overline{--} \\
\overline{--} \\
--\end{array}$ & $\begin{array}{l}--- \\
--- \\
--- \\
--- \\
--\end{array}$ & $\begin{array}{l}--- \\
--- \\
--- \\
---\end{array}$ & $\begin{array}{l}5.7 \\
5.5 \\
5.6 \\
5.9 \\
6.3\end{array}$ & $\begin{array}{l}5.4 \\
5.6 \\
5.2 \\
5.2 \\
5.6\end{array}$ & $\begin{array}{l}--- \\
--- \\
--- \\
--- \\
--\end{array}$ & $\begin{array}{l}--- \\
-- \\
--- \\
--- \\
---\end{array}$ & $\begin{array}{l}--- \\
--- \\
--- \\
--- \\
--\end{array}$ & $\begin{array}{l}-- \\
-- \\
-- \\
--\end{array}$ & $\begin{array}{l}-.- \\
5.1 \\
5.2 \\
5.2\end{array}$ \\
\hline $\begin{array}{l}11 \\
12 \\
13 \\
14 \\
15\end{array}$ & $\begin{array}{l}--- \\
--- \\
-\cdots \\
---\end{array}$ & $\begin{array}{l}--- \\
--- \\
--- \\
---\end{array}$ & $\begin{array}{l}--- \\
-- \\
--- \\
---\end{array}$ & $\begin{array}{l}--- \\
-\cdots \\
--- \\
---\end{array}$ & $\begin{array}{l}6.8 \\
6.4 \\
6.8 \\
6.1 \\
5.6\end{array}$ & $\begin{array}{l}7.1 \\
7.2 \\
7.2 \\
7.1 \\
6.5\end{array}$ & $\begin{array}{l}5.6 \\
5.3 \\
5.2 \\
5.3 \\
5.7\end{array}$ & $\begin{array}{l}--- \\
--- \\
--- \\
---\end{array}$ & $\begin{array}{l}--- \\
--- \\
--- \\
---\end{array}$ & \begin{tabular}{l}
--- \\
-- \\
\hdashline-- \\
---
\end{tabular} & $\begin{array}{l}-- \\
--- \\
--- \\
---\end{array}$ & $\begin{array}{l}5.2 \\
5.2 \\
5.2 \\
5.1 \\
5.3\end{array}$ \\
\hline $\begin{array}{l}16 \\
17 \\
18 \\
19 \\
20\end{array}$ & $\begin{array}{l}--- \\
-\cdots \\
-\cdots \\
---\end{array}$ & \begin{tabular}{c}
--- \\
--- \\
\hdashline-- \\
---
\end{tabular} & $\begin{array}{l}--- \\
-\cdots \\
-\cdots \\
-\cdots \\
---\end{array}$ & $\begin{array}{l}--- \\
--- \\
-\cdots \\
---\end{array}$ & $\begin{array}{l}5.5 \\
5.0 \\
5.2 \\
5.3 \\
5.2\end{array}$ & $\begin{array}{l}7.1 \\
6.5 \\
5.7 \\
5.4 \\
5.1\end{array}$ & $\begin{array}{l}5.8 \\
5.3 \\
5.3 \\
5.4 \\
---\end{array}$ & $\begin{array}{l}--- \\
--- \\
--- \\
-\cdots\end{array}$ & $\begin{array}{l}--- \\
--- \\
--- \\
---\end{array}$ & $\begin{array}{l}--- \\
--- \\
-\cdots \\
---\end{array}$ & $\begin{array}{l}-- \\
--- \\
--- \\
--\end{array}$ & $\begin{array}{l}5.6 \\
5.3 \\
5.2 \\
5.3 \\
5.1\end{array}$ \\
\hline $\begin{array}{l}21 \\
22 \\
23 \\
24 \\
25\end{array}$ & $\begin{array}{l}--- \\
--- \\
--- \\
--- \\
--\end{array}$ & $\begin{array}{l}--- \\
--- \\
--- \\
--- \\
---\end{array}$ & $\begin{array}{l}--- \\
--- \\
--- \\
--- \\
---\end{array}$ & $\begin{array}{l}--- \\
--- \\
--- \\
---\end{array}$ & $\begin{array}{l}4.9 \\
4.7 \\
4.7 \\
4.8 \\
5.4\end{array}$ & $\begin{array}{l}5.8 \\
6.3 \\
6.3 \\
6.2 \\
6.6\end{array}$ & $\begin{array}{l}--- \\
\overline{--} \\
\overline{--} \\
--\end{array}$ & $\begin{array}{l}--- \\
--- \\
--- \\
--- \\
--\end{array}$ & $\begin{array}{l}--- \\
--- \\
--- \\
---\end{array}$ & $\begin{array}{l}--- \\
--- \\
--- \\
--- \\
---\end{array}$ & $\begin{array}{l}--- \\
--- \\
--- \\
---\end{array}$ & $\begin{array}{l}5.0 \\
5.1 \\
5.3 \\
5.6 \\
5.6\end{array}$ \\
\hline $\begin{array}{l}26 \\
27 \\
28 \\
29 \\
30 \\
31\end{array}$ & $\begin{array}{l}--- \\
--- \\
--- \\
--- \\
--- \\
--\end{array}$ & $\begin{array}{l}--- \\
--- \\
--- \\
--- \\
--- \\
--\end{array}$ & $\begin{array}{l}--- \\
--- \\
--- \\
--- \\
---\end{array}$ & $\begin{array}{l}--- \\
--- \\
--- \\
--- \\
--- \\
--\end{array}$ & $\begin{array}{l}6.2 \\
6.1 \\
5.8 \\
--- \\
---\end{array}$ & $\begin{array}{l}6.4 \\
5.9 \\
5.9 \\
6.2 \\
6.3 \\
5.9\end{array}$ & $\begin{array}{l}--- \\
\overline{--} \\
--- \\
--- \\
---\end{array}$ & $\begin{array}{l}--- \\
--- \\
--- \\
--- \\
---\end{array}$ & $\begin{array}{l}--- \\
--- \\
--- \\
--- \\
---\end{array}$ & $\begin{array}{l}--- \\
--- \\
--- \\
--- \\
---\end{array}$ & $\begin{array}{l}--- \\
--- \\
--- \\
--- \\
--- \\
--\end{array}$ & $\begin{array}{l}5.4 \\
5.6 \\
5.4 \\
5.1 \\
5.1 \\
-\end{array}$ \\
\hline
\end{tabular}

Site 12--B-19 Canal at Dunlawton Avenue near Port Orange [Water year October 1983 to September 1984]

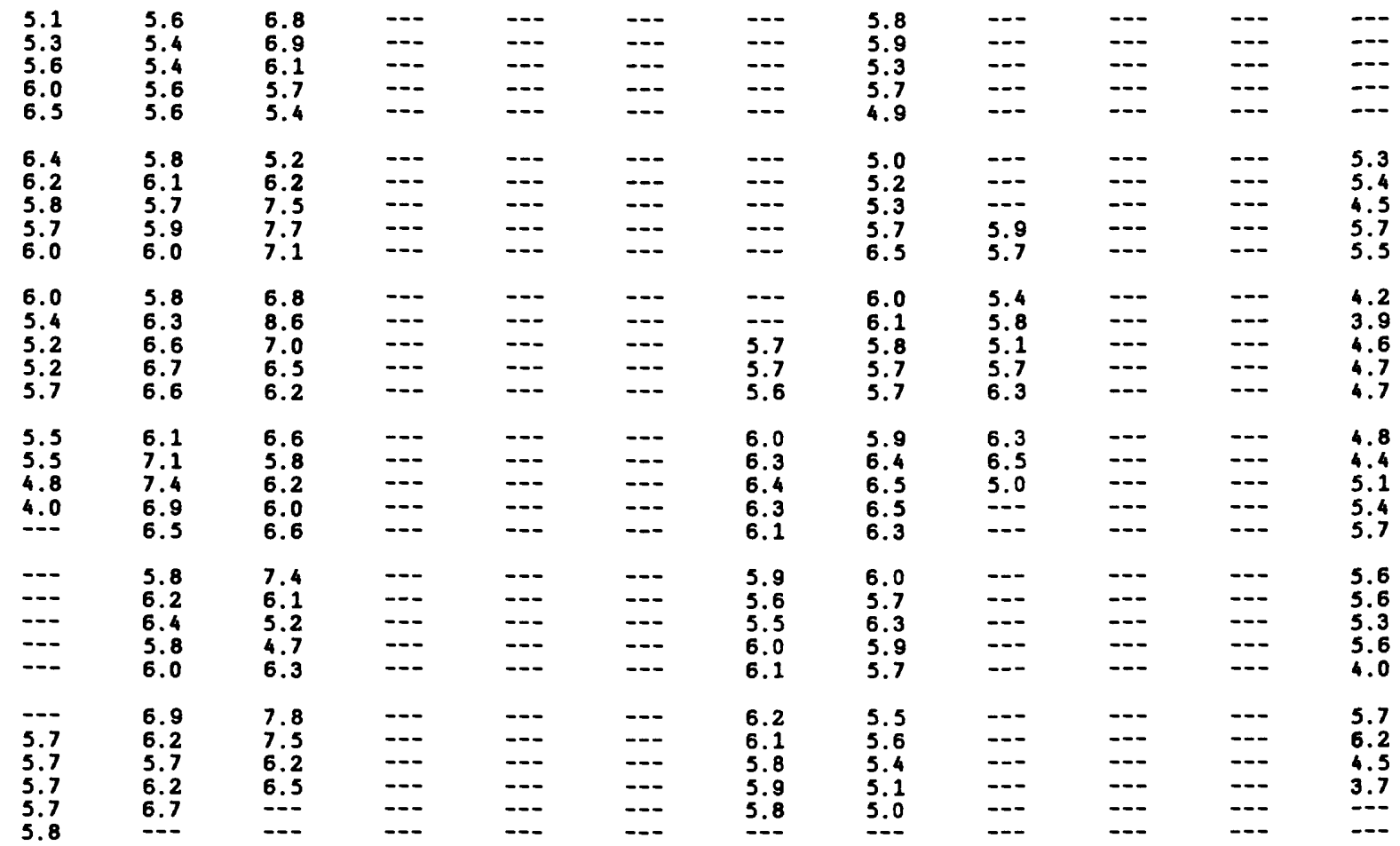


APPENDIX VII--Daily dissolved oxygen--Continued

[Mean values, milligrams per liter]

\begin{tabular}{|c|c|c|c|c|c|c|c|c|c|c|c|c|}
\hline Day & Oct & Nov & Dec & $\operatorname{Jan}$ & $F \bullet b$ & Mar & $\overline{\text { Apr }}$ & Thay & June & July & Aus & Sept \\
\hline \multicolumn{13}{|c|}{$\begin{array}{l}\text { Site 12--B-19 Canal at Dunlawton Avenue near Port Orange } \\
\text { [Water year October } 1984 \text { to September 1985] }\end{array}$} \\
\hline $\begin{array}{l}1 \\
2 \\
3 \\
4 \\
5\end{array}$ & $\begin{array}{l}-- \\
-\cdots \\
-\cdots \\
-\cdots\end{array}$ & \begin{tabular}{l}
-- \\
\hdashline- \\
\hdashline- \\
--
\end{tabular} & $\overline{-}$ & 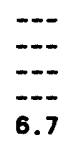 & $\begin{array}{l}6.0 \\
5.2 \\
5.1 \\
5.1 \\
5.7\end{array}$ & $\begin{array}{l}5.7 \\
5.6 \\
5.8 \\
5.6 \\
5.3\end{array}$ & $\begin{array}{l}4.4 \\
4.6 \\
4.8 \\
4.9 \\
4.8\end{array}$ & $\begin{array}{l}6.2 \\
8.1 \\
6.0 \\
5.6 \\
5.6\end{array}$ & $\begin{array}{l}\cdots \\
\cdots- \\
\cdots-\end{array}$ & $\begin{array}{l}4.8 \\
4.7 \\
4.7 \\
4.6 \\
4.5\end{array}$ & \begin{tabular}{l}
--- \\
\hdashline-- \\
\hdashline-
\end{tabular} & \begin{tabular}{l}
-- \\
-- \\
\hdashline- \\
--
\end{tabular} \\
\hline $\begin{array}{r}6 \\
7 \\
8 \\
9 \\
10\end{array}$ & $\begin{array}{l}--- \\
\overline{--} \\
\overline{--} \\
--\end{array}$ & $\begin{array}{l}--- \\
--- \\
--- \\
--\end{array}$ & $\begin{array}{l}5.0 \\
6.2 \\
6.6 \\
6.5 \\
6.6\end{array}$ & $\begin{array}{l}7.0 \\
7.2 \\
6.9 \\
7.1 \\
6.9\end{array}$ & \begin{tabular}{l}
5.2 \\
6.0 \\
\hdashline.- \\
.-
\end{tabular} & $\begin{array}{l}5.3 \\
5.5 \\
5.3 \\
5.3 \\
5.7\end{array}$ & $\begin{array}{l}4.5 \\
4.7 \\
4.8 \\
4.6 \\
4.7\end{array}$ & $\begin{array}{l}-- \\
\cdots \\
\cdots \\
--\end{array}$ & $\begin{array}{l}-- \\
\overline{-} \\
\overline{-} \\
\overline{-}\end{array}$ & $\begin{array}{l}4.5 \\
4.6 \\
4.6 \\
4.5 \\
4.8\end{array}$ & \begin{tabular}{l}
$-\cdots$ \\
$-\cdots$ \\
\hdashline-- \\
$-\cdots$
\end{tabular} & \begin{tabular}{l}
-- \\
\hdashline- \\
-
\end{tabular} \\
\hline $\begin{array}{l}11 \\
12 \\
13 \\
14 \\
15\end{array}$ & ב- & $\begin{array}{l}-\cdots \\
\overline{-} \\
\overline{-} \\
\overline{-}\end{array}$ & $\begin{array}{l}6.2 \\
5.7 \\
5.8 \\
5.3 \\
5.1\end{array}$ & $\begin{array}{l}6.4 \\
6.9 \\
7.3 \\
7.2 \\
7.0\end{array}$ & $\overline{-}$ & $\begin{array}{l}6.0 \\
5.1 \\
5.0 \\
5.3 \\
4.6\end{array}$ & $\begin{array}{l}4.8 \\
4.6 \\
5.0 \\
-.-\end{array}$ & $\begin{array}{l}\cdots \\
\cdots \\
\cdots \\
\cdots\end{array}$ & $\begin{array}{l}-- \\
-- \\
-- \\
--\end{array}$ & $\begin{array}{l}4.5 \\
5.0 \\
4.4 \\
4.3 \\
4.4\end{array}$ & $\begin{array}{l}-.- \\
-\cdots \\
-\cdots \\
-\cdots\end{array}$ & $\overline{-}$ \\
\hline $\begin{array}{l}16 \\
17 \\
18 \\
19 \\
20\end{array}$ & $\overline{-}$ & $\begin{array}{l}-- \\
\cdots- \\
-- \\
--\end{array}$ & $\begin{array}{l}5.2 \\
5.3 \\
5.1 \\
3.7 \\
-.-\end{array}$ & $\begin{array}{l}7.3 \\
6.9 \\
6.2 \\
6.6 \\
7.0\end{array}$ & \begin{tabular}{l}
..- \\
\hdashline 7.8 \\
7.8 \\
7.1
\end{tabular} & $\begin{array}{l}4.5 \\
4.2 \\
4.6 \\
4.4 \\
4.1\end{array}$ & $\begin{array}{l}-\cdots \\
\cdots-- \\
\cdots- \\
-\cdots\end{array}$ & $\begin{array}{l}\cdots \\
\cdots \\
\cdots \\
\cdots\end{array}$ & $\begin{array}{l}-.- \\
4.6 \\
4.8 \\
5.0\end{array}$ & \begin{tabular}{l}
4.4 \\
4.0 \\
3.7 \\
\hdashline.-
\end{tabular} & 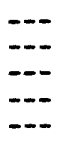 & $\begin{array}{l}-- \\
-- \\
-- \\
-- \\
\end{array}$ \\
\hline $\begin{array}{l}21 \\
22 \\
23 \\
24 \\
25\end{array}$ & $\overline{-}$ & $\begin{array}{l}\cdots- \\
\cdots \\
\cdots\end{array}$ & $\bar{m}$ & $\begin{array}{l}7.8 \\
8.4 \\
8.2 \\
7.9 \\
6.7\end{array}$ & $\begin{array}{l}7.0 \\
6.8 \\
6.3 \\
5.8 \\
5.6\end{array}$ & $\begin{array}{l}3.9 \\
4.1 \\
4.6 \\
4.6 \\
4.8\end{array}$ & $\bar{m}$ & $\bar{m}$ & $\begin{array}{l}4.8 \\
5.0 \\
5.1 \\
5.1 \\
4.7\end{array}$ & \begin{tabular}{l}
--- \\
\hdashline- \\
\hdashline- \\
\hdashline-
\end{tabular} & \begin{tabular}{l}
-- \\
$\overline{-}$ \\
\hdashline- \\
$-\cdots$
\end{tabular} & 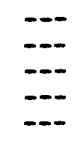 \\
\hline $\begin{array}{l}26 \\
27 \\
28 \\
29 \\
30 \\
31\end{array}$ & $\overline{-}$ & $\begin{array}{c}--- \\
-- \\
-- \\
-- \\
-- \\
\end{array}$ & $m$ & $\begin{array}{l}7.0 \\
7.7 \\
7.2 \\
6.8 \\
6.9 \\
6.2\end{array}$ & 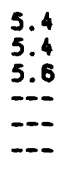 & $\begin{array}{l}4.8 \\
4.8 \\
4.7 \\
4.7 \\
4.7 \\
4.9\end{array}$ & $\overline{-}$ & 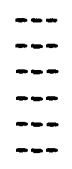 & $\begin{array}{r}4.6 \\
4.6 \\
4.6 \\
4.5 \\
4.6 \\
-. .\end{array}$ & \begin{tabular}{l}
--- \\
\hdashline- \\
\hdashline- \\
\hdashline- \\
$-\cdots$ \\
\end{tabular} & $m$ & $\begin{array}{c}\cdots \\
\cdots \\
\cdots \\
\cdots \\
\cdots\end{array}$ \\
\hline
\end{tabular}

$+$ 